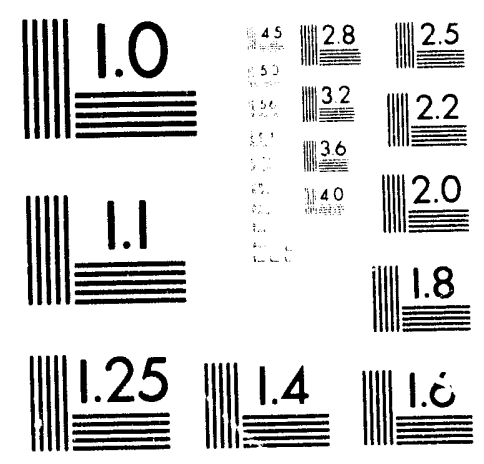



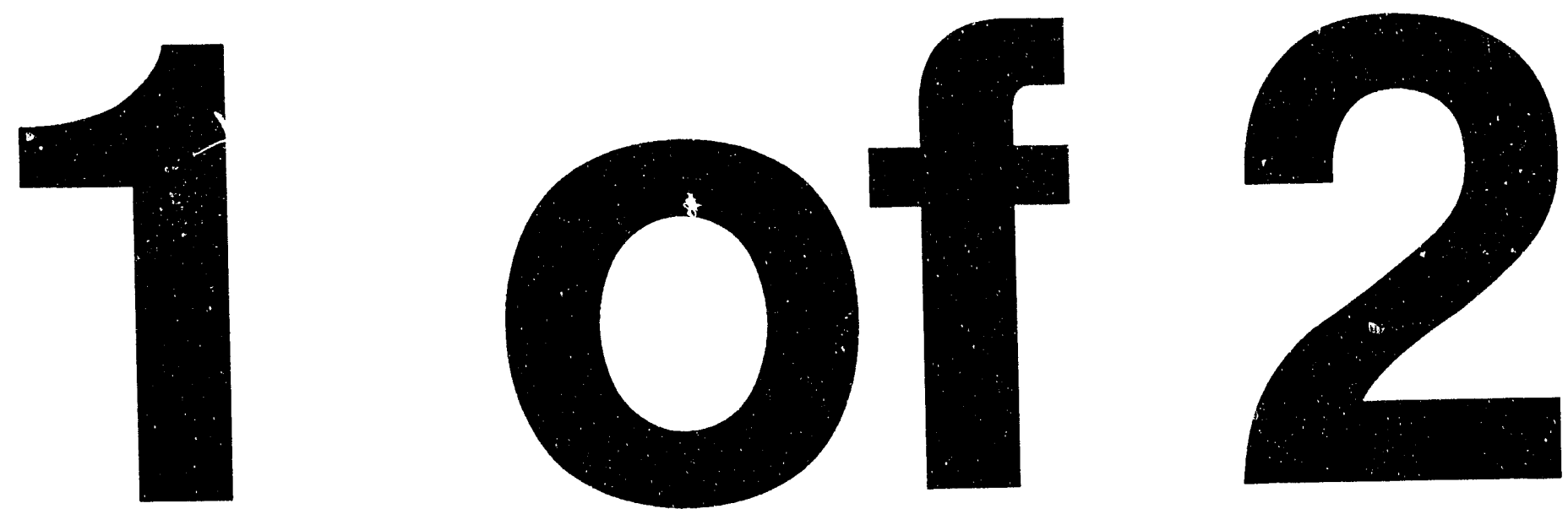
Tha submitted manuscript has been authored

Iby a contractor of the U.S. Government

under contract No. W.J1. TOGENG-J8.

Accordingly, the U.S. Governmitht retsins a

nonexclusive, royalty tree license to publish

or reproduce the publistied form of this

contribution, or allow others to do so, for

U. S. Government purposes.

\title{
Progress Report for the Scintillator Plate Calorimeter Subsystem
}

\author{
Argonne National Laboratory
}

Westinghouse Science and Technology center

BICRON Corporation

Introduction

Patent Cleared by

Patont Depertment, ANL

RELEAEE AUTHORIZED BY Techlinil Plidications Services

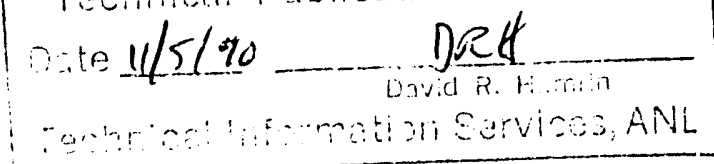

-

This report covers the work completed in FY90 by ANL staff and those of Westinghouse STC and BICRON Corporation under subcontract to ANL towards the design of a compensating calorimeter based on the use of scintillator plate as the sensitive medium. It is presented as five task sections dealing with respectively mechanical design; simulation studies; optical system design; electronics development; development of rad hard plastic scintillator and wavelength shifter and a summary. The work carried out by the University of Tennessee under a subcontract from ANL is reported separately. Finally, as principal institution with responsibility for the overall management of this subsystem effort, the summary here reports the conclusions resulting from the work of the collaboration and their impact on our proposed direction of effort in FY91. This proposal, for obvious reasons is given separately.

\section{MASTER}

$\because$

Work supported in part by the U.S. Department of Energy, Division of High Energy Physics, Contract W-31-109-ENG-38. 


\section{DISCLAIMER}

This report was prepared as an account of work sponsored by an agency of the United States Government. Neither the United States Government nor any agency thereof, nor any of their employees, makes any warranty, express or implied, or assumes any legal liability or responsibility for the accuracy, completeness, or usefulness of any information, apparatus, product, or process disclosed, or represents that its use would not infringe privately owned rights. Reference herein to any specific commercial product, process, or service by trade name, trademark, manufacturer, or otherwise does not necessarily constitute or imply its endorsement, recommendation, or favoring by the United States Government or any agency thereof. The views and opinions of authors expressed herein do not necessarily state or reflect those of the United States Government or any agency thereof. 


\section{SECTION IA.}

MECHANICAL DESIGN OF THE SCINTILLATINC PLATE CALORIMETER SUB́SÝSTEM

A. Buehring, N. Hill, J. Nasiatka, E. Petereic, L. Price, J. Proudfoot, H. Spinka, and D. Underwood

High Energy Physics Division Argonne National Laboratory, Argonne, Illinois 60439

and

M. Burke, D. Hackworth, T. Hordubay, D. Marshik,

$D$. Scherbarth, and $R$. Swensrud

Science and Technology Cencer

Westinghouse Electric Corporation, Pittsburgh, Pennsyivania 15235

\section{A) Introduction}

The calorimeter for an SSC general purpose detector should ideally have good energy resolution, fast time response, fine segmentation, and a high degree of hermeticity. For many SSC detector designs, the calorimeter is located outside a solenoidal magnet and tracking chambers, and weighs thousands of tons. The challenge is to develop a mechanical design that meets the goals stated above, is self-supporting with minimal structural elements (especially in the electromagnetic section), and is of modest cost.

The work for this subsystem is focussed on compensating scintillator plate calorimeters. It is expected that a sampling calorimeter with alternating "plates" of high-z absorber (lead, tungsten, depleted uranium) and plastic scintillator can be made compensating $(e / h=1.00)$ by the proper choice of thicknesses of the plates. This expectation is based on various experimental tests and on simulation studies. It is not clear that a steel-plastic 
scintillator calorimeter could achieve compensation. Thus, steel has not been considered as the absorber in these mechanical desigrs. Likíwise; the cost of pure tungsten as the absorber has removed it from consideration.

Two conceptual designs have been pursued for this subsystem. One is based on lead as the absorber, Eiber readout, and separate electromagnetic (EMC) and hadronic (HAC) sections. This allows replacement of scintillator and/or fibers if radiation damage effects become coo large. The barrel calorimeter support is taken to be from the "bottom" only. The other design is based on depleted uranium as the absorber, wavelength shifter (WLS) plate readout, nonseparable EMC and HAC sections, and a radial support of the barrel calorimeter from the muon iron.

A number of guidelines were developed for these two designs.

1) The calorimeter is separated into towers with three longitudinal sections each - one electromagnetic $\left(25 \mathrm{x}_{0}\right)$, and two hadronic (HACI, HACI I ).

2) The HACI and HACII sections are approximately projective, whereas the EMC section is rotated $8^{\circ}$ from purely radial. This reduces the effect of Cerenkov 1 ight in the fibers or Whs plates.

3) The total absorber thickness is a minimum of $9 \lambda$ in the barrel and $11 \lambda$ in the endcaps.

4) The solenoidal magnet is inside the calorimeters permitting a minimum inside diameter of the barrel to be $450 \mathrm{~cm}$.

5) The scintillator plates are approximately $11 \times 11 \mathrm{~cm}^{2}$ at the inside diameter of the varrel. The plates are parallel to the solenoidal axis in the barrel calorimeter and approximately perpendicuiar to the axis in the endcap calorimeter. These dimensions correspond roughly to $\Delta \eta=0.05$ and $\Delta \phi=0.05$. 
6) A $12^{\prime \prime}$ thick iron cylinder outside HACI is present for magnecic Elux return and phototube shielding in the barrel calorimeter, and $1.0 \mathrm{~m}$ in the endcap calorimeter. It may be used as a structural element.

7) Deflections from gravicy loads should be minimized ( $<0.7 \mathrm{~cm})$. In magnetizeable steel, such deflections could lead to sizeable forces on the solenoidal magnet coils. Also, the solenoidal magnet and cencral tracking detectors may be supported from the calorimeter with tight colerances. Finally, "cracks" in the calorimeter active volume may occur at the boundary between the barrel and endcap calorimeter sections.

8) Structural material inside the EMC should be minimized.

9) Scintillator plates, WLS plates, and fibers should not be compressed.

10) Calorimeter modules should be of transportabie size and weight.

11) The materials used in the design must withstand $>10$ Mrad over a ten year period.

12) Thermal effects should be considered. Temperature changes of 30$40^{\circ} \mathrm{C}$ may occur during shipping.

13) Fór the conceptual designs, a scintillator thickness of 2.5 mm and depleted uranium thickness of $3.3 \mathrm{~mm}$ was assumed to be compensating $(e / h=1.00 \pm 0.05)$. Similarly, $2.5 \mathrm{~mm}$ scintillator and $10.0 \mathrm{~mm}$ lead in the HAC sections was assumed to be compensating. However, $5.0 \mathrm{~mm}$ lead was assumed in the EMC to get improved energy resolution for electromagnetic showers. Simulation calculations proceeding in parallel with the mechanical design are expected to confirm that these thicknesses are approximately correct.

A zeroth order caiorimeter design is shown in Figs. 1.1 and 1.2 . These were used as starting points for the two conceptual designs. 


\section{B) Lead-Based Conceptual Design}

Westinghouse Science and Technology Center in Peñnsyluánia pèrtormed most

of the work on the conceptual design of a compensating calorimeter with lead

absorber. Initial calculations focussed on deformation of the full barrel

calorimeter under gravity loading and evaluation of stresses in the assembled

barrel. An estimate of stresses within a module during assembly was also

made. A study of lead materials (alloys and composite) was performed in parallel. On the combined basis of the stress calculations and materials study, a design concept for a lead calorimeter was developed. Furcher details on each of these zopics follow.

A module shape was firsc selected based on designs of other calorimeters and on the evaluation of various module shapes at Argonne. The barrel is assumed formed of 64 identical wedge-shaped modules, each module two towers wide. The endcap is assumed to be formed of 16 identical pie-shaped modules, each module up to eight towers wide.

\section{B.1 Barrel Calorimeter Deformation Calculations}

A finite element model was used to study deformations in the barrel calorimeter under gravity loading and to study stresses at various points within the barrel. Checks of this 2-dimensional model were performed using classical analytical calculations. The effects of the location of the bartel supports end of the coupling method between the modules were also studied with the model.

Various options for the form of the lead absorber were considered prior to the calculations of stresses and deformations. These options are illustrated in Fig. 1.3. For the barrel calorimeter, the three options were: 1) plates parallel to the axis of the bartel, 2) plates extending 


$$
-6-
$$

radially from the axis with slots punched for the scintillator tiles, and 3) cast modules with slots. The first option appeared to requíre reinéroment of the plates to accommodate compressive loading in the modules (see Sec. B.6). The second option would need binding ot the plates inco a module and machining of the surfaces. Alignment of the slots in the plates would be quite important. These extra steps and problems in the manufacturing of the modules for option 2 were expected to lead to higher costs than casting (option 3). For the second and third options, aligned or staggered scintillator slots were possible (see Fig. 1.4), but there was too little material between aligned slots to provide adequate strength in the EMC while maintaining uniformity of response to electromagnetic showers. As a result of the previous considerations, the cast lead module option with staggered slots was adopted for further study. We are indebted to J. Freeman and C. W. Foster (Fermilab) for their suggestions to consider some of these options. Similar Ideas had been evaluated earlier by the UAl collaboration at CERN, and perhaps other groups.

Analytical calculations were performed of the barrel calorimeter to estimate the magnitude of the deflections, and to provide a verification of the finite element midel. These calculations included a) lead in the HACI and HACII sections supported only at the bottom of the barrel, b) lead in the EMC section, c) the $12^{\prime \prime}$ steel cylinder containing a fluid with average density corresponding to the HAC lead, and d) a $6 \mathrm{~cm}$ thick steel cylinder containing a fluid with average density corresponding to the EMC lead. In all cases, the average density used for both the EMC and HAC calorimeter was that of an $80 \%$ lead, $20 \%$ plastic scintillator volumetric mixture. The estimated maximum deflections and the maximum tensile stress (at the bottom of the barrel) are summarized below: 

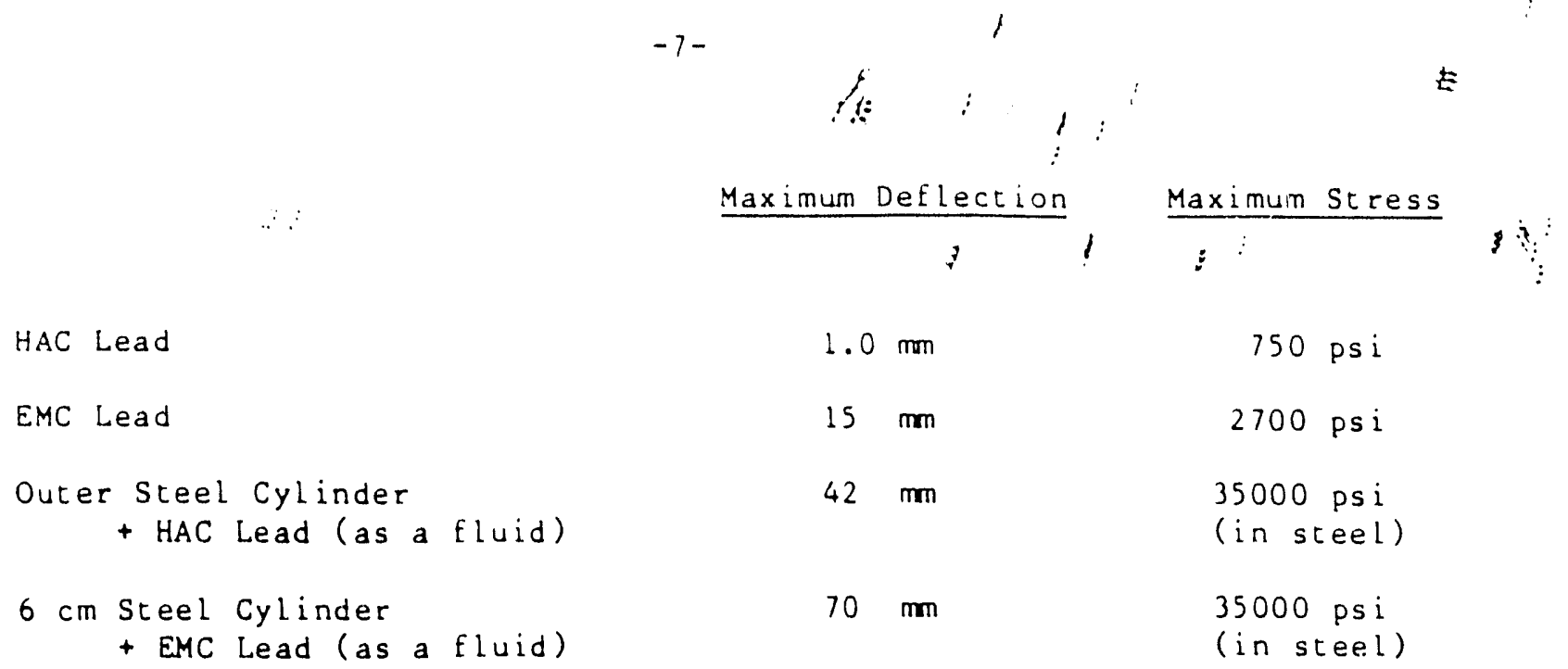

$\begin{array}{ll}1.0 \mathrm{~mm} & 750 \mathrm{psi} \\ 15 \mathrm{~mm} & 2700 \mathrm{psi} \\ 42 \mathrm{~mm} & 35000 \mathrm{psi} \\ & (\text { in steel) } \\ & 35000 \mathrm{psi} \\ 70 \mathrm{~mm} & (\text { in steel) }\end{array}$

In addition, estimates of the average compressional stresses at the horizontal midplane of the barrel calorineter gave 62 psi (confirmed by ANI. calculations). It was thought that such stresses might be small enough to employ pure lead in much of the calorimeter without significant creep.

Following the analytic calculations, a 2-dimensional finite element model of the barrel calorimeter was constructed (see Fig. 1.5). Half of the calorimeter was analyzed. A total of 128 elements were included - 32 modules, with each module separated into the EMC, HACI, HACII, and the steel outer cylinder. The calorimeter was assumed to be supported at the bottom and at one orher angle. In all cases considered, the steel outer ring elements were taken to be coupled' (for example, bolted at the inner and outer radius). The steel elasticity was taken to be isotropic, but the lead elasticity was anisotropic to allow for the slots in the lead to accept the scintillator tiles.

Several cases were considered; four are of primary interest for calculations of deflections. The maximum deflection, maximum stress, and method of connecting or "joining" of the elements between adjacent modules are summarized below. The elements could be coupled or bolted together; linked radially, such as with a dowel pin, but still allowed to move apart forming a gap; or "open", so that they could move apart, or deform and be at a different 


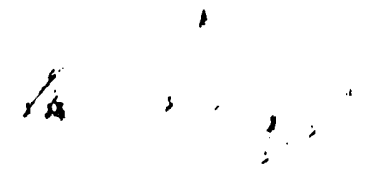

radius from the element in the adjacent module. The elements are not allowed to overlap, and the connections between elements within a mo'dule áre' assumed to be coupled or bolted. In all four cases, the barrel supports were taken to be located at $0^{\circ}$, or the bottom of the calorimeter, and at $\pm 28^{\circ}$.

$\begin{array}{ccccc}\text { Case } & \mathrm{C}_{2} & \mathrm{C}_{4} & & \mathrm{C}_{6} \\ \text { Inter-module Connection } & & & & \\ \text { EMC inner radius } & \text { Coupled } & \text { Open } & \text { Open } & \text { Open } \\ \text { EMC/HACI interface } & \text { Coupled } & \text { Linked } & \text { Open } & \text { Linked } \\ \text { HACI/HACII interface } & \text { Coupled } & \text { Open } & \text { Open } & \text { Linked } \\ \text { Maximum Deflection } & 2.5 \mathrm{~mm} & 12.6 \mathrm{mn} & 14.0 \mathrm{~mm} & 3.6 \mathrm{~mm} \\ \text { Peak Stress } & & 11,000 \mathrm{psi} & 10,000 \mathrm{psi} & 32,500 \mathrm{psi}\end{array}$

The peak stress includes a correction that increases the calculated maximum stress by a factor to account for the slots in the lead for the scintillator tiles. Local stresses at slot corners, etc. were not included in this global barrel calorimater model. The stress pattern in the lead for case CS is shown in Fig. 1.6 .

The conclusions drawn from these calculations are 1) the computed deflections are close to the desired limits. In particular, case C6 has a maximum deflection about half the limit suggested in guideline $\neq 7$, 2) All peak stresses are above the yield strength of pure lead or lead alloys. The need for lead composites is evident. Note that "room temperature" creep in the lead or lead alloys occurs at stresses above roughly 1000-3000 psi, an order of magnitude below the computed peak stresses in cases $\mathrm{C} 4, \mathrm{C} 5$, and $\mathrm{C} 6$. 
Finally, 3) the expected increase of peak stress with decrease in maximum

deflection is observed.

B.2) Deformation of the Barrel as a Function of the Location of the Supports

The same finite element model was used to evaluate the maximum deflection as a Eunction of the support angles. In these calculations, three supports were assumed - one at $0^{\circ}$ or the bottom of the barrel, and the others at $\pm \theta$. Curves showing the computed radial displacement at the inner bore of the barrel as a function of the azimuthal angle are given in Fig. 1.7. These curves correspond to different $\theta$ for case C5, and typically show the maximum displacement at the top of the barrel.

Figure 1.8 shows the maximum deflection as a function of the support angle $\theta$ for cases $C 2$ and $C 5$. The deflection is significantly reduced with increasing $\theta$. On the other hand, constraints from the location of the muon chambers and muon iron suggest possible limits to $\theta$ of about $30^{\circ}$ (see

Fig. 1.9). Therefore calculations performed for section $B .1$ above used $\theta=$ $28^{\circ}$ as a realistic support angle. The detailed design of the barrel support has not yet been performed, and therefore the size is not known.

\section{B.3 Barrel Calorimeter Module Design}

Both analytical and finite element calculations were performed for the design of a barrel module. Composites of lead and either nonmagnetic steel or cungsten have been considered in a "rebar-type" design. These calculations are continuing at present.

Two simple analytical calculations were performed for a module. One assumed the lead in the hadron section of the module was homogeneous and isotropic, with support from the $12^{\prime \prime}$ outer steel only. With the module horizontal, a peak tensile stress of $210 \mathrm{lb} / \mathrm{in}^{2}$ was computed. This estimate was 
expected to be subject to significant concentration factors resulting from the slots for the scintillator tiles. A second calculation was performed for a horizoncal module supported at the inner and outer radius only. This would occur tor example during shipping or handling for installation. The scintillator slots were approximated by considering the module to be plates at the inner and outer radius, with plates connecting them. The connecting plates were assumed to be lead with a chickness equal to the web thickness between towers $(\approx 4.8 \mathrm{~mm})$. The maximum stress was computed to be approximately 6000 lb/in ${ }^{2}$, significantly more than could be accommodated by pure lead or a lead alloy. In this case too, additional concentration factors might increase the estimated stress.

Once it was clear that a pure lead or lead alloy hadronic module could not withstand the stresses, a modified "rebar-type" module design was considered. This design has nonmagnetic stainless steel reinforcing plates at the EMC/HACI and at the HACI/HACII interfaces, in addition to the 12 "thick outer steel plate; see Fig. 1.10. Initially a cungsten or iron fiber mesh was also included. It was envisioned that the plates and fiber would be assembled first, and then the lead would be cast with slots for the scintillator tiles. The fiber mesh/lead composite would need to be optimized to achieve the required strength with a minimum of fiber mesh and of inhomogeneity in the module. This optimization was begun with a "wire-frame" finite element model of the module as shown in Fig. 1.11. It is a 3-dimensional model with three plates ( $1,14,27)$ and 24 wire elements.

An alternate modicie design is illustrated in Fig. 1.12 with thin nonmagnetic steel plates separating adjacent towers. The plates may have material removed as "cutouts". Work is proceeding to model the performance of such a module design. Input forces will be taken from the global calorimeter 
model described in section $B, 1$. Stresses in the plates will be evaluated to determin approximate dimensions. The response of the new module design to external forces will then be characterized by finite element calculations. The computed module response will be incorporated back into the full barrel model to reevaluate deflections and stesses. It is planned to verify the calculations by constructing small reinforced lead composite structures and measuring the response to various forces.

\subsection{Fiber Routing in the Hadron Calorimeter Barrel Modules}

The present conceptual design for the lead absorber and $f$ iber readout calorimeter assumes the $\mathrm{fiber}$ is embedded in a groove in the scintillator tile (see Fig. 1.13). The precise path is currently under study. Both ends of the fiber are taken out of the tile and routed to the outside of the barrel calorimeter. The fiber diameter is 0.7 or $1.0 \mathrm{~mm}$, and the minimum radius of curvature is approximately $2 \mathrm{~cm}$.

Three fiber routing schemes were considered: radial, axial, and projective. The radial routing scheme gives the shortest paths to exit from the bartel calorimeter for over $80 \%$ of the fibers. However, there are significant problems associated with the proper bundling of the fibers at the outside of the barrel for the projective towers if pure radial routing is used. Axial routing (along the beam direction) will presumably be used for the EMC section Of the barrel, and could conceivably also be employed for the HACI and HACII sections. There are also problems in this case with proper bundling of the fibers and with the longer average path length to reach the photodetectors. The average fiber path length for the projective routing is between the average radial and axial path lengths. Note that greater path lengths lead to 
larger dead spaces in the calu.imeter and higher light attenuation in the Eibers.

The projective routing scheme was adopted for the conceptual design as a compromise between Longer path length and easier assembly. It is presently envisioned that many scintillators for a single tower would be assembled in a "harness" with the fibers properly arranged and atcached together. All the scintillator tiles from the harness would be inserted into the slots in the Lead automatically to reduce assembly costs and time. This appears to require the fibers to exit from the scintillator tile tangentially and then to be bent perpendicular to the tile, as shown in Fig. 1.13. The fibers would be arranged in layers, in someshat the same fashion as wavelength shifter plates (Fig. 1.14). The cotal HACI and HACII tower readout would consist of 280 fibers from 140 scintillator tiles arranged in five layers for a total thickness of $\sim 3.5 \mathrm{~mm}$ at the outside of the batrel. For this design, at least $25 \%$ of the lead and steel surfaces would be available to be in contact with the material from the adjacent module. This permits loads to be transferred from module to module as assumed in Section B. 1 .

The fibers would exit from the barrel calorimeter through the $12^{\prime \prime}$ outer steel ring at the edges of the modules, and be arranged into two approximately circular bundles. The bundles would correspond to the HACI and HACII sections. The bundle diameters would be about $12.4 \mathrm{~mm}$ for $1.0 \mathrm{~mm}$ diameter fibers, and each bundle would be attached to a photodetector. The flexibility of the fibers would permit some or all of the photodetectors to be mounted at some distance from the towers. Thus it might be possible to minimize the distance between the muon chambers and the barrel calorimeter, while having access to all the photodetectors. 
A scheme was also developed to reduce the number of fibers, and hence the cost, by reading out more than one scintillator tile iin seríes (seje ig.

1.15). Some additional dead space results for more than two tiles in series, but this is compensated by the savings in space from the reduced number of Eibers. It is not clear thac the series readout scheme will be acceptable, since the time response of the calorimeter will be degraded.

\section{B.5 Endcap Conceptual Design}

Some work was begun on the design of the endcap calorimeter. For physics and cost reasons, it was atcempted to maintain the projective towers in the HACI and HACII sections. The total absorber thickness was taken to be $11 \lambda$, instead of $9 \lambda$ in the barrel, and the outer steel flux return was assumed to be $1.0 \mathrm{~m}$ chick (see Fig. 1.16).

There was a concern that the center of gravity of the endcap might be inside of the $1.0 \mathrm{~m}$ steel, requiring the endcap to be cantilevered. An approximate calculation showed that the combined center of gravity for the lead and steel was close to the interface of the HACII/outer steel for the largest angle cowers. Hence, the endcap could be supported in a stable fastion from the steel. For safety, the endcap could be installed with addirional counterweight outside the steel flux return. The barrel and endcap steel could be bolted together after installation, and the counterweight removerl if necessary.

Preparations were made for 3-dimensional finite element calculations of the endcap as shown in Fig. 1.17 and 1.18. The starting endcap concept included pie-shaped modules with purely projective hadron towers. It is anticipated that adjustments to this design will be made, since the scintillator tile size would become too small close to the beam. 
B.6 Lead Places in a ZEUS-Style Module Design

Some analytical calculations were performed assuning leb́d plates parallel to the barrel axis (as in Fig. 1.3) in a ZEus-Style design. These calculations complemented the results from the conceptual design for depleted uranium absorber in Sec. C. It was assumed that the lead plates were compressed with aluminum spacers. The scintillator tiles fit between the spacers .

One calculation considered a horizontal module supported only at the inner and outer barrel radius. The bending stress due to gravity at the module center of gravity was computed to be $177 \mathrm{lb} / \mathrm{in}^{2}$ and the maximum compressive stress at the lead absorbes/spacer interface was estimated to be approximately $11,600 \mathrm{lb} / \mathrm{in}^{2}$, including preload and bending stresses.

A second calculation considered a horizontal module cantilevered from the outer 12 " steel. In this case the bending stress at the module outer diameter was $210 \mathrm{lb} / \mathrm{in}^{2}$, and the maximum compressive stress at the lead absorber/spacer interface was approximately $16,900 \mathrm{lb} / \mathrm{in}^{2}$.

In both cases, the computed stresses are far higher than can be achieved with pure lead or lead alloy plates. It is thought that these stresses can be handled with a composite lead plate with steel reinforcement at high stress areas. This would lead to nonhomogeneous absorber plates.

\section{B.7 Cost Estimate for the Lead and Steel in the Barrel Calorimeter}

A very rough cost estimate for some of the materials in the barrel calorimeter was performed by an Argonne physicist. Using $3.8 \mathrm{~mm}$ slots, $5 \mathrm{~mm}$ lead in the EMC and $10 \mathrm{~mm}$ lead in the HAC sections of the bartel calorimeter, the total volume of lead is approximately $3.1 \times 10^{8} \mathrm{~cm}^{3}$, or the total weight is - 3900 cons. The general barrel shape is shown in Fig. 1.16, but the 


$$
-15-
$$

dimensions are slightly different. The reinforcing fiber mesh and plates were ignored in this estimate. Likewise, the cotal volume of the' outer' $1^{\prime \prime}$ steel, assuming it is in the form of a right circular cylinder, is approximately 1.2 $\times 10^{8} \mathrm{~cm}^{3}$ and the total weight is -1070 tons. At costs of $\$ 1.75$ and $\$ 0.50$ fer pound of lead and steel, respectively, the total costs of these materials will be $\$ 13.7 M$ and $\$ 1.1 M$. These values are summarized in the following cable.

$\begin{array}{clc}\text { Material } & \frac{\text { Cost }}{3900 \text { tons lead }} & \text { Total Cost } \\ 31.75 / 1 b . & \$ 13.7 M \\ 1070 \text { tons steel } & \$ 0.50 / 1 b . & \$ 1.1 M \\ & & \$ 14.8 M\end{array}$

The cotal volume of plastic scintillator was estimated to be $0.9 \times 10^{8} \mathrm{~cm}^{3}$ and the area $-3.6 \times 10^{8} \mathrm{~cm}^{2}$ for a $2.5 \mathrm{~mm}$ thickness. The total length of clear acrylic fiber was estimated to be $6210 \mathrm{~km}$ for the separable EMC section, but only $5050 \mathrm{~km}$ if the EMC is integral and all fibers are routed projectively. The total length of WhS fiber embedded in the plastic scintillator is roughly $500 \mathrm{~km}$. Costs for machining, shipping and handling, labor for assembly, fibers, scintillator, photodetectors, supports, electronics, inflation, contingency, etc. are not included in the value above.

\section{C) Depleted Uraniun - Based Conceptual Design}

Most of the calculations for the depleted uranium (DU) absorber, wavelength shifter (WLS) plate readout calorimeter were performed by personnel in the Argonne National Laboracory High Energy Physics Division. Some of the 
work was based on previous experience with the design and construction of the depleted uranium barrel caiorimeter for the ZEUS detetor at HERA:

The initial work concentrated on evaluating various module design alternatives for the barrel calorimeter. The EMC and HAC sections were assumed integral, with the HAC towers projective and the EMC sections tilted at $-8^{\circ}$ (see Fig. 1.2). The $8^{\circ}$ tilt led to an evaluation of different schemes to construct the EMC section and to difficulties with the mechanical design. As a result, "bulkheads" will probably be required on the module ends. A partial solution to the problem of cracks caused by the bulkheads was developed. Finite element calculations of a module and of the full barrel calorimeter were performed to evaluate stresses and deflections. Finally, a radial support scheme for the barrel calorimeter from the muon iron surrounding the barrel was evaluated. Details of these studies follow.

\section{C.1) Conceptual Design for Barrel Calorimeter Modules}

The barrel calorimeter with the short magnet coil was assumed to have 128 towers in $\phi$ and approximately 60 towers in $n(\Delta \phi=0.05, \Delta \eta=0.05)$. Nonprojective towers that could permit a simple and cheap "flat-washer" module design (see Fig. 1.19) were not considered because of shower localization and trigger problems. Similarly, a "projective-washer" module design as in Fig. 1.20 with one tower in $\eta$ and 128 towers in $\$$ was rejected. Such a module was too heavy ( $>80$ tons), too large to be easily transportable ( $10 \mathrm{~m}$ diameter), and would contain many small DU plates which would increase the total calorimeter cost. Although the absorber plates could be made perpendicular to a line through the incersection point, a varying amount of absorber would be required before the first scintillator tile. This material would be necessary to avoid a "sawtooth" inner surface to the EMC and to transfer stresses 
between modules. However, either solution (sawtooth surface or varying material) was expected to significantly degrade the dlectronagnet (energy resolution.

Therefore, modules two towers wide in $\Phi$ and many towers along $\eta$ were chosen for the conceptual design. In this case, the wLS plates are located on the outside surfaces of the modules, giving a one-sided readout of the scintillator tiles. The full barrel calorimeter with the short magnet coil was estimated to weigh $\geq 5000$ tons. Without longitudinal segmentation of the modules, their weight would be $\geq 78$ cons each. In order to keep the module weight at less than 30 tons, it was concluded that there should be three longitudinal $(n)$ segments. Note that there are manufacturing advantages to having only two, identical longitudinal barrel segments.

The boundaries to the longitudinal segments could be either projective (Fig. 1.21) or nonprojective (Fig. 1.22). A disadvantage of projective boundaries is the projective crack which would significantly affect the calorimeter response, especially if structural material was required on the module ends. The disadvantage of nonprojective boundaries is related to the readout. As seen in Fig. 1.22, the segment boundaries would intersect a number of towers. Additional whs plates would be needed to read out the partial EMC or HAC sections at the boundaries, resulting in more dead space in the calorimeter. Such problems would be reduced with fiber readout. However, additional photodetectors and electronics channels would be required for either readout, contributing added cost and trigger complexity.

Projective boundaries to the Longitudinal barrel segments were chosen for the conceptual design. To minimize the impact of the projective boundary cracks, the towers are made slightly nonprojective in $n$. In the present design, the seven hadron towers in $n$ centered on $n=0$ are made to project 
perpendicular to the beamline (Like the flat-washer solution). The remaining hadron towers project to a point on the beamline apprbximately $\pm \dot{3} 9 \mathrm{~cm}$ away trom the intersection point. Similarly, the segment boundaries project to these points. A straight line from the true intersection point crosses -1.25 - 2.0 towers in the barrel calorimeter for essentially all towers with $n \neq 0$ in the scheme described above (see Fig. 1.21).

\section{C.2 Calculations of Stresses and Deflections in a Barrel Module}

For the analysis of a barrel module, a series of 2-dimensional finite element calculations were performed in addition to analytic computations of deflections. It was initially assumed that the final design would be similar to the zEUS module design. The DU plates are assumed to be under compression with aluminum spacers between the plates. (For some early calculations, the scintillator tiles were assumed to be compressed between the DU plates.) The compression is provided by the combination of an inner aluminum or steel plate, a center steel tension plate to provide added stability, an intermediate steel plate at the HACI/HACI interface, thin steel skins, and a thick outer steel plate at the outer diameter of the barrel calorimeter. The conceptual module design is shown in Fig. 1.23 .

Several classical analytic calculations were performed for the barrel module. For the first, the module was assumed to be vertical, such as during assembly and transportation. In this case, the three steel plates act as cantilevered beams. Maximum deflections for various thicknesses and materials for the inner plate are tabulated below. It is assumed that the entire load from the DU plates is uniformly distributed across the inner plate. In all cases, $1.6 \mathrm{~mm}$ thick steel skins were assumed. 


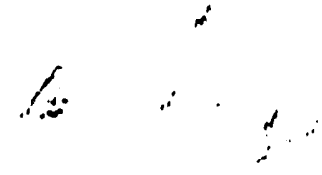

\begin{tabular}{|c|c|c|c|c|c|}
\hline \multirow[b]{2}{*}{$\begin{array}{c}\text { Inner Plate } \\
\text { Thickness }\end{array}$} & \multicolumn{2}{|c|}{ Steel } & \multicolumn{3}{|c|}{ Alumi num } \\
\hline & $\begin{array}{l}\text { Without } \\
\text { Skins } \\
\end{array}$ & $\begin{array}{l}\text { With } \\
\text { Skins } \\
\end{array}$ & $\begin{array}{l}\text { Writ hout } \\
\text { Skins } \\
\end{array}$ & $!$ & $\begin{array}{l}\dot{w} \text { with } \\
\text { Skins }\end{array}$ \\
\hline $12.7 \mathrm{~mm}$ & $0.4 \mathrm{~mm}$ & $<0.1 \mathrm{~mm}$ & $1.2 \mathrm{~mm}$ & & $<0.1 \mathrm{~mm}$ \\
\hline $19.0 \mathrm{~mm}$ & $0.1 \mathrm{~mm}$ & $<0.1 \mathrm{~mm}$ & $0.3 \mathrm{~mm}$ & & $<0.1 \mathrm{~mm}$ \\
\hline
\end{tabular}

For the second analytic calculation, the module was assumed to be horizontal. It was shown that an uncensioned steel plate could not support the weight of a module unless it was very thick. When the plate was prestressed at the supporting edges, the deflections become manageable ior thin plates. The calculations assumed the plate had the dimensions of the central tension plate, was supported only at the two ends and had the module weight uniformly distributed along the plate. For a $6.35 \mathrm{~mm}$ thick steel center plate tensioned to 40,000 psi, the maximum deflection was estimated to be $16 \mathrm{~mm}$. With the addition of two $1.6 \mathrm{~mm}$ thick steel skins tensioned to 20,000 psi each, the maximum deflection was very small. However, without adequate precautions the skins could cause pressure on the WLS plates. Thus it was hoped that these skins would not be required except for purposes of optical isolation from the adjacent inodules.

A third analytical calculation treated the module as a solid block of material supported from the ends. With mechanical properties of DU, the resulting total deflection was $<0.1 \mathrm{~mm}$. This provides a lower limit to the expected deflection.

Several finite element model calculations of a barrel module were also performed. One model congisted of beam elements with dimensions as shown in Fig. 1.24. The module was vertical and suspended at the top and center of the T-beam. The inner, intermediate, and outer steel plates were connected with non-compressible elements to share the load. The weight of the module was 
applied uniformly across the bortom or inner plate. The maximum deflection was calculated to be $0.04 \mathrm{~mm}$ and the maximum bending istress was 500 psi.

This same module model was assumed for a horizontal module. The T-beam was fixed in place at the outer ring, and supported at either che inner plate or both the inner and intermediate plates (Fig. 1.25). The center plate was uniformly loaded with a pressure equal to the weight of the module. The deflections were calculated to be 0.9 and $0.3 \mathrm{~mm}$ for the case of two and three supporting plates, respectively, which are quite manageable.

Another simple 2-dimensional finite element model of a barrel calorimeter was also used to compute stresses and deflections. No intermediate plate was assumed, and the inner plate was taken to be aluminum. Thus the deflections were higher than for one of the previous module finite element calculations. The module was assumed to be vertical with the inner plate at the bottom to maximize the stresses. The center plate was taken to be $6.35 \mathrm{~mm}$ thick, the outer plate $38.1 \mathrm{~mm}=1.5^{\prime \prime}$ thick, and the skins each $1.6 \mathrm{~mm}$ thick. The model consisted of 53 plate elements and 46 nodes, and it assumed pressure on the inner and outer plates to produce a total tension of $31001 \mathrm{~b}$. in the center plate. The module weight was assumed to be uniformly distributed across the inner aluminum plate, adding to the pressure from the center plate tensioning. The results are tabulated below. Note that additional concentration factors, in areas such as near the spacers between the DU plates, might increase the estimated stresses.

Case

$19.0 \mathrm{~mm}$ inner plate, with skins

$12.7 \mathrm{~mm}$ inner place, with skins

$19.0 \mathrm{~mm}$ inner plate, without skins

$12.7 \mathrm{~mm}$ inner plate, without skins
Inner Plate Maximum Deflection

$$
\begin{aligned}
& 0.28 \mathrm{~mm} \\
& 0.48 \mathrm{~mm} \\
& 1.22 \mathrm{~mm} \\
& 4.14 \mathrm{~mm}
\end{aligned}
$$

Inner Plate Maximum Stress

$$
\begin{aligned}
& 16,400 \text { psi } \\
& 29,600 \text { psi } \\
& 39,200 \text { psi } \\
& 88,500 \text { psi }
\end{aligned}
$$


The conclusions drawn from these calculations are: 1) the skins are essential to reduce deflections and stresses to acceptable l'evel $g^{\circ}$ when using chin aluminum inner plates. 2) an inner plate chickness $>13 \mathrm{~mm}$ aluminum is required. Note that the inner plate is actually located at the EMC/HAC I interface, since most of the module weight is in the HAC sections. The EMC section could then be attached to the rest of the module with significantly Less material inside of the first EMC scintillator tile. 3) An inner steel plate of thickness - 13-19 mm may be adequate without tensioned skins.

\section{3 Finite Element Analysis of the Barrel Calorimeter}

Following the analysis of a single barrel module described in the previous section, a 2-dimensional model of the fully assembled barrel calorimeter with DU absorber was evaluated for stresses and deflections. This model is similar to the one developed for the lead conceptual design (see Section B.1). The barrel consists of a $19 \mathrm{~mm}$ thick inner steel ring, a solid stack of compressed DU plates with scintillator tiles forming HACI, a $16 \mathrm{~mm}$ thick steel intermediate ring, another stack of DU and scintillator for HACII, and a 12" thick steel outer ring (see Fig. 1.26). The DU absorber/scintillator stacks were assumed to be solid anisotropic bodies. The three steel rings could be used to transfer loads to adjacent modules. A gap was required between DU/gcintillator stacks to avoid crushing the WLS plate.

The single module was represented by a total of $4 \times 13$ plate elements. The inner and outer rings consisted of $4 \times 2$ elements, the intermediate ring of $4 \times 1$ elements, and the HACI and HACII of $4 \times 4$ elements each. The module was rotated around the beam axis to form the barrel as show in Fig. 1.27, with each ring connected or bolted together, and with gaps at HACI and HACII as shown in Fig. 1.26 . 
Several cases corresponding to different supports were evaluaced. These included a single support at the bot tom of the barrel", a "saddle" or "cradle" extending $\pm 30^{\circ}$ from the bottom, and saddles at both the top and bottom extending $\pm 22.5^{\circ}$ and $\pm 28.1^{\circ}$. The results are tabulated below. Additional concentration factors may apply near the aluminum spacers.

$\begin{array}{ccc}\text { Case } & \begin{array}{c}\text { Maximum } \\ \text { Displacement }\end{array} & \begin{array}{c}\text { Maximum } \\ \text { Stress }\end{array} \\ \text { Support at Bottom Only } & 0.76 \mathrm{~mm} & 8700 \mathrm{psi} \\ \pm 30^{\circ} \text { Saddle at Bottom } & 0.41 \mathrm{~mm} & 7000 \mathrm{psi} \\ \pm 22.5^{\circ} \text { Saddles at Top and Bottom } & 0.08 \mathrm{~mm} & 1100 \mathrm{psi} \\ \pm 28.1^{\circ} \text { Saddles at Top and Botcom } & 0.05 \mathrm{~mm} & 900 \mathrm{psi}\end{array}$

Deflections with the model with $\pm 22.5^{\circ}$ saddles on top and botrom are shown in Eig. 1.28. The support case with $\pm 22.5^{\circ}$ sadjie(s) is convenient because the muon iron outside the barrel is expected to be oclagonal, which naturally gives rise to a $\pm 22.5^{\circ}$ angle (see Fig. 1.9). The supports could be in the form of discrete rails extending across the length of the barrel.

Finally, with changes in the mecinanical constants for the materials and in the dimensions, this model is also applicable to a cast lead barrel calorimeter. Cross checks with the Westinghouse model in Section B.l gave reasonable agreement of stresses, for example. Another check of the finite element model was also performed for a steel ring with inside diameter $5.08 \mathrm{~m}$, outside diameter $10.16 \mathrm{~m}$, and thickness $10 \mathrm{~cm}$. The ring was supported at the bottom only, and the load was approximately the weight of the corresponding volume in the barrel calorimeter. Both a classical analytical calculation and the finite element model results yielded a maximum deflection at the top of the ring of $0.28 \mathrm{~mm}$. 


\section{C.4 Radial Supports for the Barrel Calorimeter}

Calculations for supporting the bartel calorimeter radílly to the

surrounding muon iron were performed. Assuming 16 supports equally spaced around the barrel (see Fig. 1.29), and 30 supports along the barrel axis, the estimated loads in each support ranged up to 100,000 1b. at the barrel top. If the radial supports were in the form of turnbuckles, and a safety factor of 2.0 is assumed, the turnbuckle diameter would be $7 \mathrm{~cm}$. The difficulty is accessing the turnbuckles for adjustment and the large computed size eliminated this method as a viable scheme for the full support of the barrel calorimeter. However, there may be need for such radial support to complement other support methods.

\section{C.5) The EMC Section of the Barrel Calorimeter}

In order tu eliminate radially projective cracks (cracks occurring at constant $\phi)$, the zEUS barrel calorimeter has the modules rotated slightly as seen in Fig. 1.30. Relatively thick endplates or bulkheads, approximo.ely 6 $\mathrm{cm}$ of aluminum and steel, are used to help support the loads on the modules. The inner surface of the EMC is sawtoothed. In contrast, the SSC subsystem conceptual designs have HAC towers that are projective in $\Phi$, and the EMC sections are rotated $\sim 8^{\circ}$. However, the EMC scincillator tiles are not tilted in order to avoid the sawtooth problem. (The $8^{\circ}$ rotation was shown to be approximately optimum in zEUS studies.)

Various options to construct the EMC barrel calorimeter from DU plates were considered. Radial DU plates with punched slots, as in Fig. 1.3, were rejected for several reasons. For compensation, the DU thickness was assumed to be $3.3 \mathrm{~mm}$ and the scintillator thickness $2.5 \mathrm{~mm}$. However, allowing for the optical mask, wrapping and cushioning around the scintillator tile, and for 
mechanical tolerances, the slot width was estimated to be $\sim 4.2 \mathrm{~mm}$. Thus the slot is wider than the DU "plate". As a consequence," staggering "̈he slots does not significantly improve the problem of too little material between slots compared to aligned slots (see Fig. 1.31). Compared to the case with plates parallel to the barrel axis, edditional costs result from A) the much larger amount of DU that must be processed into sheets, B) punching of the precision slots, and C) machining the finished module to give smooth sides.

A number of options with plates parallel to the barrel axis were also considered. DU plates with staggered overlapping edges (Fig. 1.32) was rejected as an option because maintaining the correct overlap and keeping plates in position during assembly would be difficult. Costs associated with the larger number of DU plates would also be higher. Another possible option was to compress the scintillator between long DU plates, which violates guideline $\$ 9$. Tests of the effects of compressive loading on scintillator are being prepared, but no results have been obtained so far; see Section C.6. The option chosen for the conceptual design was similar to that in ZEUS - long DU plates, parallel to the barrel axis, separated by aluminum spacers. Considerable practical experience with this option exiscs and the .. problems are well known and understood.

The $\sim 8^{\circ}$ tilt to the EMC section introduces a number of problems, primarily related to the compression of the plates and to the width of the WLS plate. In one design under consideration, steel skins and the center plate are used to compress the full module as shown in Fig. 1.33. On the sides of the module, spacers and bolts atcach the skins to the plate between the EMC and HACI sections, causing a rediction in the width of the wLS plates from the EMC. The EMC plates are not compressed in a direction normal to their surfaces, rather at $\sim 8^{\circ}$ to the normal. This causes a force parallel to the 


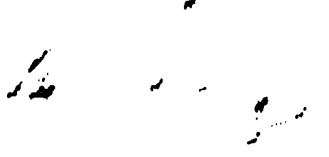

plate surface, in addition to loads from plate weight and from adjacent

modules. These loads are opposed by frictional f. rces and by bulkheads on the module ends and perhaps within the EMC section. In a second design, thin straps that follow the tower boundaries and pass through slots in thu DU plates (Eig. 1.34) provide compression in the EMC section. Compression for the HAC section is provided by the center plate and by skins bolted to the plate between the EMC and HACI sections, also reducing the width of the EMC WLS plates. In both designs, the center tensiun plate extends through the HAC sections only, to minimize structural material in the EMC, and the EMC WLS plates have an $8^{\circ}$ bend.

The number of strans required to adequately support the EMC depends on the weight of the EMC and the coefficient of friction ( $\mu$ ) between the DU plates and the aluminum spacers. The coefficient of friction was measured in ZEUS to be $\mu \cong 0.2$, but a value of $\mu=0.1$ is used in calculations for safety. The present design assumes two steel straps per tower, each tensioned to $12,000 \mathrm{ps} 1$. The sr.rap cross sectional area would be $0.5 \mathrm{~mm} \times 25.4 \mathrm{~mm}$. The minimum thickness and number of bulkheads and the minimum thickness of the plate at the front of the EMC have not yet been determined.

\section{6 Compression Tests on Scintillaror}

In some mechanicat designs with a stack of absorber plates and scintillator tiles, there are advantages in compressing the stack without spacers between the absorber plates. For example, local stresses in the plates are decreased. The fiducial volume is increased when spacers are eliminated. There is also the possibility in some sead absorber calorimeter designs that local stresge: may cause the lead to creep, resulting in compression of the scintillator tiles. 
As a result of these cunsiderations, a fixture has been designed and is under construction at Argonne that will al Low measurements off the "effects of compression on the light output from plastic scintillator. It is planned to have several short stacks of scintillator and absorber. One stack would serve as a control. Another would be used for rapid compression or slow compression studies, and another for compression of irradiated scintillator tiles, etc. The scintillators will be viewed with photomultipliers and the pulse height recorded in Argonne designed ADC's. Calibrations will be performed with cosmic rays and/or a laser. It is expected that the compression tests will begin soon.

\section{C.7) Costs of Depleted Uranium, Steel, and Scintillator for the Barrel}

\section{Calorimeter}

Volumes of DU, steel, WLS plates, and scintillator were computed using similar assumptions as for the lead design. Volumes were $1.2 \times 10^{8} \mathrm{~cm}^{3} \mathrm{steel}$ and $1.9 \times 10^{8} \mathrm{~cm}^{3}$ DU. The costs are summarized in the following table.

$\begin{array}{ccc}\frac{\text { Material }}{n} & \text { Cost } & \text { Total Cost } \\ 4000 \text { tons DU } & \$ 6.00 / 1 \mathrm{~b} . & \$ 48.0 \mathrm{M} \\ 1050 \text { tons steel } & \$ 0.50 / 1 \mathrm{~b} . & \$ 1.1 \mathrm{M} \\ & & \$ 59.1 \mathrm{M}\end{array}$

The estimated areas for scintillator were $5.7 \times 10^{8} \mathrm{~cm}^{2}$ and for whs plates 7.1 $x 10^{7} \mathrm{~cm}^{2}$. Costs for machining, shipping and handling, labor for assembly, WLS plates, scintillator, photodetectors, supports, electronics, inflation, contingency, etc. are not included in the value above. 
D) Summary

A summary of the conclusions from mechanical design stüdies óf lead and depleted uranium absorber calorimeters follows. Several unanswered questions remain, and some of these will be addressed in future work.

1) Pure lead or lead alloys will not work as the absorber for the hadron section of the barrel calorimeter. Stresses are too high for either lead plates or cast lead modules. Lead composites, reinforced with steel, tungsten or some other material will be required.

Consequently, the absorber will be inhomogeneous (see Figs. 1.10 and 1.12). It is not yet clear whether composites will be necessary in the EMC.

2) For the hadron calorimeter, casting lead in a steel frame with steel reinforcing and with staggered slots appears most economical. The slots can be cast around teflon or plastic forms. It has not yet been established that the slot thicknesses or the slot spacings can be made sufficiently uniform so they do not limit the calorimeter energy resolution. Likewise, the impact of staggered slots on the EMC performance must be studied.

3) A" calorimeter design with DU absorber and WLS plate readout, similar to ZEUS, seems to work acceptably. However, the DU processing costs and the poor fiducial volume resulting from the WLS plates are primary problems of this design compared to the design with fiber readout and lead absorber.

4) A variety of schemes to cake advantage of the small size and good flexibility of the fibers exist. The lead absorber design shows reduced cracks between modules with the use of fibers. The photodetector location can be more accessible with fibers than with 


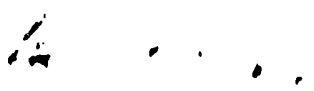

WLS plate readout. A scheme for reading multiple scintillator tiles in series has also been developed. However, it has not yet heen established whether the routing method in Figs. 1.13 and 1.14 will permic a sufficiencly uniform response in the scintillator tiles, or whether the fibers can withstand the radiation doses almost everywhere in the calorimeter.

5) Having the EMC modules separate from the HAC modules will probably reduce the size of cracks in the EMC, while also minimizing material inside the first EMC scintillator layer. The EMC modules do not necessarily need to be removable. Fiber readout would be required.

6) The hadron section of the barrel calorimeter modules are similar for the two conceptual designs studied. Both designs have evolved toward a module that is two towers wide in and many towers long in $n$ (Eigs. 1.10,1.12,1.23). Both contain intermediate steel reinforcing plates and both utilize the outer $12^{\prime \prime}$ steel for structural strength as well as magnetic flux return and shielding for the photodetectors.

7) The barrel calorimeter will need to be in two or more sections Longitudinally for the modules to be easily transportable. For three longitudinal sections, a scheme exists to reduce the effects of cracks and structural material at the ends of the modules. This involves hadron towers that are quasiprojective in $n$ (see section C.1).

8) There are problems for supporting the barrel calorimeter. Pure radial supports (Section C.4) take up too much space and will be difficult to access and adjust within the muon iron. Cradle or 
saddle supports from the botcom restrict the muon coverage asymerically.

9) The endcap towers probably can be projective. The endcap may not need to be cantilevered from the support on the bottom of the atlached $1.0 \mathrm{~m}$ muon iron and flux return. However, more work is needed to develop a good endcap conceptual design.

10) Although very rough cost estimates for some parts of the two calorimeter conceptual designs exist (Sections B.7 and C.7), much more detailed costing is required.

The subsystem collaboration met on 20-22 August 1990 to review progress on the mechanical design, material studies, simulation, etc. Based on the work presented, it was concluded that the lead absorber design should be pursued further. The primary motivations were cost and time response of lead compared to DU as the absorber from simulation calculations. Fiber readout was adopted because of its many advantages compared to WLS plates. However, it was also concluded that there are many similarities in the two conceptual designs. Thus wLS plate readout is considered a "backup option" should a serious problem appear with fiber readout. Similarly, DU or steel may be considered in case the lead absorber design encounters serious problems. 


\section{FIGUEE CAPT IONS}

Eigure 1.1 Starting design for the calorimeter showing projective :owers and a "short" magnet coil inside the barrel calorimeter.

Figure 1.2 Cross sectional view through the barrel calorimeter. The hadronic towers are projective, while the electromagnetic cowers are tilted at approximately $8^{\circ}$.

Eigure 1.3 Options considered for the form of the lead absorber. Lower right-plates parallel to the barrel axis. Middle-radial plates with punched slots. Top-cast module with slots.

Eigure 1.4 Staggered (left) or aligned (right) slots for the scintillator tiles in a cast module or a module with radial plates.

Figure 1.5 Description of the 2-dimensional finite element model for the barrel calorimeter.

Figure 1.6 Stress pattern for case $C 5$ in the barrel calorimeter lead from a two-dimensional finite element model.

Figure 1.7 Curves showing computed radial displacement as a function of angle $\left(0^{\circ}=\right.$ bottom, $180^{\circ}=$ top $)$ in the barrel calorimeter. The curves are labeled with the angles of the supports. All curves correspond to the finite element model $\mathrm{CS}$ described in the text.

Figure 1.8 Maximum deflection in the hadron barrel calorimeter as a function of the support angle. Results for the finite element models $C 2$ and $C 5$, described in the text, are shown.

Figure 1.9 Possible support locations considered for the barrel calorimeter. 
Figure 1.10 Conceptual design of a lead hadronic calorimeter barrel module showing the three iron or steel plate's and the turigsten or iron fiber mesh reinforcing.

Figure 1.11 Schematic diagram showing the 3-dimensional "wire-frame" finite element model of a lead hadronic calorimeter barrel module.

Figure 1.12 Conceptual design of a lead hadronic calorimeter barrel module showing the outer $12^{\prime \prime}$ iron plate, a $16 \mathrm{~mm}$ steel plate between the HACI and HACII sections and a $38 \mathrm{~mm}$ aluminum plate between the EMC and HACI sections. Thin steel plates are present between towers for reinforcing.

Eigure 1.1.3 Schematic diagram of fiber routing in grooves in a scintillator tile. Two possible paths in the tile are shown. The optimum routing path is under study. Figure 1.14 Schematic diagram of $f$ iber routing from a number of scintillator tiles into a readout bundle.

Figure 1.15 Possible method of fiber readout arrangement for two or more scintillator tiles in series. For the special case of two tiles, some dead space can be saved by having the fiber on the bottom surface of the upper tile.

Figure 1.16 Dimensions for the barrel and endcap calorimeters with the short magnet coil option. The outer steel flux return is at least $12^{\prime \prime}$ thick for the barrel and $1.0 \mathrm{~m}$ thick for the endcap. The approximate center of gravicy of the endcap calorimeter is indicated.

Figures 1.17-18 Conceptual design of the endcap calorimeter. 
Figure 1.19 Conceptual design of a calorimeter with "flat washer" modules (nonprojective cowers).

Figure 1.20 Conceptual design of a calorimeter with "projective washer" barrel modules. The insert shows the inner surface of the modules. The material will be of varying thickness across the face of the tower in order to transfer loads to adjacent modules and to prevent a sawtooth inner EMC surface.

Figure 1.21 Conceptual design of quasiprojective hadron towers and quasiprojective cracks at the ends of modules. The true intersection point is along the beamline at the far left. The seven towers centered on $n=0$ have boundaries in planes perpendicular to the beamline. The boundaries of all other towers project to an offset point along the beamline as shown by the dashed lines.

Figure 1.22 Conceptual design of a barrel calorimeter with nonprojective boundaries between sections. A number of towers are split by the boundary, requiring additional space for whs plates or fibers and additional readout channels.

Figure $1.23^{\circ}$ A barrel calorimeter module cross sectional view with DU absorber and WLS plate readout. The center steel plate, inner, intermediate and outer steel plates, and the thin skins are shown. The EMC section is tilted at $8^{\circ}$, while the HACI and HACII sections are projective in $\phi$.

Figure 1.24 Finite element model of a DU absorber barrel calorimeter module used to study stresses and deflections in a vertical module. The model consists of beam elements corresponding to a $38 \mathrm{~mm}$ outer plate, a $16 \mathrm{~mm}$ intermediate plate, a $19 \mathrm{~mm}$ inner 
Figure 1.25 Finite element calculation of a horizontal barrel calorimeter module. The same plate dimensions are used as in the previous figure. The module is fixed at the right (outer plate), is tensioned and supported at the inner plate, and is supported at the intermediate plate. The module weight is uniformly distributed across the center plate.

Figure 1.26 Finite element model of the full barrel calorimeter showing thrie adjacent modules. Only the HACI and HACII sections are included.

Figure 1.27 Finite element model of the full barrel calorimeter with DU absorber, and whS plate readout. The support shown is from the bottom only. Other cases were also evaluated.

Figure 1.28 Deflection pattern in the barrel calorimeter with DU absorber from a two-dimensional finite element model. The support is from the top and bottom with a $\pm 22.5^{\circ}$ saddle.

Eigure 1.29 Cross sectional view through the barrel calorimeter showing the locations of the radial supports to the muon iron.

Eigure 1.30 Cross sectional view through the ZEUS barrel calorimeter, illustrating the sawtooth inner surface caused by the slightly rotated modules. 
Figure 1.31 Staggered slots for DU absorber and scintillator tiles with approximately correct relative dimenstions. "The stots are Larger than the $2.5 \mathrm{~mm}$ scintillator to allow for the mask, wrapping, clearance and tolerances.

Eigure 1.32 View of $\mathrm{flat}$ DU plates with small overlap.

Eigure 1.33 Detailed view of the tilted EMC section and inner HACI section of a barrel calorimeter module. Thin steel skins are used to compress the EMC DU absorber plates, leading to a force tangential to the plates.

Figure 1.34 Conceptual design of thin steel straps to compress the EMC section of a barrel calorimeter module. The straps follow tower boundaries. 


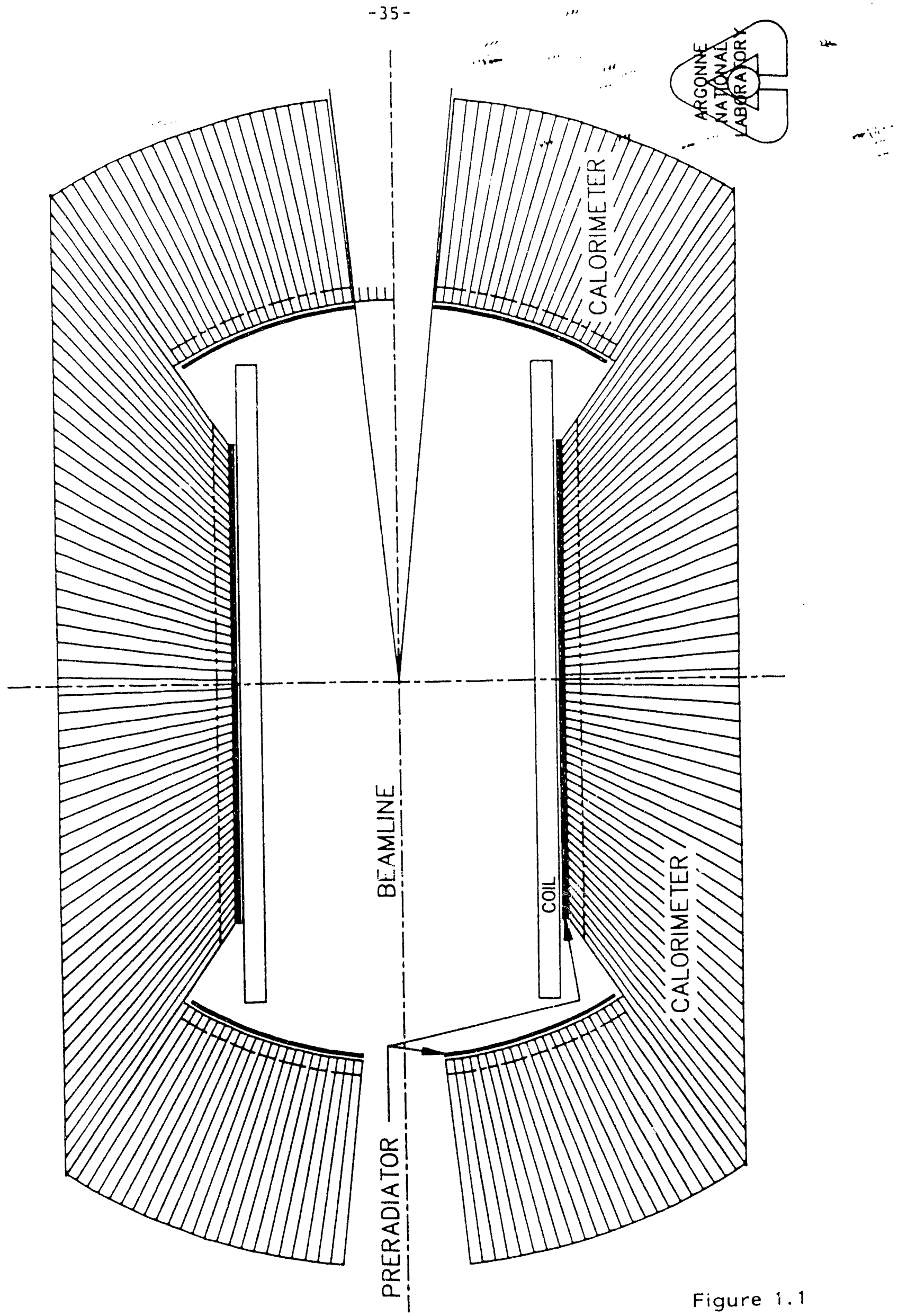




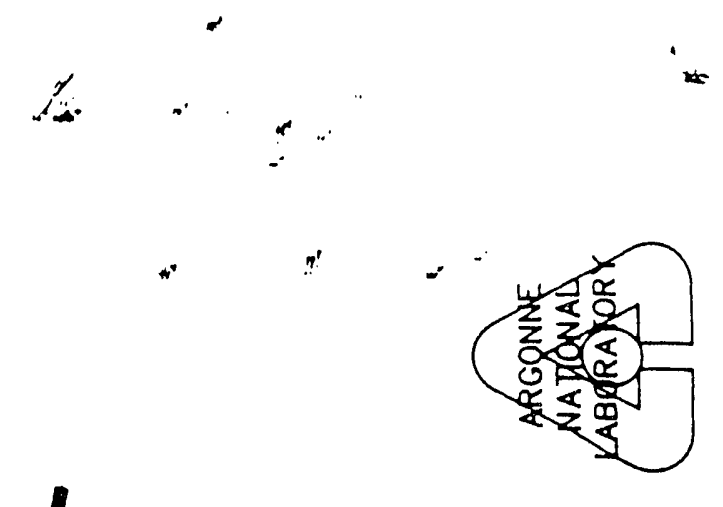

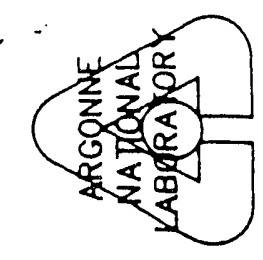

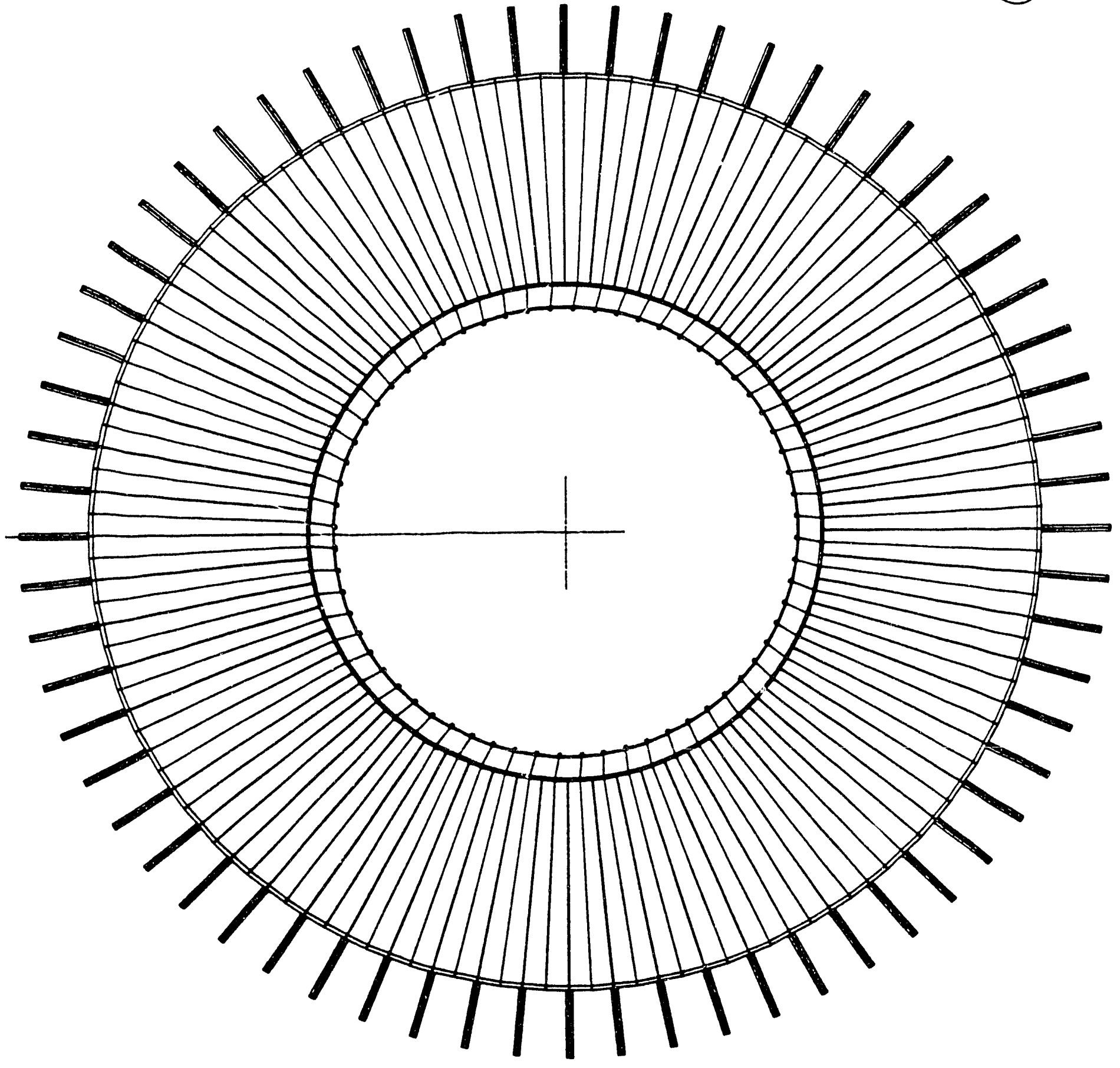

Figure 1.2 


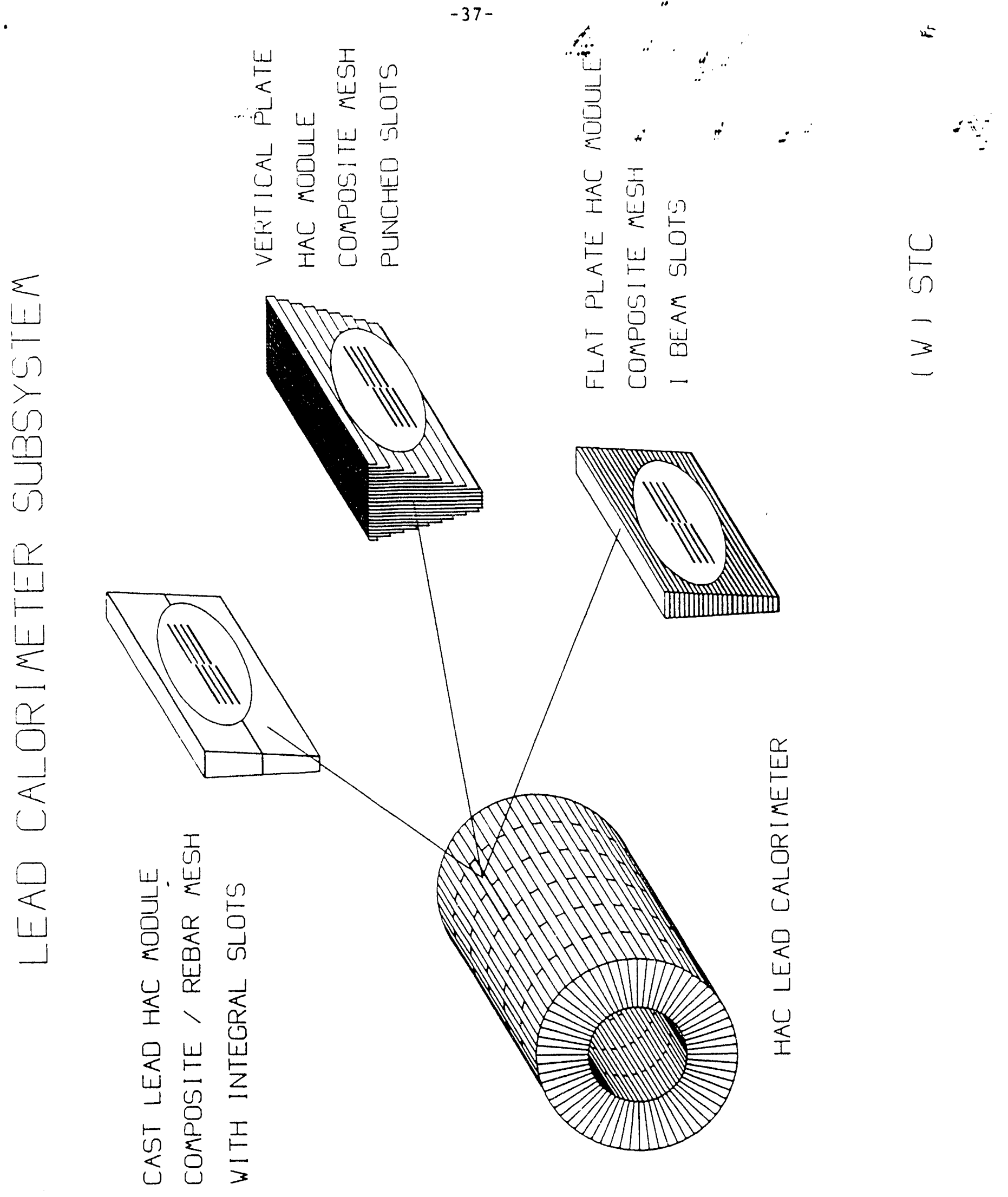

Figure 1.3 
$-38-$
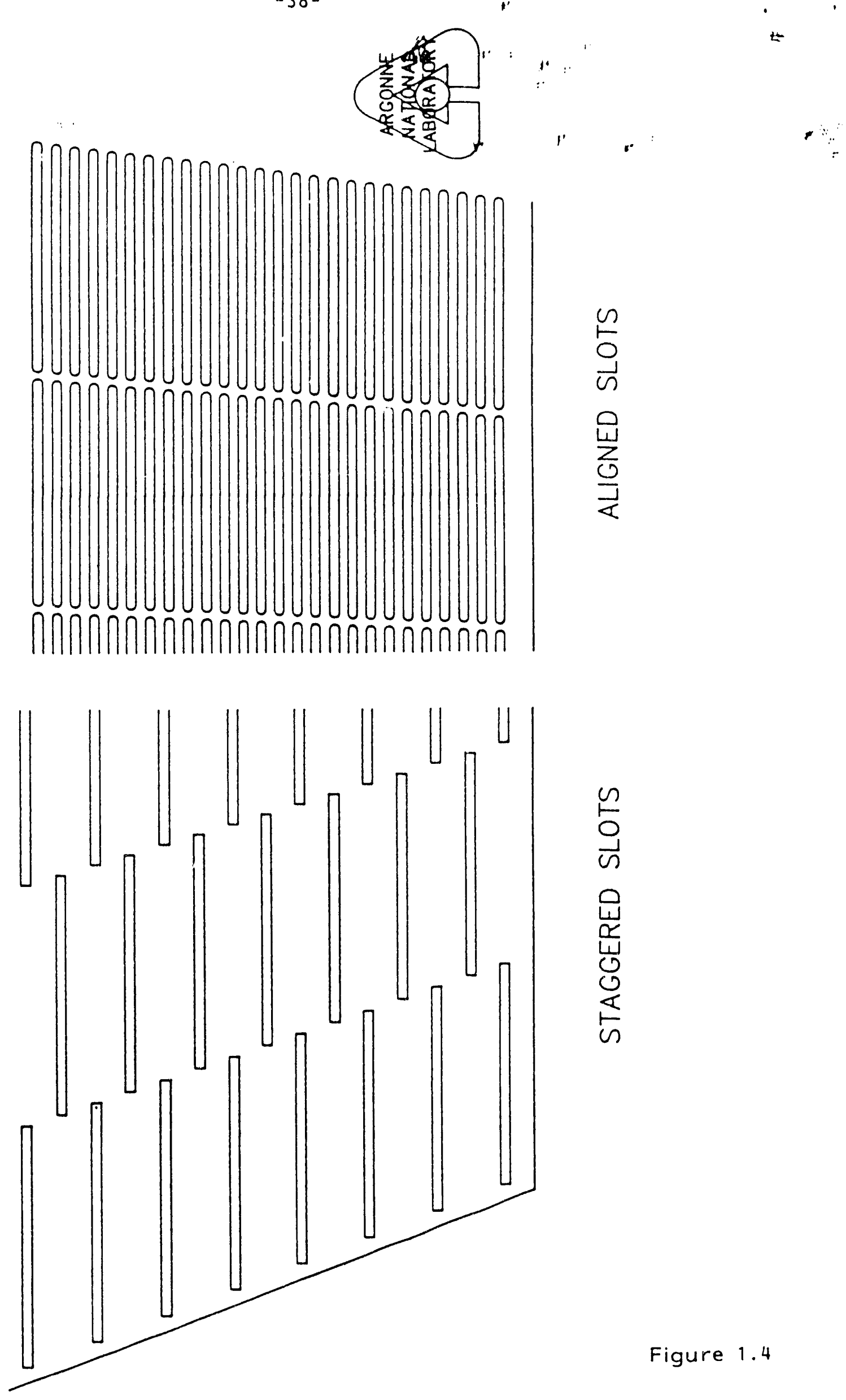

$n$
0
$\frac{1}{n}$
0
$\frac{1}{\alpha}$
$\frac{1}{0}$
0
$\frac{1}{n}$

Figure 1.4 
$\sum_{u}^{E}$

แ ㄴ (n) L Q

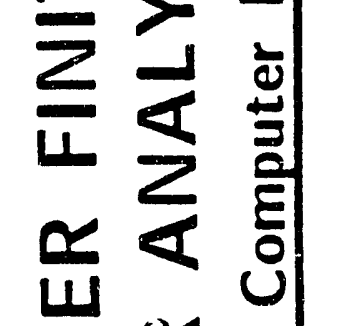

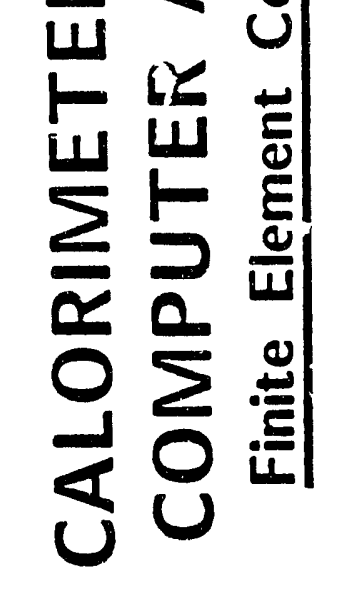

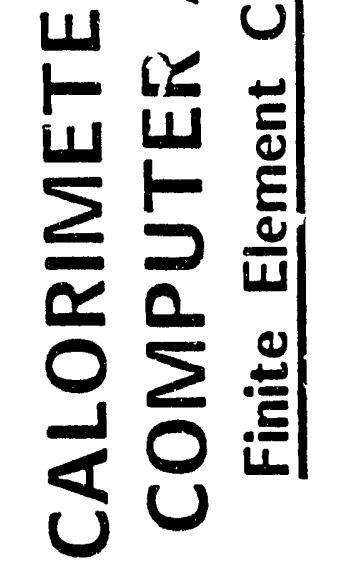

$\frac{\overrightarrow{1}}{\frac{\alpha}{\alpha}}$

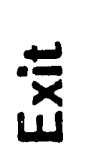

व
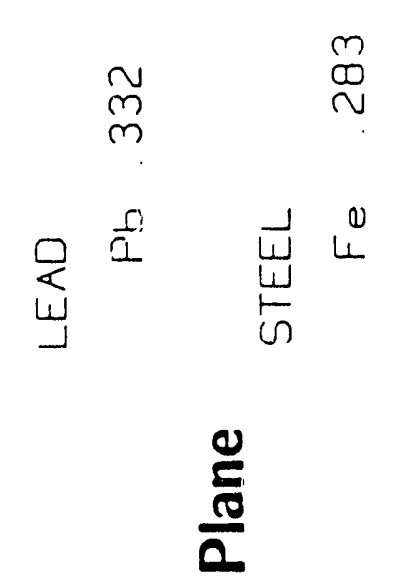

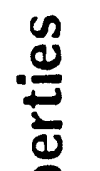<smiles>[CH][CH]</smiles>

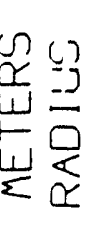

Figure 1.5 

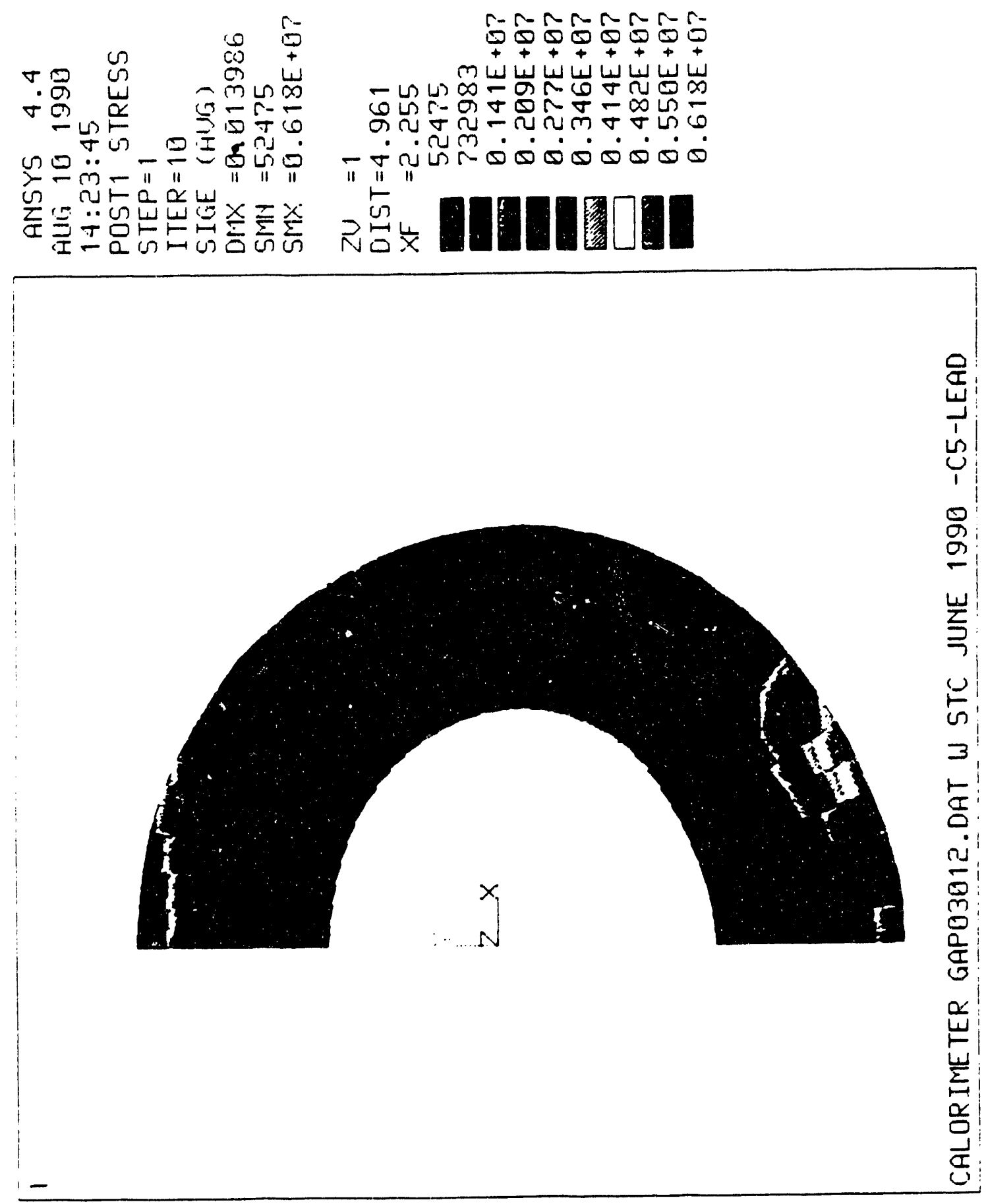

Figure 1.6 
,

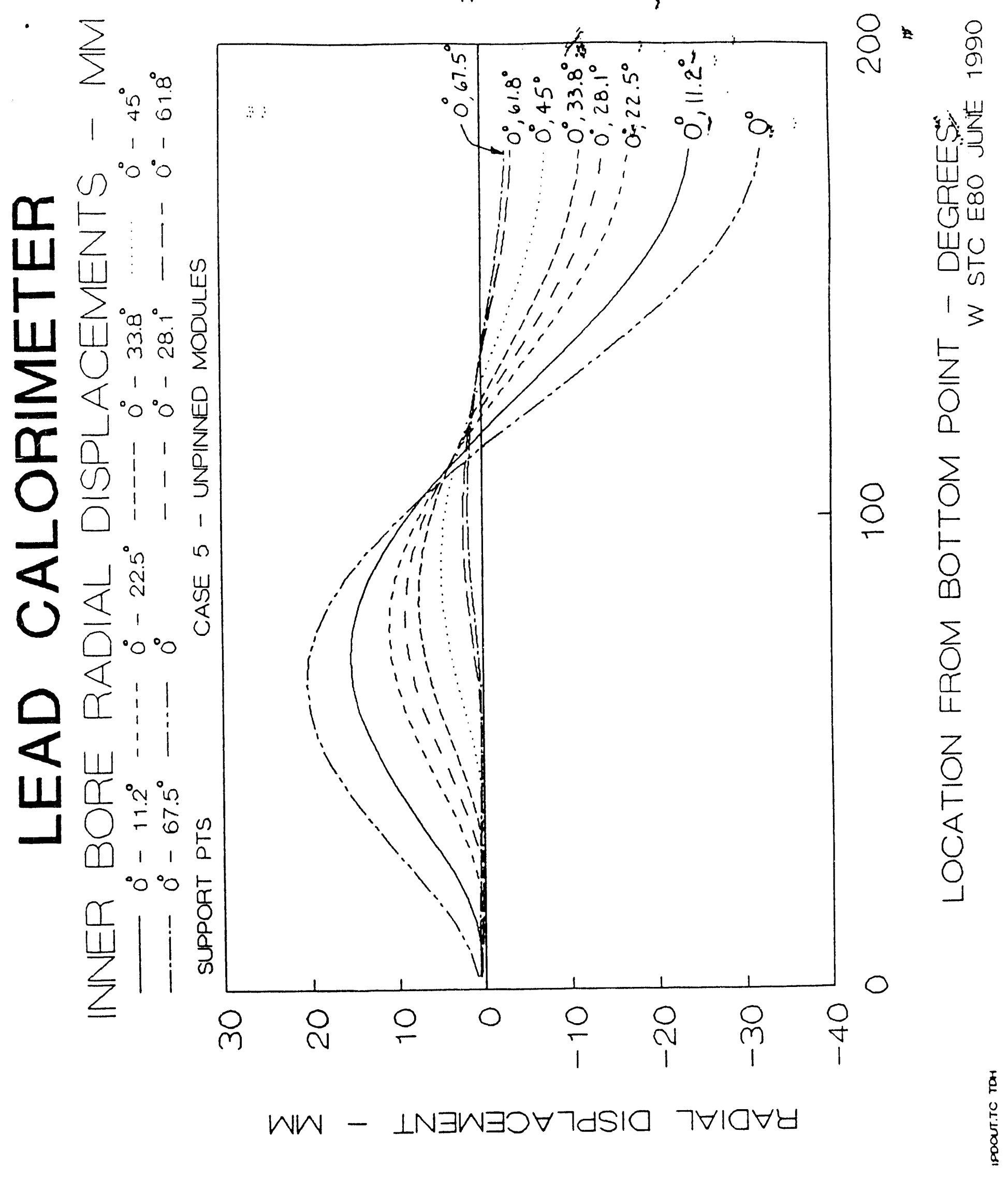

Figure 1.7 


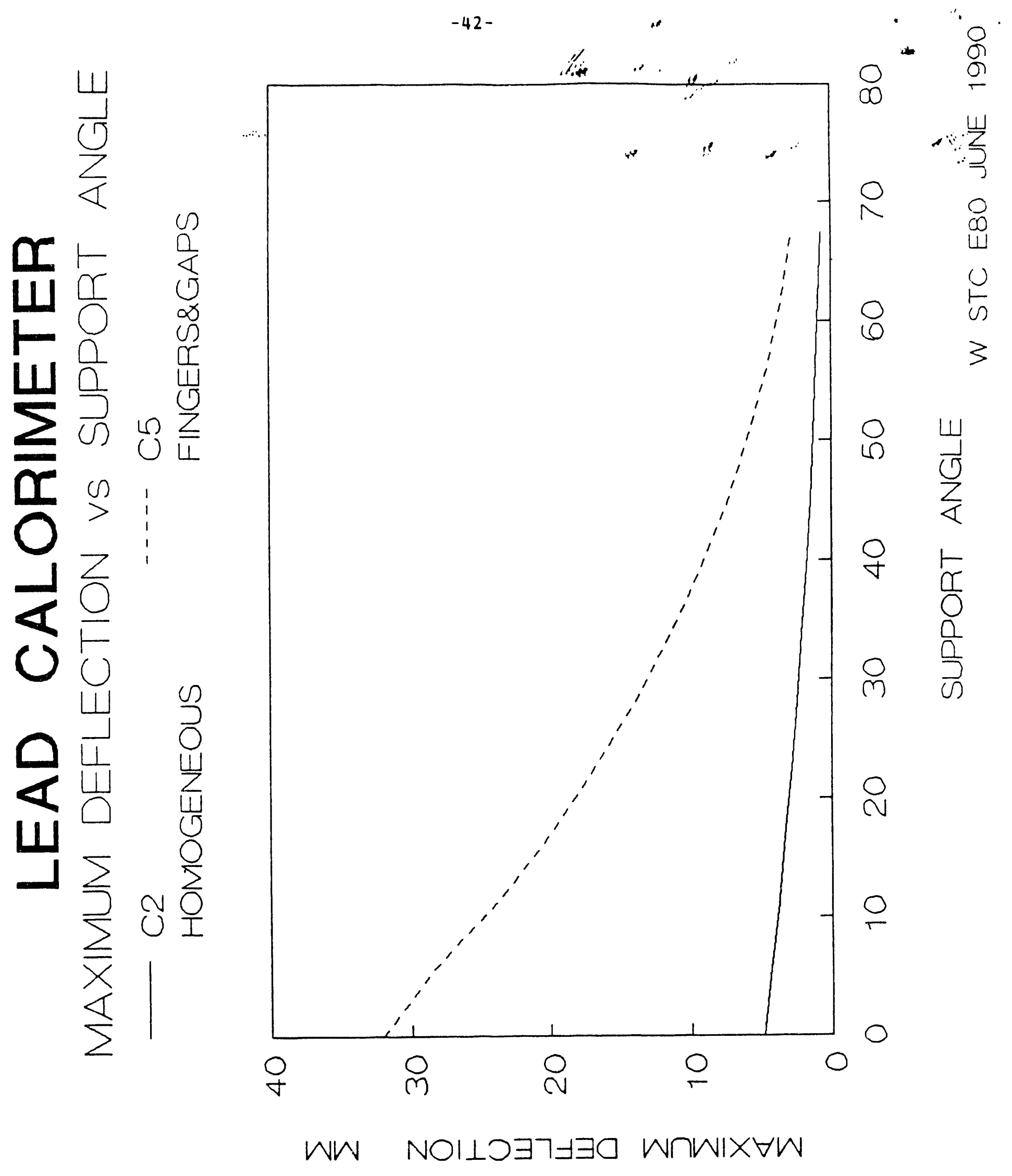

Figure 1.8 

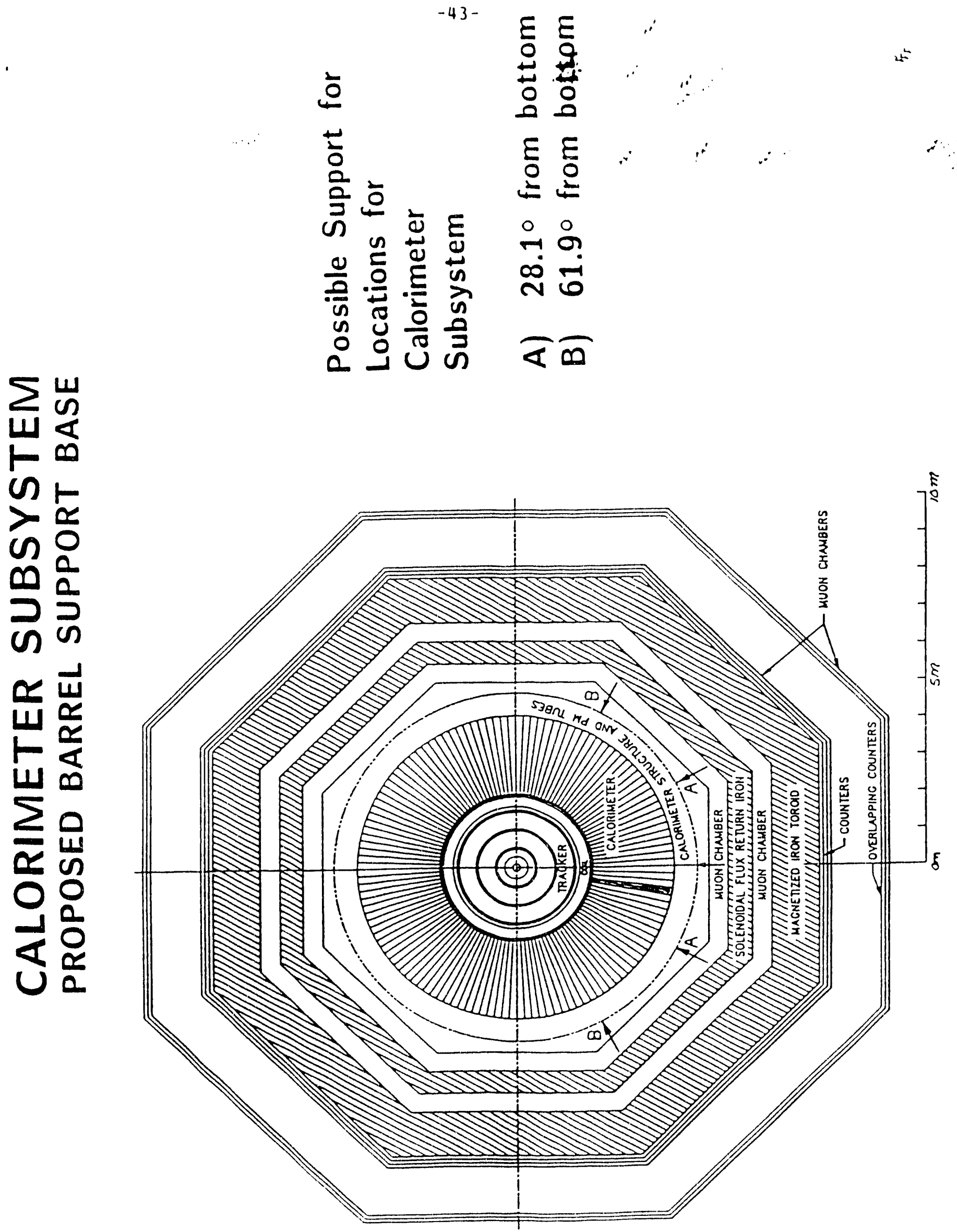

Figure 1.9 
$-44-$

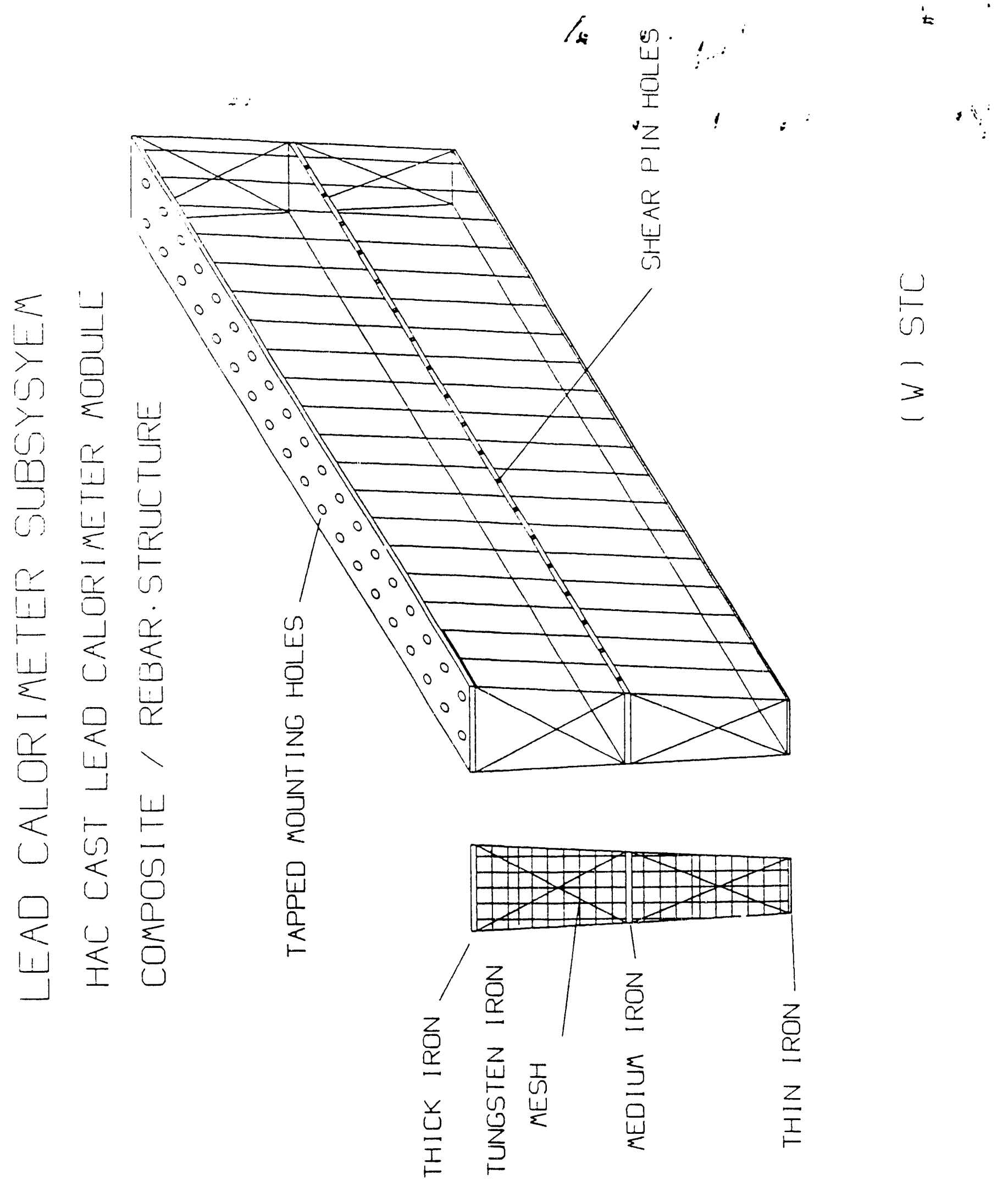

Figure 1.10 


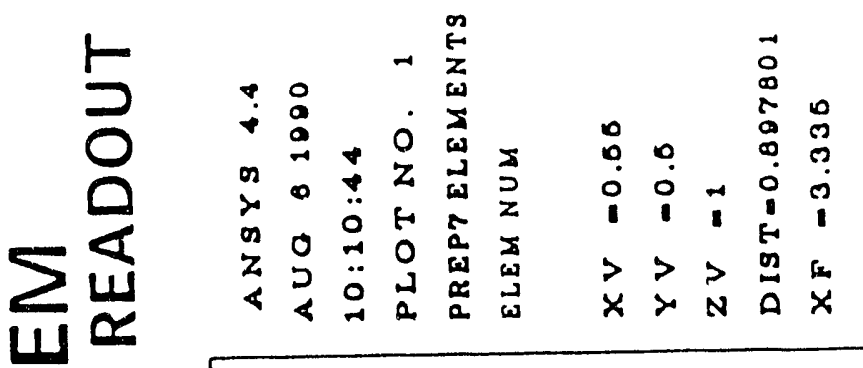$$
\text { 倠 : }
$$

$\alpha=$

W

$\sum 0$

$\sum \frac{\alpha}{\alpha}$

$0 \frac{0}{0}$

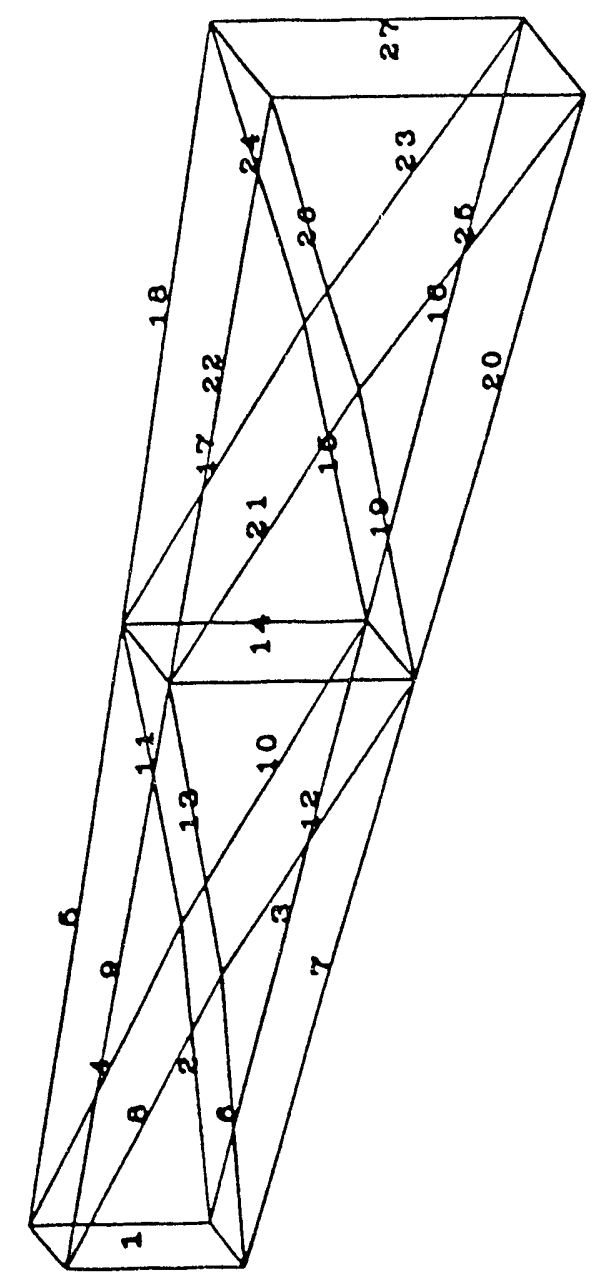




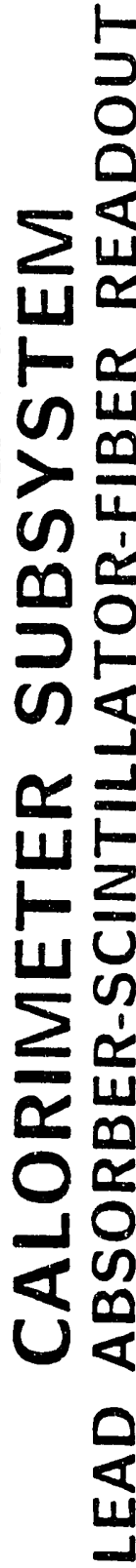

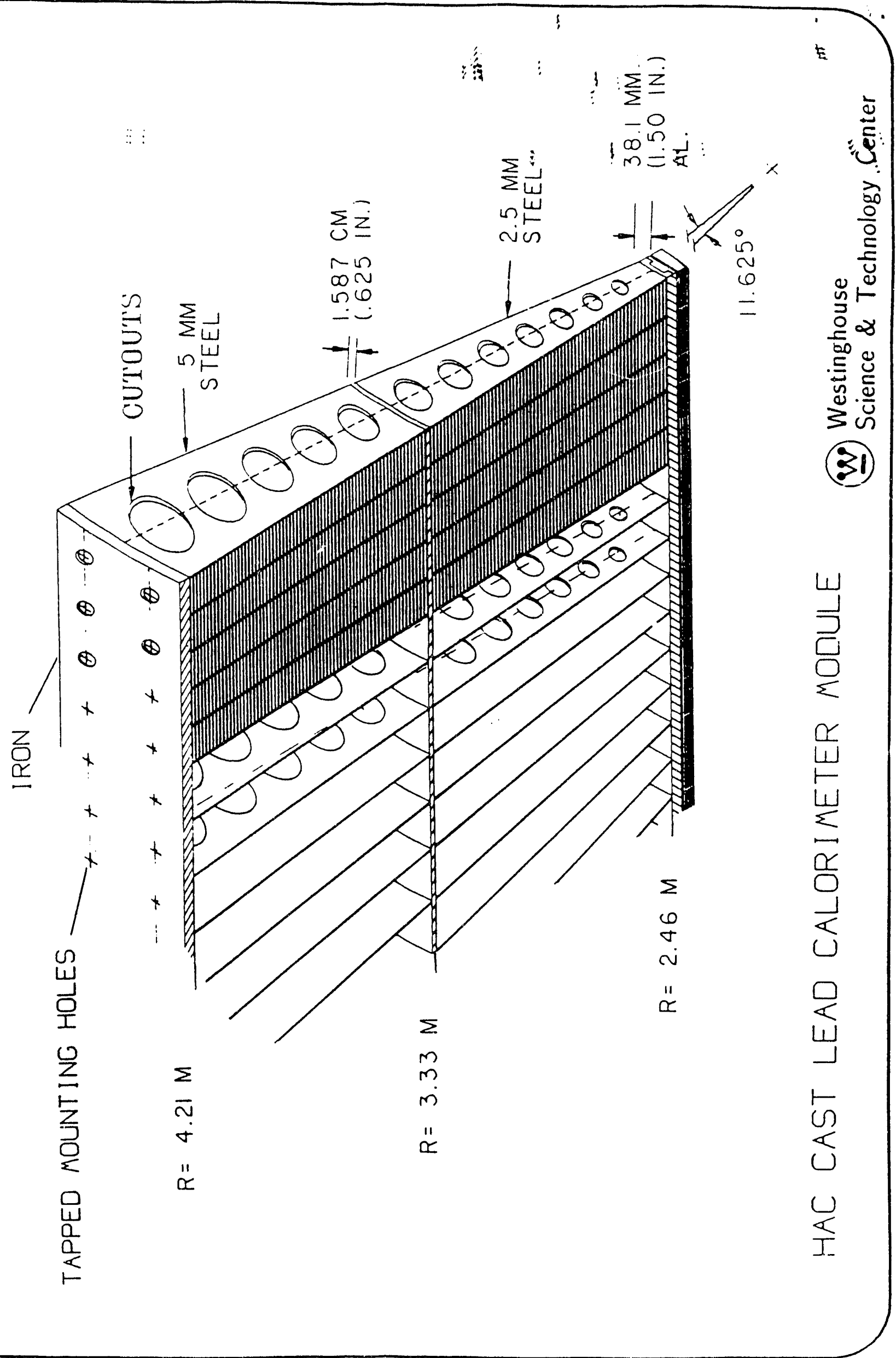

Figure 1.12 


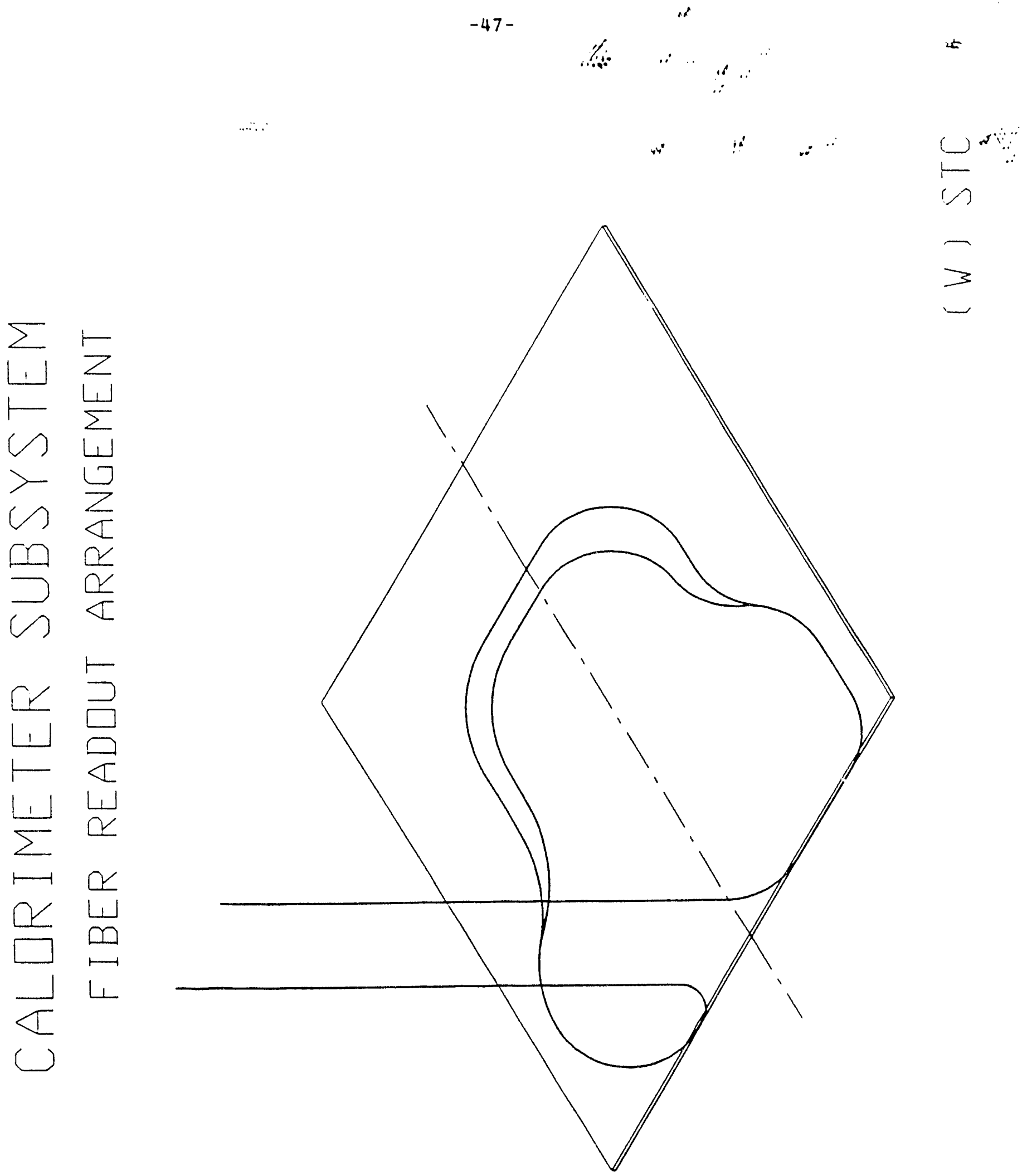

Figure 1.13 
$-48-$

fit

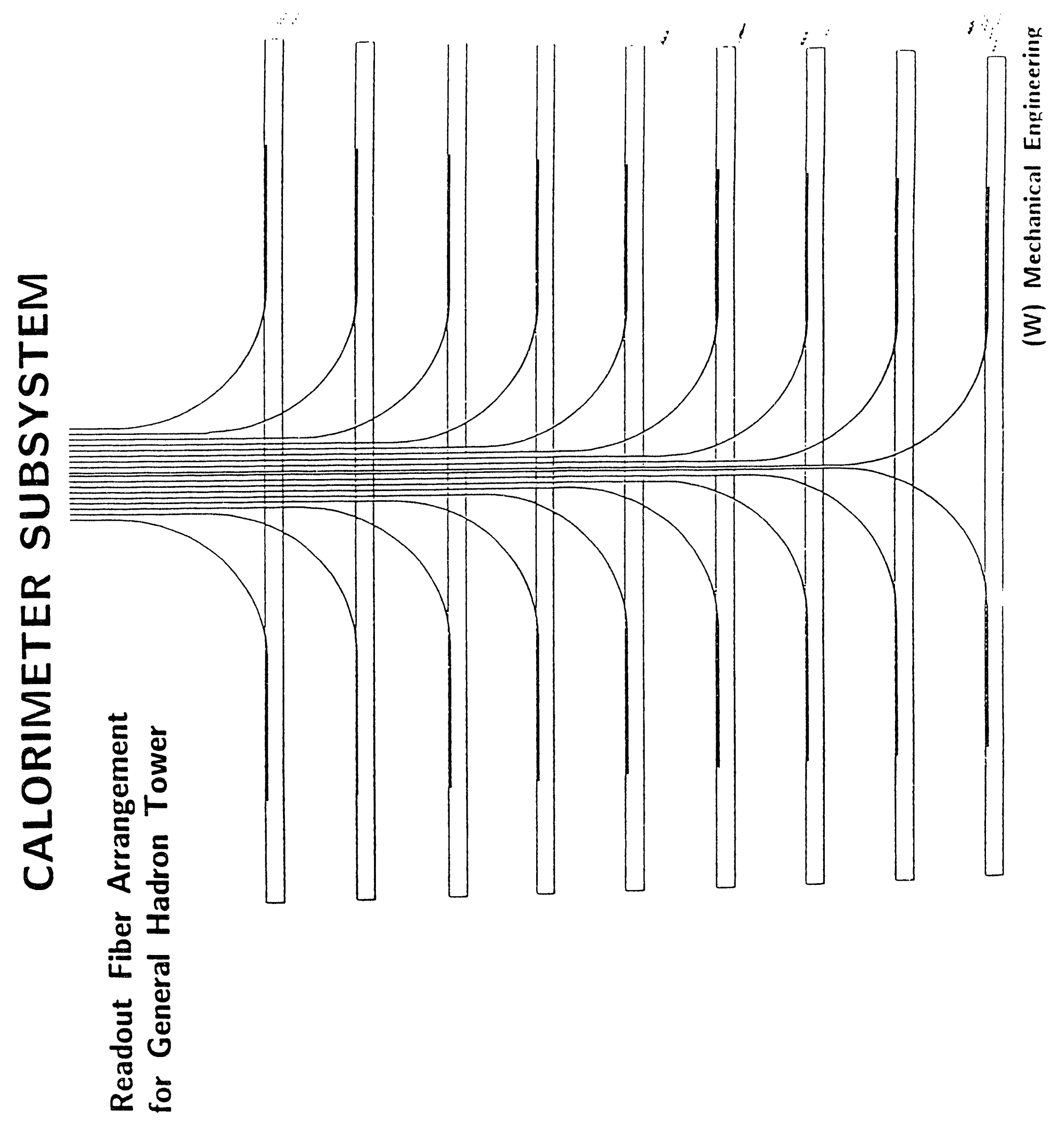

Figure 1.14 


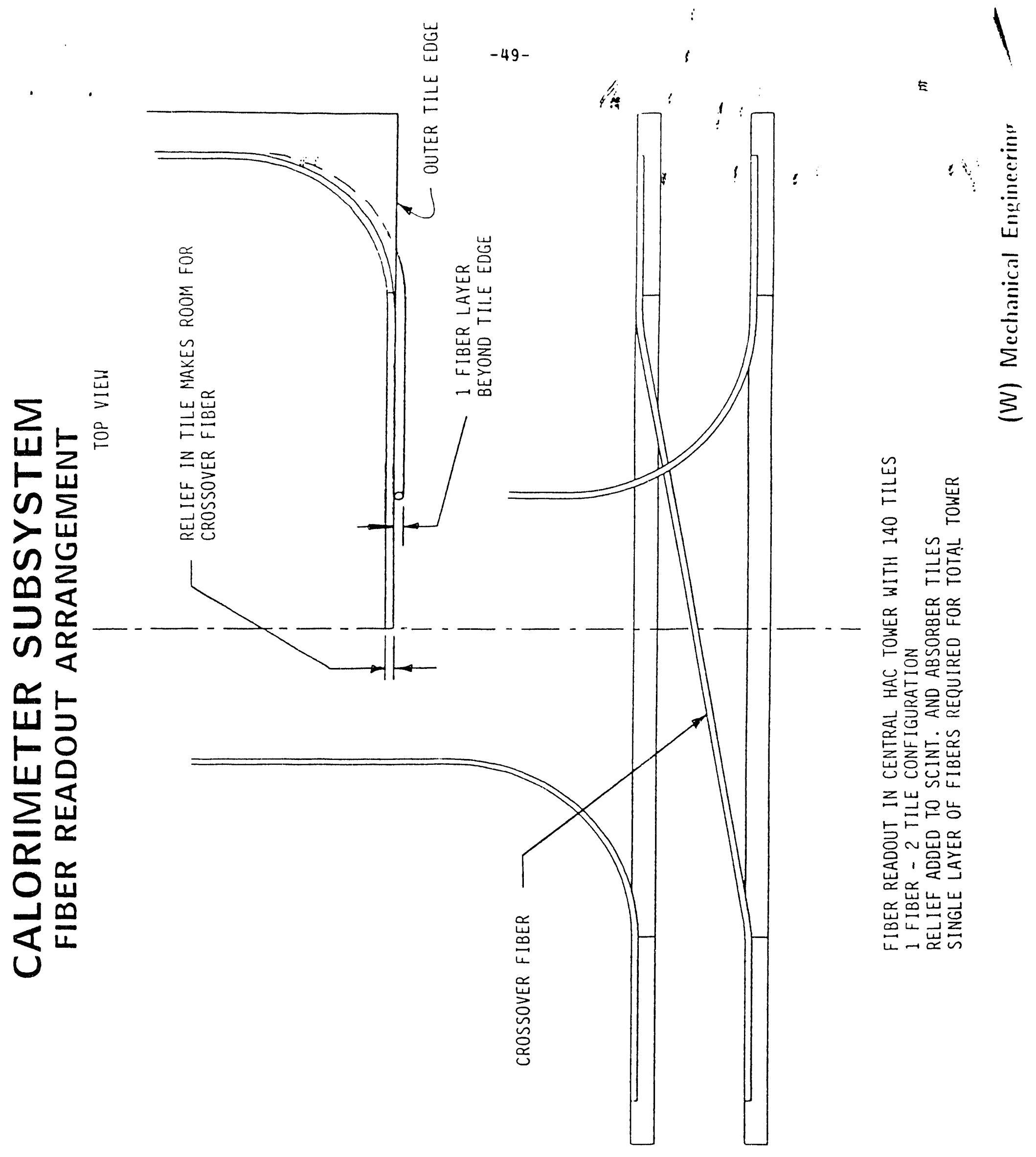

Figure 1.15 


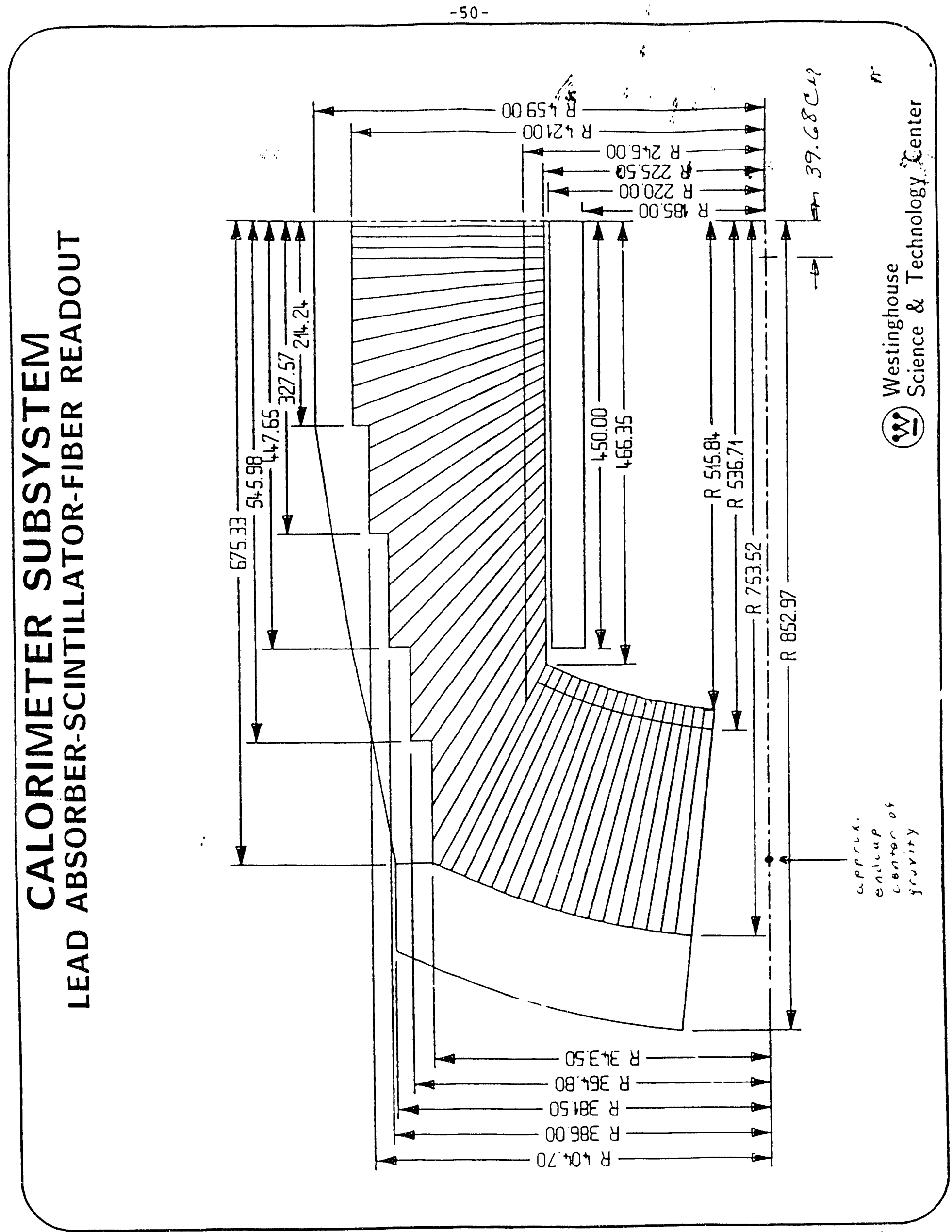

Figure 1.16 


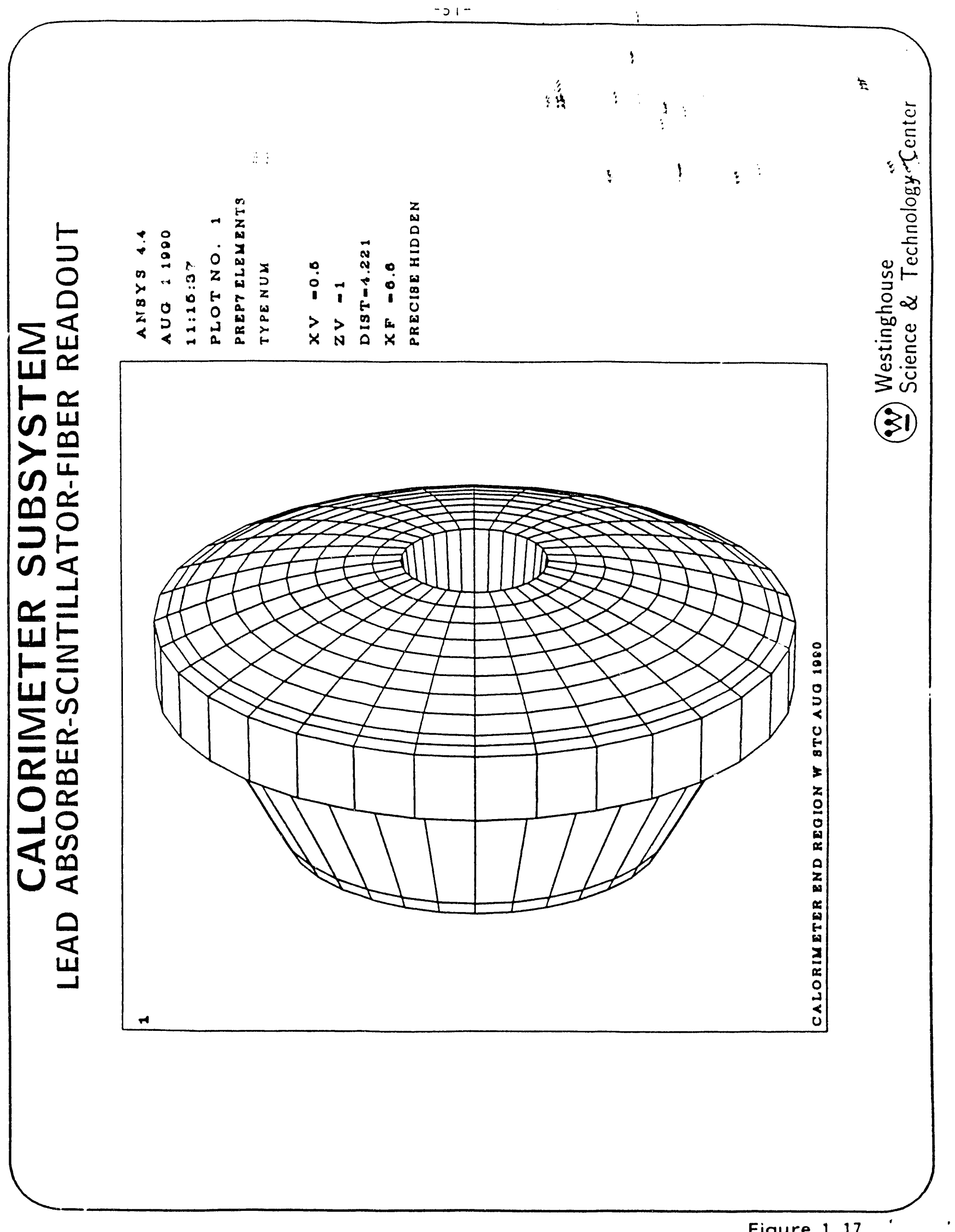




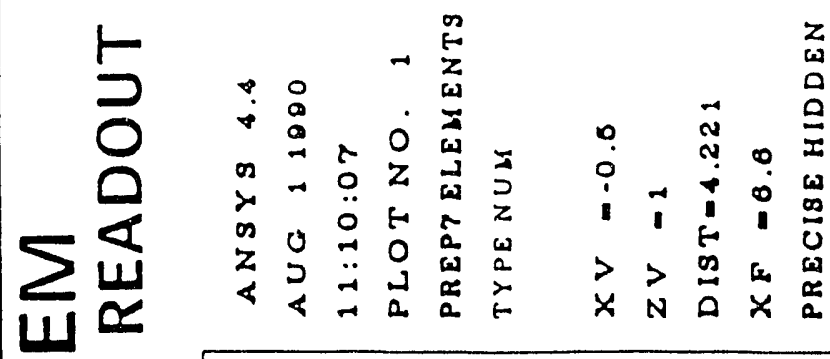
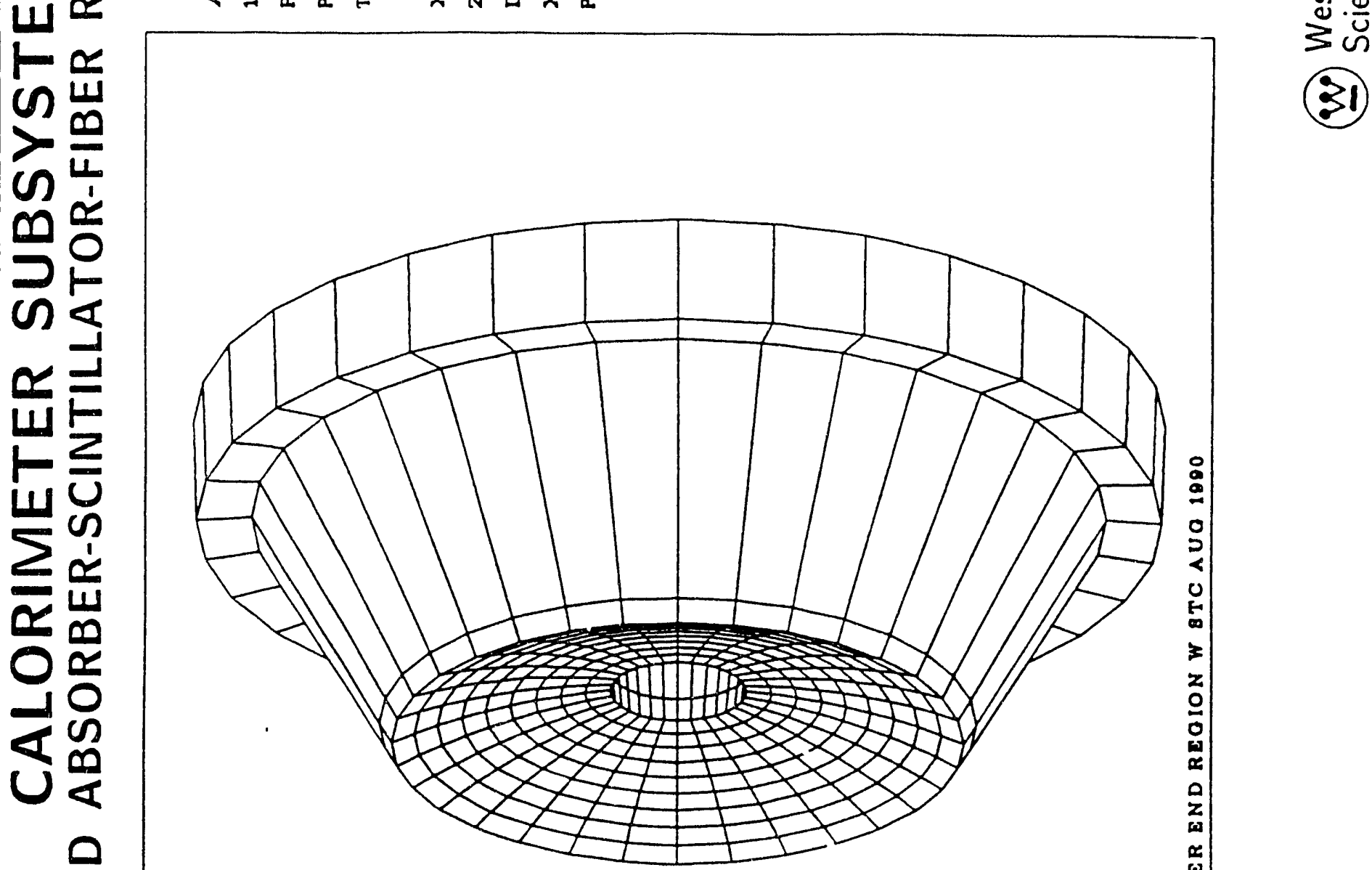

눈 
$-53-$

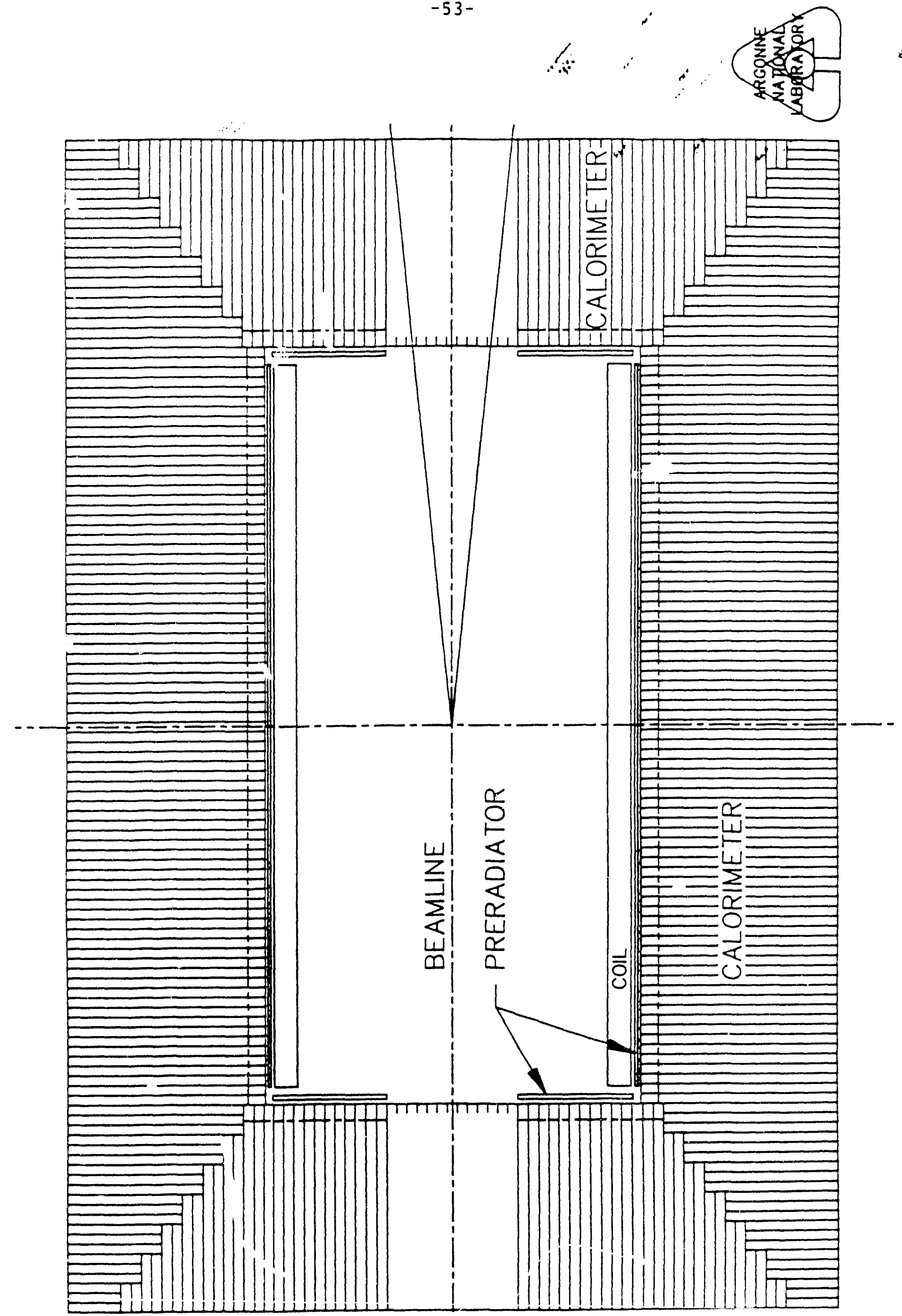

Figure 1.19 


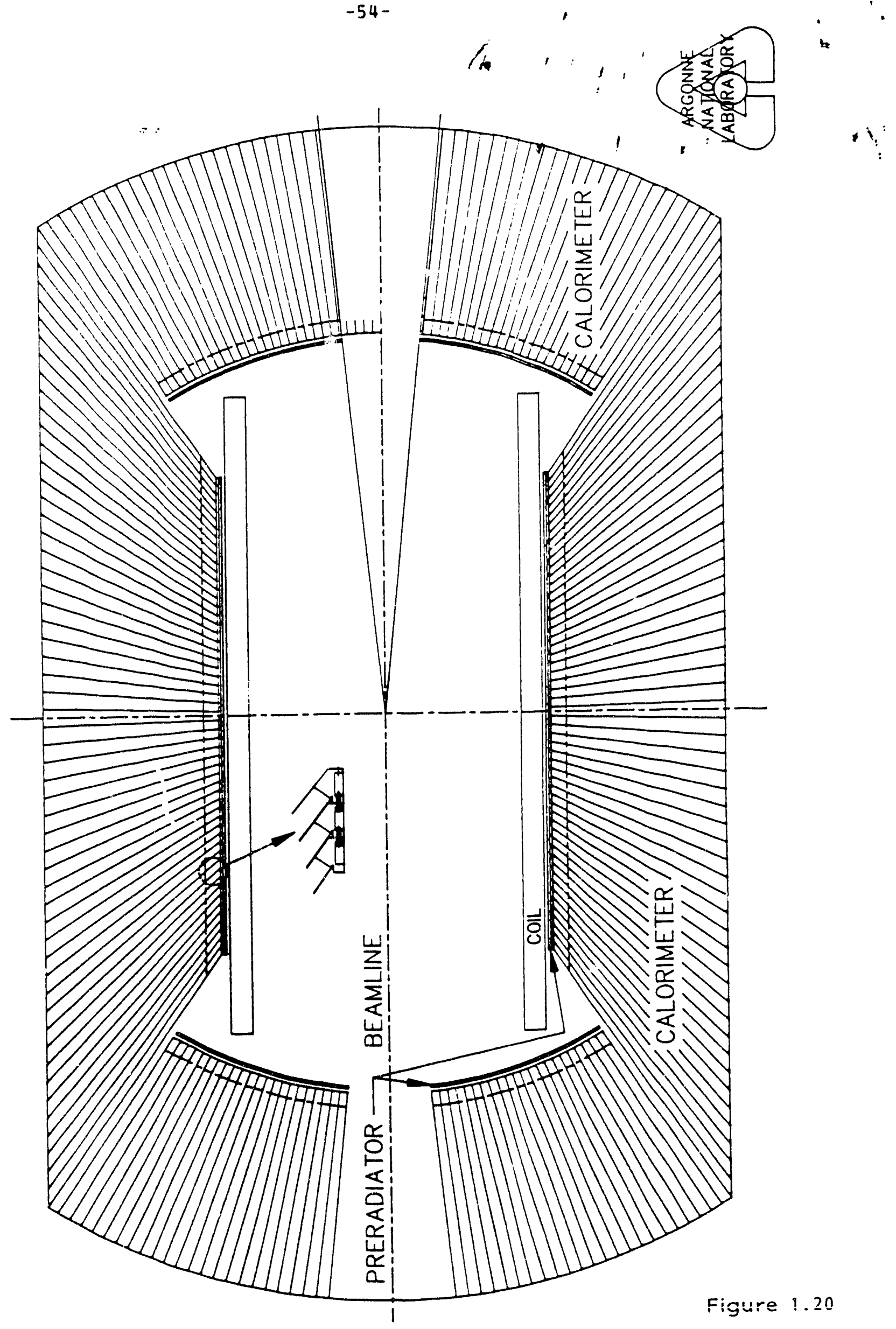



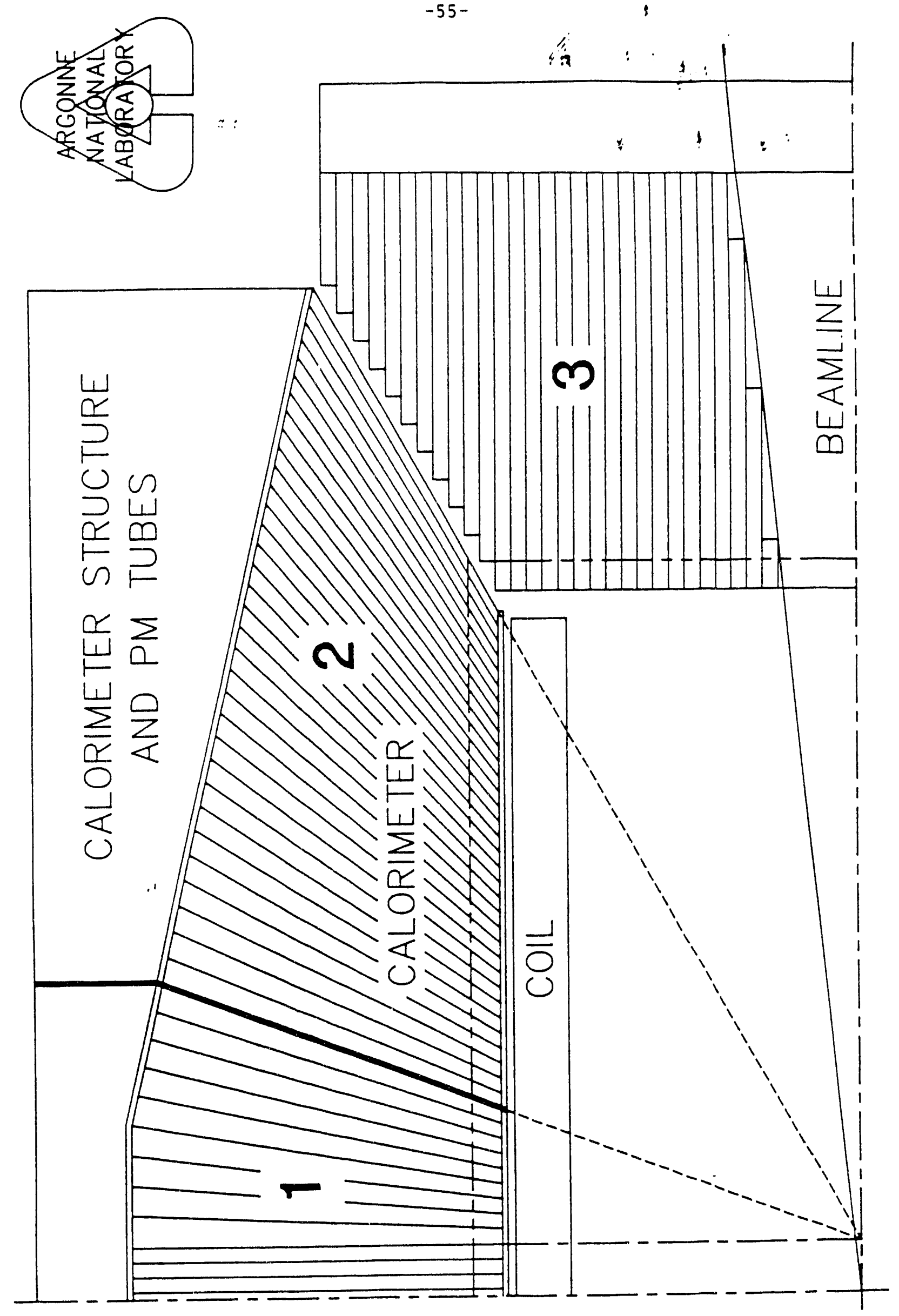

Figure 1.21 


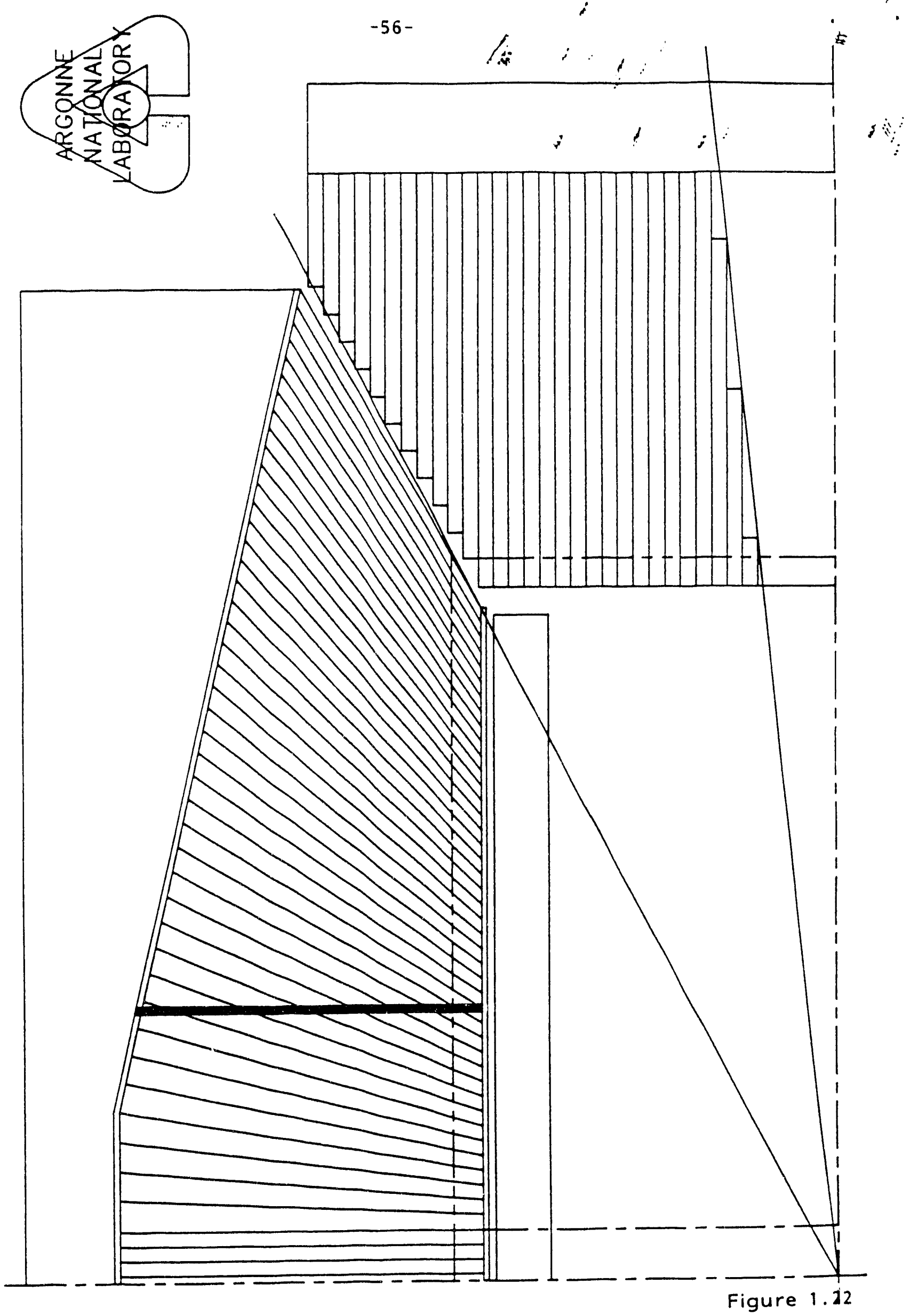


$-57-$
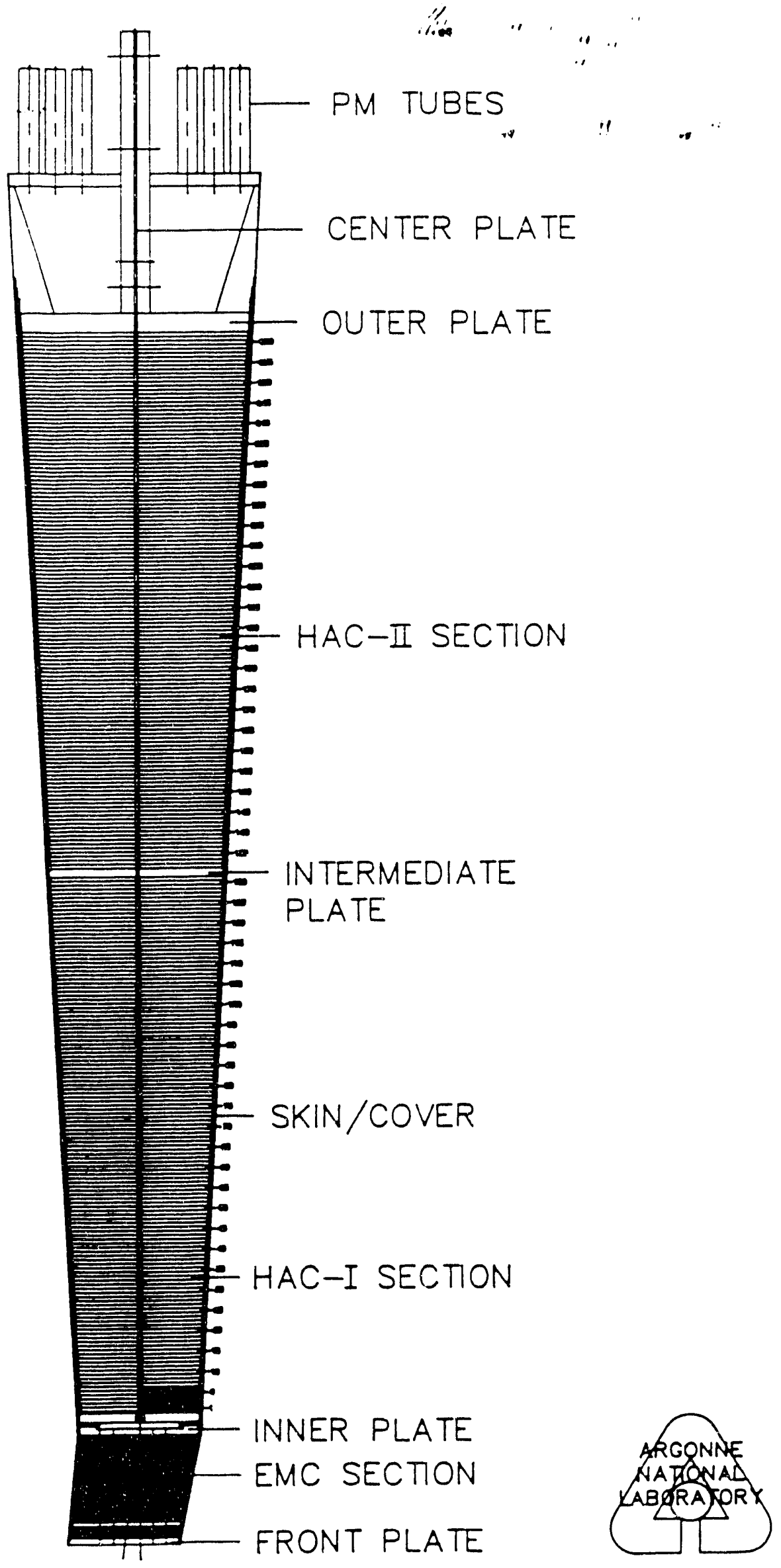

Figure 1.23 
$-58-$

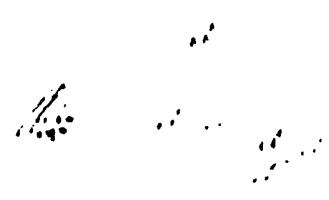

$\ldots .$.

$\because \quad \therefore \quad \therefore \quad \therefore$

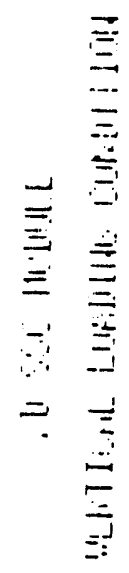

ت

$=$

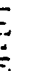

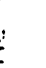




$$
\begin{array}{r}
1 \\
4 \\
4
\end{array}
$$

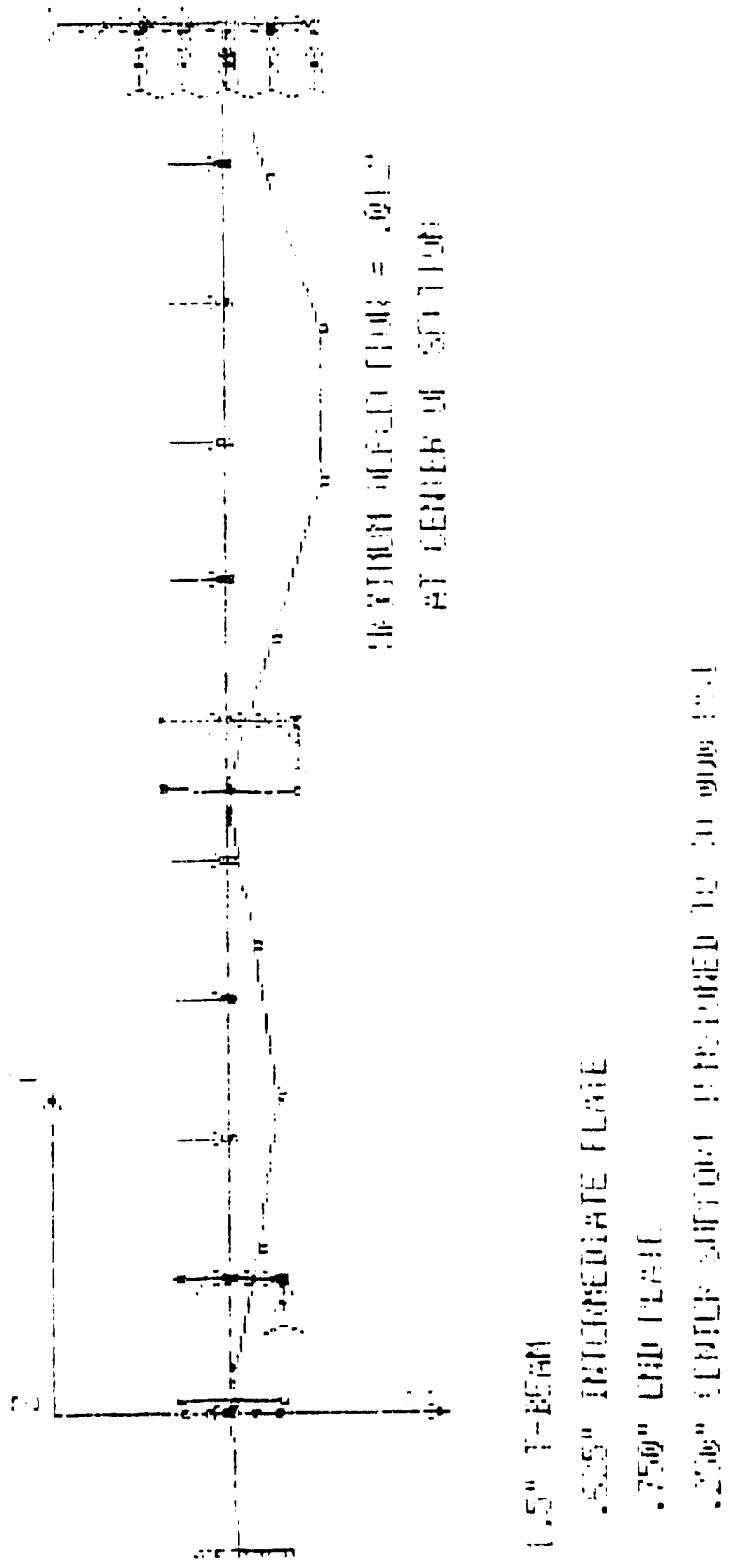

$=$
$=$
$=$
$=$
$=$

Figure 1.25 
$-60-$

le

$\sum_{\alpha}^{\mathcal{L}}$

슨

$\frac{\alpha}{5}$

$\frac{w}{\frac{1}{a}}$ 尚它

w

壳

w出

$z \frac{\alpha}{w}$

岀比

32

岗

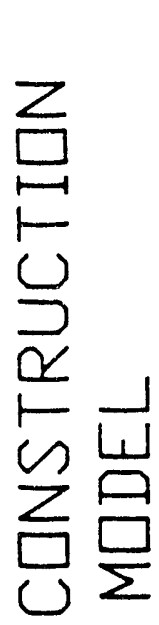

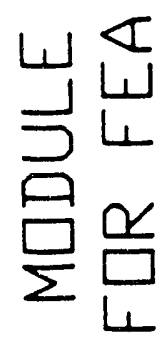

0

넌

in

凹日

选吉

ข

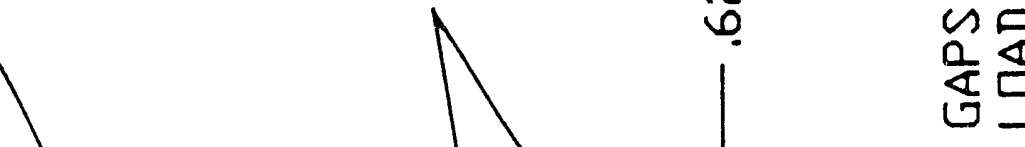

$\sum_{\alpha}^{\mathfrak{\alpha}}$

닐

in

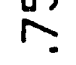

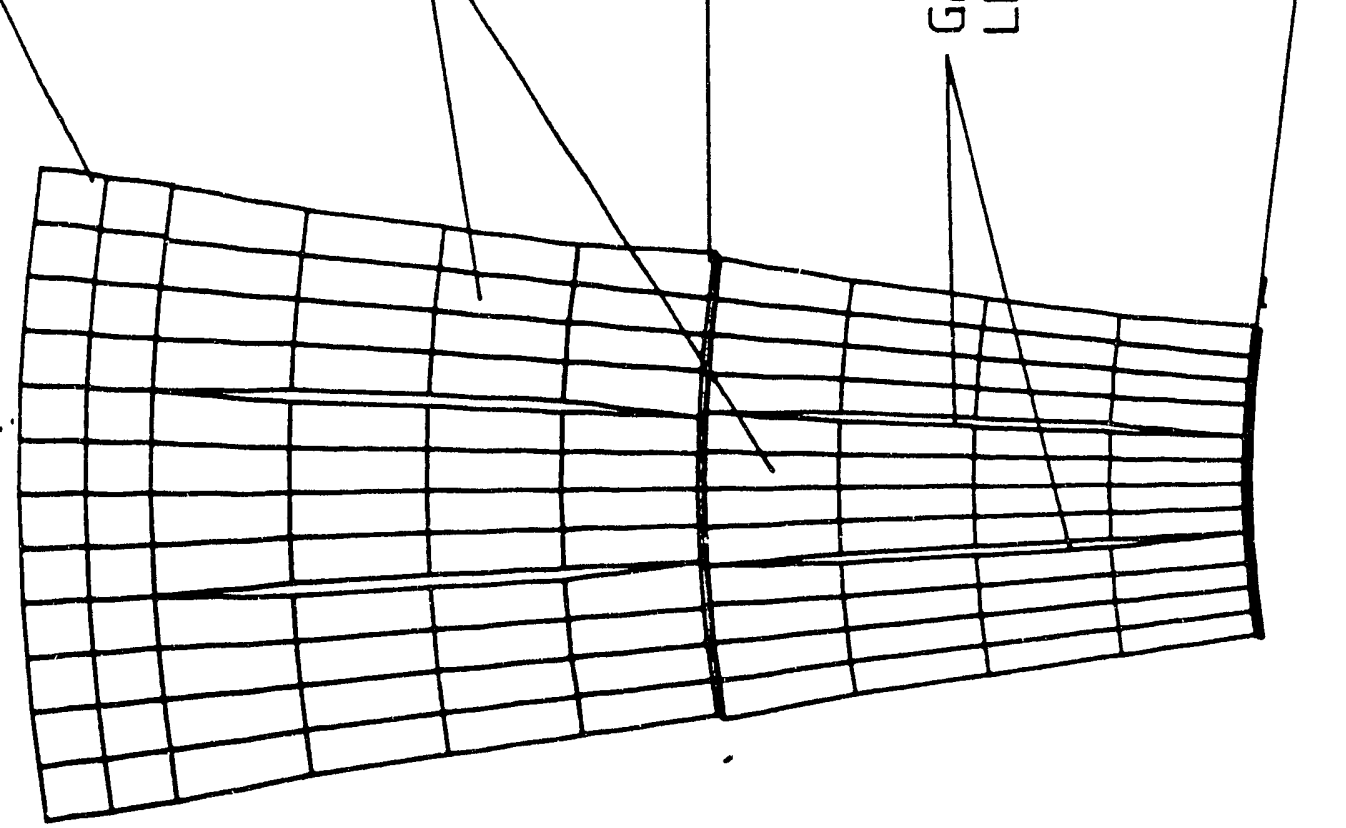

u

$\square$

U

Figure 1.26 


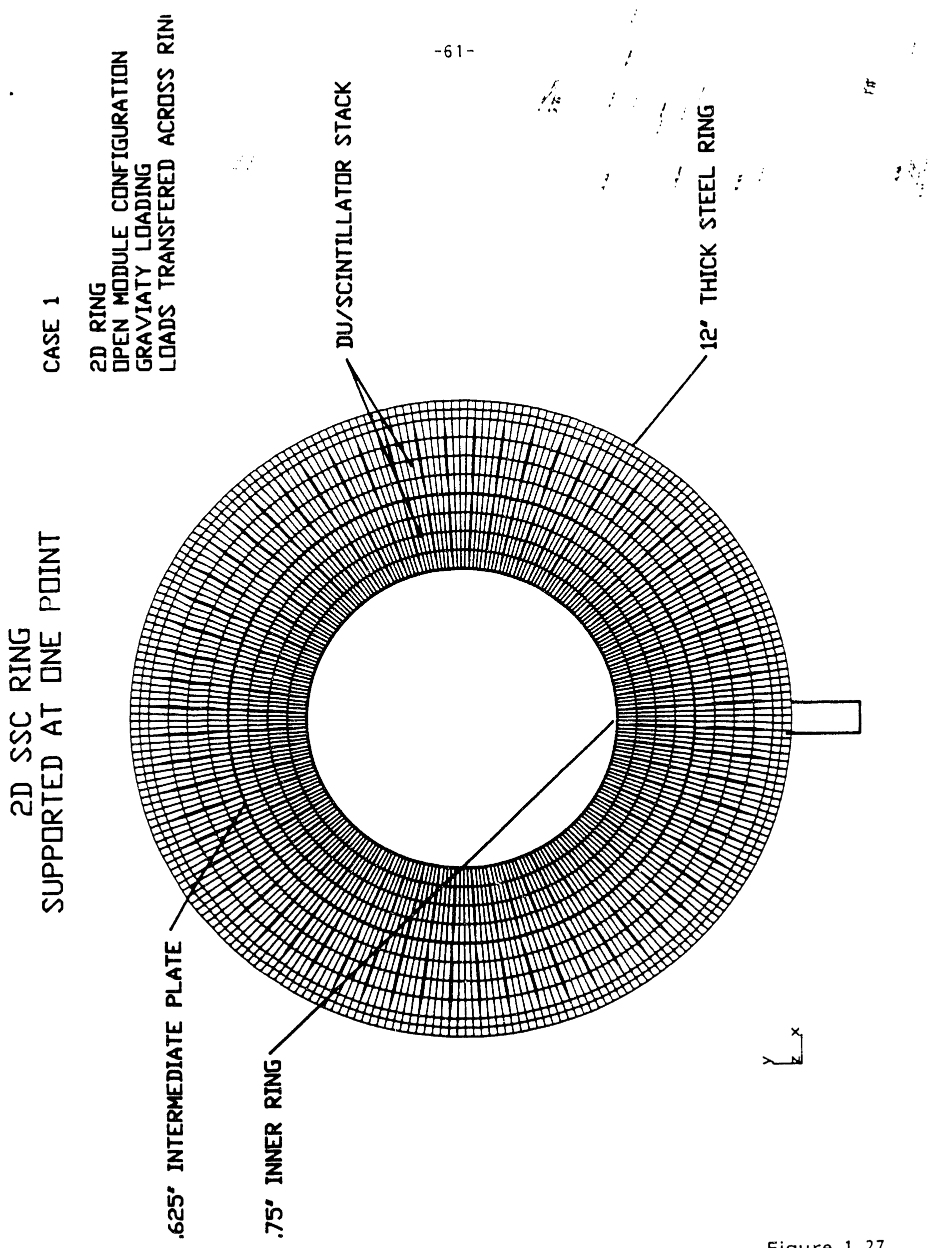

Figure 1.27 


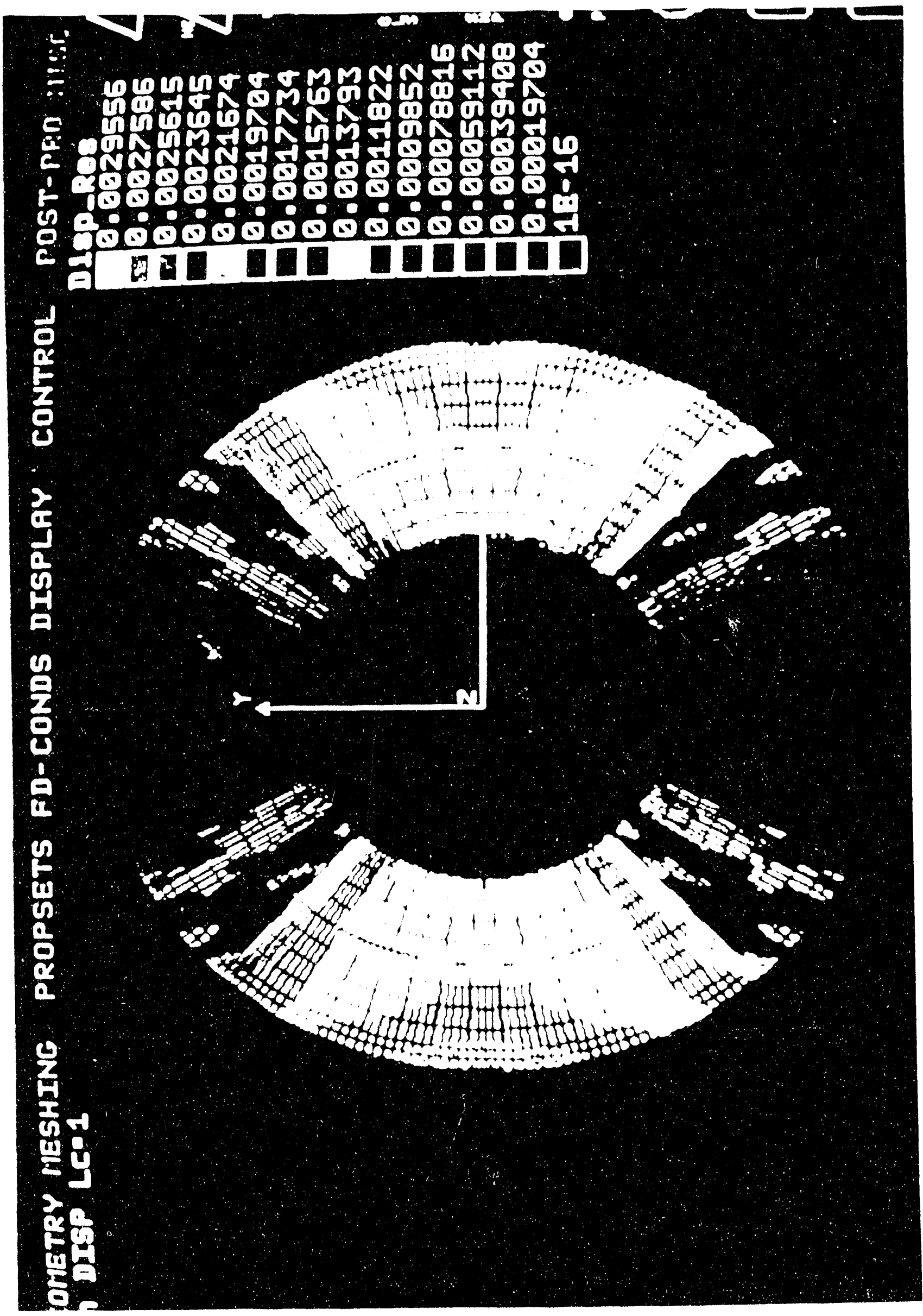




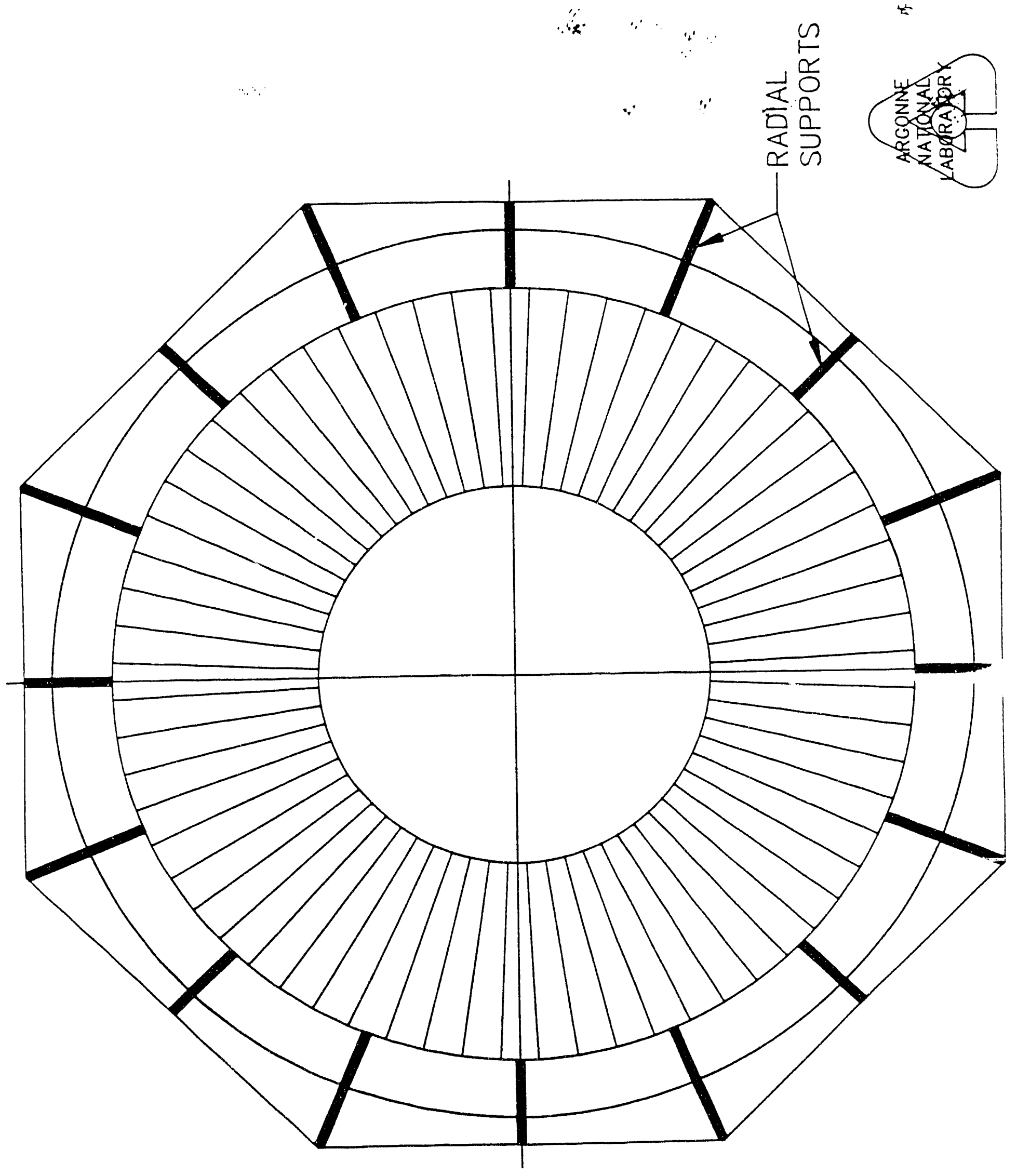

Figure 1.29 


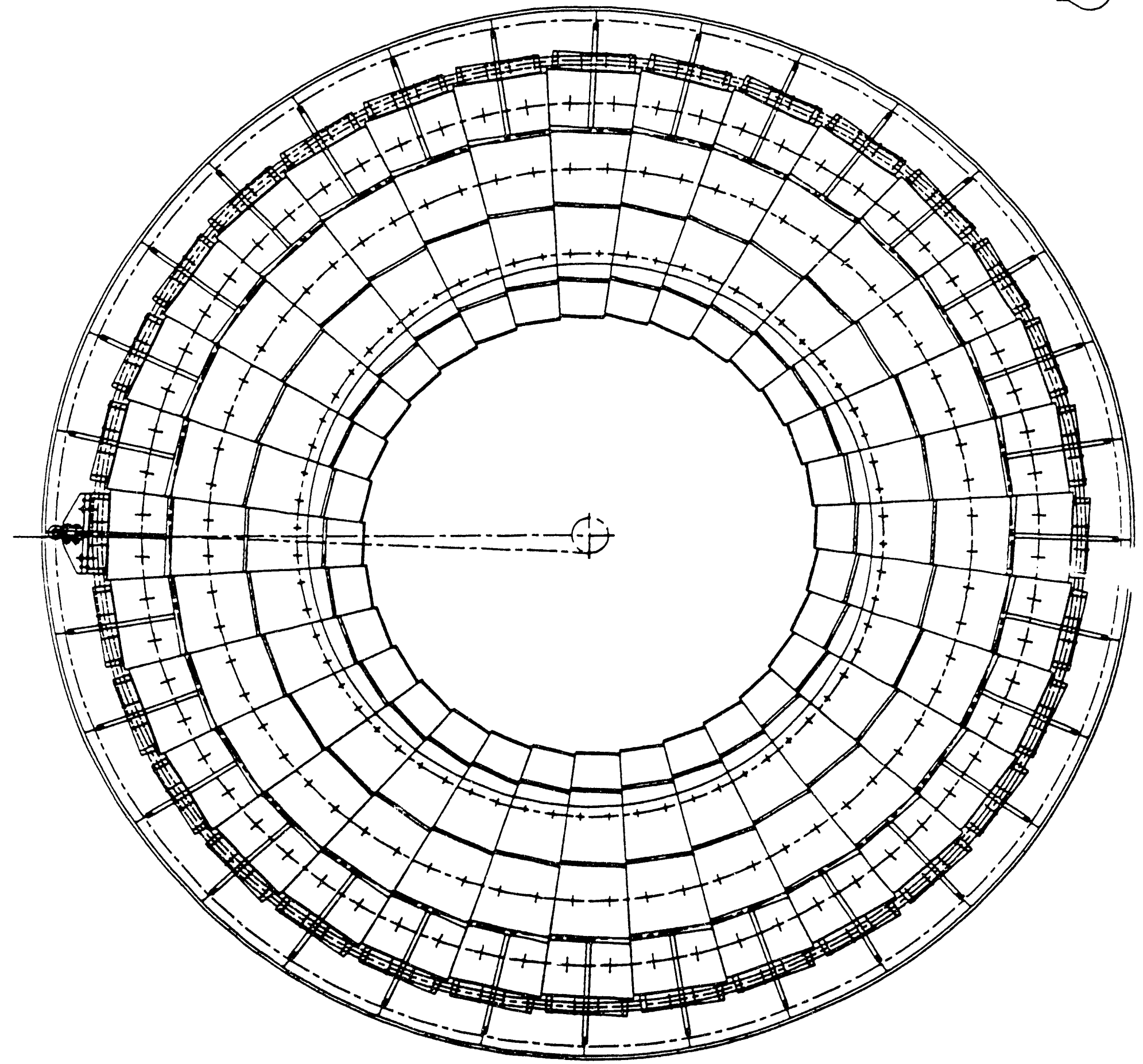

Figure 1.30 


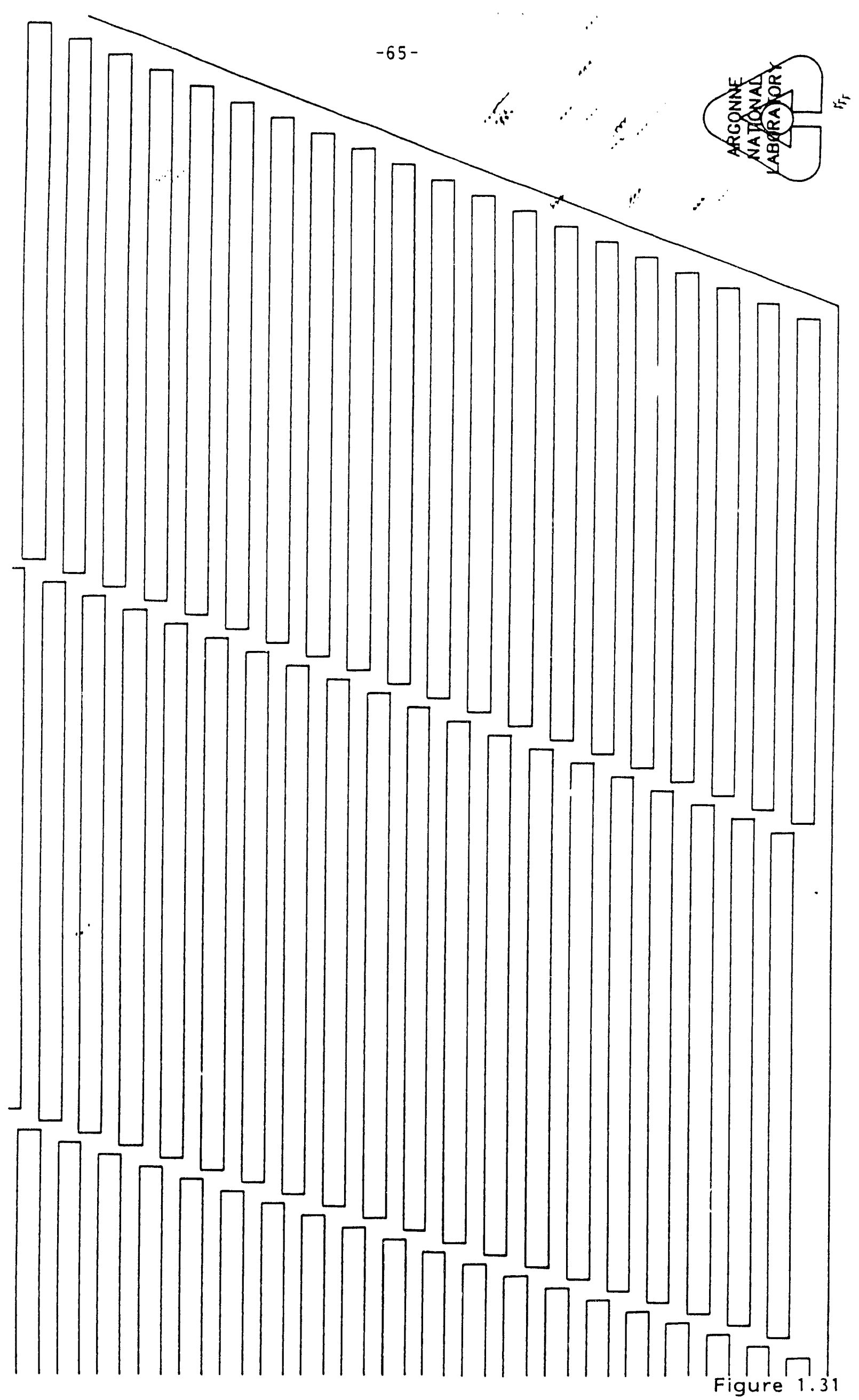



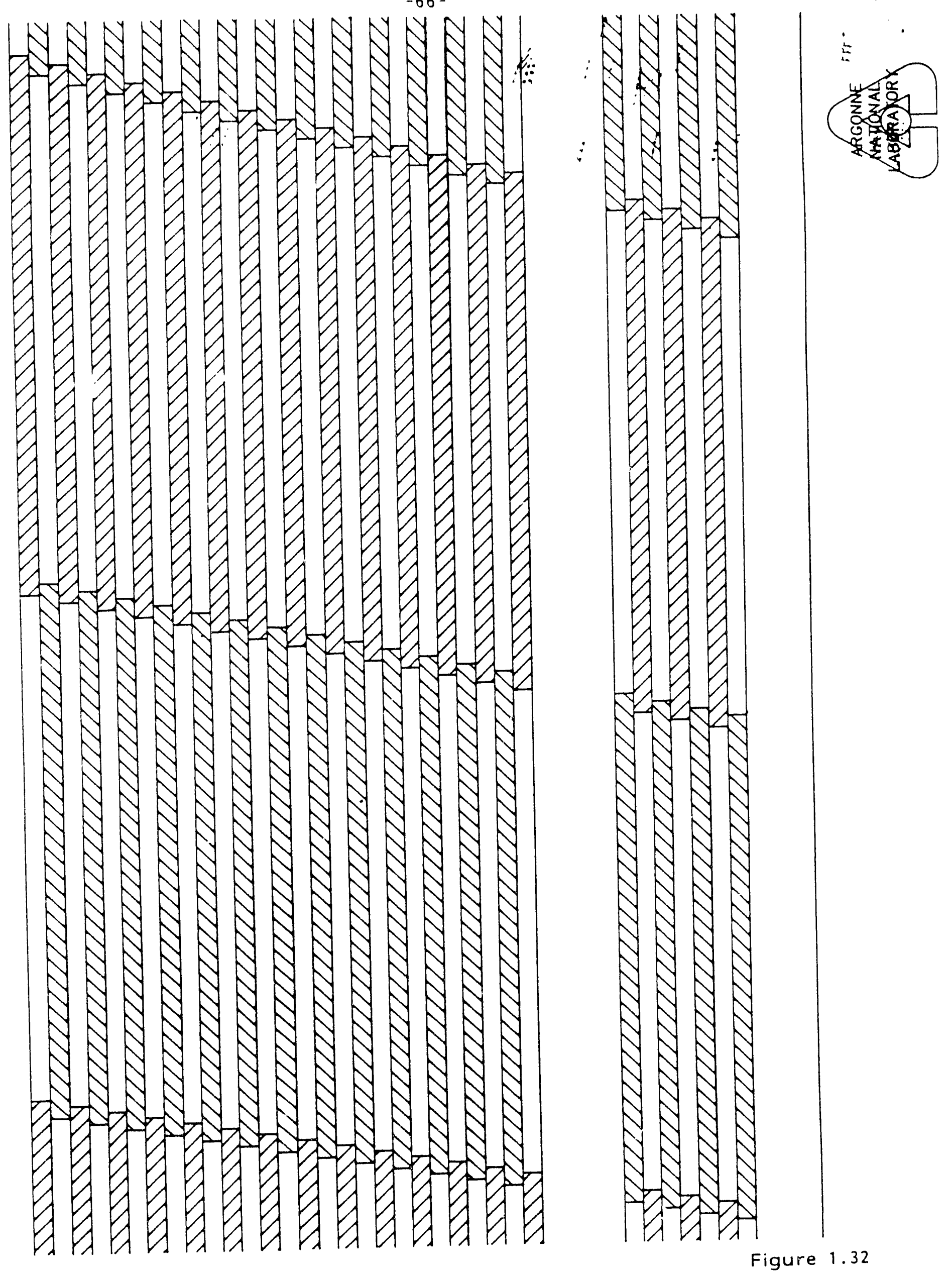
$-67-$

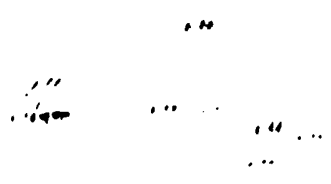
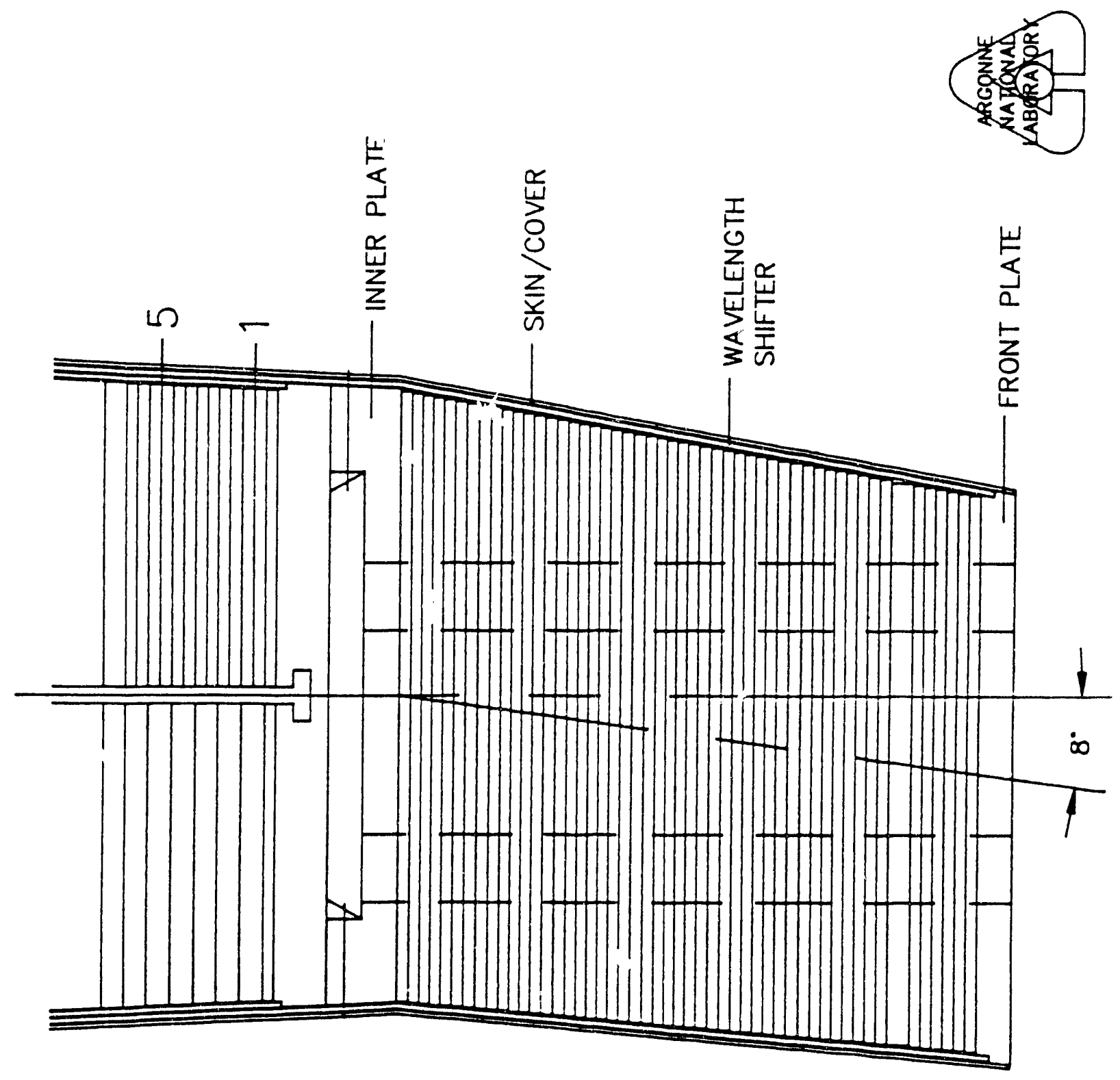

Figure 1.33 


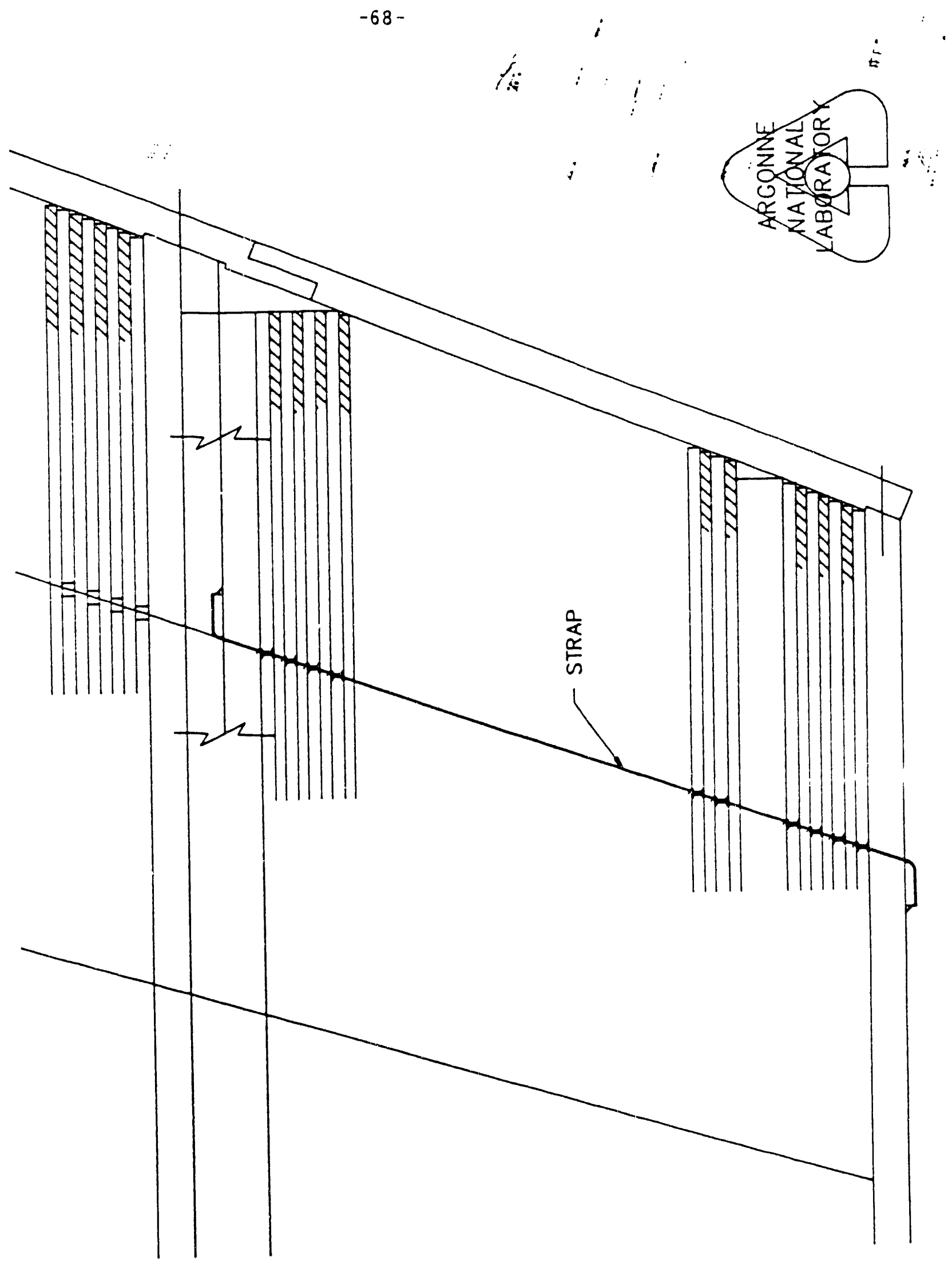

Figure 1.34 


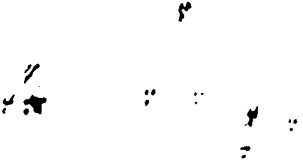

SECTION $1 \mathrm{~B}$.

LEAD BASED CALORIMETER MATER IALS STUDY

M. Burke, D. Hackworch, and R. Swensrud

Science and Technology Center

Westinghouse Electric Corporation, Pitcsburgh, Pennsylvania 15235

As part of the mechanical design studies for the lead based calorimeter, a materials study was initiated to evaluate lead based materials for implementation in the calorimeter structural design. The objectives of the study were: 1) to compare the mechanical properties of candidate materials with the mechanical loading requirements to determine acceptable materials, and 2) to analyze the cost, fabrication and manufacturing issues associated with these materials in order to determine the optimum material for the application. The mechanical requirements for the calorimeter were derived from the ongoing mechanical analysis and were updated periodically as improved designs and analyses were developed. Essentially all the work on both the mechanical design and the macerials study for the lead based calorimeter was performed at the Westinghouse Science and Technology Center under subcontract from Argonne National Laboratory. The results of the study are described below. It is concluded that lead based composites are a mechanically feasible and cost effective approach to producing a lead based calorimeter.

The major issue that arises from the use of lead based absorbers in a calorimeter is the low mechanical performance of these materials. Lead is a mechanically oft and weak material. Also, because lead has a melting point of $320^{\circ} \mathrm{C}$, it exhibits a time-dependent mechanical response at room 
cemperature. That is, at room cemperature under constant load, lead will slowly deform. The loads that the calorimeter materisis must witristand are determined by the materials of construction, since these forces are determined principally by the overall weight of the structure, and by loadings that are imposed to assemble the structure. Viable leald based materials would be those that exhibit elastic stiffness and time dependent strength properties that exceed the loading imposed by the calorimeter structure.

Several lead based materials were considered under the scope of this study. The materials included lead, low alloy content lead alloys, and particulate, fiber, and structurally reinforced lead composices. The following chart describes the materials considered in the study and highlights che generic advantages and disadvantages of each class of materials.

\section{Class}

Pure Lead

Lead Alloys

(low alloy content)

Particulate Reinforced Lead Composites

Fiber Reinforced Lead Composites
Advantages

Cheap, plentiful, readily available, easily worked and fabricated, homogeneous

Improved strength and creep resistance compared to pure lead, homogeneous, machinable

Improved stiffness and creep resistance, homogeneous, macisinable

Superior stiffness and creep resistance

\section{Disadvantages}

Mechanically soft, creeps at low temperature

Still not high strength

May be problems joining pieces

Probably requires integrated material/component manufacturing approach, anisotropic, may te problems joining pieces and fabricating 
In summary, commercially available lead and its alloys are expected to display inferior but homogeneous mechanical properties, while" the composites' are expected to exhibit superior but nonhomogeneous and anisotropic mechanical properties. Moreover, composites are also expected to require somewhat more complex fabrication and manufacturing procedures.

The mechanical properties of the candidate materials were collected and compared with the load bearing requirements developed from mechanical analyses. The goal was to identify materials that could support gravity loads without distortion, both within the assembled calorimeter and during construction. Of particular importance are local stresses, which may be design sensitive. In order to efficiently screen materials, some order of magnitude estimates were employed to provide a preliminary comparison of materials and to eliminate materials that would noi meet the system requirements. For instance, because creep properties were generally unavailable for the more complex alloys and composites, the creep strength was estimated to be about one half of the material yield stength at room temperature. Also, the load bearing requirement was estimated to be approximately 20,000 psi from several finite element model analyses of the barrel calorimeter. Although this value is actually design sensitive, it is important because it is much greater than the load levels that were expected on the basis of preliminary analyses.

The tensile properties of a wide range of lead alloys were collected and compiled (see Table I); a sumary is shown in Fig. 1.35. These tables clearly show that candidate lead alloys exhibit mechanical properties chat are below the $20,000 \mathrm{psi}$ requirement. The strongest of these alloys is $\mathrm{Pb}-0.065 \% \mathrm{Ca}-$ 1. $37 \mathrm{Sn}$, which exhibits a yield strength of about half of the acceptable value. Because it may be possible to use such an alloy in a lower stress 
region of the calorimeter, for example in the EMC section, creep rupture data were collected for this alloy and several similar ones as shown in fig. 1.36 This figure indicates that the creep rupture strength of $\mathrm{Pb}-0.065 \% \mathrm{Ca}-1.3 \%$ Sn for 1 ifetimes of greater than 11,000 hrs ( $=1.25$ years) is about 3000 psi. It is therefore concluded that lead alloys can only be considered for use in regions of the calorimeter where the computed stresses do not exceed $2500 \mathrm{psi}$, and that the alloy to be used should be $\mathrm{Pb}-0.065 \% \mathrm{Ca}-1.3 \% \mathrm{Sn}$. Concepts for using composite materials and techniques were included from the early stages of this study because lead alloys were known to exhibit poor mechanical properties by themselves. These concepts included: 1) cast alloy plates stacked with external supports. In this case, creep was anticipated to be a problem. 2) cast composite plates with stiffened outer plate layers. Both creep and fabrication problems were expected for this case. 3 ) a bonded structure with cast plates and the hot isostatic process (HIP) for bonding. An integrated structure would result from this concept, and perhaps a composite framework is possible, and 4) a cast structure with a composice framework and slots for installing the scintillator tiles. For these composite concepts, candidate mechanical systems were examined using a computer synthesis of the composite mechanical properties. In some cases, after determining mechanical suitability, laboratory castings were made to evaluate the viability of the composite and the difficulties of fabrication. Composite systems with the following reinforcements were considered:
1) Copper
2) Steel
3) Molybdenum
4) Tungsten

These reinforcing materials are ordered from the material that will provide least strengthening to the maximum strengthening. They are also ordered from 
the most easily "wetced" by lead, and thereby most easily processed into a composite, to the least easily wet by lead and least easily brocesid. The computer syntheses of the mechanical properties of the composites indicated that all of the materials could produce composites that would exhibit the required strength levels. Figure 1.37 compares the synthesized composite properties with the yield strength, ultimate tensile strength and elasticity of other candidate materials. It is also important to notice that, because the reinforcement bears most of the load in these composites, the mechanical properties of the composites will not be significantly time-dependent. Thus, the structural analysis can be based on short-time tensile data rather than on creep stength data.

Based upon the computer simulations, laboratory casting trials were conducted to determine the feasibility of fabricating lead based composites. In particular, the labcratory trials were performed to determine whether lead could be cast around reinforcements and whether the lead composite structures could be subsequently cast to shapes that would contain precision slots into which scintillator tiles could be placed (see Fig. 1.38 and 1.39). Although mechanical tests were not performed on these bars, their durability in handling indicated that they were much stronger than similar sized lead bars. In order to provide precision slots cast into the lead composites, a casting technique using a Teflon insert was developed. Figure 1.40 shows a casting that was made around a Teflon ingert, which, because of its high coefficient of thermal expansion and low coefficient of friction, was found to be very easily removed after the casting had cooled to room temperature. From the computer strength simulations and the laboracory casting trials, Lead based composites appear to be a viable materials option for a SSC calorimeter system. Because the data to date are only preliminary, several 
issues remain, such as the optimum casting geometry, the wetting of the reinforcement by the lead matrix, and the surface profuced by the teflon casting insert. These and other fabrication and manufacturing issues should be assessed and optimized. Nevertheless, it appears that composite lead based structures could be fabricated as EMC or HAC calorimeter modules. In these cases the reinforcement would be judiciously placed to absorb essentially all of the load, such as in Fig. 1.41. More appropriate and detailed concepts are currently under development.

Several suppliers of lead alloys and castings were contacted in order to support the development of cast lead composite structures. Communications with these vendors determined that large castings of the type proposed for the calorimeter modules were feasible and that these vendors (Fig. 1.42 ) would be available to provide this capability. Preliminary cost estimates were also explored with these vendors, and costs on the order of $\$ 1.50$ per pound were quoted for cast structures (Fig. 1.43). Thus, lead based composite concepts appear to be feasible based on mechanical and fabrication criceria, and also appear to be attractive based upon costs.

In order to fully develop these concepts for implenentation in a SSC calorimeter system, it is necessary to 1) verify the mechanical performance of the case composite structures, 2) determine the reliability of casting the composite structures, 3) assess the precision of casting scintillator slots using the Teflon-insert technique, and 4) optimize the casting process for fabricating the reinforcement and insert containing structures. The program proposed for the coming year to address these issues is summarized in Fig. 1.44. The program has been structured to develop casting technology and to optimize the structural elements using laboratory methods before developing prototype calorimeter module castings that will be evaluated in a Fermilab 


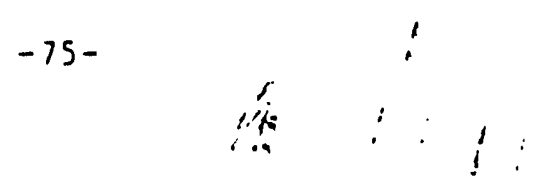

test beam. Figure 1.45 illustrates how the proposed program will lead to a phased development of technologies that are required for a léad bajsed calorimeter for the SSC. 


\section{FIGURE CAPTIONS}

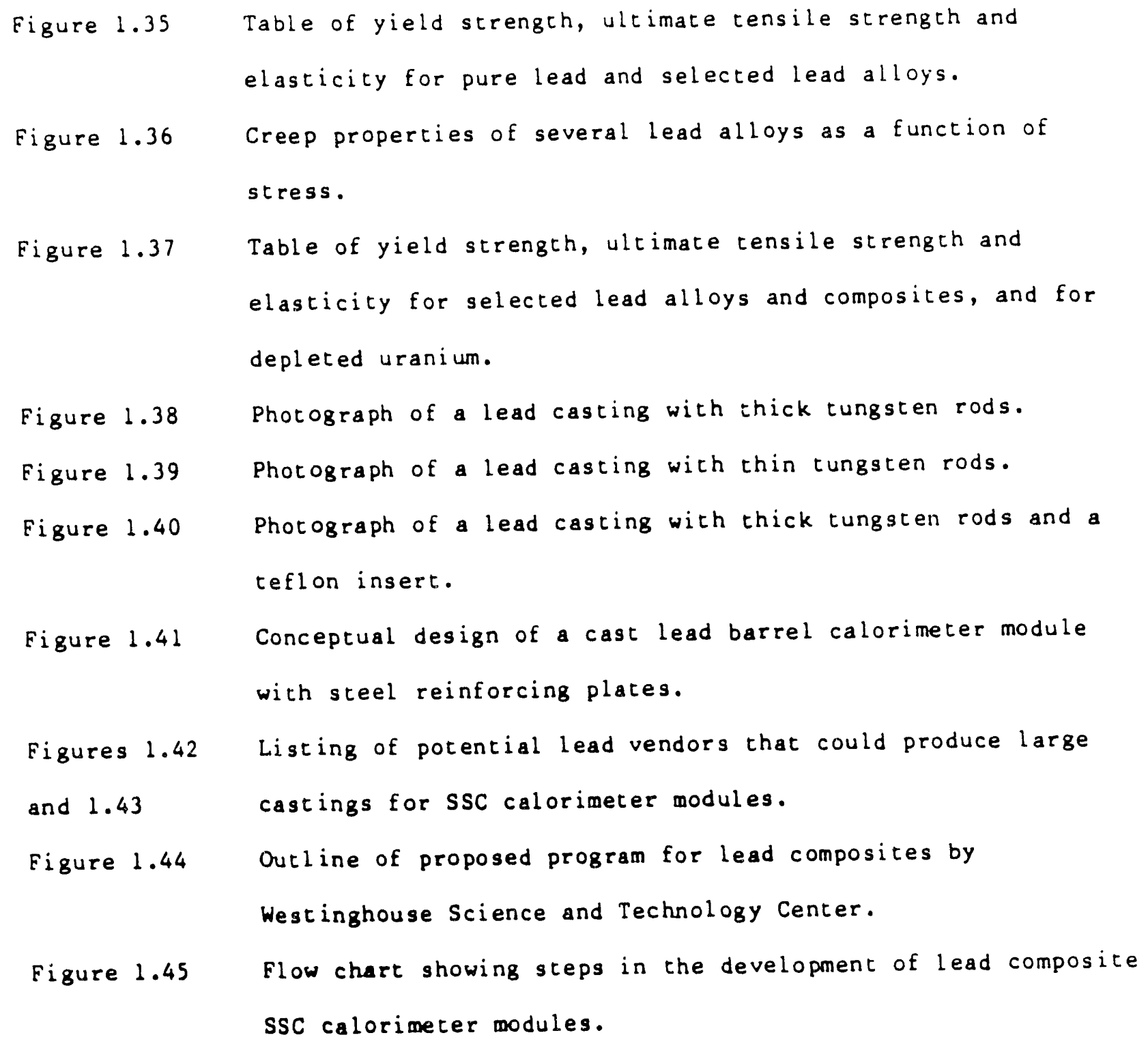




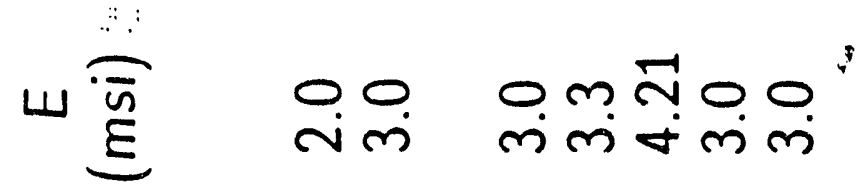

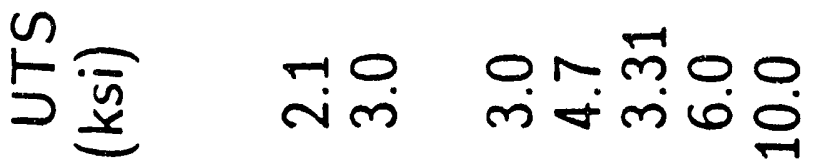

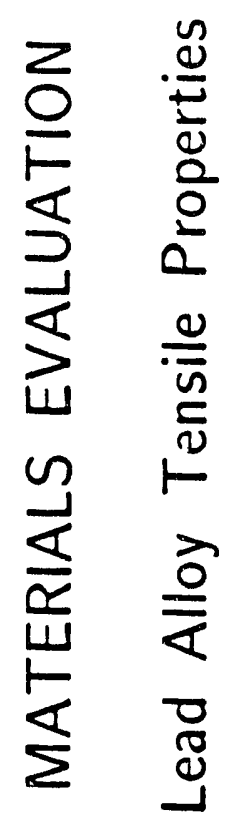

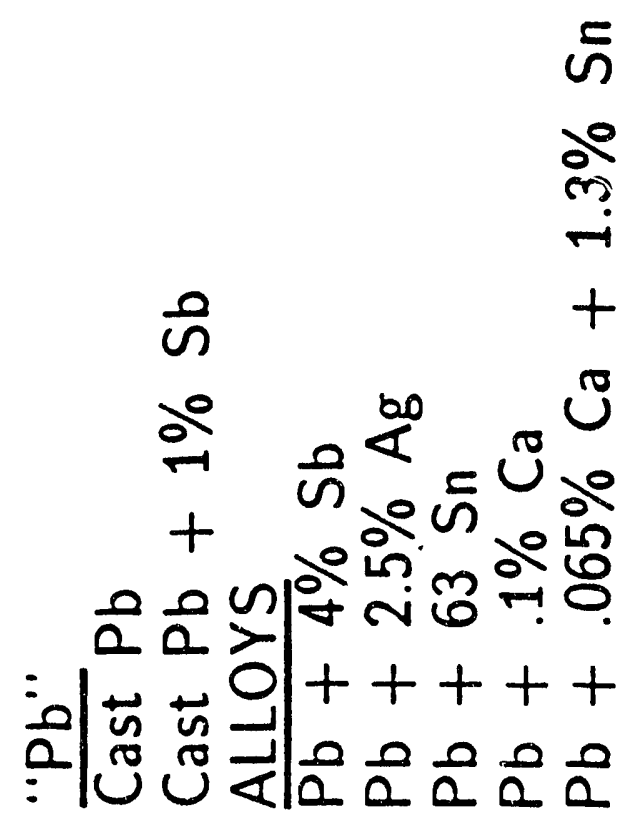

U 


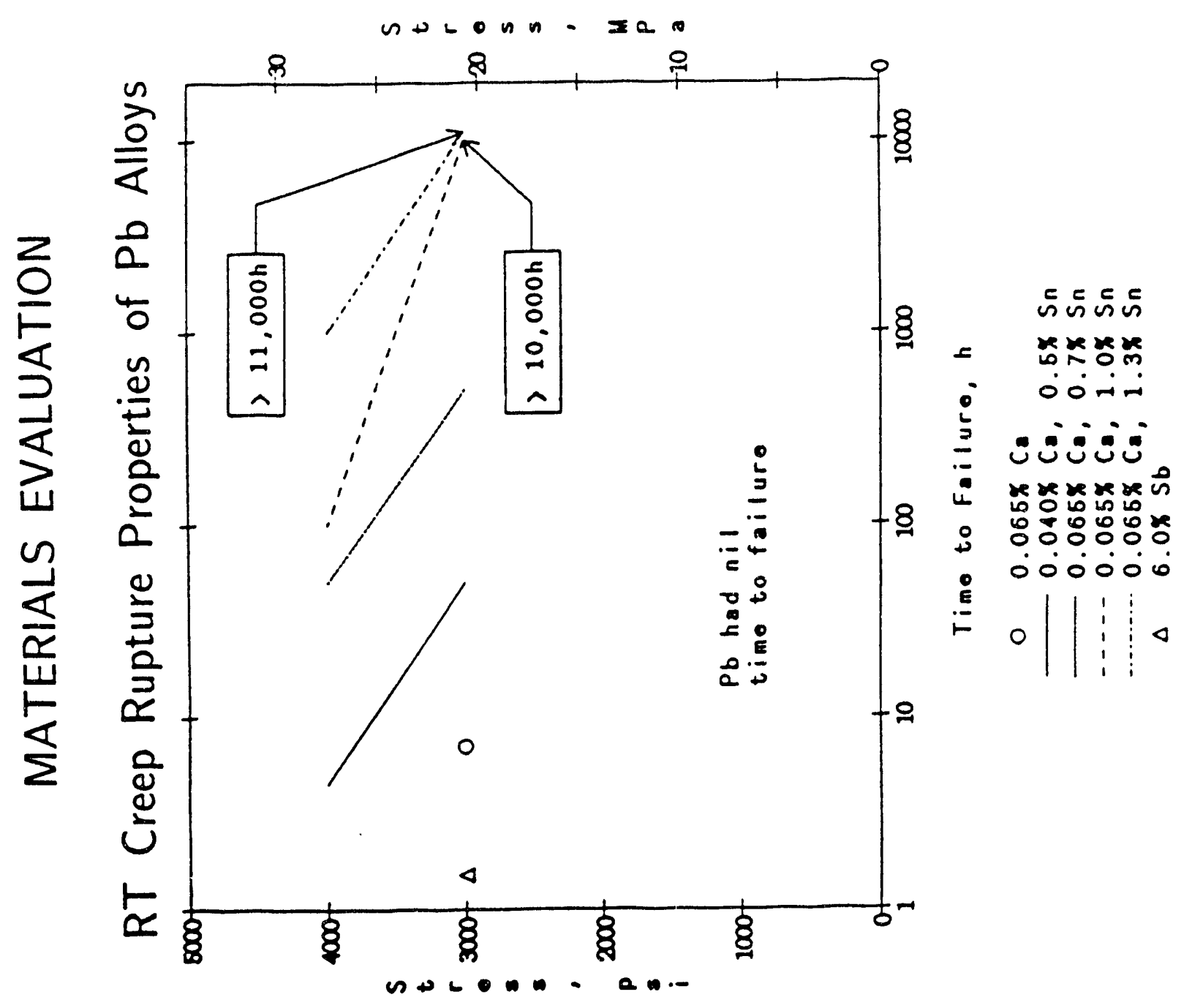




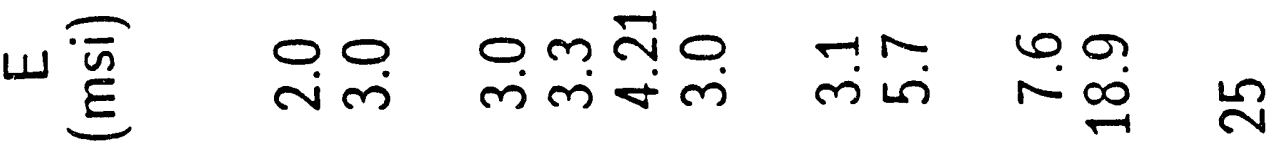

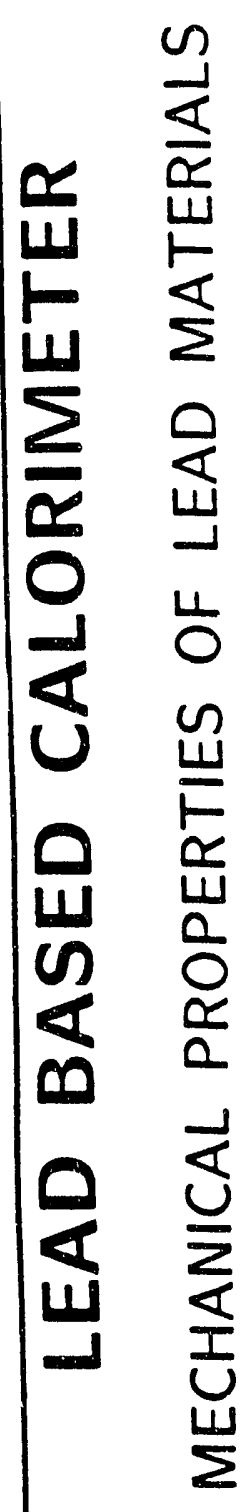

告恶

rim
m

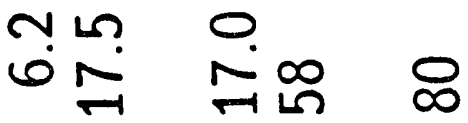

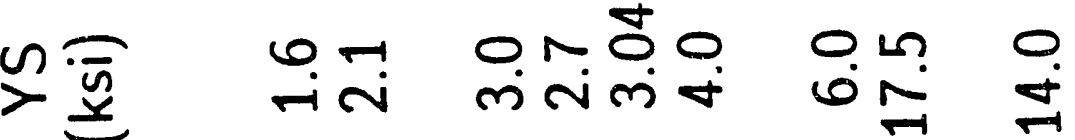

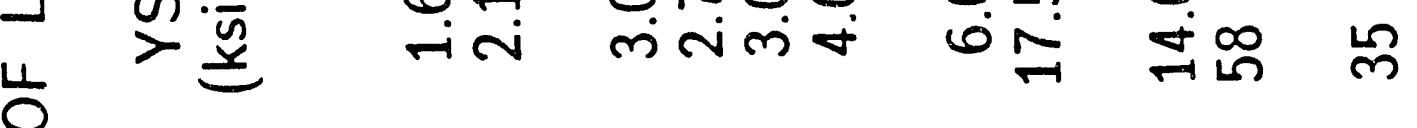

की

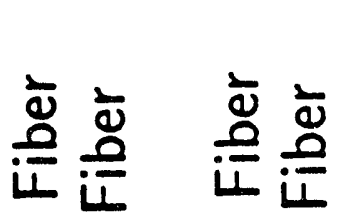

ำ

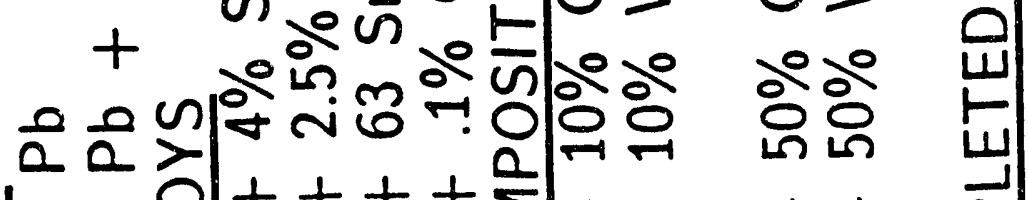

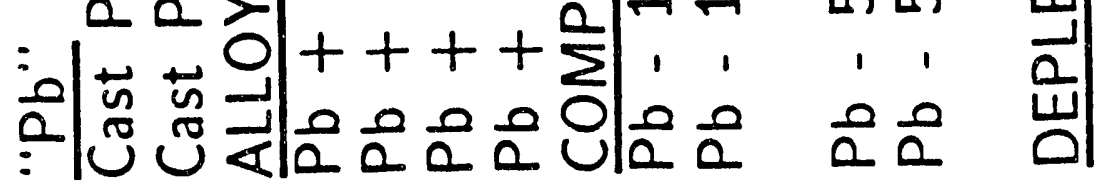




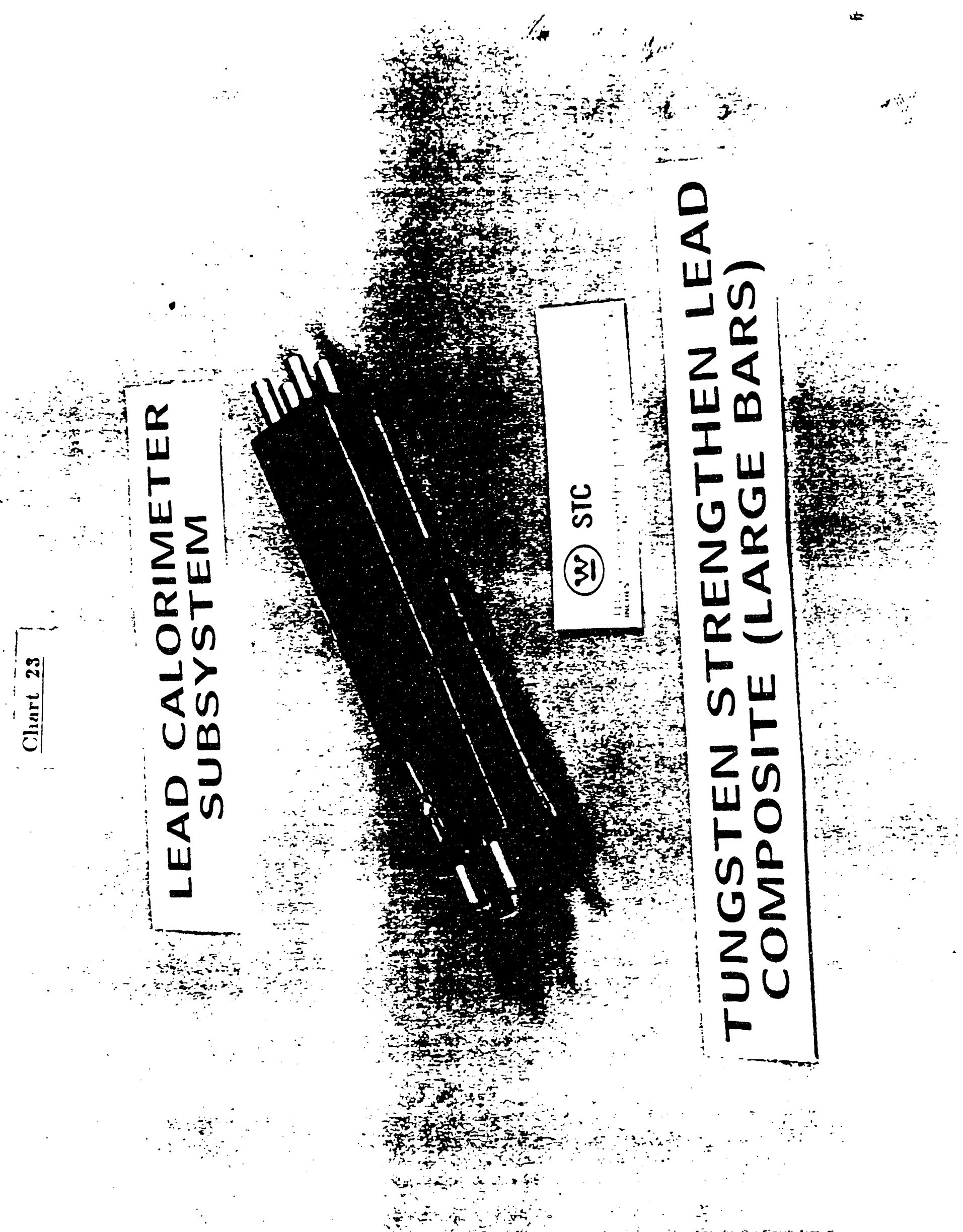




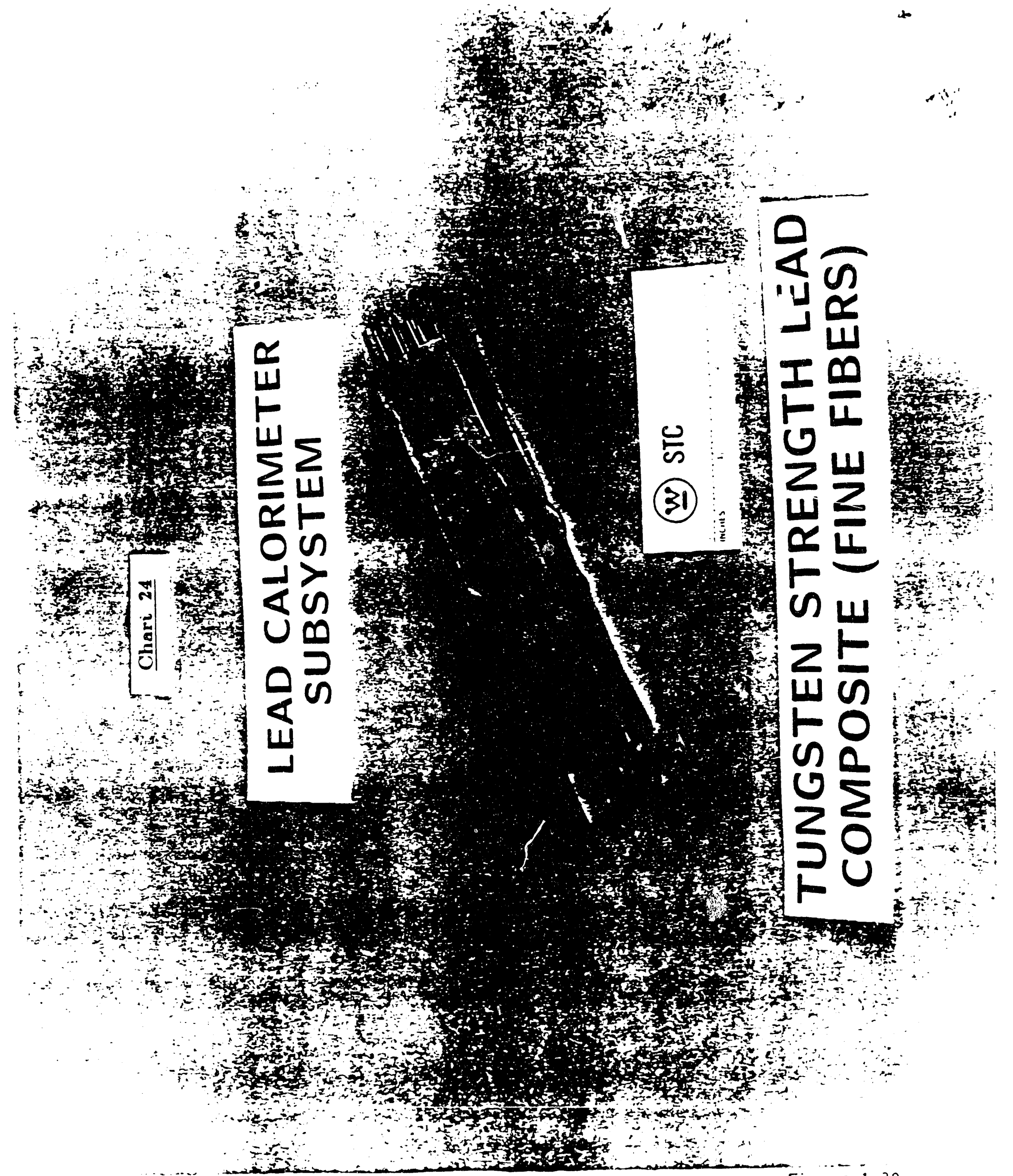




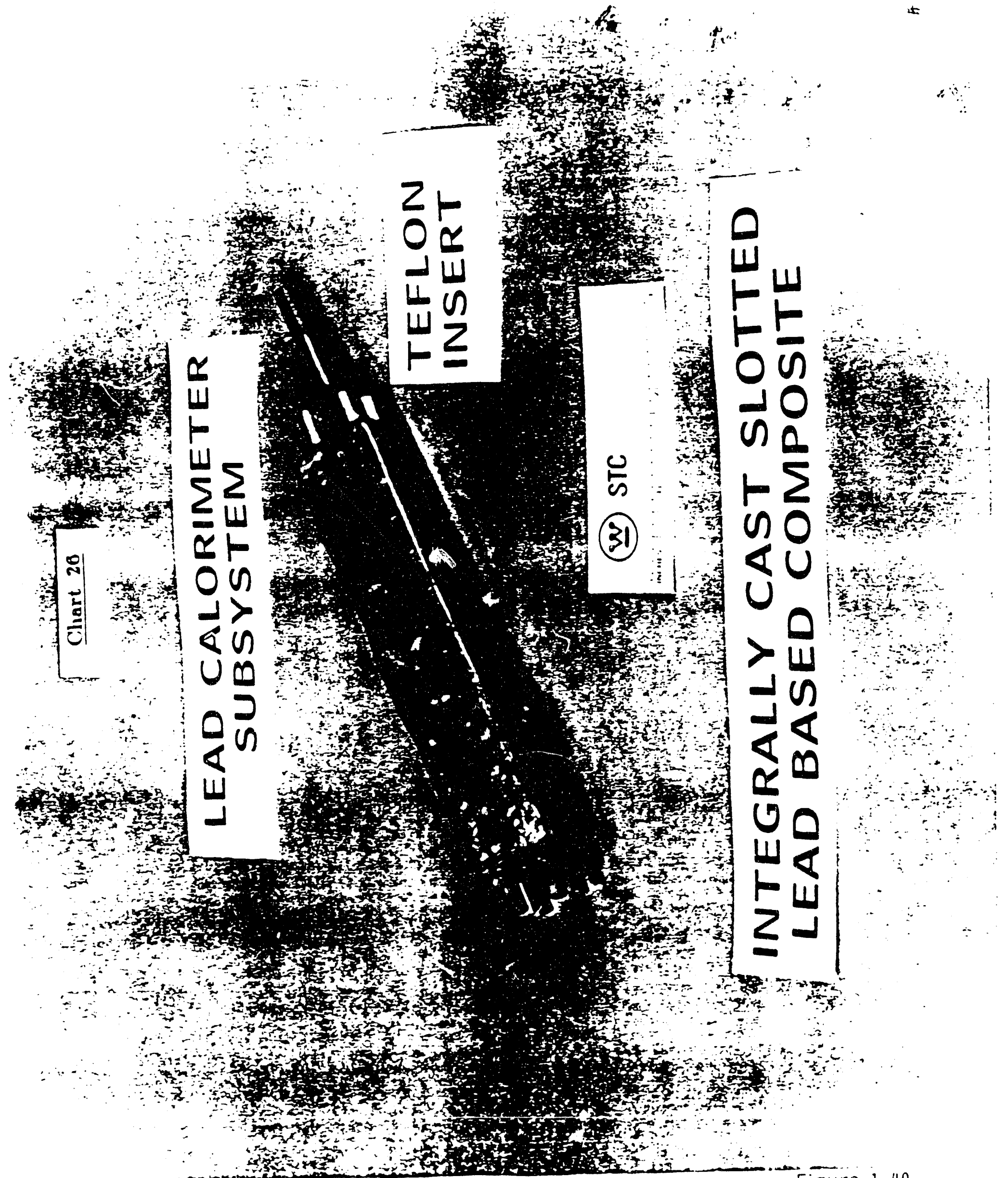

Figure 1.40 
$-83-$
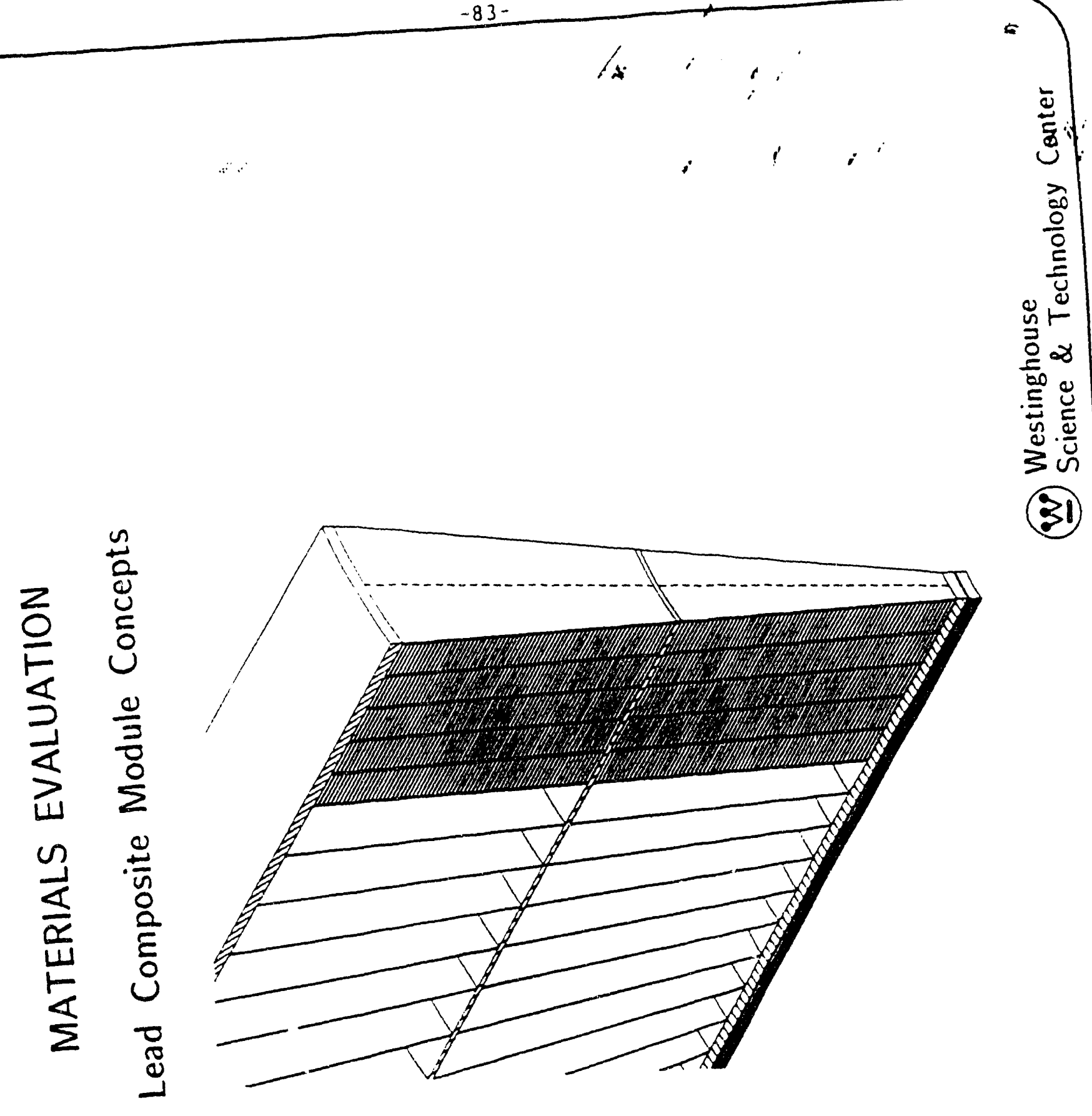

Figure 1.41 


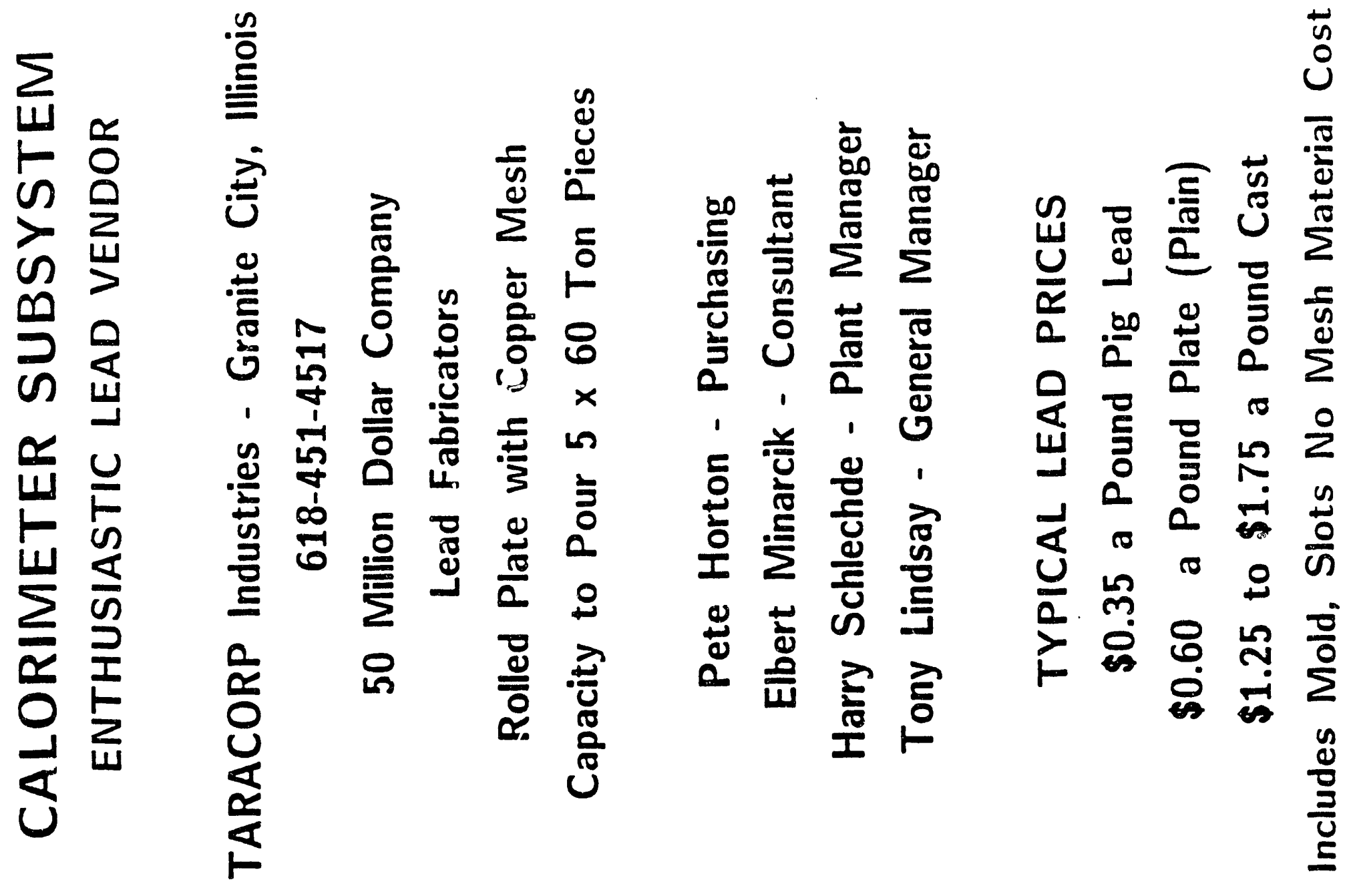



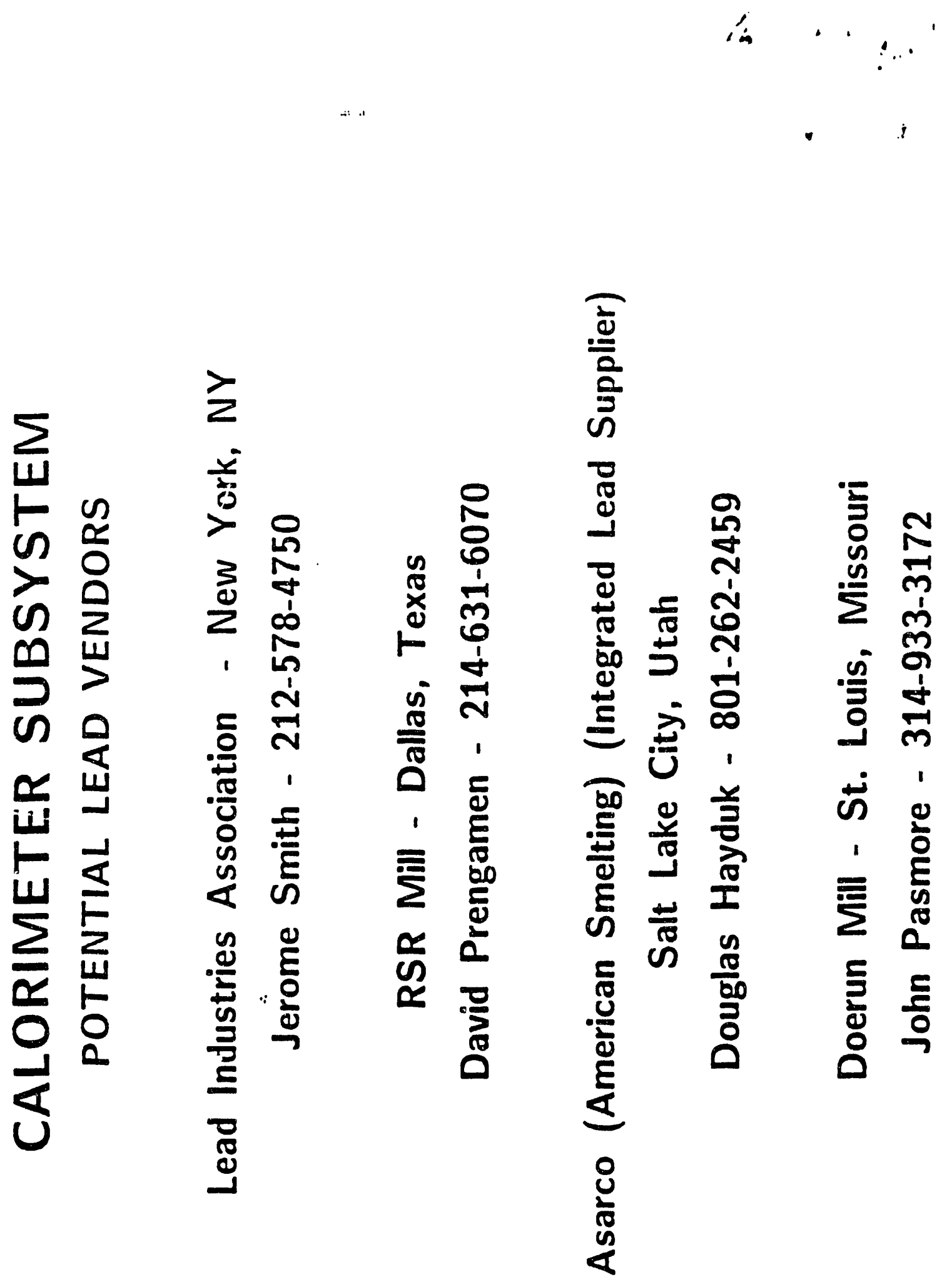


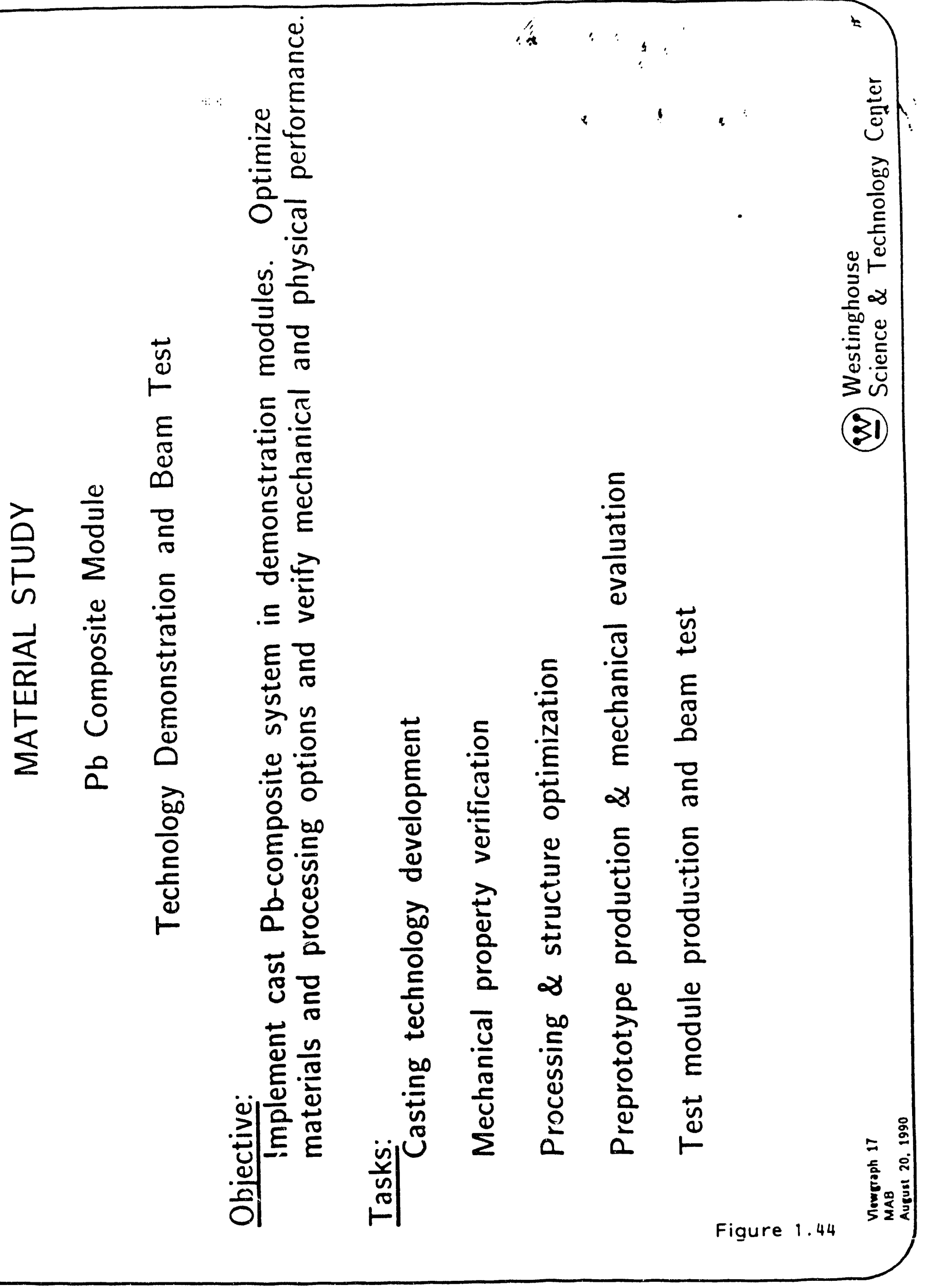




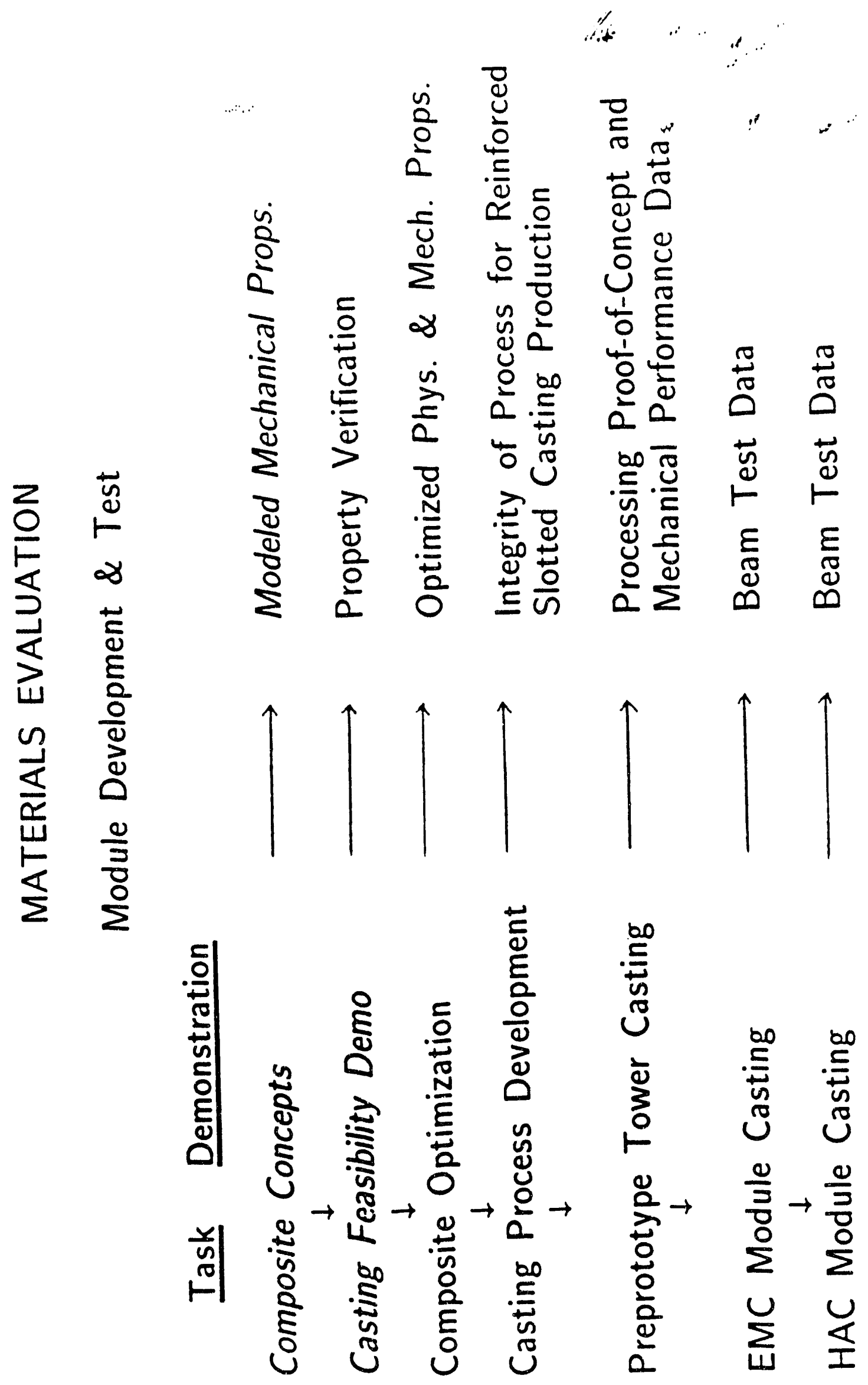


Summary of Wrought and Cast Lead and Lead Milloys' fór an; S'SC Calorimeter

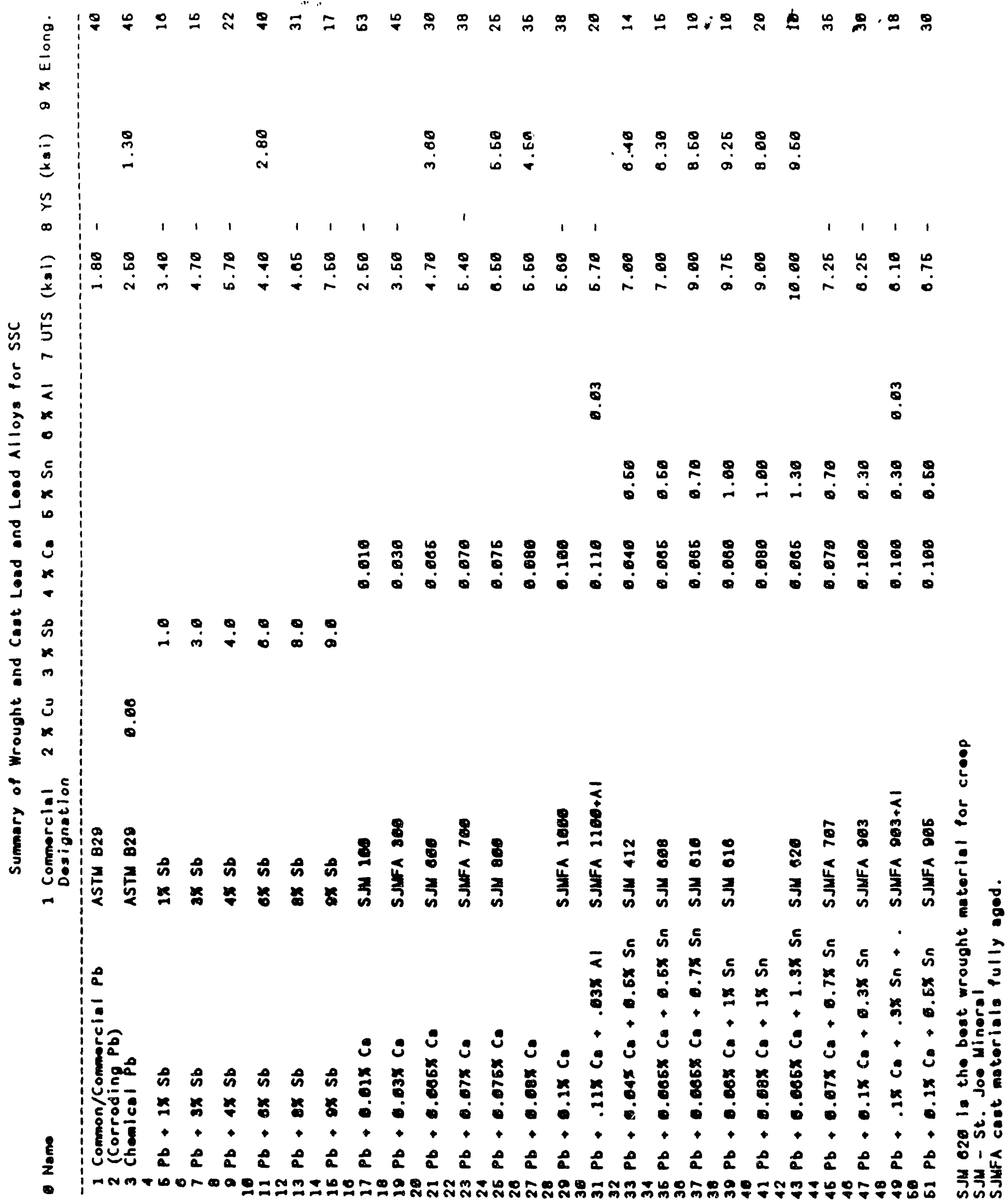




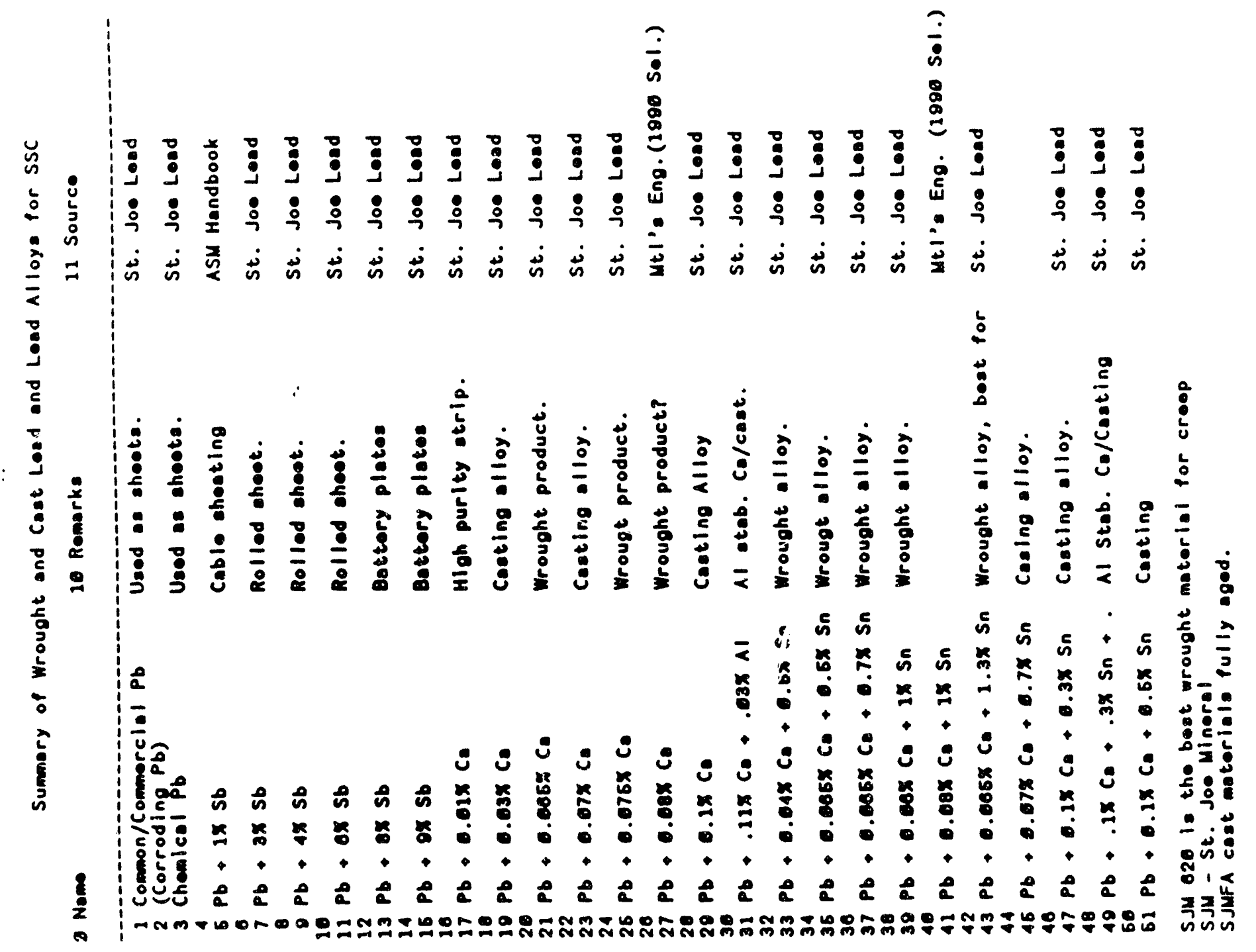




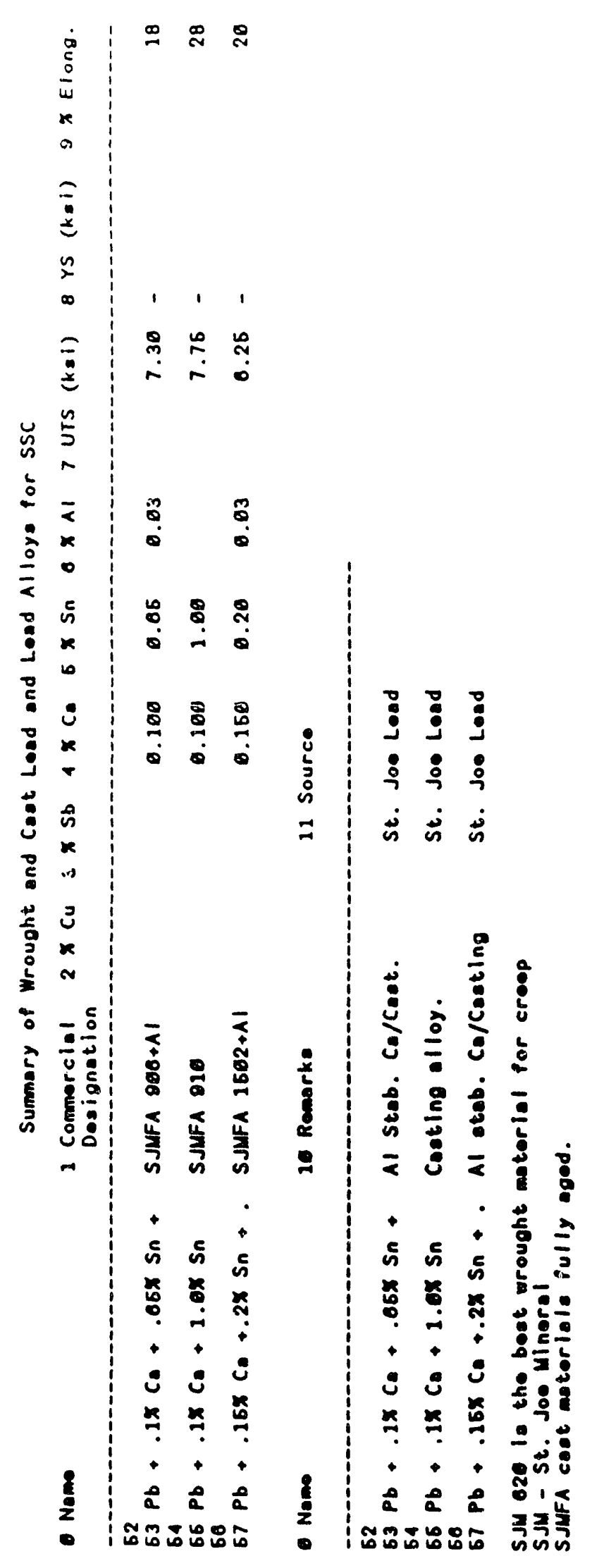




\section{SIMULATION STUDIES}

\section{P. K. Job, H. J. Trost, J. Proudfoot, L. E. Price "}

Three distinct categories of simulation study have been carried out:

a) benchmarking of the CALOR 89 code for two scintillator plate systems; b) a survey of the unit cell space for modest statistics; c) and initial CEANT simulations of the transition region between barrel and endcap calorimeters in the conceptual design.

a)

The CALOR simulation in this subsystem is by necessity distributed over several processors. As a result it was necessary to ensure that all codes were generating consistent results by having benchmark runs computed on each installation and a detailed comparison of results made (shower longitudinal and lateral profile, e/h etc.). The systems computed at Argonne are Fe/Scintillator and Pb/Scintillator. The energy deposition computed by each prccess is given in Table I. Further details of these runs are given in Appendix $I$.

Initial simulation studies of the unit cell (thickness and relative thickness of absorber and scintillator) have been carried out on the Cray and IBM computers at Argonne by P. K. Job. The objective is to determine the unit cell which optimiges individual electron and pion resolutions and simultaneously obtains a configuration in which both electrons and pions have equal response (e/h $=1$, or "Compensation"). The variation in the relative response of electrons to pions for depleted uranium (DU), lead and iron absorber systems as a function of absorber chickness for $0.25 \mathrm{~cm}$ scintillator is shown in Fig. 2.1. These results are in general agreement with the avalable experimental data and other simulation experiments (The 
ZEUS Detector, Status Report, 1989). Uranium and lead absorbers can both yield compersating systems whereas this is not the case"'tor purre iron absorbers. The time dependence of compensation is also an issue at the SSC due to the $16 \mathrm{nsec}$ bunch spacing as it is expected that some component is due to slow neutron capture. Preliminary results on this are shown in Fig. 2.2 and indicate that both depleted uranium and lead absorber systems yield approximate compensation $(e / \mathrm{a} \leq 1.05)$ in less than $16 \mathrm{nsec}$. Rapidicy dependence in e/h may be expected for sampling with constant absorber chickness. This has been studied for the compensating DU/scincillacor unit cell (Fig. 2.3) and we find $e / h \leq 1.05$ for $n<1.3$. The last in our series of initial studies was to evaluate the effect of varying the scintillator thickness for constant absorber thickness (Fig. 2.4). In this case we simulated this parameter in the vicinity of a $\mathrm{Pb} / \mathrm{scintillator}$ compensating geometry and observed the expected increase in $e / h$ with reduction in scintillation thickness.

To date these studies have been limited by availability of cpu and it is planned to extend them by approximately a factor of four in order that the limitation is in the computation model. To accomodate part of this we are presently commissioning the CALOR 89 code and geometry for scintillator calorimetry on the Lawrence Livermore Cray. These runs will be distributed amongst collaborators at Oak Ridge, University of Mississippi, University of Tennessee and Argonne. We anticipate a program studying a matix of four scintillacor thicknesses, four absorber thicknesses, three absorbers (lead, iron and depleted uranium) three particle energies and six integration gates to be completed in Fy9l.

c) A GEANT based simulation of support structure in the transition region between barrel and endcap calorimeter and the sulenoid coil has 
$-93-$

:."...

been initiated...to evaluate the benefit of a design with slightly nonprojective longitudinal geometry. This is obtained by requiring cowers in this region to project to a point in 2 which is offset from the true interaction point. The variation of response in the transition region as a Eunction of this offsec is shown in Fig. 2.5, indicating that an offset of approximately $40 \mathrm{~cm}$ may be adequate to cover this gap. However, many tests remair. to be done in FY9l and beyond (e.8. resolution, ratio of resolution to energy deposited, jet responses). 


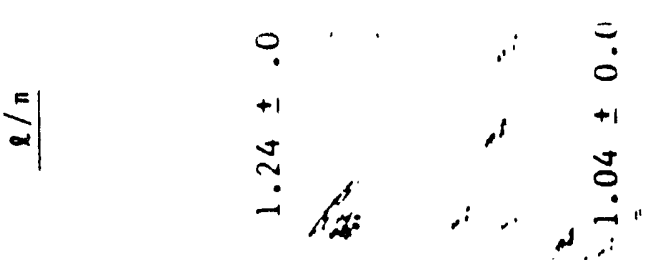

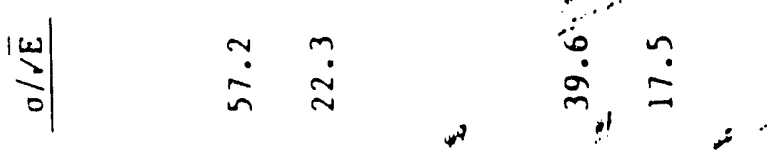

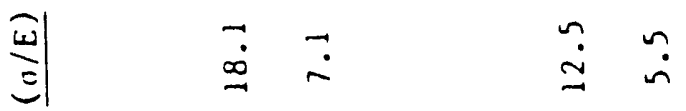

$\sum_{x}^{\infty} \mid \begin{array}{llll}\dot{0} & \dot{0} & \dot{\vec{j}} & \dot{n}\end{array}$

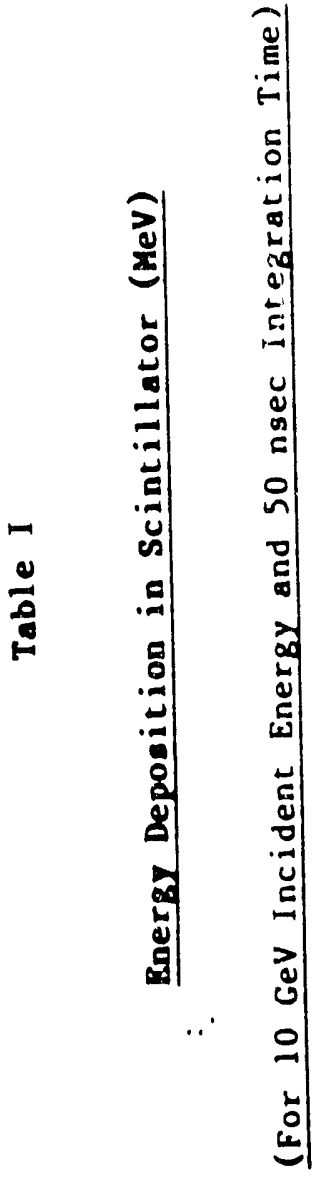

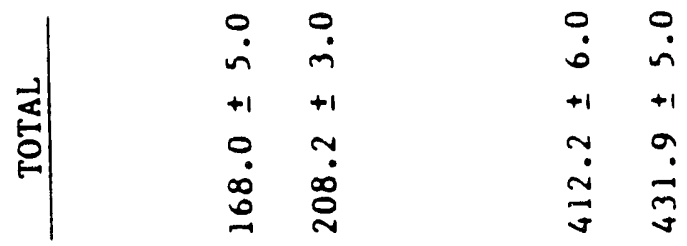

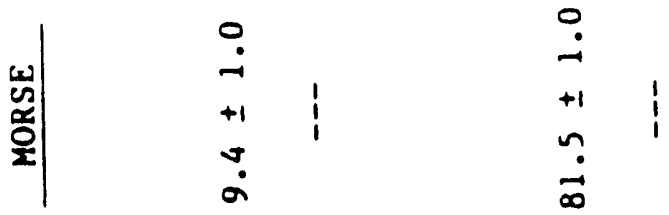

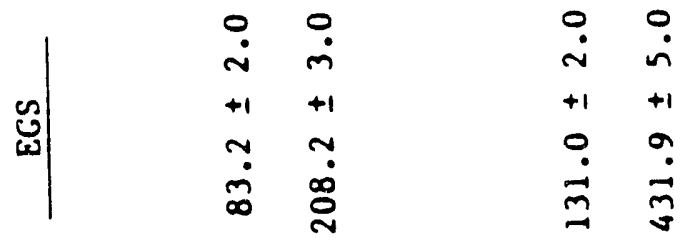

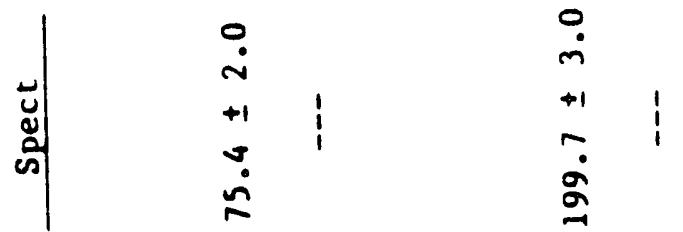

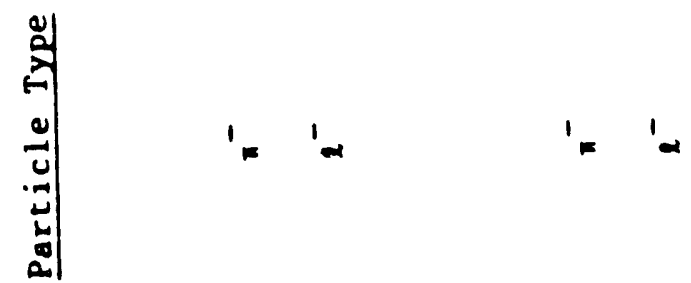

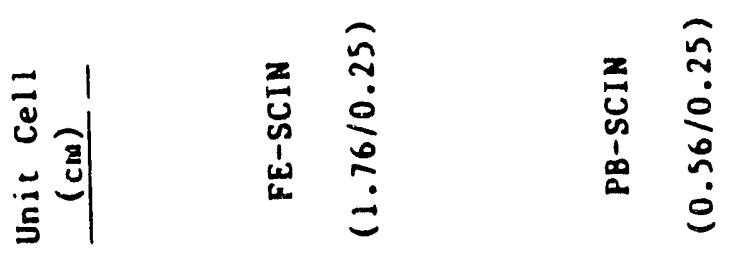




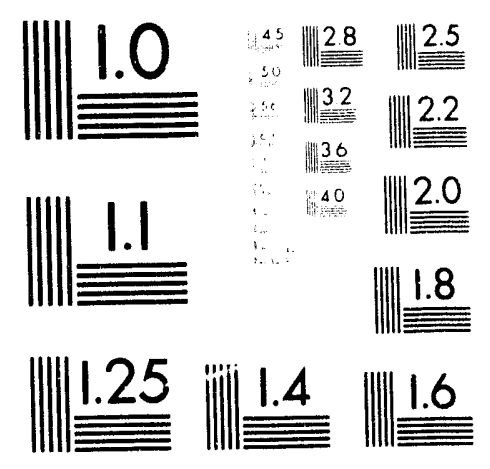



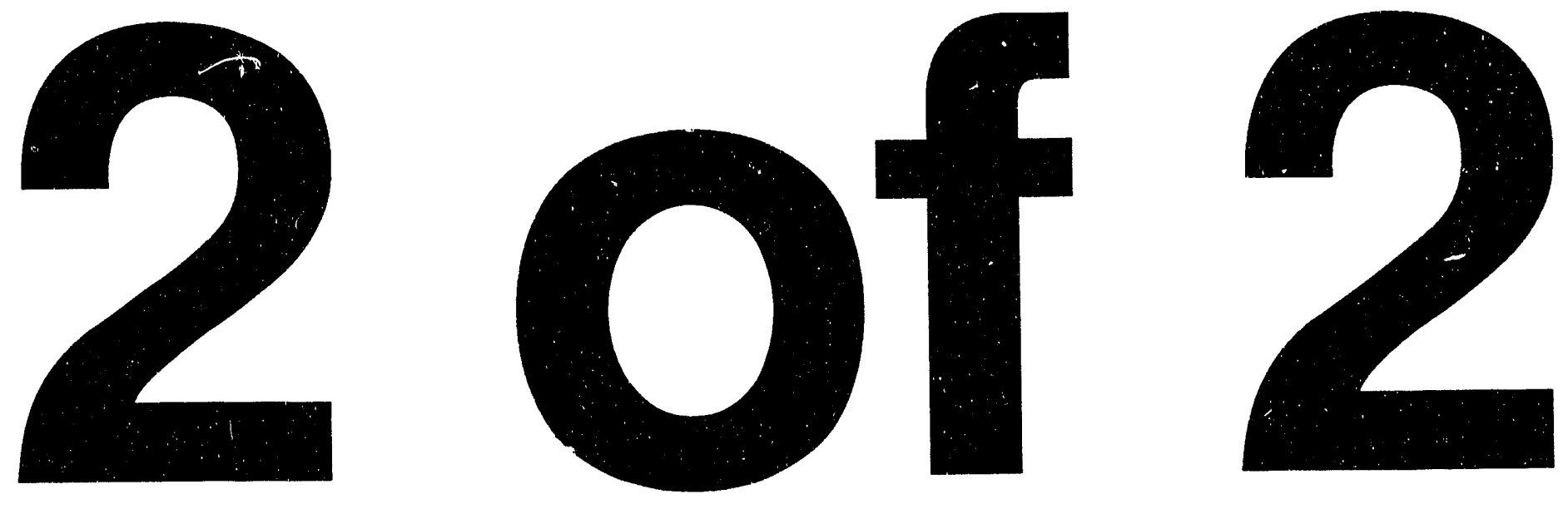


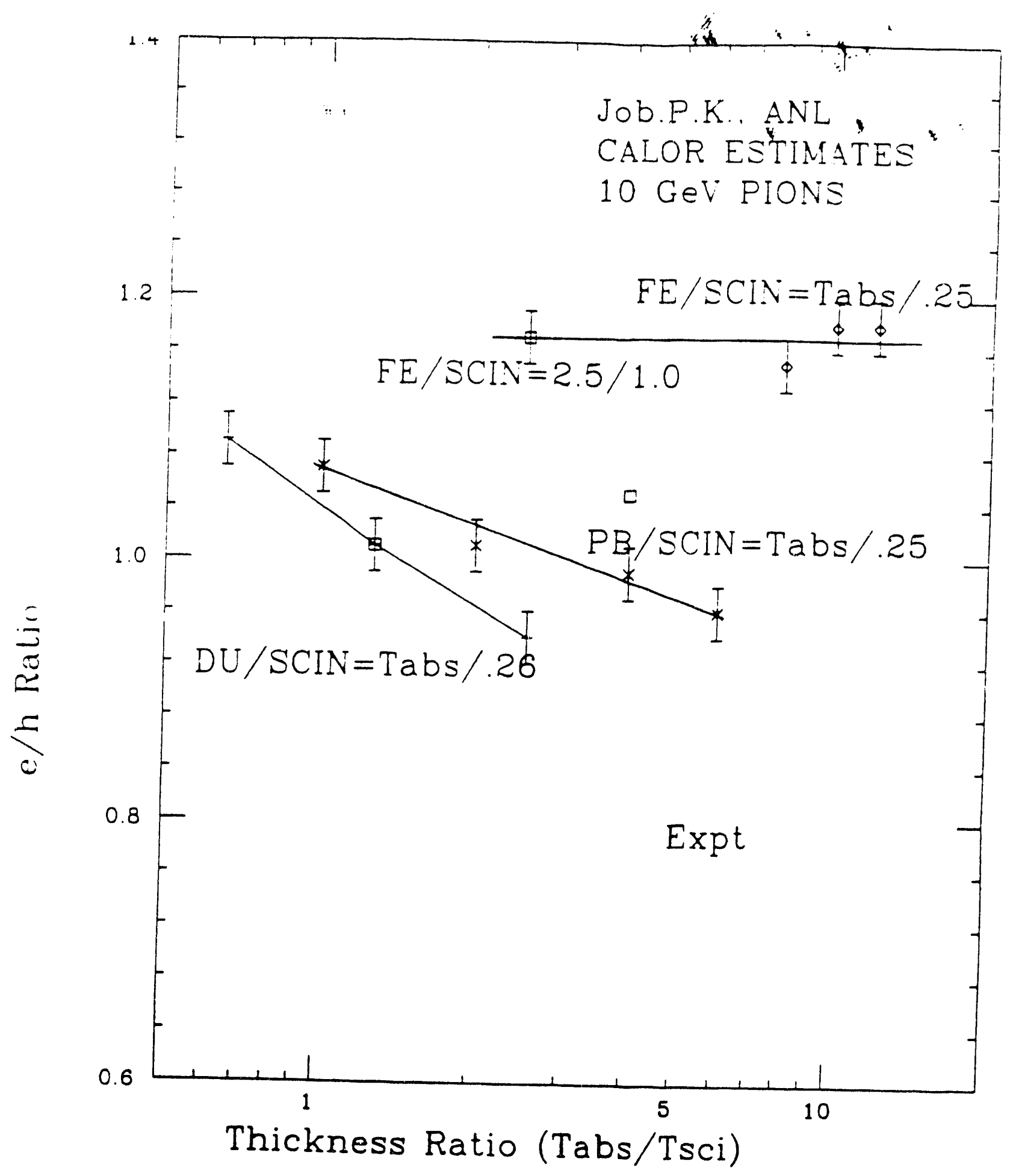

Relative response of electrons to pions (e/h) as a fuction of absorber thickness and composition for a fixed scintillator thickness of $0.25 \mathrm{~cm}$ Fig. 2.1 


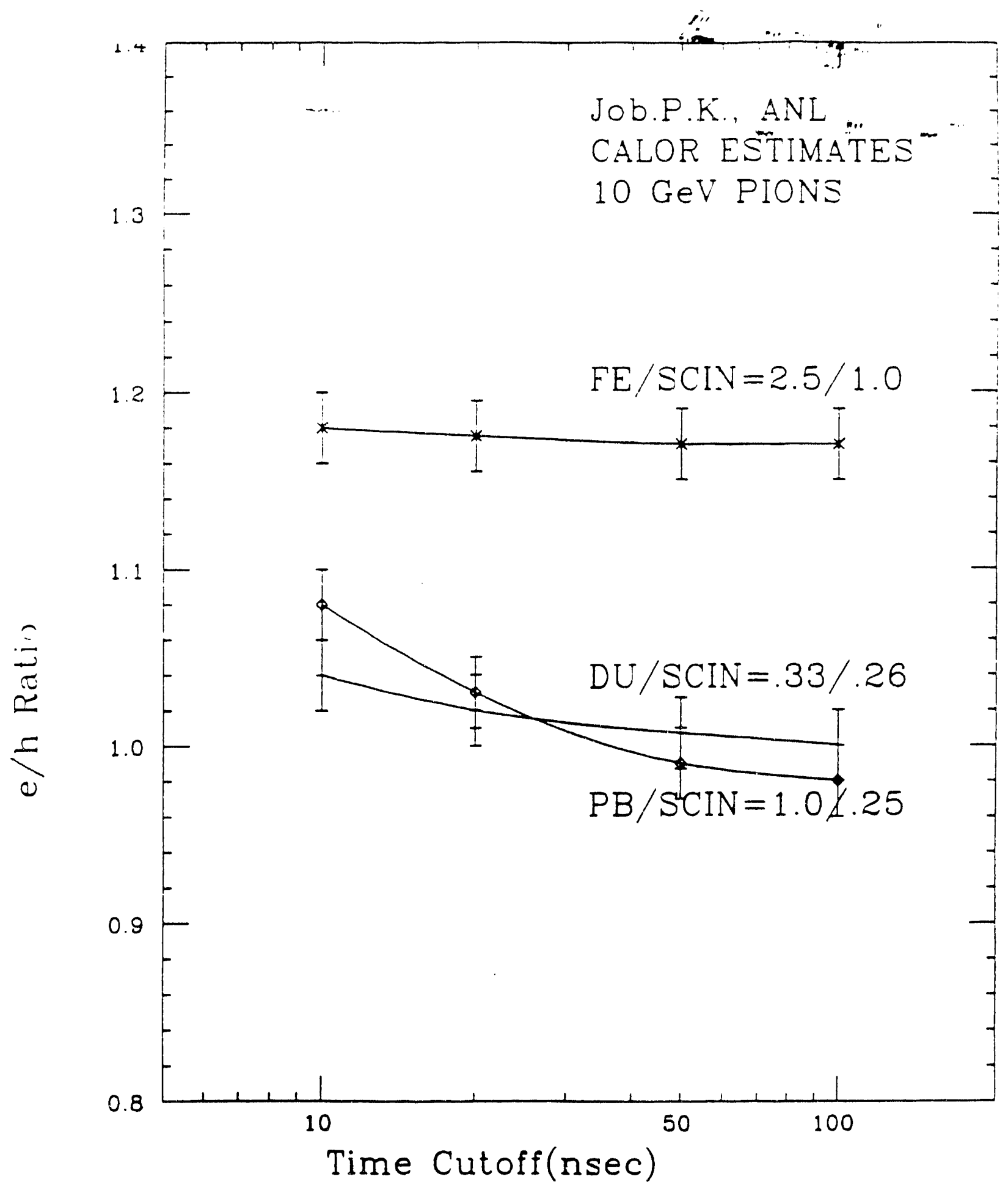

e/h as a function of integration gate for typical Iron, compensating Lead and Uranium geometries for Scintillator thickness of $0.25 \mathrm{~cm}$

Fig.2.2 
$-97-$

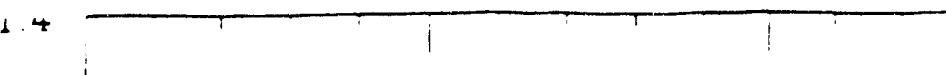

Job.P.K.A.VL

CALOR ESTIMATES

$10 \mathrm{GeV}$ PIONS

$\mathrm{DU} / \mathrm{SCIN}=.33 / 26$

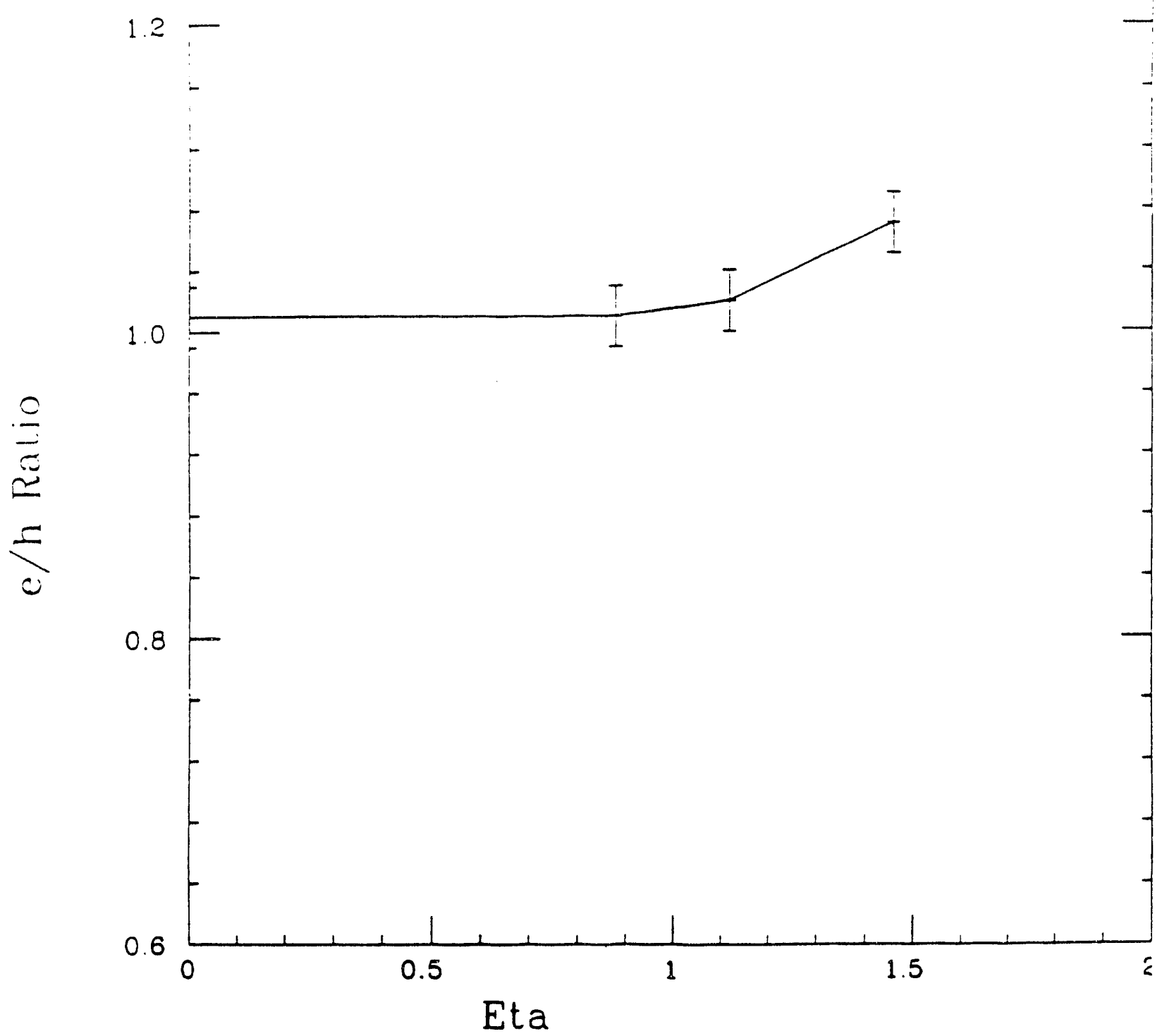

e/h as a function of pseudorapidity ( 11$)$ for a compensating Uranium geometry with Scintillator thickness of $0.26 \mathrm{~cm}$

Fig 2.3 


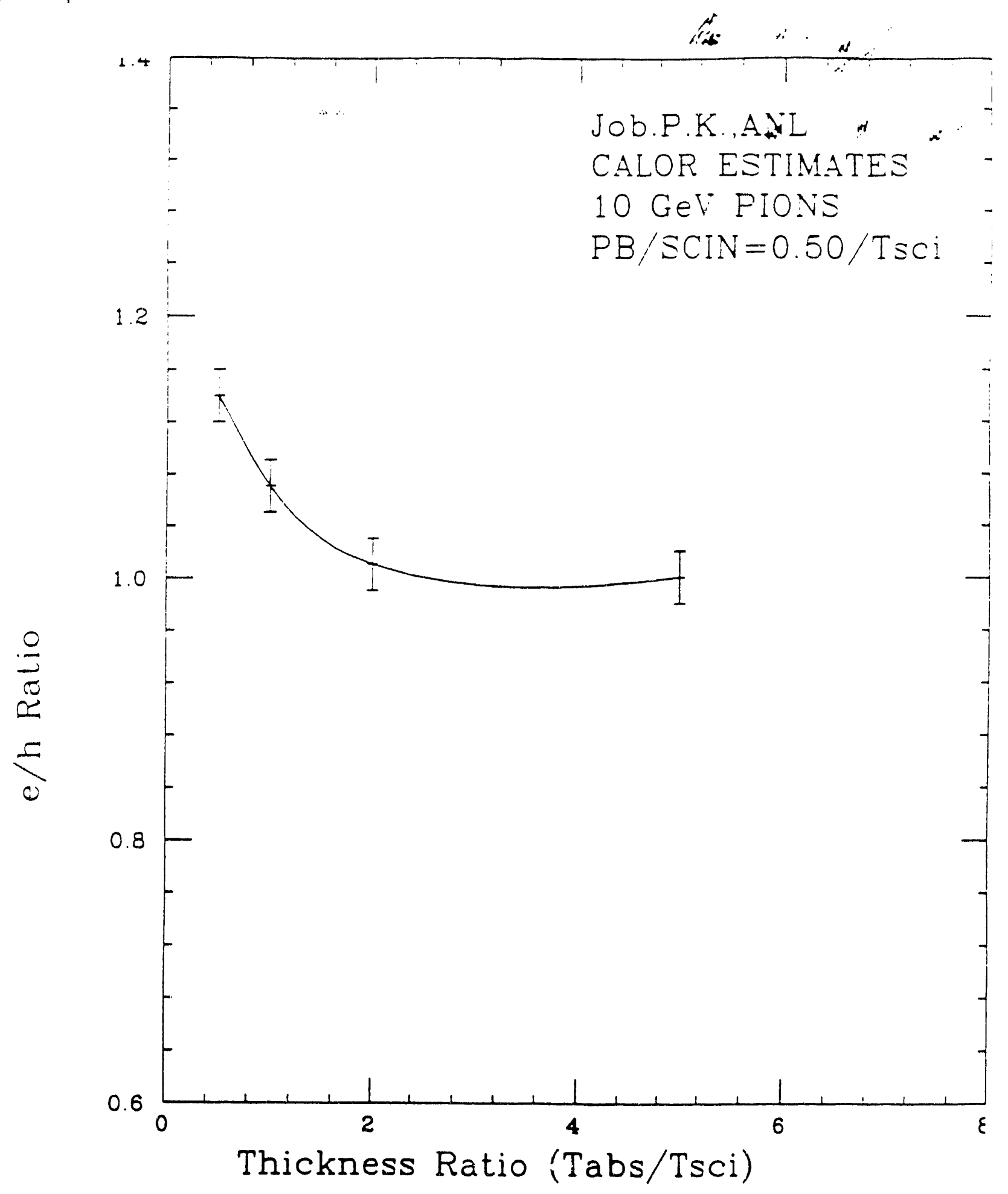

e/h as a fuction of Scintillator thickness for a fixed Lead absorber thickness of $0.5 \mathrm{~cm}$

Fig 2.4 
$-99-$

in
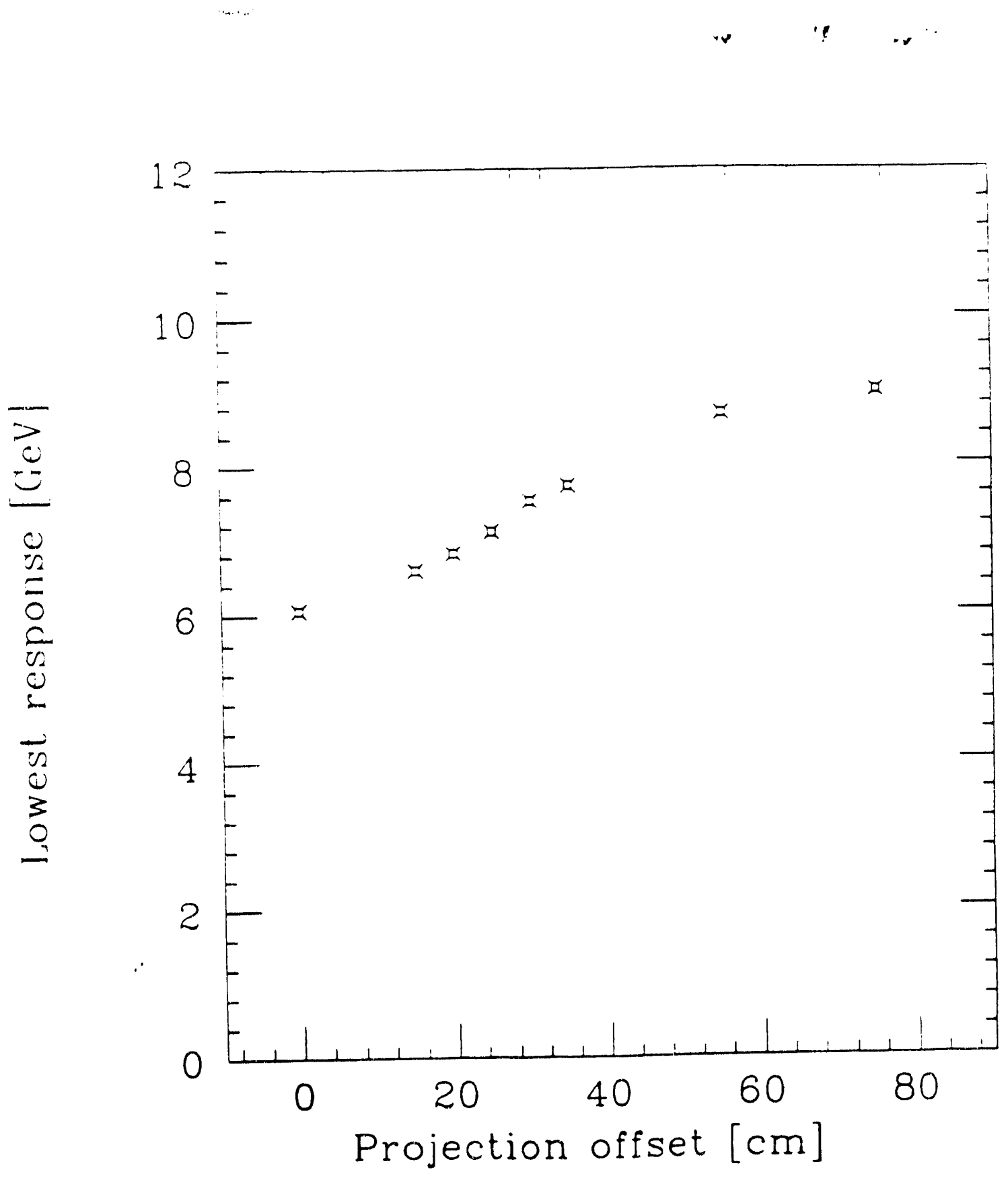

Minimal response in transition region

Figure 2.5 


\section{OPTICAL SYSTEX DESIGN}

D. C. Underwood, R. T. Hagstrom

Production of radiation hard scintillator plates by BICRON has been somewhat slower than hoped. This has been principally due to manufacturing difficulties in converting their RHI series from fiber to cast plate scintillator of appropriate mechanical and optical properties (as discussed in the section on the scintillator development program).

The first set of plates for testing were received in August. Initial tests included an evaluation of the mechanical variation in milling a groove, and an optical survey of the face of the plate to study surface crazing, performed by Ray Hagstrom. The preliminary conclusion is that although an acceptable quality of cut could be obtained, the plate showed considerable surface crazing, due to problems in casting, and would therefore be unlikely to form the basis of a high precision calorimeter. We are now in the process of resolving chis problem with BICRON.

While awaiting scintillator plates, work proceeded at Argonne to study particular aspects of the optical design. A stack compression fixture to be used in evaluating the mechanical properties of this new plastic has been designed and is in the process of being set up. Some critical tests have been carried out on the fabrication and uniformity of scintillator tiles read out by embedded wh fiber. In particular, a UV reflective foil capable of significantly improving the optical uniformity has been identified and a fixture for controlled bending and annealing of fibers into the grooves designed and fabricated (Fig. 3.1). This will be used in the near future to develop a radiation hard fabrication process. Finally a lead scintillation stack was 


$$
-101-
$$




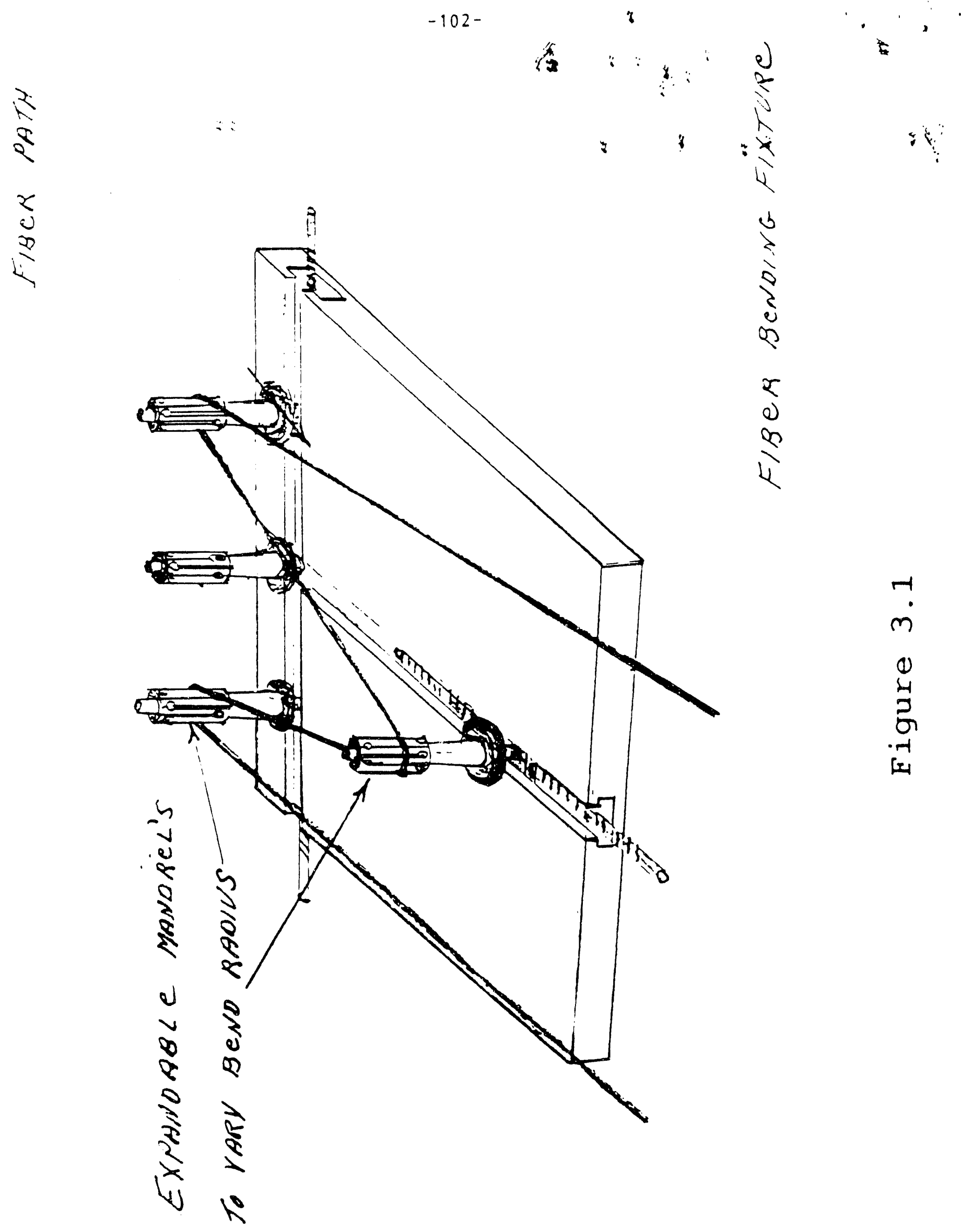




\section{Electronics Development}

C. Einolf, T. Porter, R. Ravas, and $S$. Weinstein

Science and Technoiogy Cencer

westinghouse Electric Corporation, Pitzsburgth, Pennsyluania 15235

j. Dawson, T. Ekenberg, D. Hall, J. Schlereth, A. itevens, and R. Talaga

High Energy Physics Division

Argonne Nacional Laboratory, Argonne, Illinois 60439

The electronics development tasks included gathering information on radiation hard processes for producing electronic chips, obcaining data on existing fast analog to digital ( $A / D$ ) converters, evaluating methods of locating the calorimeter electronics remotely, and preparing plans for electronics for a test beam module. As was anticipated, it was found that much of the electronics development for other subsystems was inappropriate for the fast signals with a large dynamic range from a scincillator calorimeter. Nevertheless, in order to prevent duplication of effort, considerable time was spent understanding and coordinating activities with groups working on electronics in other subsystems.

In a survey of radiation hard processes, daca were collected on chips produced by UTHC (aMOS - Fig. 4.1), AT\&T (Bipolar), Tektronix (Bipolar - Fig. 4.2), and Westinghouse (BICMOS - Fig. 4.3). It was concluded that existing processes would probably be adequate for electronics mounted on the outside of the barrel calorimeter, especially with the revised estimates of radiation Levels by $D$. Groom et al.

In discussions with personnel at Westinghouse ATL in Baltimore, it was suggested that developments for high detinition television (HDTV) would probably result in cheap A/D converters coupled with memory lookup that could correct for nonlinearities and potentially perform conversion of fulse height 
to energy (see Fig. 4.4). The development timescale for such devices is the rext Eew years. This will probabiy occur betore a tinal dechsion in" electronic components for an SSC calorimeter would be required.

Cons:cerabie effort was devoted to eraluating the possibility ot iocating the electronics remotely, away from the calorimeter. Advantages would include accessibility, ease of maintenance, removal of electronics from siznificant radiation doses, reduced problems with cooling, and porencial ease of upgrading the electronics. The systems considered involved eitner using fider optics for signal transmission berween phocodecectors on the calorimeter and remote electronics, or bringing fibers from the sintillator tiles to remote photodetectors and electronics. A "fallback" scheme using coaxial cables between the photodetectors and the electronics is also under consideration.

An investigation of the performance and cost of fiber optic links for the cransmission of data from the outside surface of the calorimeter was begun. Both analog and digital fiber optic link systems (transmicter, fiber optic cable, receiver) are available. Analog bandwidths above $200 \mathrm{MHz}$ and digital rates up to 2 Gigabits/sec exist for these systems anc sypical costs per channel are $\$ 300$ and above. A very approximate cost estimate is provided in Fig. 4.5 assuming 10,500 cowers with EMC, HACI and HACII sections each. Long, Low atcenuation fibers are required for the approach of bringing l'ght from the scintillator tiles ts remote photodetectors and electronics. For example, if the electronics were to reside on the outside of the outer muon chambers at a radius of - lom from the beamline, then a minimum length of the optical fiber would be $8 \mathrm{~m}$. Low loss glass and clear acrylic fiber have been consijared for this purpose. The much higher cost of glass Eiber $(\$ 3000 / \mathrm{km}$ vs. $\$ 200 / \mathrm{km}$ for acrylic fiber) and potential problems with bonding the glass fibers to the whs fibers embedded in the ecintillacor tiles have focussed work on the acrylic fibers for this application. Note that the total 


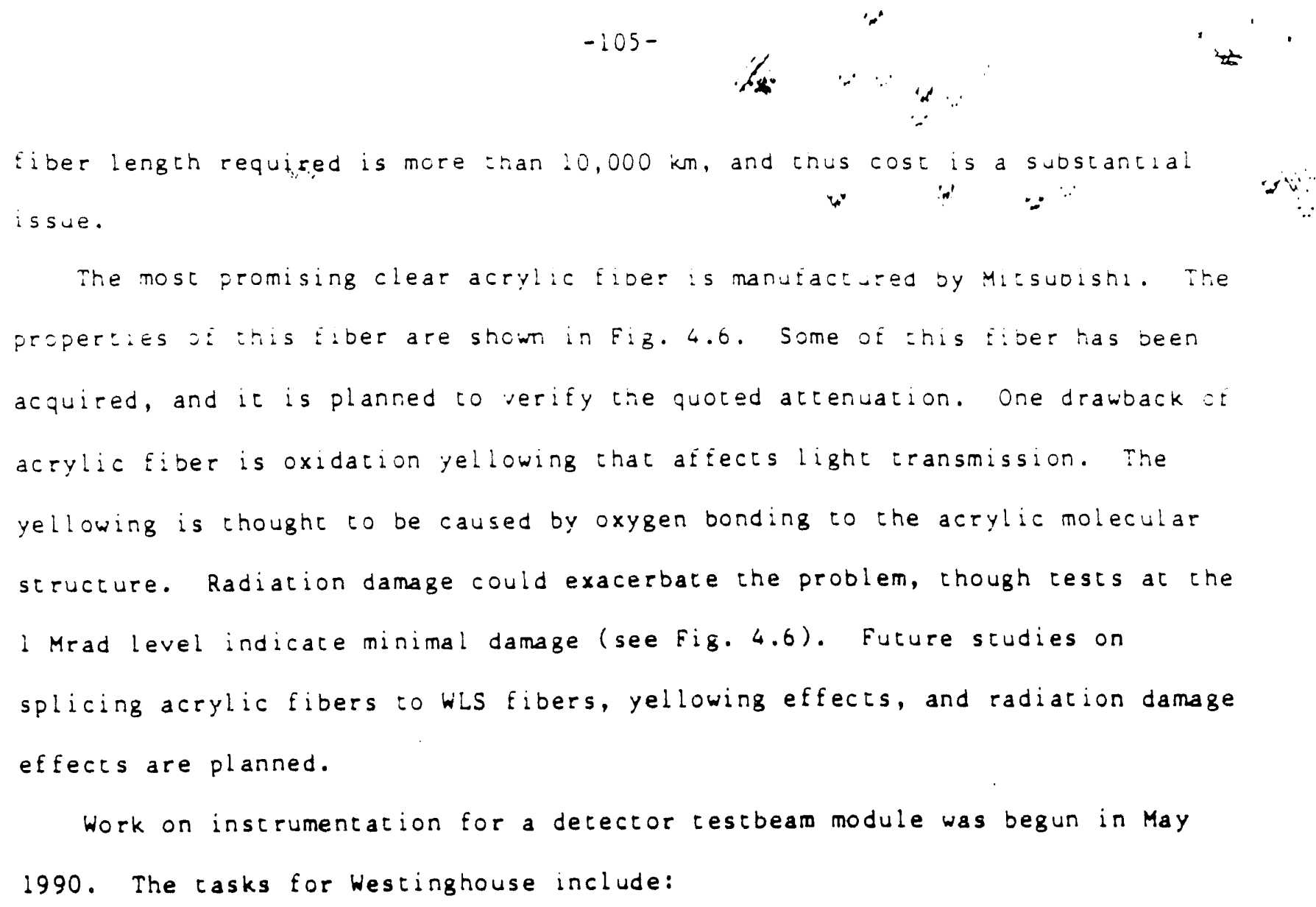

The most promising clear acrylic tioer is manutacrored by Mitsuolshi. Ihe properties j: ihis Elber are shown in Fig. 4.6. Some of zhis itoer has been acquired, and it is planned to rerify cre quoted atcenuation. One drawback ot acrylic Eiber is oxidation yellowing that affects light transmission. The yellowing is thought to be caused by oxygen bonding to che acrylic molecular structure. Radiation damage could exacerbate the problem, chough tests at the 1 Mrad level indicate minimal damage (see Fig. 4.6). Future studies on splicing acrylic fibers to whs fibers, yellowing effects, and radiation damage effects are planned.

Work on instrumentation for a detector testbeam module was begun in May 1990. The rasks for Westinghouse include:

1) Complete the design of an analog storage module urilizing the LBL integrated circuit design of $\mathrm{S}$. Kleinfelder et al. Produce a layout of a PC board for the module including shaping, capacitive storage, buffer and multiplexing functions.

2) Fabricate, rest, and debug a prototype analog storage module and .. assist in tests of its performance on a testbeam calorimeter module.

The tasks for Argonne include:

1) Design a VLI chip to store analog signals from a scincillator plate calorimeter. The photomultiplier signals are integraced for l6 nsec and stored on capacitors. Integration, storage, and digitization are performed or. single chip upon issue of a second level trigger signal. The architecture stresses simplicity of logic and has the potential for deadtimeless operation. The VLSI chip design has been begun (see Figs. $4.7-4.11$ ), and it will be subnitted to che MOSIS foundry by the beginning of November 1990. The chip is expected back 


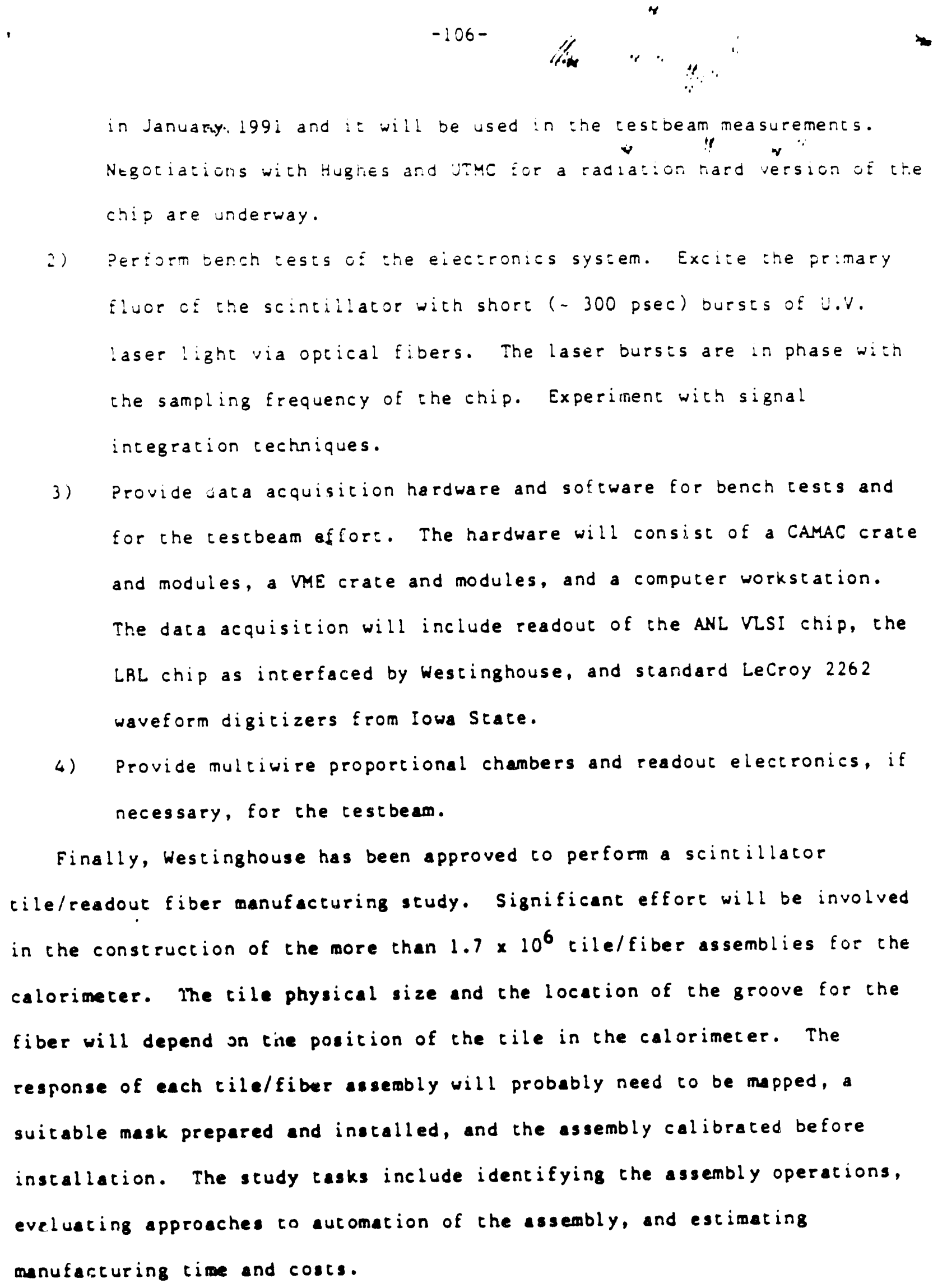

Finally, Westinghouse has been approved to perform a scintillator tile/readout fiber manufacturing study. Significant effort will be involved in the construction of the more than $1.7 \times 10^{6}$ tile/fiber assemblies for the calorimeter. The tile physical size and the location of the groove for the fiber will depend on the position of the tile in the calorimeter. The response of each tile/fiber assembly will probably need to be mapped, a suitable mask prepared and installed, and the assembly calibrated before installation. The study tasks include identifying the assembly operations, eveluating approaches to automation of the assembly, and estimating manufarturing time and costs. 


\section{FICURE CAPTIONS}

Eigure 4.: Intormation Erom UTMC on their radiation hard manufacturing

process for electronic chips.

Figure 4.2 Information trom Tektronix on their radiation hard

manufacturing process for electronic chips.

Figure 4.3 Information Erom Westinghouse A.T.L. on cheir radiation hard manufacturing process for electronic chips.

Eigure 4.4 Possible use of Euture high definition relevision (HDTV) analog to digital (A/D) converters for the calorimeter readout.

Figure 4.5 A very preliminary cost estimate for locating the electronics remotely, or far from the outside surface of the calorimeter.

Figure 4.6 Properties of Mitsubishi clear acrylic optical Eibers.

Figure 4.7 Schematic layout of the ANL VLSI chip.

Eigure 4.8 Schematic diagram of the input circuit for the ANL VLSI chip. Three amplifiers are used to l) incegrace the photodecector current, 2) store the corresponding voltage in analog memory, and 3) reset the memory cell.

Figure 4.9 Switch settings for the input amplifiers on the ANL VLSI chip.

Eigure 4.10 Schematic diagran of one of che eight capacitor arrays on the ANL VLSI chip.

Figure 4.11 Schematic diagram of the output circuit on the ANL VLSI chip, showing digitization of the analog signal on receipt of a level-2 trigger "ACCEPT" signal. 


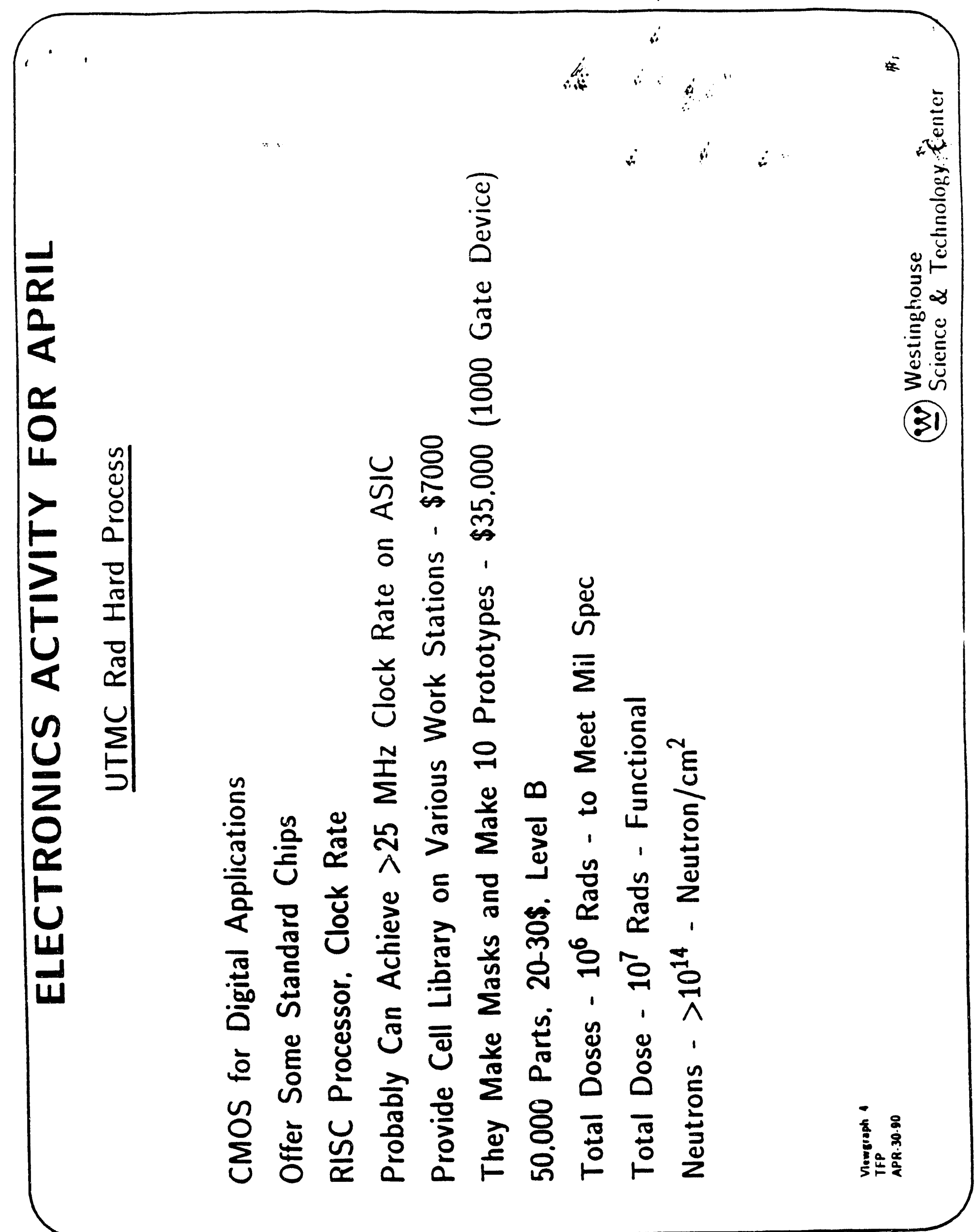




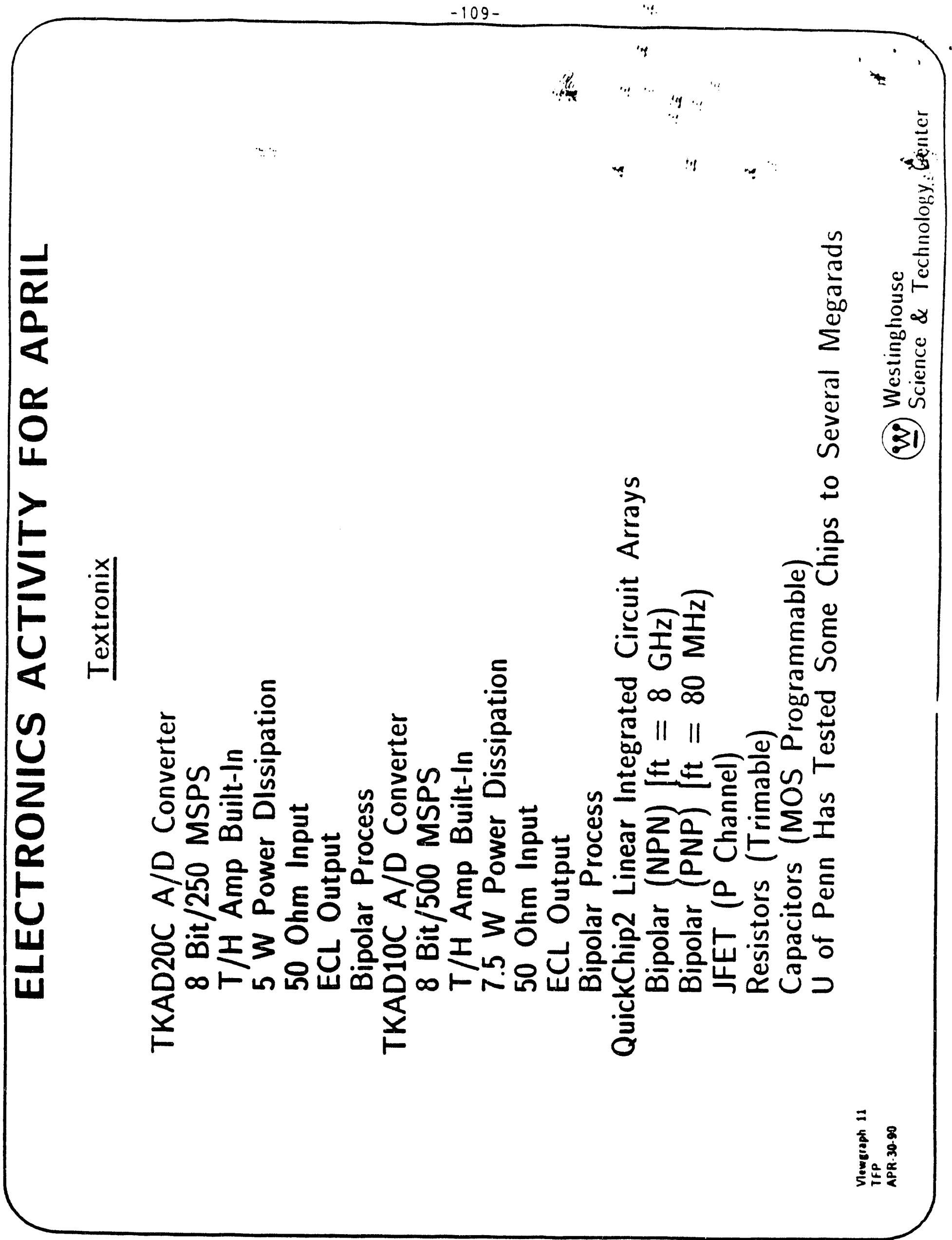




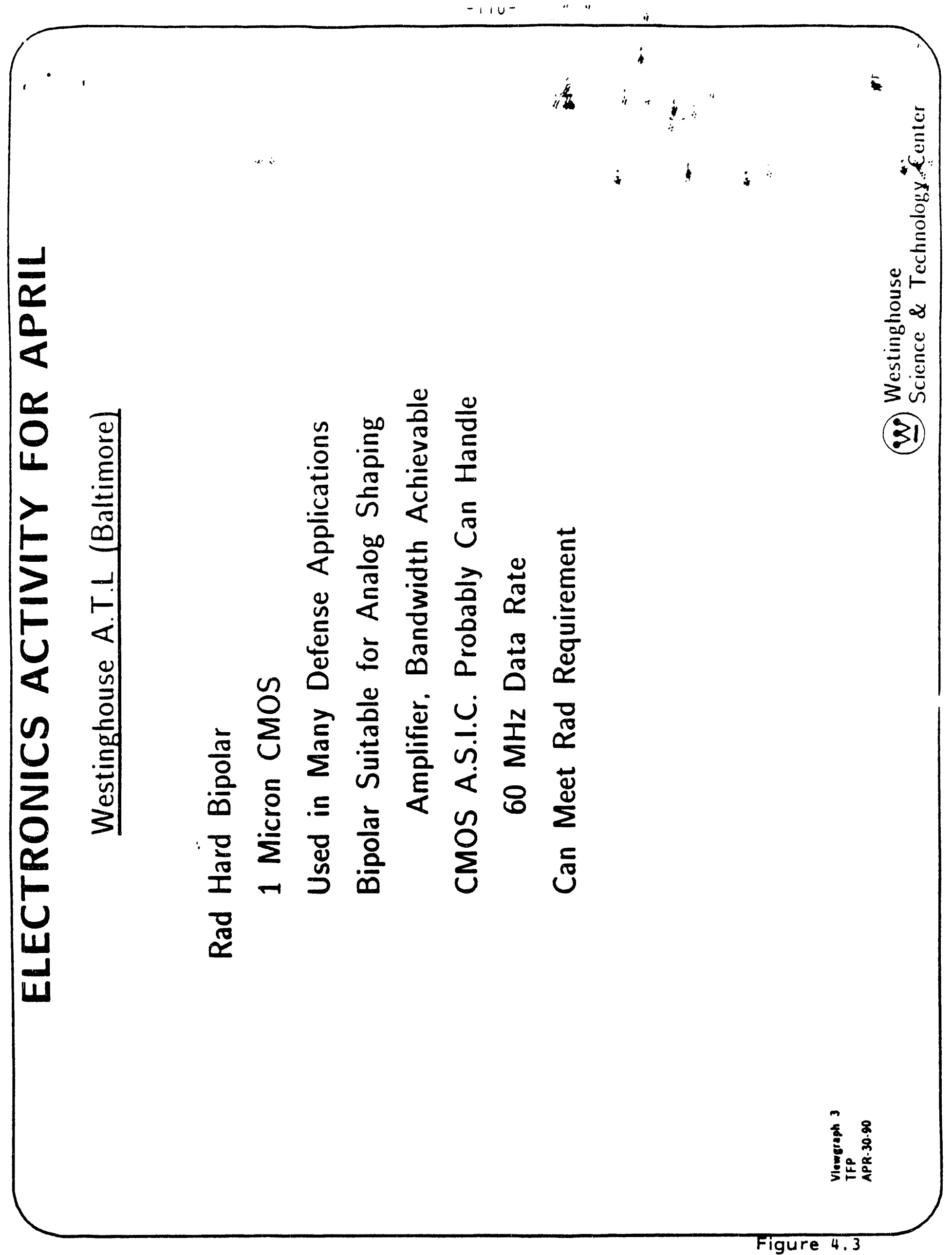




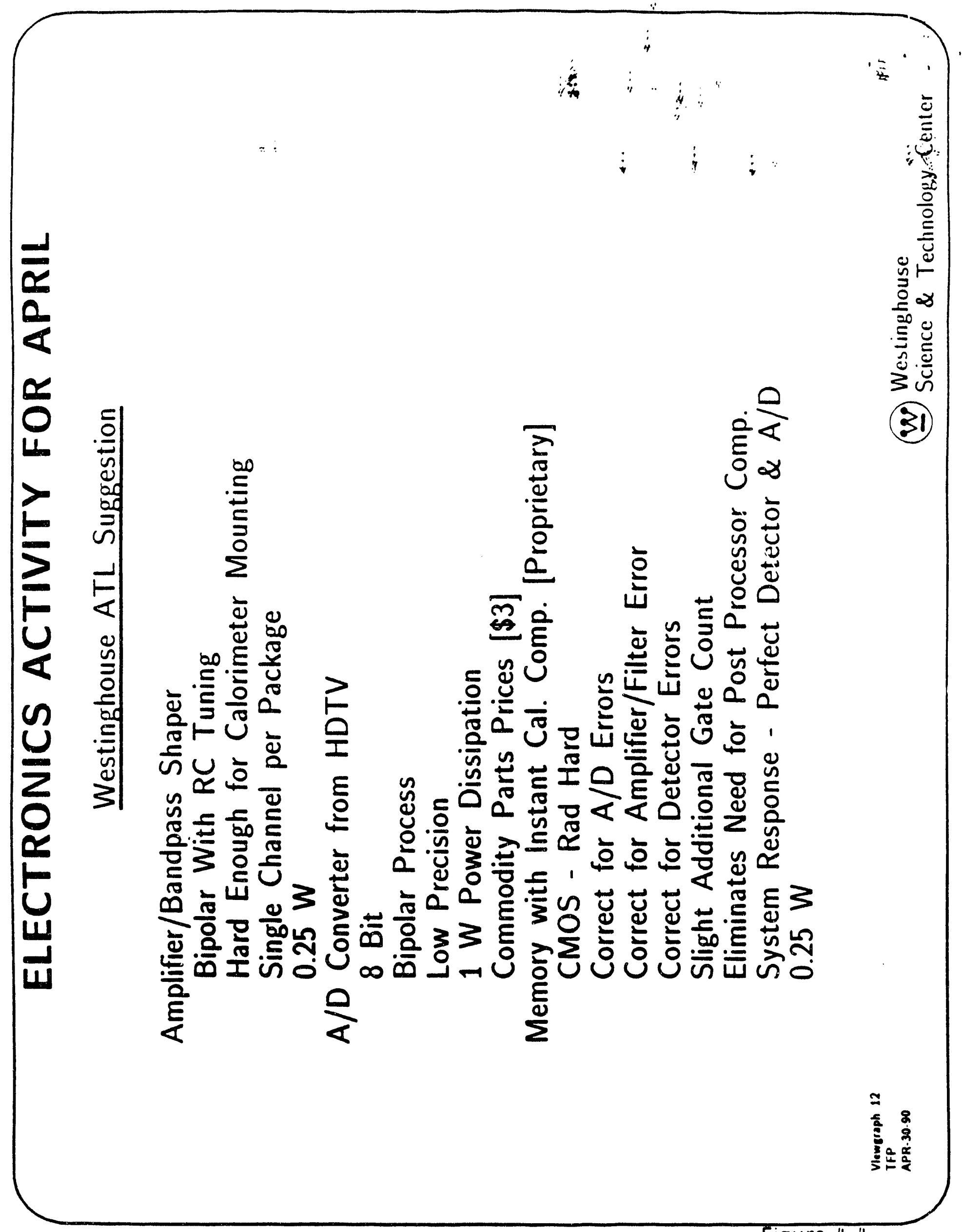




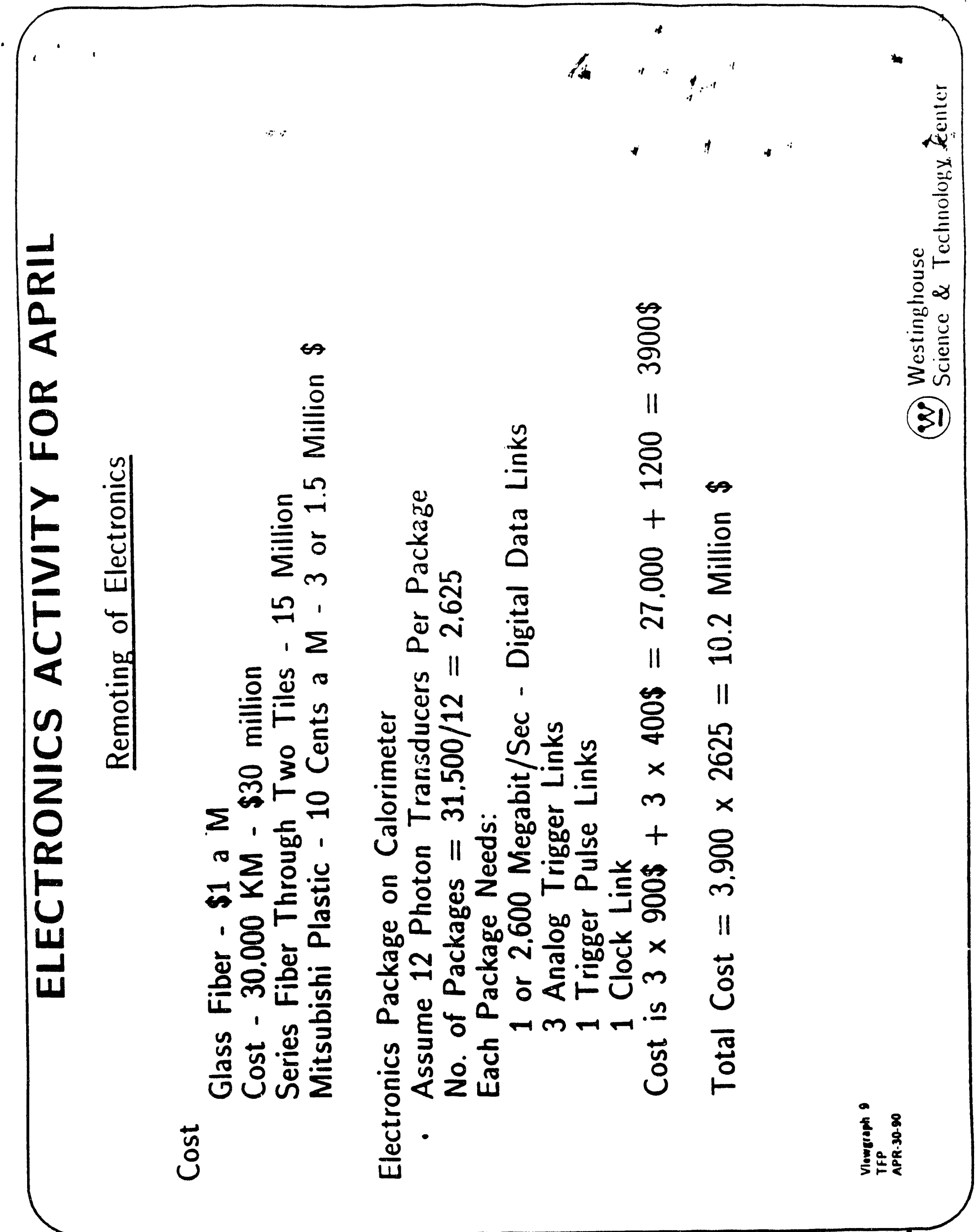


$-113-$
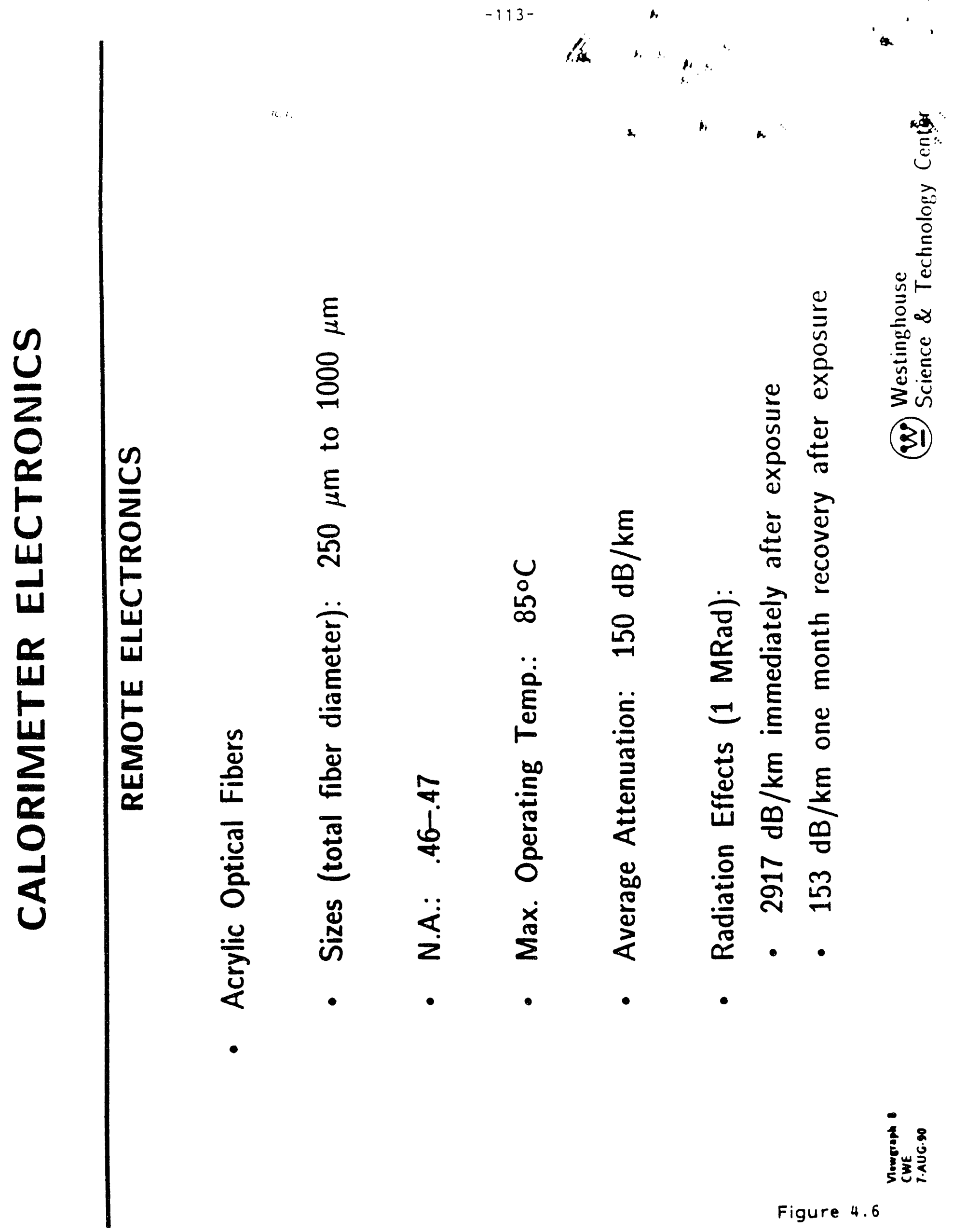

Figure 4.6 
$-114-$

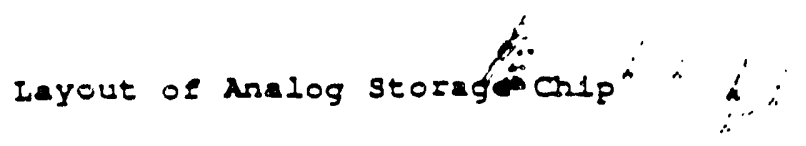
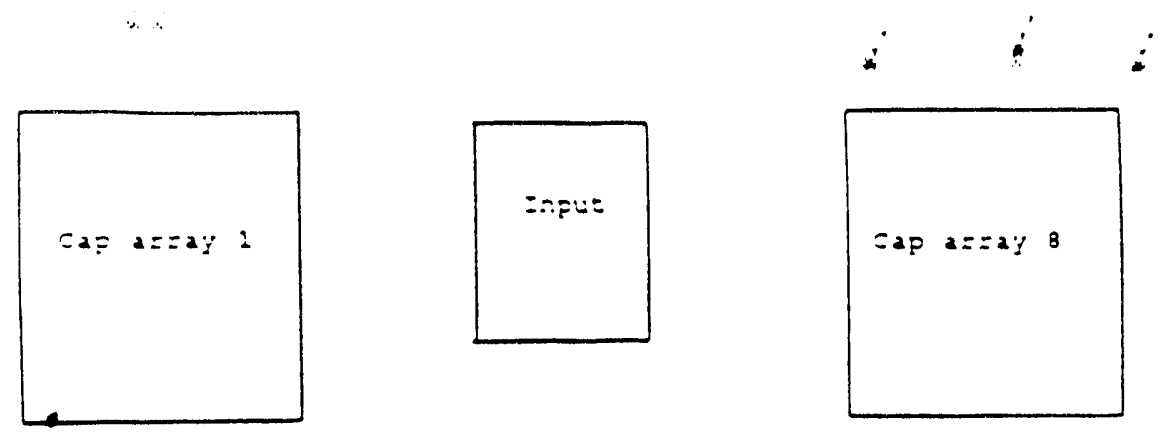

$\therefore$
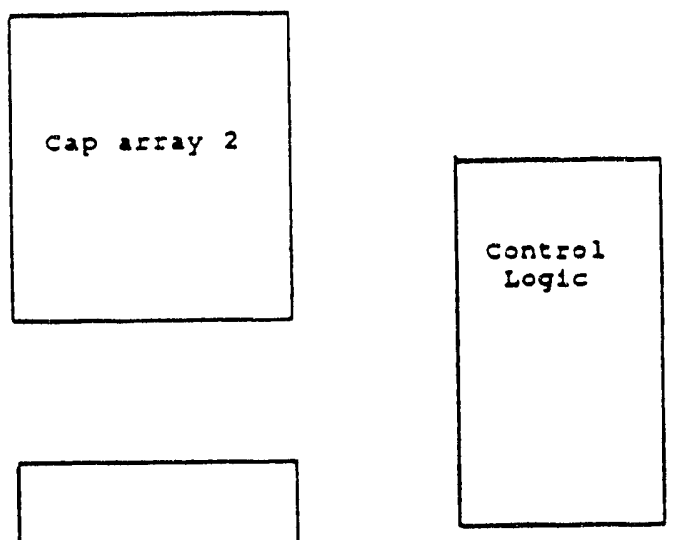

$=2 p \quad a= \pm 2 \% 8$
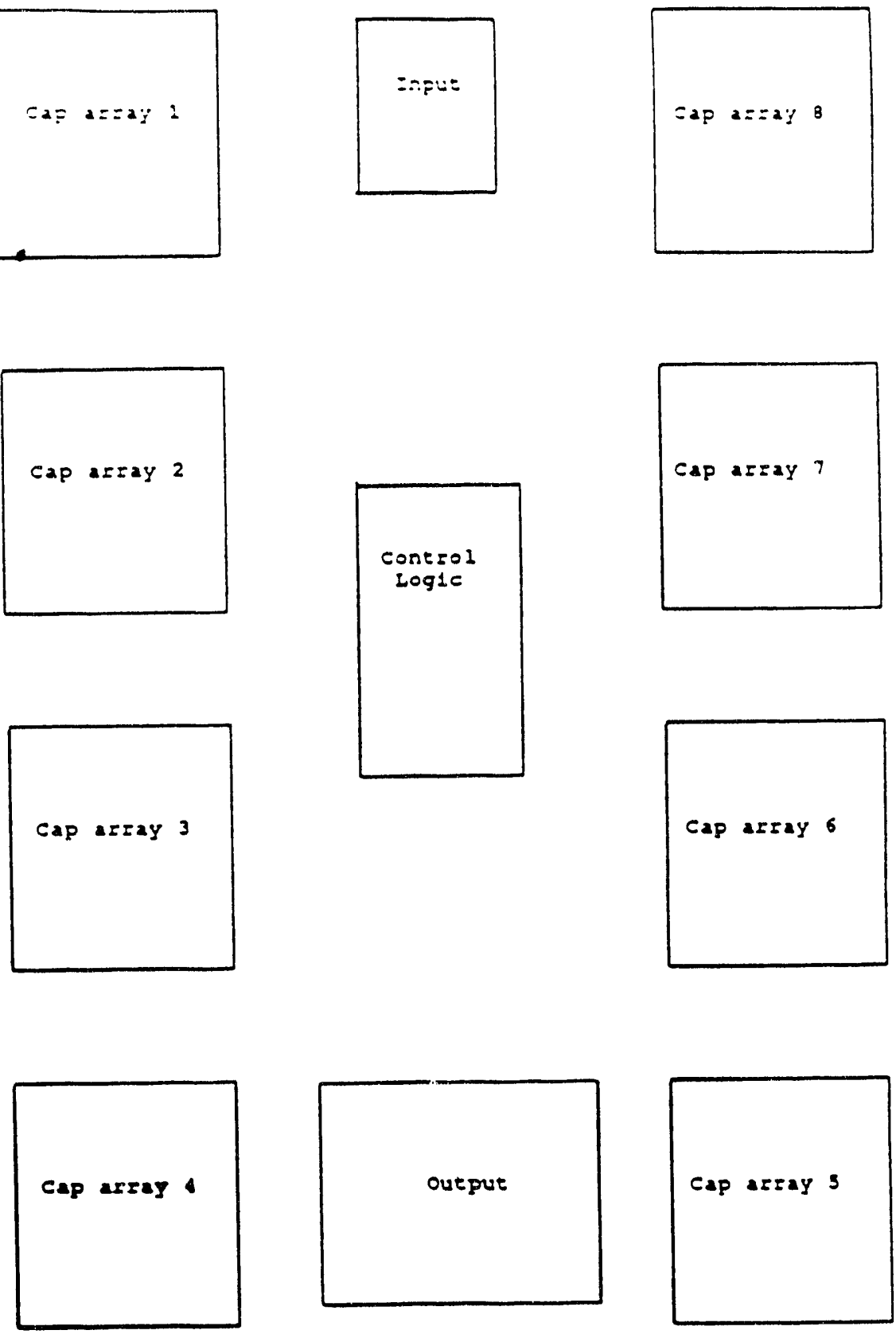

Figure 4.7 
$-115-$

Input curcust

x $\%$

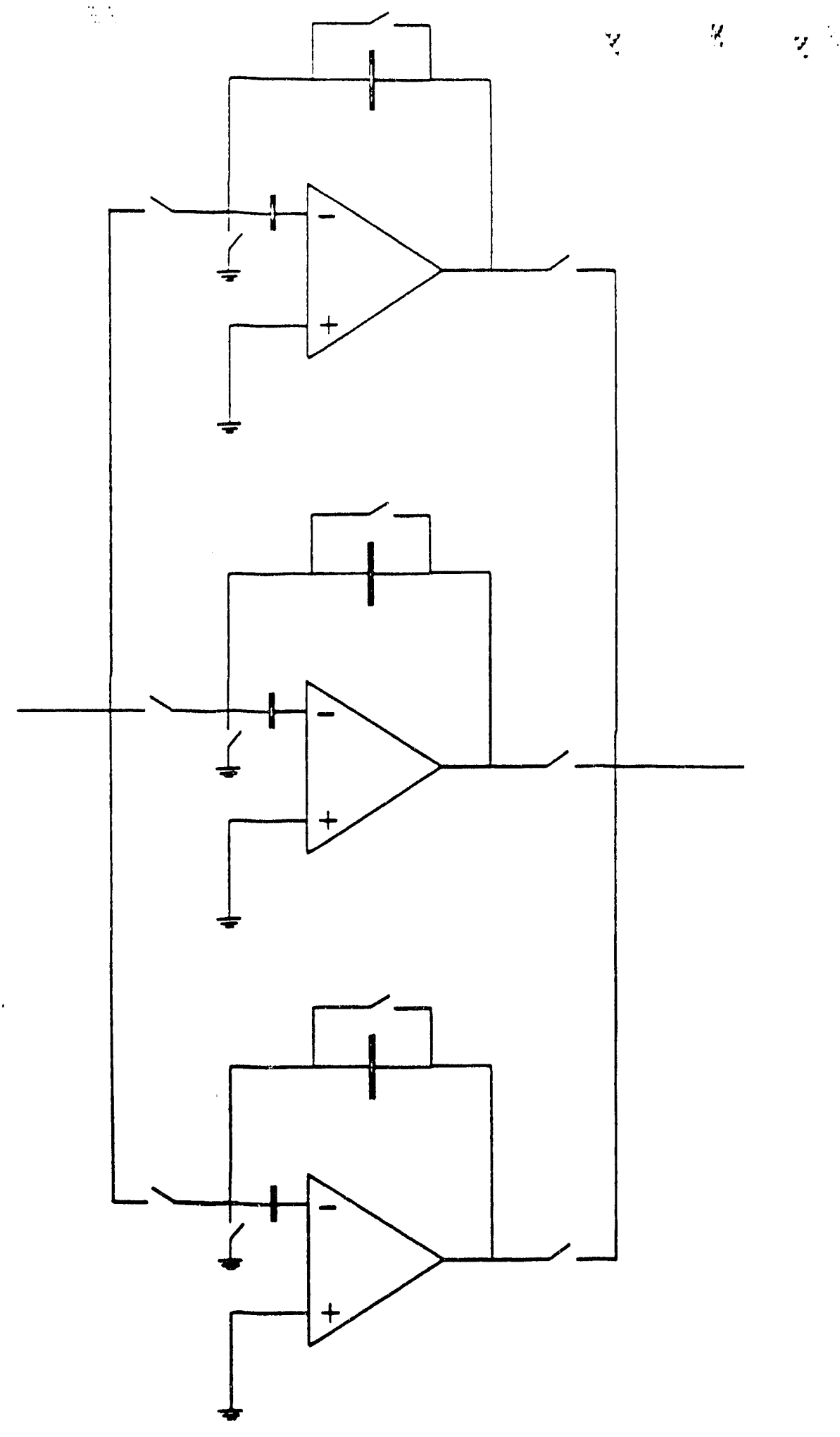

望

$\because$

Figure 4.8 
$-116-$

Switch setting while integratng $\quad \vdots \quad j$

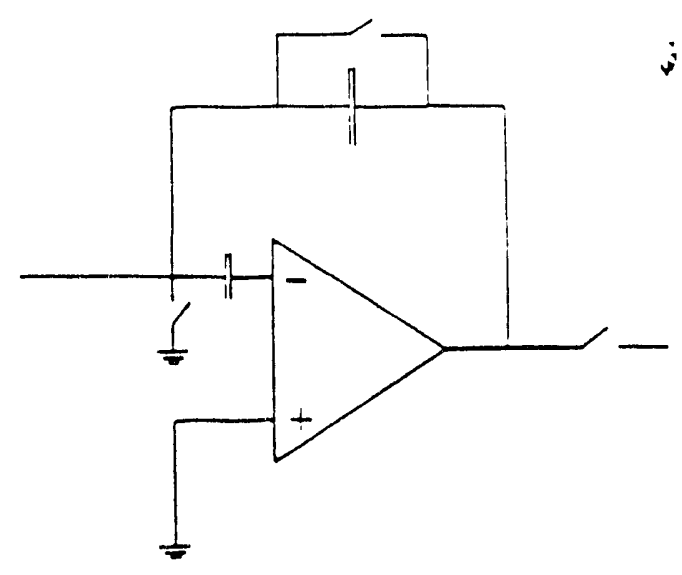

Switch setting while charging storage capacitor

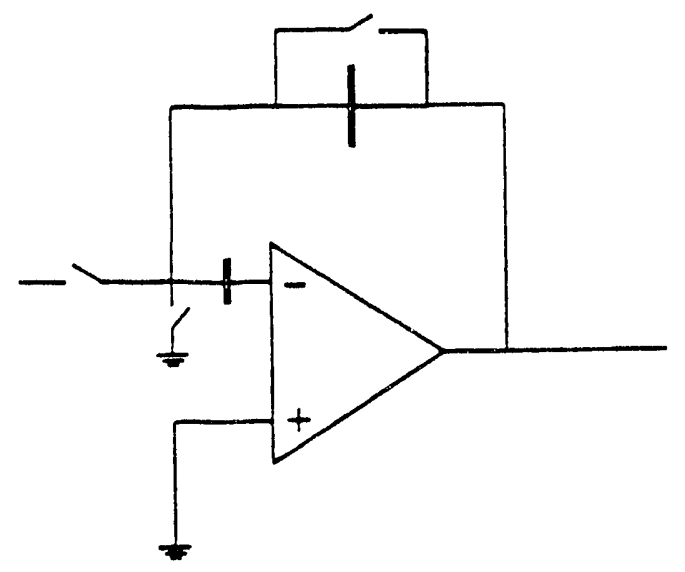

Switch setting while resetting integrating capacitor and recharging self referencing capacitor

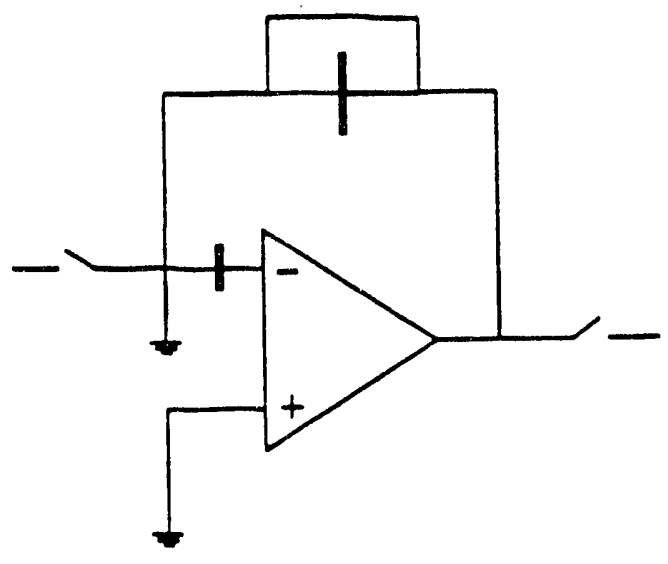

Figure 4.9 


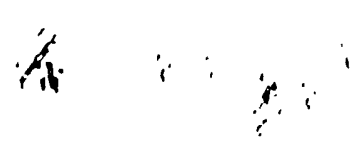

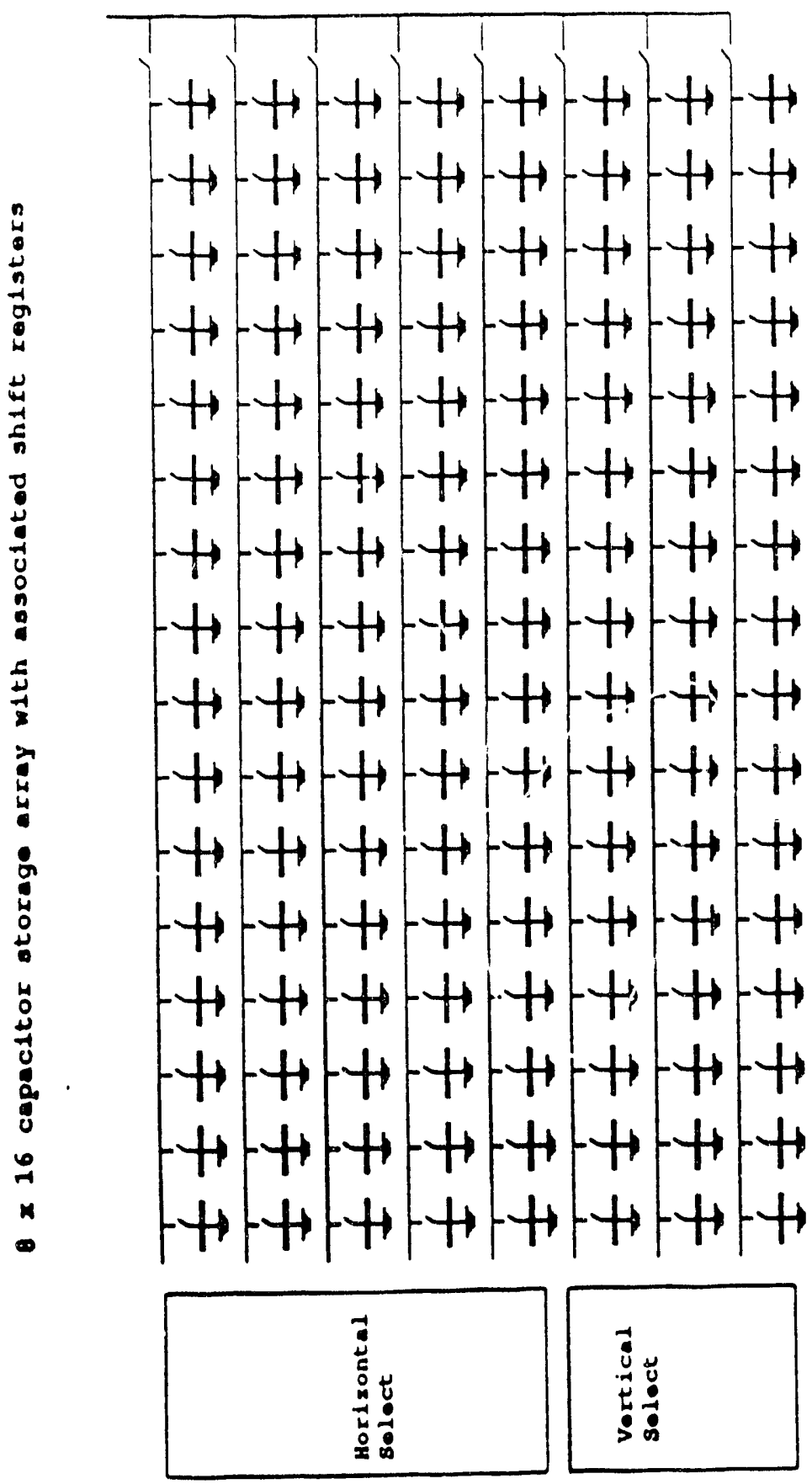


$-118-$

$\cdots$

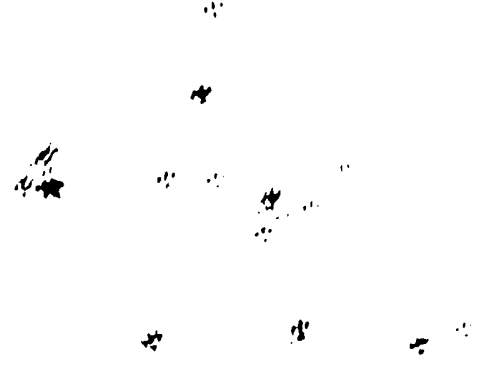

ousput eureuit
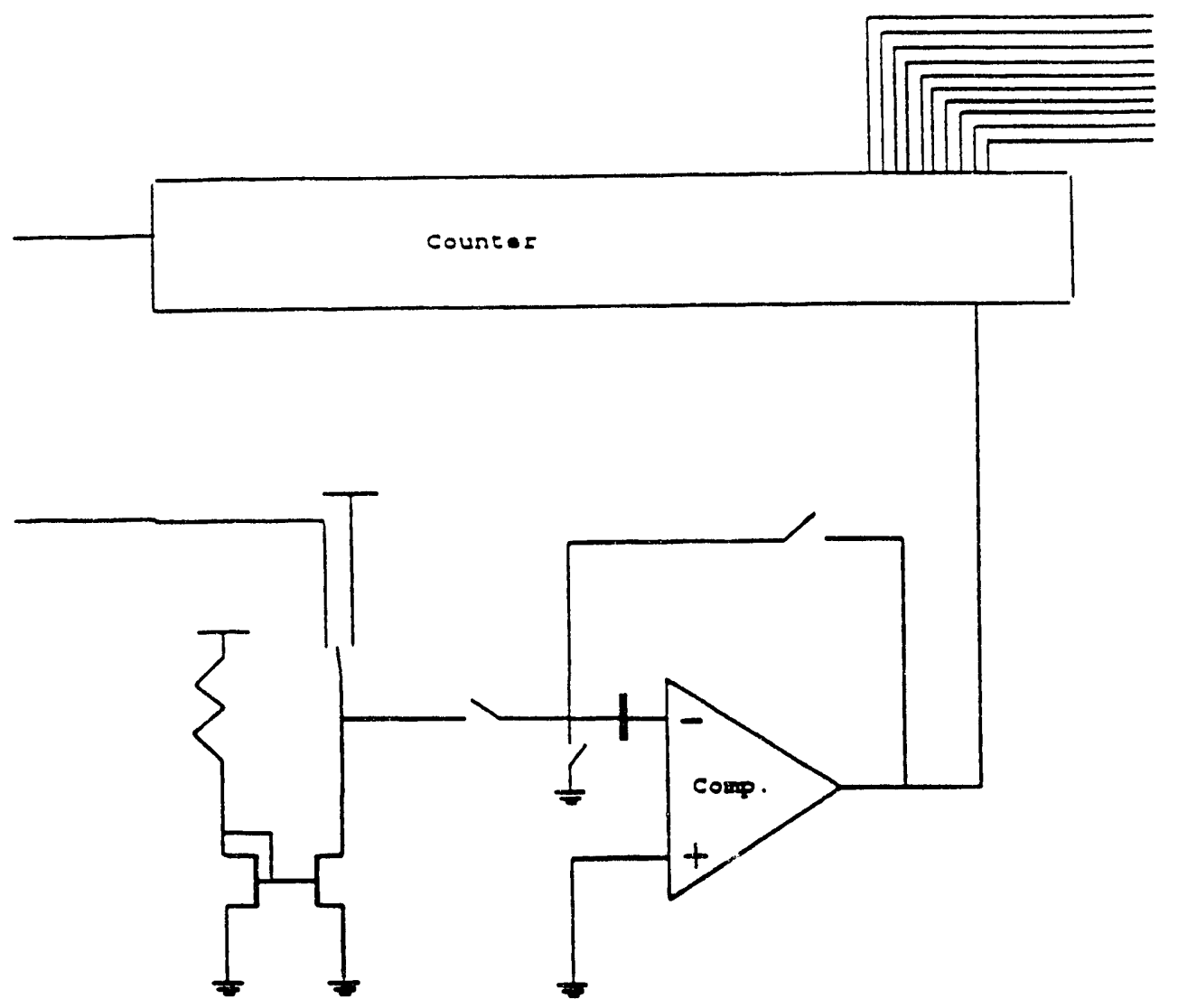

Figure 4.11 


\title{
BICRON CORPORATION
}

$-119-$
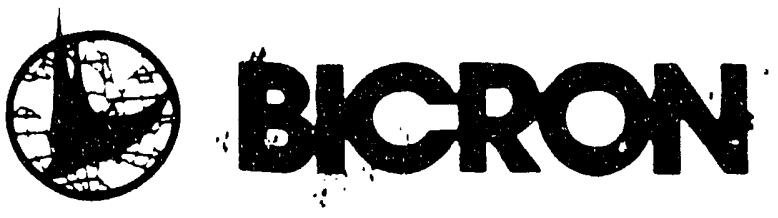

12345 Kinsman Road. Newbury. Ohio 44065 216.564 .2251 \& $\quad: \because \quad$ Telex 98047 i:

\author{
PROGRESS REPORT \\ SCINTILLATOR DEVELOPMENT PROGRAM \\ SCINTILLATING PLATE CALORIMETER SUBSYSTEY
}

27 August 1990

This reports on the Bicron work initiated at a planning weeting in $M a$.

RADIATION-RESISTANT SCINTILLATORS

In its SBIR fiber development program, Bicron had previously investigated novel plastic materials which might exhibit resistance tc gellowing from high dose exposure. The first of a series of materials of this type. designated RA-1. was fabricated into scintillating fiber and tested by Stan Majewskt on the electron beam facility at Florida State in December, 1989 . He reported its superior resistance to gellowing at the Tallahasaee workshop this spring. RH-l and its precursor, Type B, are actualig part of a larger program in which the material, RH-2, is the goal. The RH-1, being easier to make initially, served as a proof-of-principle step in the proje:t.

SHEET PRODUCTION

Most of the physical effort of the past $31 / 2$ months has been in casting RH-l scintiliator plates for supplying $10 \mathrm{~cm} \times 10 \mathrm{~cm} x$ $2.5 \mathrm{~mm}$ tiles to various collaboration members in accord with the schedule from the April meeting at Bicron. Difficulties were encountered 1 a making the transition from fiber preforms to sheets. The excelleat mechanical properties of the fibers were not reproduced in the sheets. The later were plagued by highly crazed surfaces and general brittleness. The actual polymertzation cjcle was time consuming. up to triple those for poljpinjltoluene (PVT) and polystgrene(PS) based scintillators. The several grall cale procesa problems with RH-1 material would be eliminated in a nass production system.

The second cartag, in July, fncorporated changes to deal with the crazing. Clear improvements were achieved, but the problem persisted.

Had gields been good, enough RH-l sheet was cast to supply over 400 tiles. 
In the aeantime, parallel fiber work revealed a possible chemicai weakness oi the RH-l when it was potted into a lead-fiber calorimeter module. This is being investigated separately. This new mpterp and the immediate need for large numbers of mechanicalig and optically reliable tiles has forced us to change directions.

RH-l sheet production will be abandoned, at least temporarily, in Eavor of a Bicron's RH-1 precursor. Type B, which was originaliy made in PS and was nearly as good as RH-l in the December fiber tests. The difference should be even less in the small tiles. Sheets will be immediately made with this formula in both PS and PVT. The first lots of 20-30 tiles in several casting variations, will be available by September $30,1990$.

Recent separate tests at Tallahassee showed PVT possibly more rad-hard than PS. The problem with PVT for plate tower calorlmetry is its mechanical weakness. Concurrent with the Type $B$ casting. Bicron will investigate methods of strengthening PVT sheet. A specific plan for this has been written.

\section{FLUOR RESEARCH}

1. A five-morith fluor development contract has been made with Dr. Joel Kauffman at the Philadelphia Coilege of Pharmacy and Science (PCPS). The prime $80 a 1$ of this work is the development of a spectrum shifting fluor for use in the WLS plates or fibers. This is to replace the fluor Y-7, which is also the same as the commerclal green fluor $R-27$. Y -7 has recently been characterized by Ir. V. Hagopian as relatively rad-soft.

The properties of the new fluor should be:

- Rad-hard

- Absorption spectrum: $430 \mathrm{~nm}-460 \mathrm{~nm}$

- Emiseion specirum: $500 \mathrm{~nm}-550 \mathrm{~nm}$

- High quantur efficiency, above $75 \%$

- Fast t1alag

- Smali self-absorpeion.

2. Bicron will undertake the development of a more efficient and wore rad-hard blue sintillator fluor system as an extension of its successful SBIR work on new gree scintillators for flber based tracking. 
Recognizing the need for improved blue scintillator tiles (for efficient optical watch to green wis waterials). Bicron developed a blue variant of the above green fluors which exhibits higher efficiency than traditional ones and which also should be relatively rad-hard. More of this particular fluor will be made for use in further testing and tile anufacture.

A second blue variant, which should be more rad-hard, is planned.

Schedules for this work are included on the attached Bicron Rad-Hard Sciatillator Program dated 18 August 1990.

SHEET PRODUCTION DEVELOPMENT

The sheet line has been totaliy torn down and refurbishment and rebuilding begun. The main structural frame has been sandblasted and repainted. The roli1ng drums have been stripped down and crated for transport to to a local company capable of performing the critical resurfacing. This represents approximately four man-weeks of 1 abor and $\$ 5 R$ expenditure to date.

\section{BICRON CORPORATION}

Charles R. Hurlbut

Marager, Organic Scintillators 


\section{SSC SUBSYSTEM \\ RAD-HARD SCINTILLATOR PROGRAM}

The Eollowing Scintillator development program will begin on

Eept. $\rightarrow, 1990$ with Bicron tianpower allocation as so indicated.

Tie $30 a l$ is to develop and demonstrace stable rad-hard plastic

scint:Lator and wils materials for sheet and Eibers by Spring oz

igyl providiag time for iterative testing and reports submizedin

late iggl. This work will build upon the foundation of knowiedze

in this field developed in the Bicron SBIR program.

A. CAST TILES FOR IMMEDIATE TESTING AND TOWER PROTOTYPE

Al Variants on Type B (precursor to RH- wat'ls.)

Bases: PS, PVT, RH-4, RH-j

Quantities: $20-3010 \mathrm{~cm}$ tiles each.

Shipment by:

ept. 30, 1990

Recast one or two best physical types

50 tiles minimum

Shipment by:

Nov. 15, 1990

B. POLYMERS

RH-1: Eibers for testing purposes.

The manufacture of large amounts of this material was never intended. RH 1 served to prove a point: that our general approach to formulating rad-resistant polymers is likely correct. Furchermore, we have recently discovered a specific weakness in its chemical properties which additionally supports our original plan that the ultimate rad-hard polymers would be the $\mathrm{RH}-2$ or $\mathrm{RH}-3$ materials.

$\mathrm{RH}-2$ :

Based on chemical theory, this should be the most rad-hard scintillator base. Because of this, we will make a strong effort to solve a difficult polymerization problem.

Process development

- Cross-linking for sheet production feasibility trials completed

Sept. 30,1990 Molecular weights

- High pressure polymerization feasibility trials completed

Oct. 15, 1990

Begin sheet casting trials Est. complete by

Oct. 1, 1990

Feb. 28,1991

Begin radiation trials

Time committment: $33 \%$ of full time basis

Jan. 5, 1991 Hayne Moser 
$\mathrm{RH}-3$ :

- Priority over RH-2

- Monomer synthesis, 2. 2 liter initially, 2 weeks fuli-time, starting with new lo, Complete date: (5-6 whs. calendar time)

Hayne Moser and Bill Harder

- Triai azpules

Pure and Type B fluor formula

Molecular weights

First small ampule samples out on

iov. i1, 1990

Begin R\&D preform casting trials

Nov. 20,1990

Begin sheet casting trials

Est. complete by

Dec. 1, 1990

Begin radiation testing

Feb. 28,1991

Jan. 5, 1991

C. FLUORS

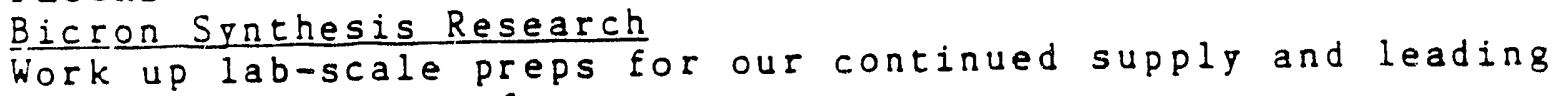
to large scale manufacture.

Bili Harder: 3 man-months $=6$ calendar months

- Nelta and Epsilon. First Priority

- Beta and Gamma

- V-2 and V-3

Perhaps $V-4$ and $V-5$, coordinate with PCPS work

- $P-2$ and $P-3$

Characterization

Photophysics:

Dilute and concentrated in toluene

Concentrated in PS

Light output, solutions and plastic scints.

Decay times of plastics

Wayne Moser: complete by

Nov. 30, 1990

Fiber preforms of best, starting

Dec. 1,1990

Update selection at that time

Radiation studies starting

Jan. 15,1991

D. FluOR detelopment at PBila. COllege of PHarmact

Primary Task: Develop efficient, high Stokesloss, fast waveshifter fluors with following shifting spectra:

$\begin{array}{ll}\text { Absorption } & \frac{\text { Emisston }}{430-460 \mathrm{~nm}} \\ 430-460 \mathrm{~nm} & 580-620 \mathrm{~nm} \\ 450-500 \mathrm{~nm} & 580-620 \mathrm{~nm}\end{array}$

Secondary Task: Rad-hard monomer research:

Date complete:

Jan. 31, 1991 
E. INDUSTRIAL FLUORS STUDY

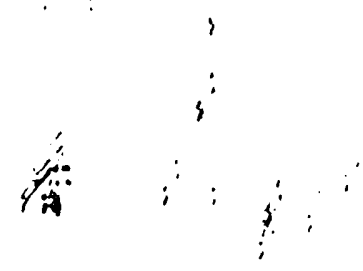

?hotopysics :

Approximately six fluors, green and red Absorbance: diluce and conc. in toluene

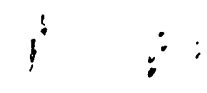
Emission:

As function of concentration in toluene Eront and backface spectra

In PS at 100 mg/1.

harne Moser: two man-weeks, complete

Nov. 30,1990

BICRON CORPORATION

C. Hurlbut

13 August 1990 
The first key lssue for a calorimeter based on plastic scintillator for an SSC experiment is that of radiation damage. For a calorimeter based on scincillator to be at all viable, it is essential to demonstrate the survivability of plastic scintillator in the expected radiation field at the SSC (of order 1 to 10 Mad over most of the central rapidity region). Initial cests of both blue scintillator plates and assorted fibers show that at the component level, significant progress has been made in this area this year. For example after 1 MRad of irradiation followed by 22 days of annealing, a scintillator plate based on the Bicron RHI plastic showed no measurable change in either attenuation length of initial light output (within a $1 \%$ measurement error). Similarly, a fiber based on the green scintillator 3 HF showed only a $10 \%$ decrease in attenuation length and an $8 \%$ loss in light output after an irradiation of 3 Mrad followed by a few days of annealing. These successes coupled with the fact that scintillator manufacturers are betcering their understanding of the damage and recovery process and thereby hope to significantly improve on this performance demands that we continue co aggressively pursue this approach to calorimetry at the SSC. We are therefore so proposing in our Fy9l plan and in addition incend to acquire a deeper understanding of the system dependent issues of radiation damage on the performance of the calorimeter.

The choice of absorber is the second key issue for our design of calorimeter as our staced goal is compensacing calorimeter with high precision and uniformity. Our imulation studies have shown that both depleced uranium and lead absorbers of appropriace thicknesses relative to the chosen thickness of the scincillator place can yield a calorimeter which has equal response to 
both hadrons and electrons or photons of the same energy. A calorimeter based on a pure iron absorber does not. In addicion, the ime dependenéof compen-

sation in both lead and deplezed urarium systems is very similat in the

initia: fart : : the shower and both can yield a response which is almost com-

perisating in less than the 16 nsec between bunches at the SSC $(e / h<1.05)$.

Therefore, even crigger signals for chese calorimeters measure an essentially

compensating response. Energy release from delayed de-excication of neutron

capture reactions is observed only in the uranium system and may be the

physics reason to prefer lead. This gives approximately $3-10 \%$ of the total

signal strecting out over 200-500 nsec. Although we have not yet quantified the experimental effect of this energy, such pileup is very undesirable (and yet more so at the higher potential luminosities). Mechanical solutions for both choices of absorber have been evaluaced using both analytical and simulation techniques. Both absorbers are feasible with the caveats that both allow azimuthal load transfer in the body of the calorimeter and that in the case of lead it is embedded in a composice structure. This structure is estimated to comprise at worst < $10 \%$ of the lead volume and potentially can be distributed where it will have least affect on the performance of the calorimeter (this is part of the work being proposed for FY91). Moreover, the fabrication process of lead casting and the cost of the raw material greacly favours lead over depleted uranium. It is therefore our assessment that the appropriate choice for the absorber is lead and lead/composite and that the fabrication process to be used should be casting. Our proposal of work for fygl assumes this conclusion and is directed at demonstrating this conclusion with physical devices. 
Two options for the optical system used to couple scintiliation ligtt Erom the plate to the photon iransducer were conceptially évaluaed. wavelength shifter plate is a very well known and well understood approacn ard for it. Ore the serious limitation for waveshifter plate is ln ire necessity to allow azimuthal load transfer between modules and therefore ihe need io modify che plate cross section at these radii (with resulting serious implications for both laceral and depth uniformity). A second serious Limitation is the dead fiducial volume associated with the waveshifter plate and its associated air gaps and tolerances to preclude its being crushed. This loss can amount to as much as $7 \%$ of the surface area and alchough energy measurement in this region can be obtained by a suitable tilting of the from section, high quality electron measurements in this region would probably be impossible. The third serious limitation of this approach is Cerenkov light in the wavestifter place icself. Although this can again be largely removed by tilting the front section of the calorimeter, this design, by using single sided readout to reduce dead space, has no intrinsic capability of identifying such 1 ight and thereby of vetoing the associated spurious signals. On the other hand, readout via embedded wavelength shifting fibers can potentially remove all of these limitations and in addicion could be used in a design to allow refurbishment of those parts of the calorimeter exposed to the greatest radiation damge. However, it has very large potential for intrinsic nonuniformicy. The Fermilab group lead by $G$. W. Foster and J. Freman have completed some early cests using this optical approach and have had considerable success. It is our assessment that this approsch is the one with greatest potential for achieving our stated goal of maximum hermeticity and fiducial volume and we therefore propose it, as the favoured choice, as the one to be allocated our resources in FY9l. It is nonecheless true that should we demonstrate that scincillator itself is radiacion hard, then should we fail 


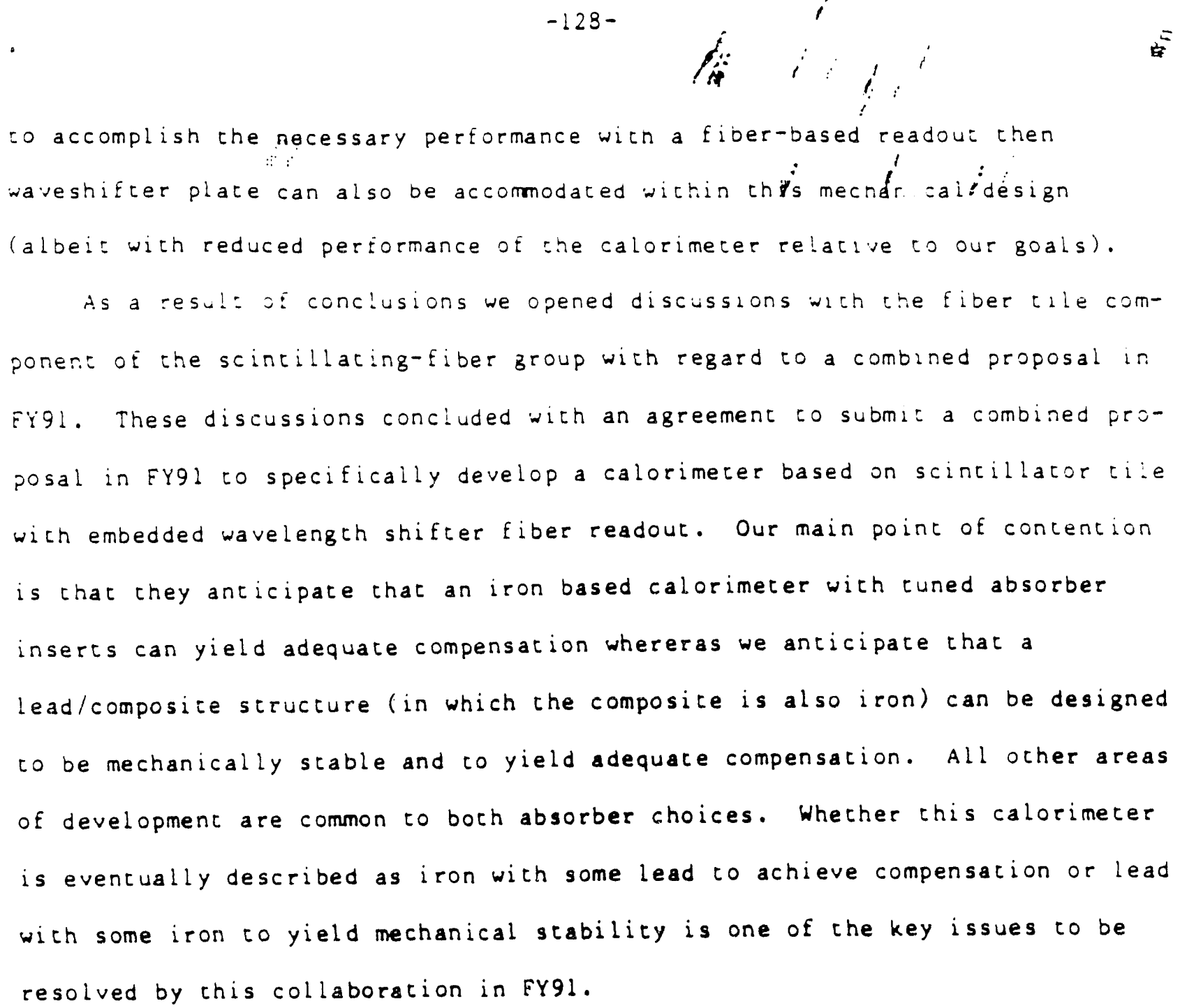




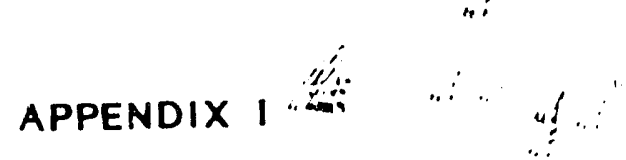

Report on Benchmarking CALOR at A.VL

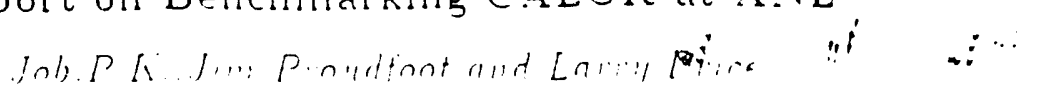

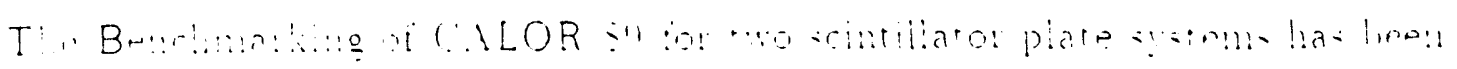

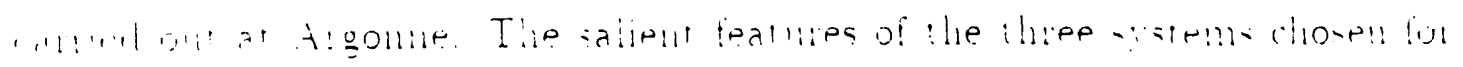

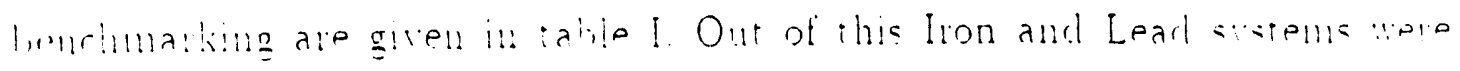
al1

All ralculation were done for 10 Ciel pions and elcctroms. The enely: ill unet for hadrons in HET(: is 1.0 Mel. The energ! cur used tor electrons in ECis is 1.j Mel and for photons 0.1 Mel. The ESTEPE parameter in E(is in not used. Teutrons below 20 Met were transported by MORSE. The low puegr photons from EGS were not mansponted by MORSE becanke of thrir urgligible effect on the energ! cleposition ( less than $0.2 \%$ ). The light

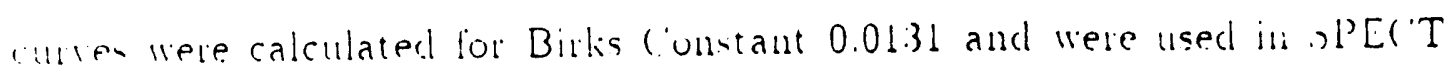
a III MORSE.

The electron response plot. longitudinal shower distribution and wo di. monvional shower distribution were calculated using E(i.s on a ('R.A) -.X.MP. The ('ALOR sulte of codes were lun on an IB.M 30St. The erentwise enterg." deposition in the scintillator plates from each of the three codes of ('ALOR (HET('. E(iS, and MORSE) and their arerages were calculated and storel in separaie files. From these files the response plot for pions were calculated by a uscr written program.

Table II gires the summary results. The response plots for pions and electrous. their resolutions and the e/h ratio for both the configurations are given. These ralues are to be rerified independently by the other groups. 
T.ABLE I

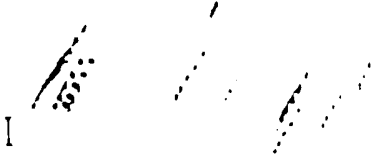

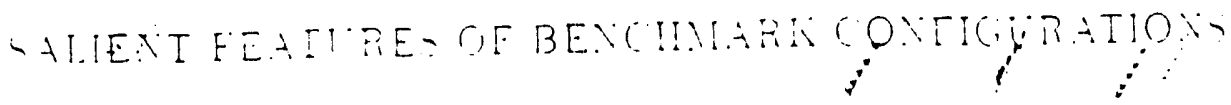

\begin{tabular}{|c|c|c|c|}
\hline & $1 ! ! \cdots 1 \because$ & $P 1, \ldots 1: 12$ & Fo. $11:$ \\
\hline 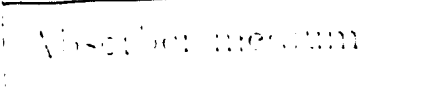 & 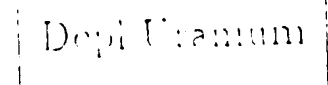 & Lead & l:o:! \\
\hline 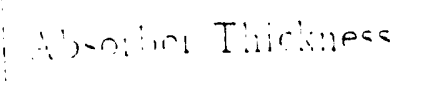 & 0.336 & D. & $1.76:::$ \\
\hline Ranition Len:?!! & a $3.3 \mathrm{con}$ & $0 . j 0 \mathrm{c}: \mathrm{n}$ & aris \\
\hline Virron lan kag:h & $10 . j \mathrm{cos}$ & $10.70 \mathrm{~cm}$ & $\therefore-09)(2)$. \\
\hline Den-ii: & $1 \leq 179 ! n \cdot \mathrm{cm}^{3}$ & $11.35 \% 121 \mathrm{~cm}^{3}$ & $7 \therefore \overline{g m} \mathrm{~cm}^{3}$ \\
\hline Ximmier Densil! & $\frac{1}{4}+11+10=2$ & $3.2(1))^{x}=10: 2$ & $\therefore 4 \div 601022$ \\
\hline tcli: e medium & Scimbillator & Scintillator & Sciutillator \\
\hline Scrimilator Thickness & $0.25 \mathrm{~cm}$ & $0.25 \mathrm{~cm}$ & $0.25 \mathrm{~cm}$ \\
\hline Drusity & $1.0+\mathrm{g} m \mathrm{~m}^{\prime} \mathrm{cm}^{3}$ & $1.0 \mathrm{lg} \mathrm{g} / \mathrm{g} / \mathrm{cm}^{3}$ & $1.0 \pm \mathrm{gm} ; \mathrm{cm}^{\circ}$ \\
\hline 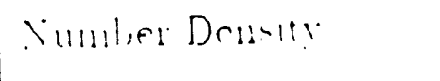 & & & \\
\hline ('arlion & $4 \therefore 110: 2$ & t.ミ1 $10^{22}$ & $4.51 * 10^{22}$ \\
\hline Hurliogen & $+1.2 \times 10=2$ & $1.9 .2 \times 10^{22}$ & $+.92 \cdots 10 \div 2$ \\
\hline Birkin ('onstall & 0.0131 & 0.0131 & $0.01: 31$ \\
\hline Cilubal Dimemsions & $100 \cdots 100 \mathrm{~cm}^{2}$ & $100 \cdot 100 \mathrm{~cm}^{2}$ & $100 \% 100 \mathrm{~cm}^{2}$ \\
\hline Depril & $\therefore \lambda_{1 \ldots 1}$ & $s \lambda_{1, n t}$ & $\sum \lambda_{1,1}$ \\
\hline
\end{tabular}




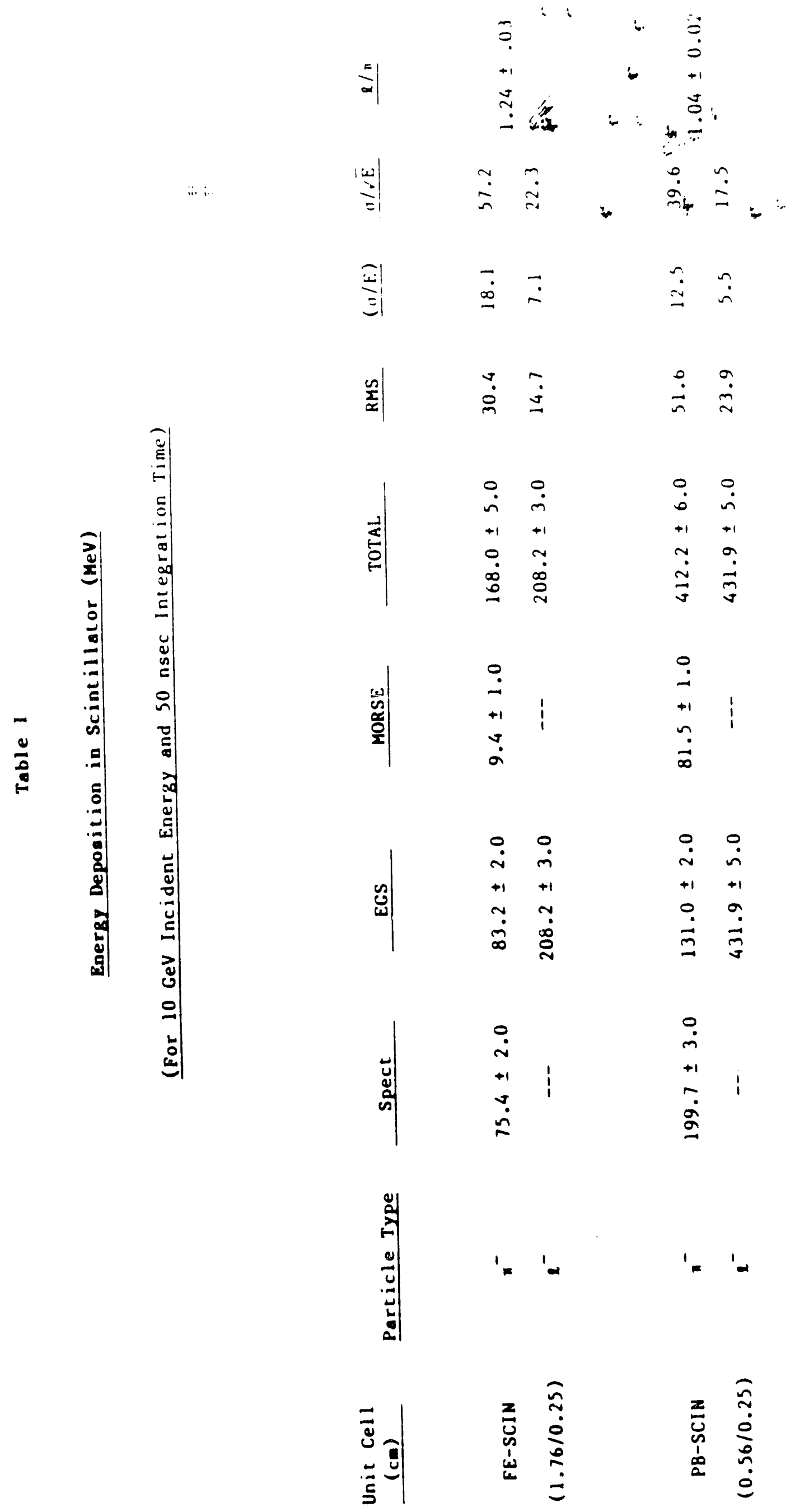




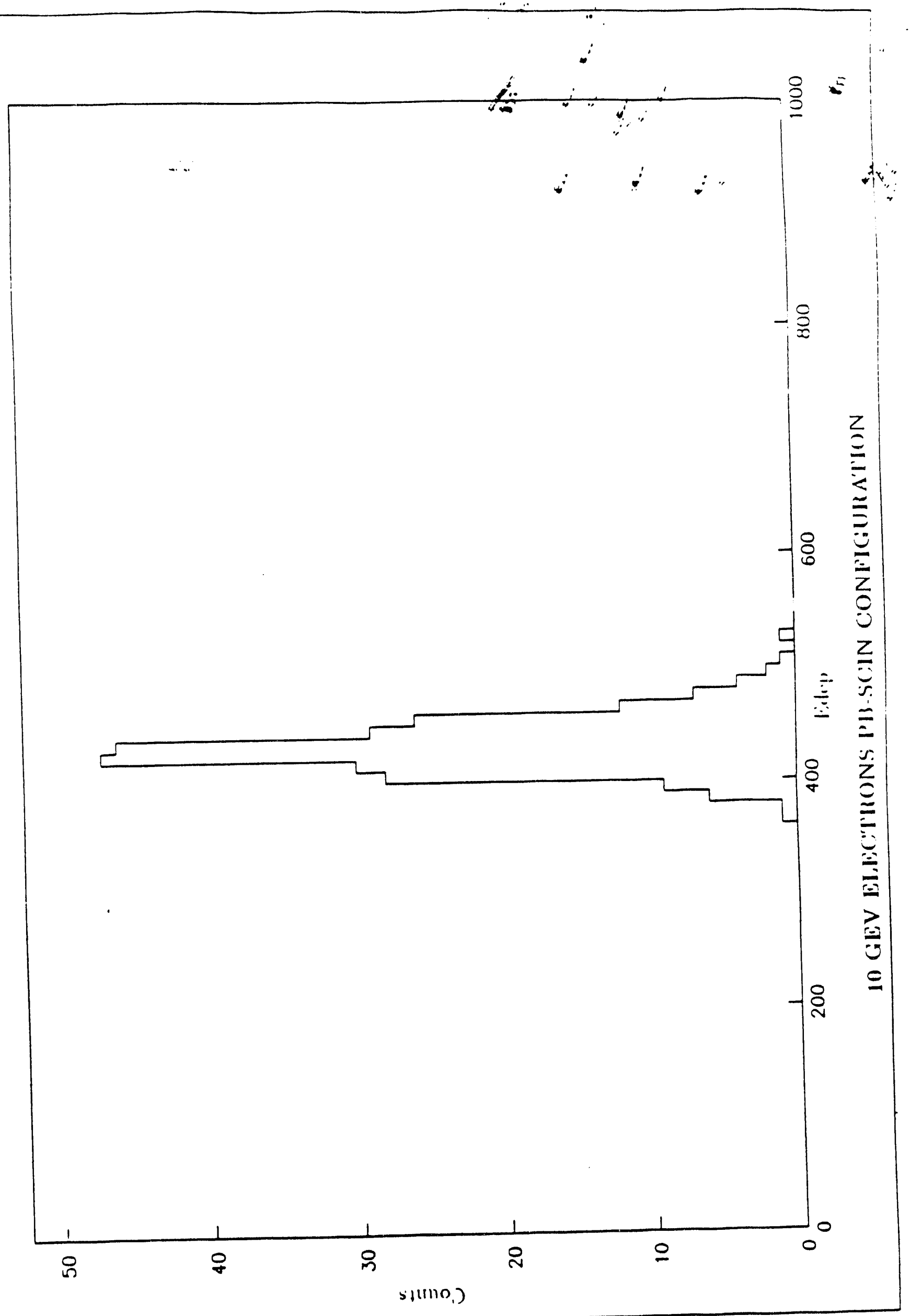




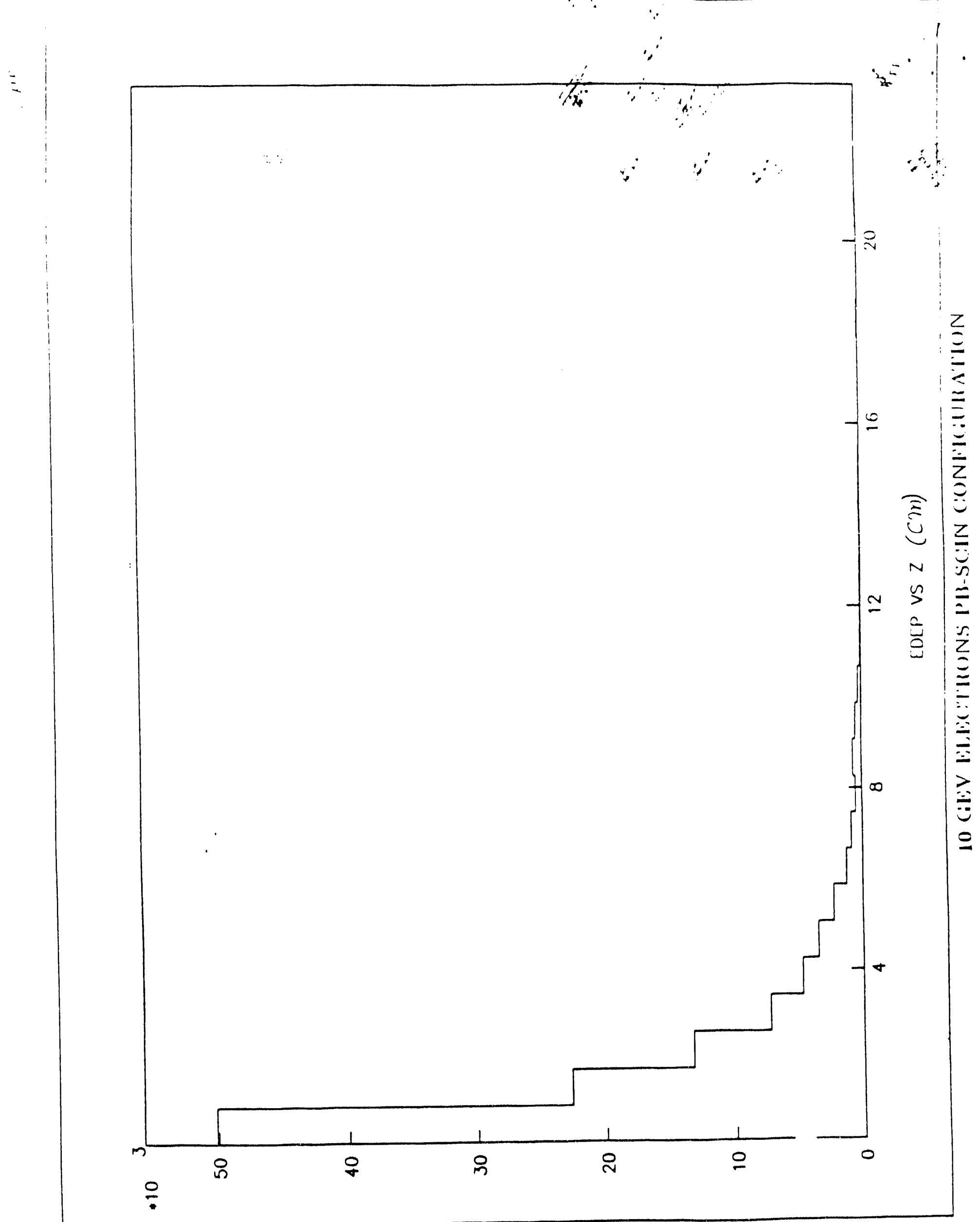




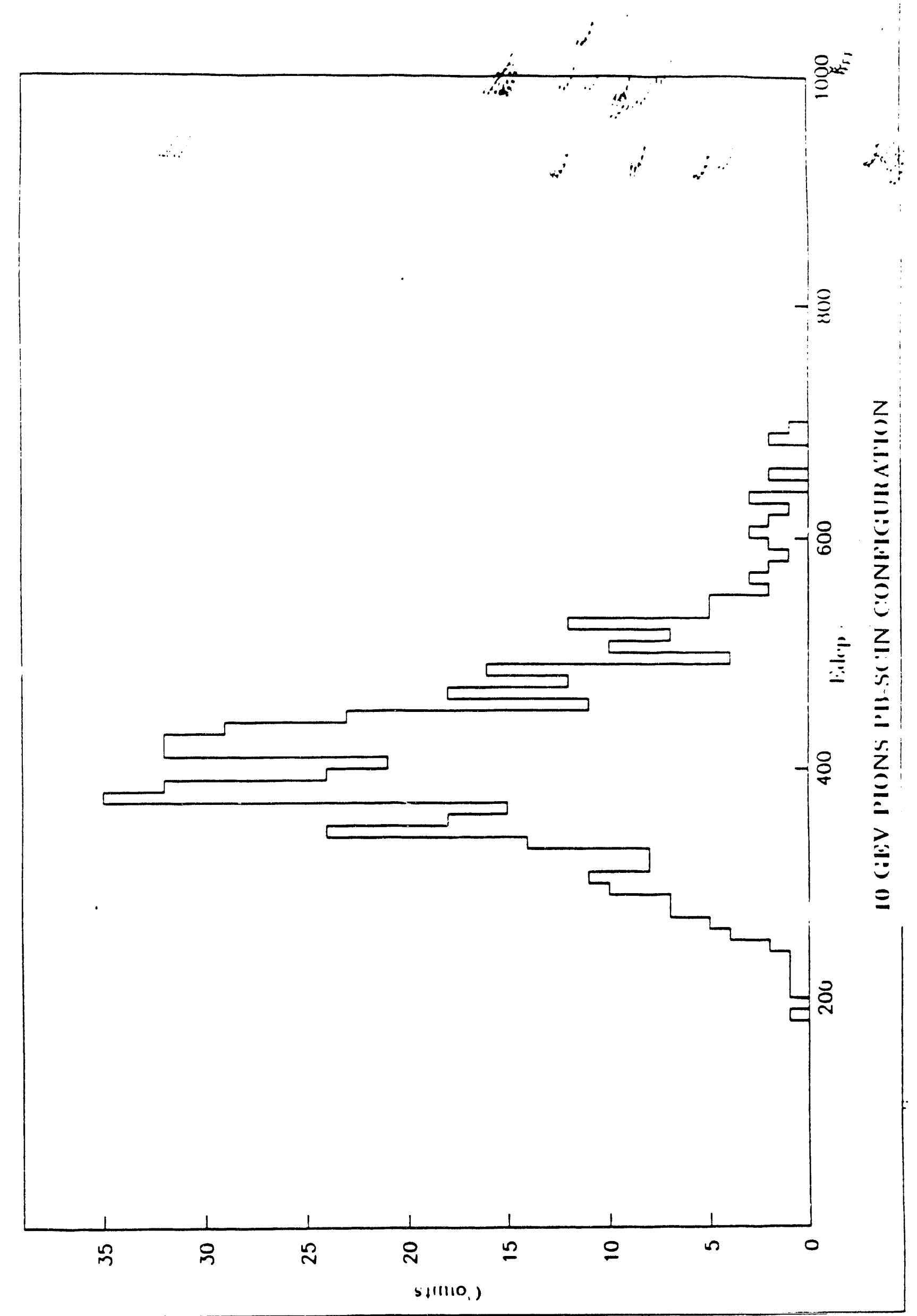




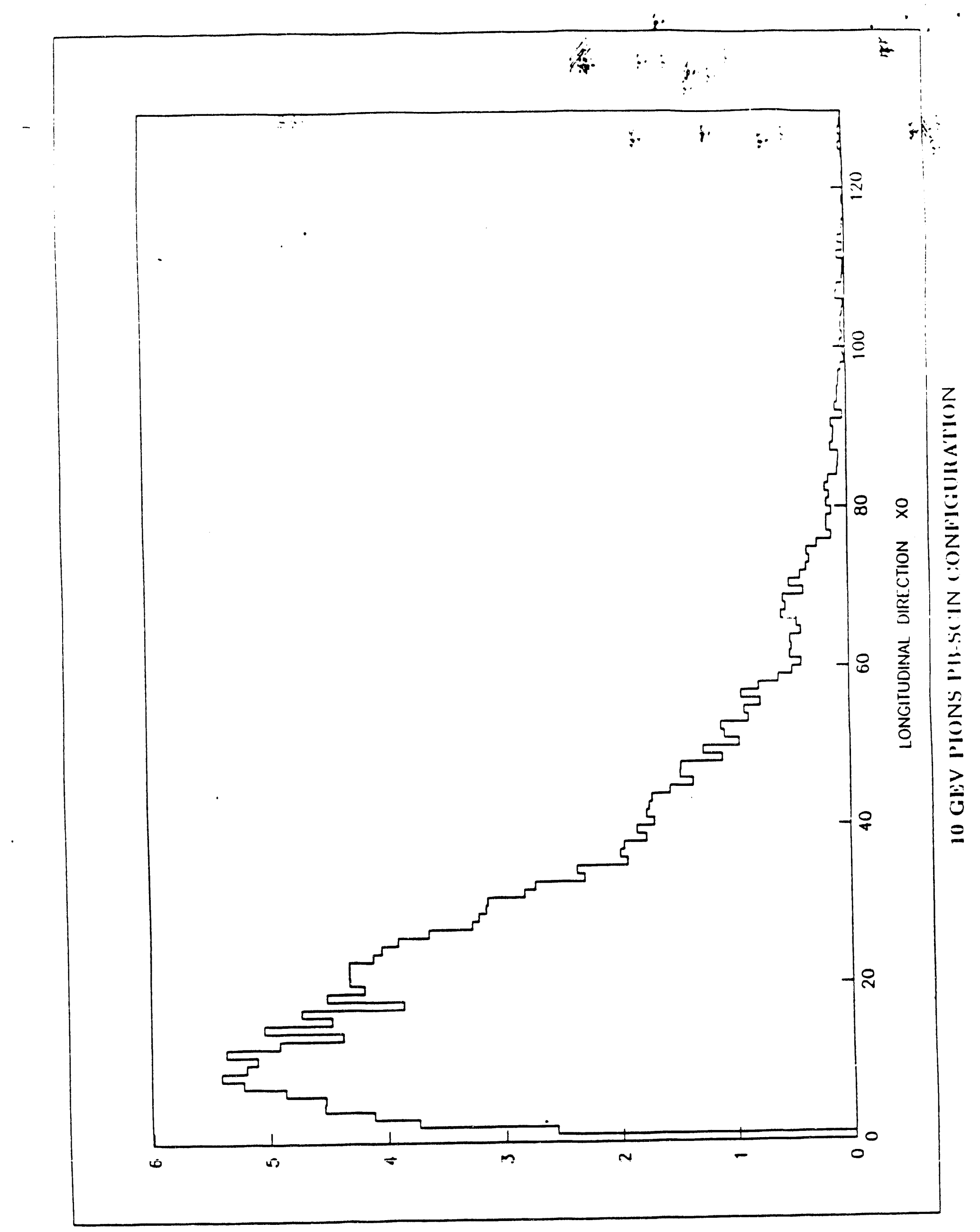




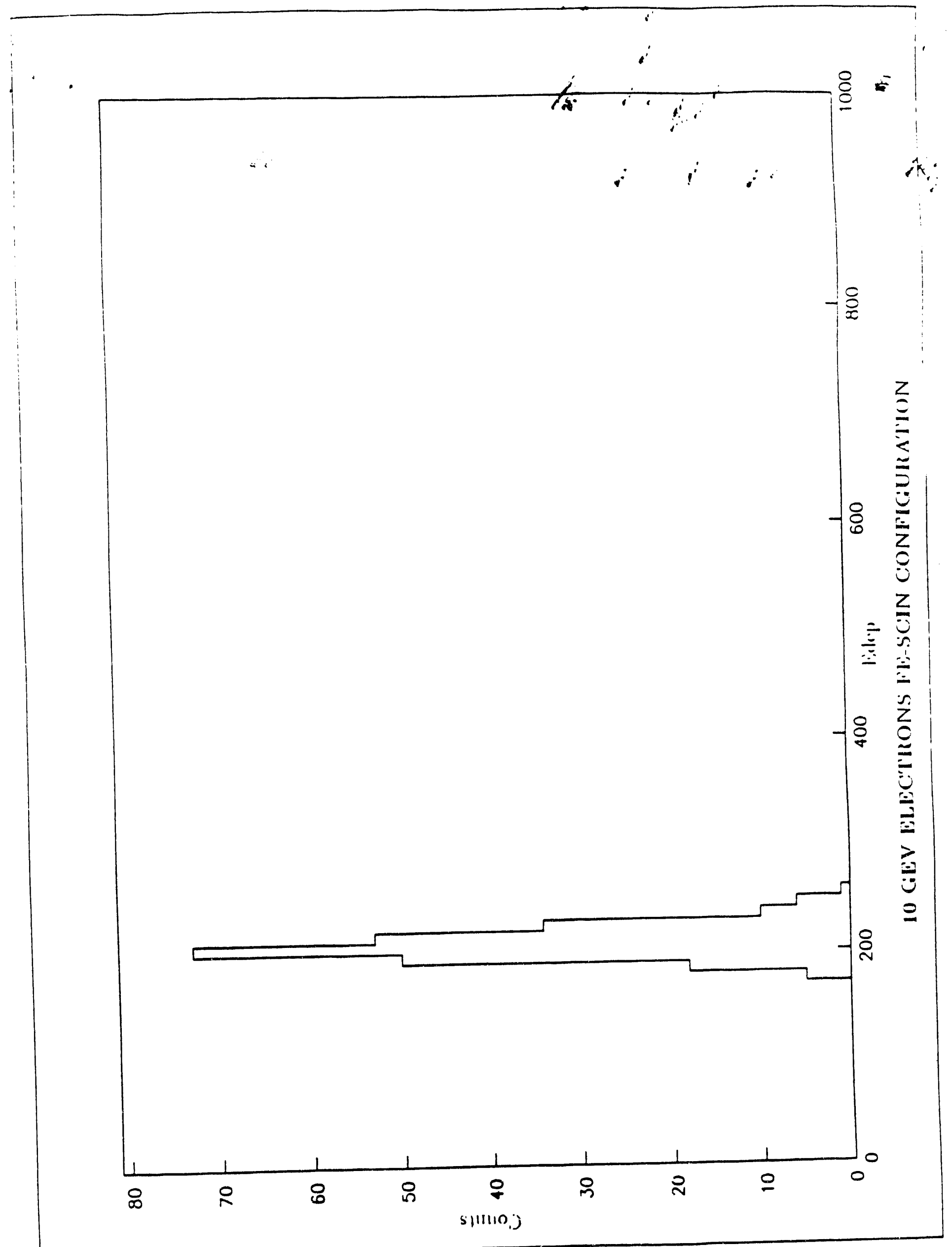




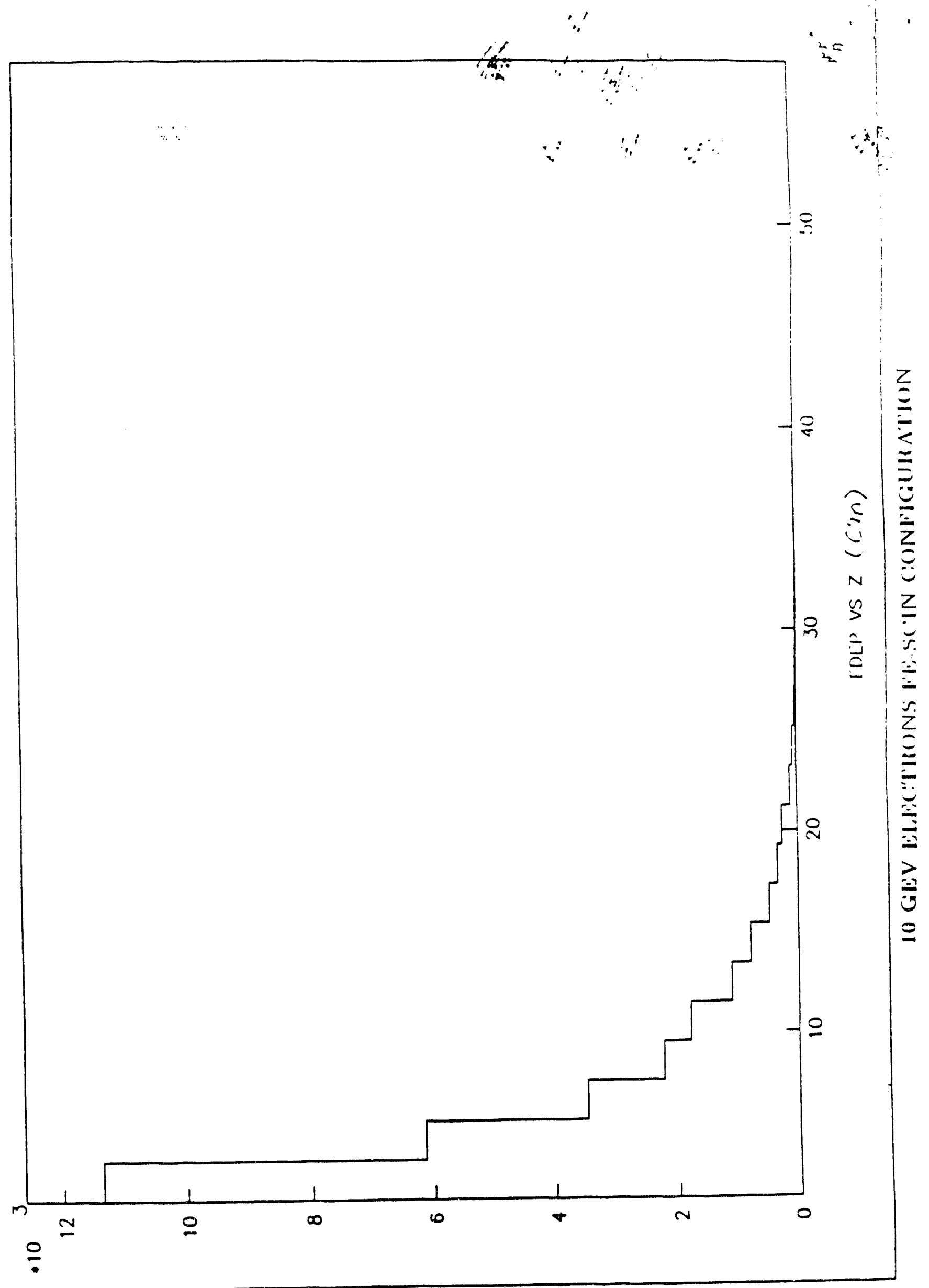



魚 $\because \because 9$
$\because \quad \because{ }^{\circ} \quad \because$
$\because \because$

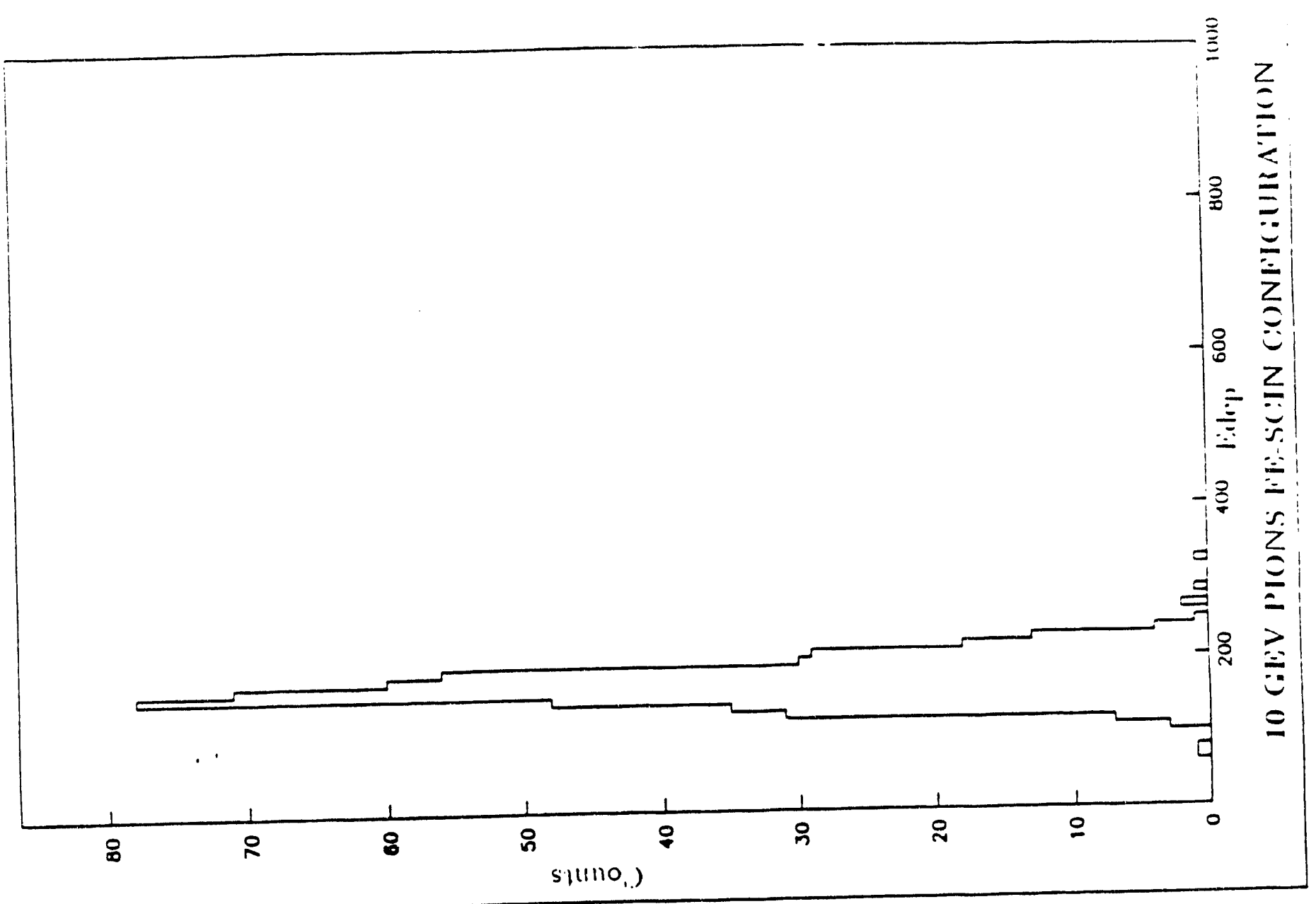




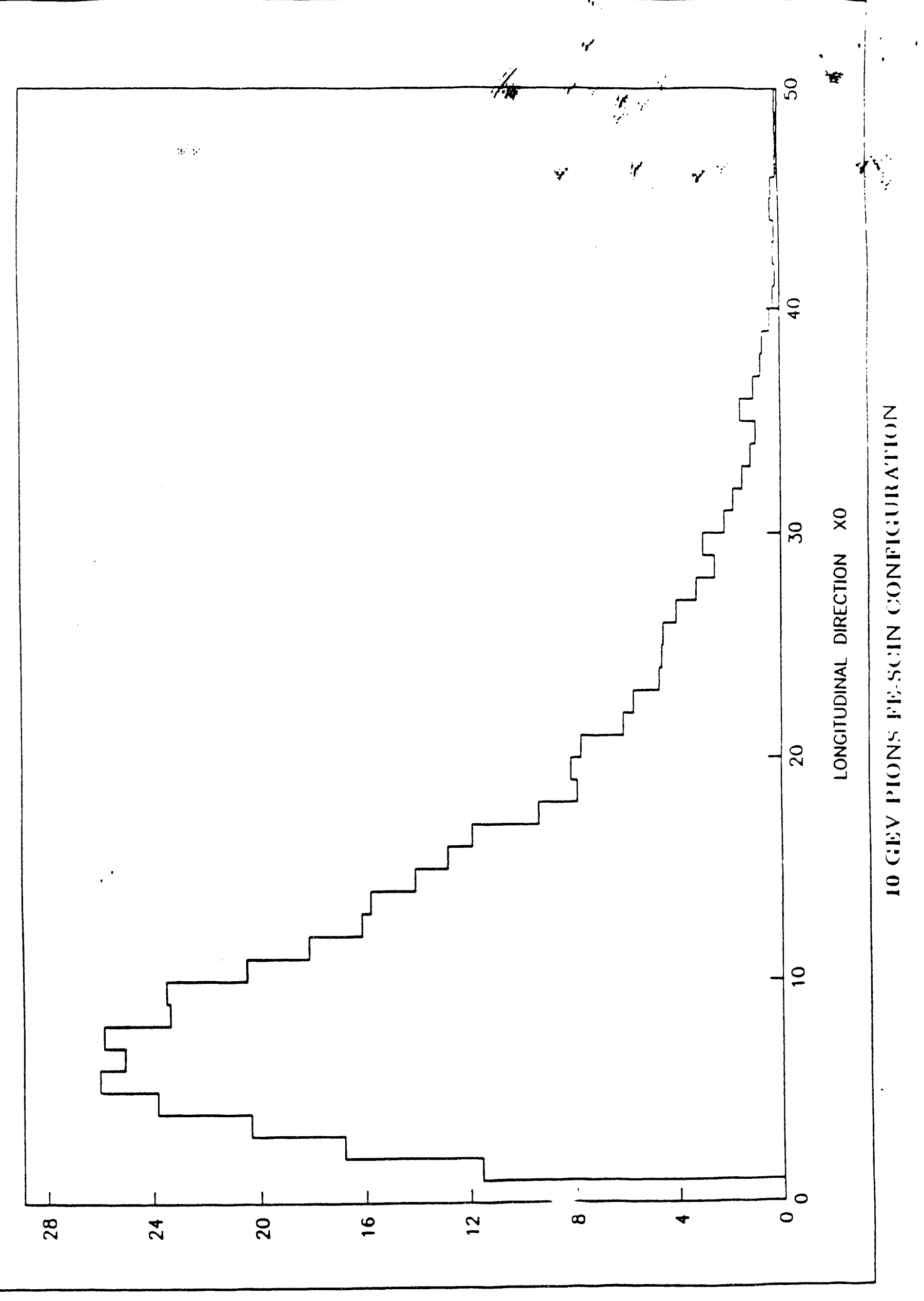




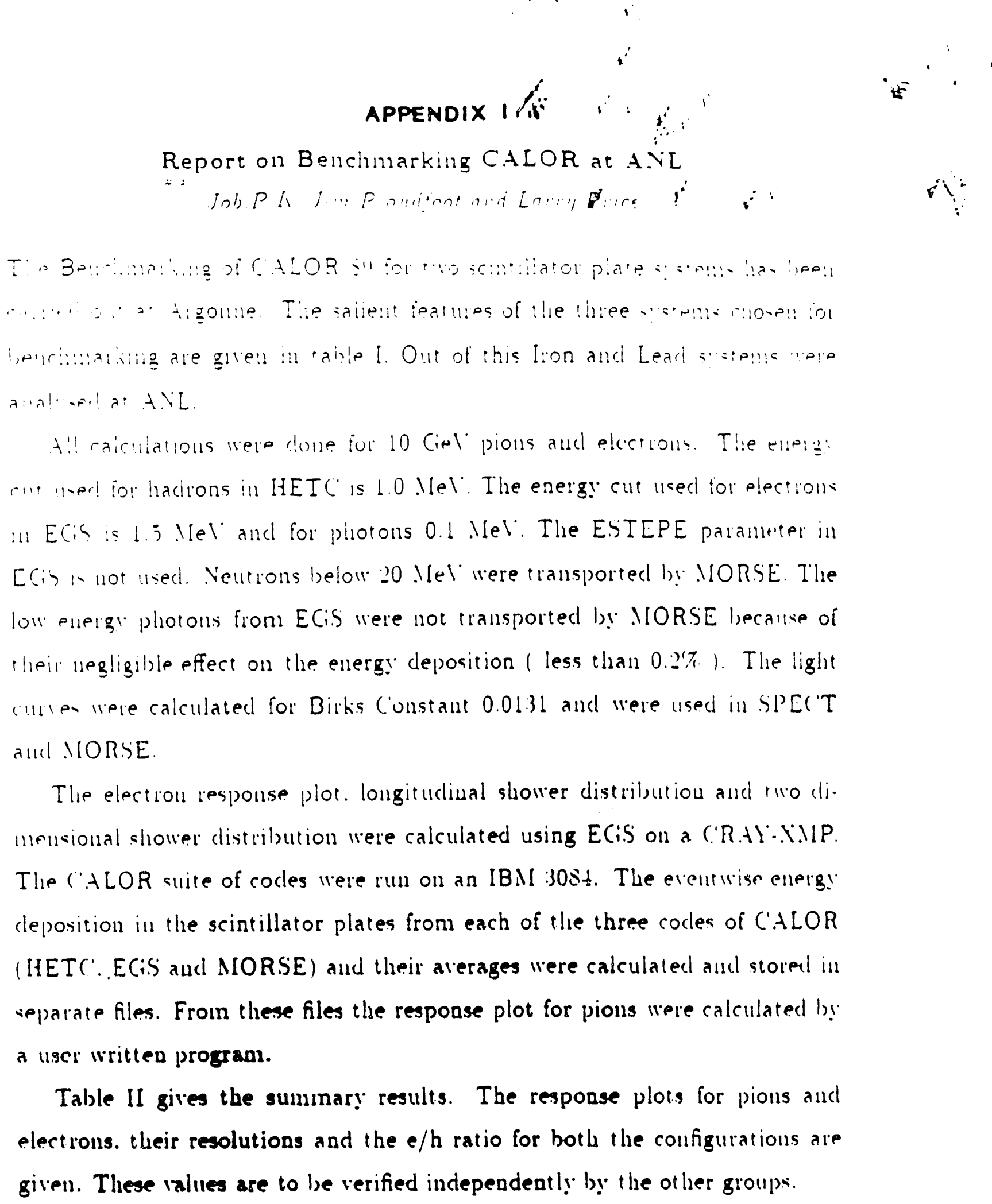




\section{T.ABLEL}

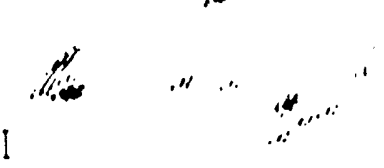

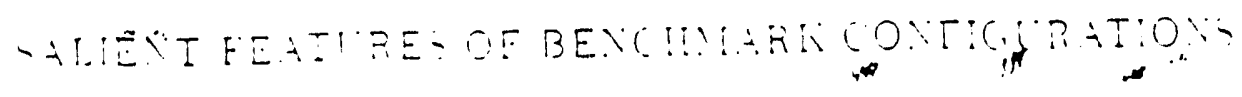

\begin{tabular}{|c|c|c|c|}
\hline & $1, \cdots \cdots$ & $\left.p^{\prime}\right) \ldots .1$. & $=0 .+1 \div$ \\
\hline 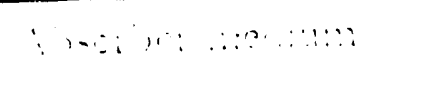 & Don:? Ya:4n & Learl & $i: 20$ \\
\hline 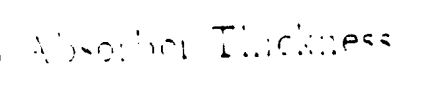 & $03.30 . .1$ & D淀 & $: \quad r:$. \\
\hline 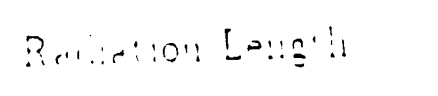 & $1933+\ldots$ & $0.80 \mathrm{c}:: 1$ & $\therefore \therefore$ \\
\hline Vurtear lat ic::og!h & $: 0.5 \mathrm{~cm}$ & $16.70 \mathrm{~cm}$ & $\therefore: 09 \mathrm{cos}$ \\
\hline Darine & $1515 \mathrm{~g}: n \mathrm{~cm}^{3}$ & $11.35 \mathrm{gm} / \mathrm{cm}^{3}$ & Tsi g $\mathrm{m}^{\mathrm{c}} \mathrm{cm}^{2}$ \\
\hline Xnmien Dentil! & $4-1 ! 4 \cdot 10: 2$ & $3.200 \times 10^{22}$ & $S+\leqslant \sigma^{\circ}: 10: 2$ \\
\hline taive medium & Scimtillator & Scintillator & Sciutillator \\
\hline Scmutilaror Thuckues: & $025 \mathrm{~cm}$ & $0.2 j \mathrm{~cm}$ & $0.25 \mathrm{~cm}$ \\
\hline Density. & $1.0+\mathrm{gm} / \mathrm{cm}^{3}$ & $1.01 \mathrm{gm} / \mathrm{cm}^{3}$ & $1.04 \mathrm{gm} / \mathrm{cm}^{3}$ \\
\hline Yimbles Drusit! & & & \\
\hline ('alt,on & $4 . \leqslant 1 * 10: 2$ & $4.1 \times 10^{22}$ & $t . E 1 \times 10^{22}$ \\
\hline Hidiogen & $+1.2=10=2$ & $+\left(y \cdot 2=10^{22}\right.$ & $+.9) \div=10 \div 2$ \\
\hline Birks ('olsslant & 0.0131 & 0.0131 & $0.01: 31$ \\
\hline Cilubal Dimemions & $100^{-1} 100 \mathrm{~cm}^{2}$ & $100 \cdot 100 \mathrm{~cm}^{2}$ & $100 * 100 \mathrm{~cm}^{2}$ \\
\hline Depulh & $\therefore \lambda_{1, \ldots t}$ & $s \lambda_{1 \ldots t}$ & $\leq \lambda_{\ldots}$ \\
\hline
\end{tabular}




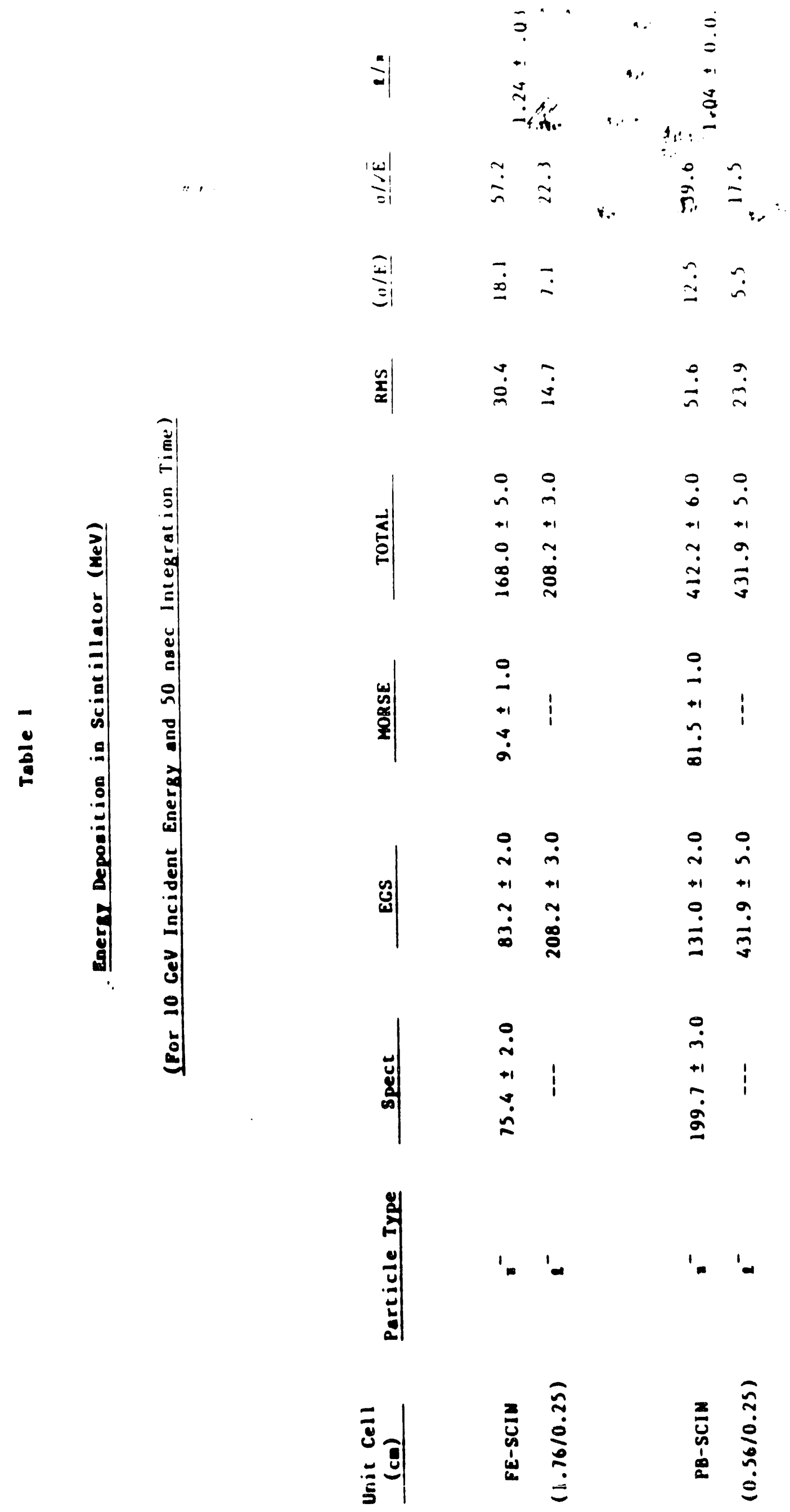




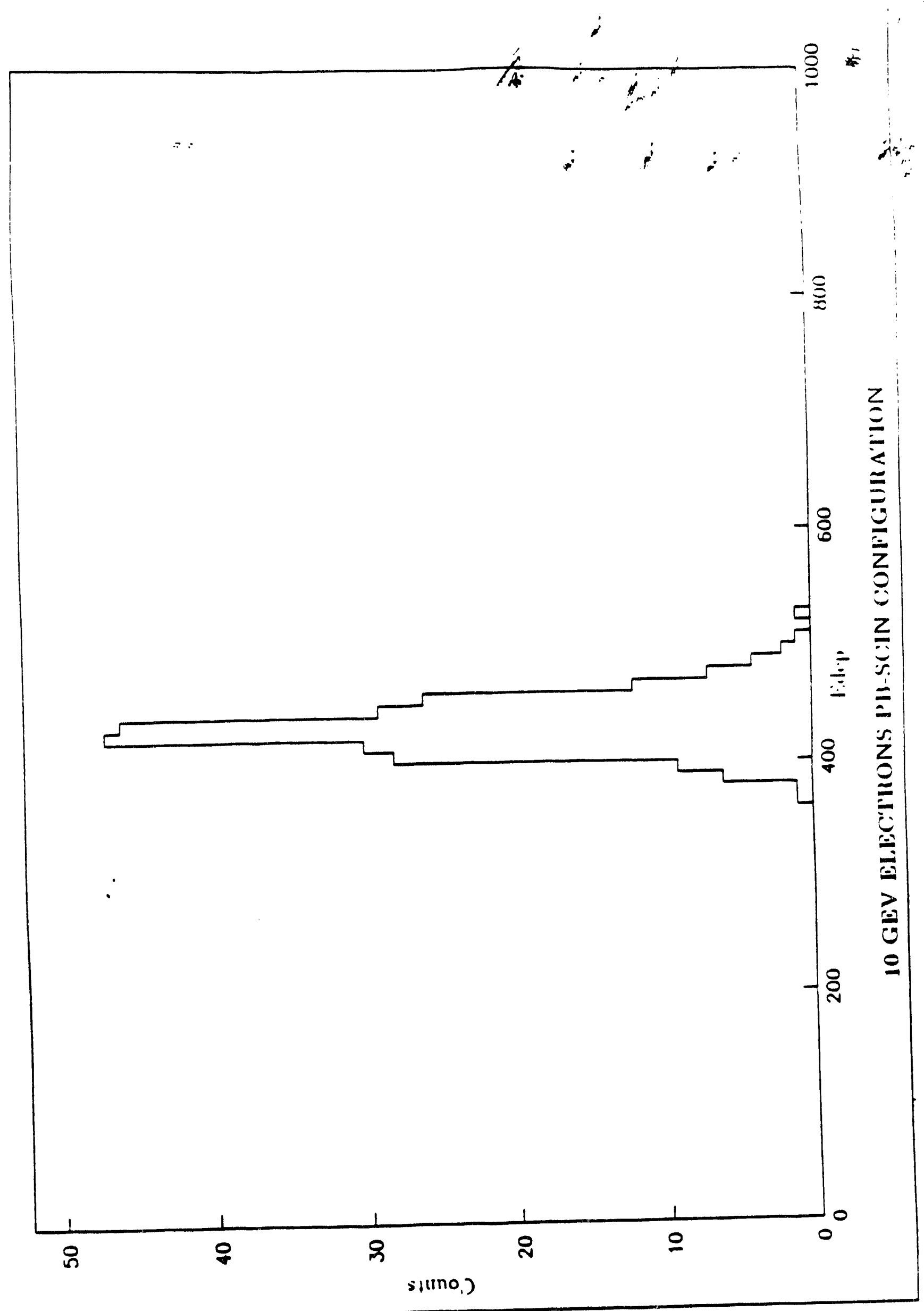




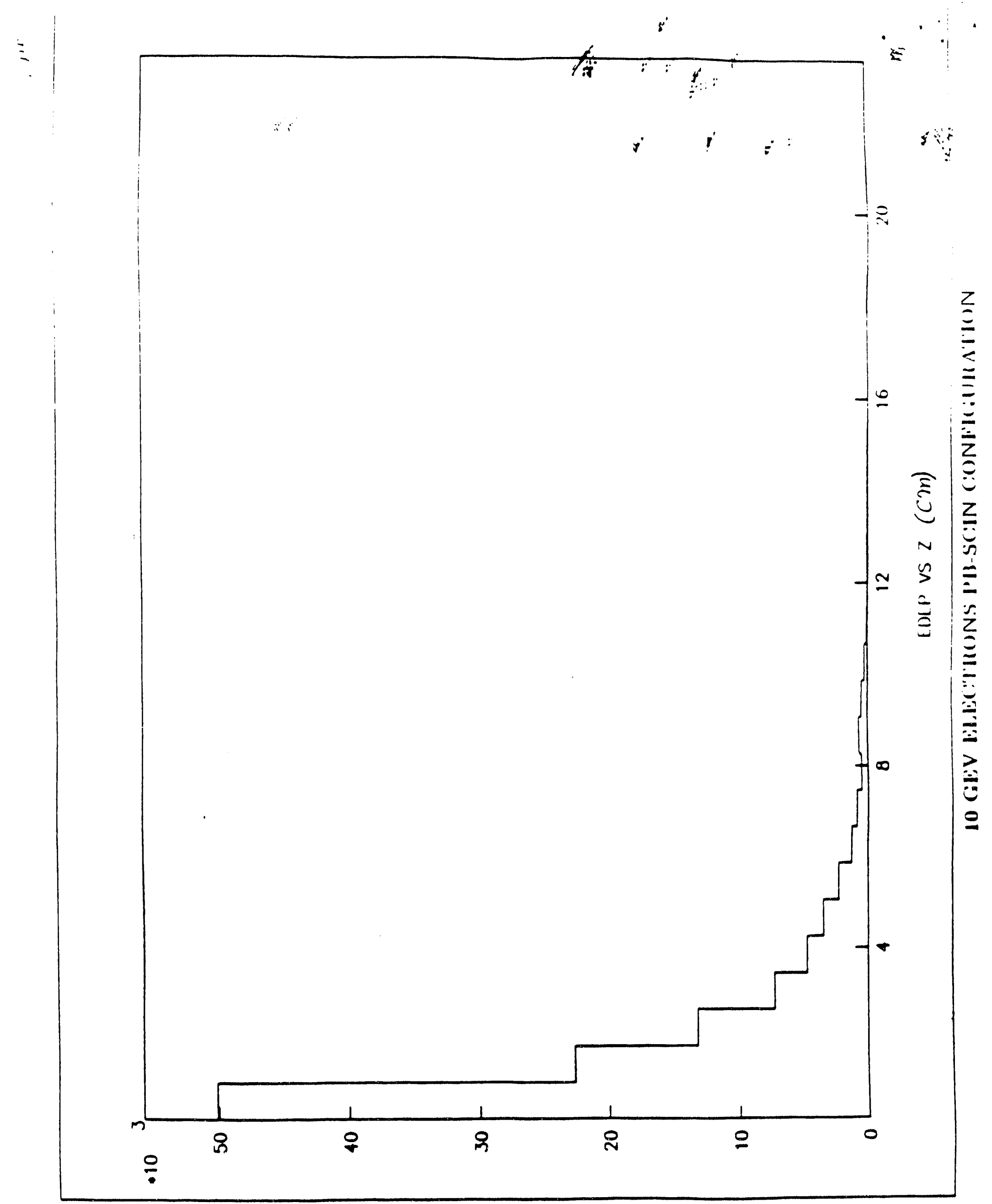




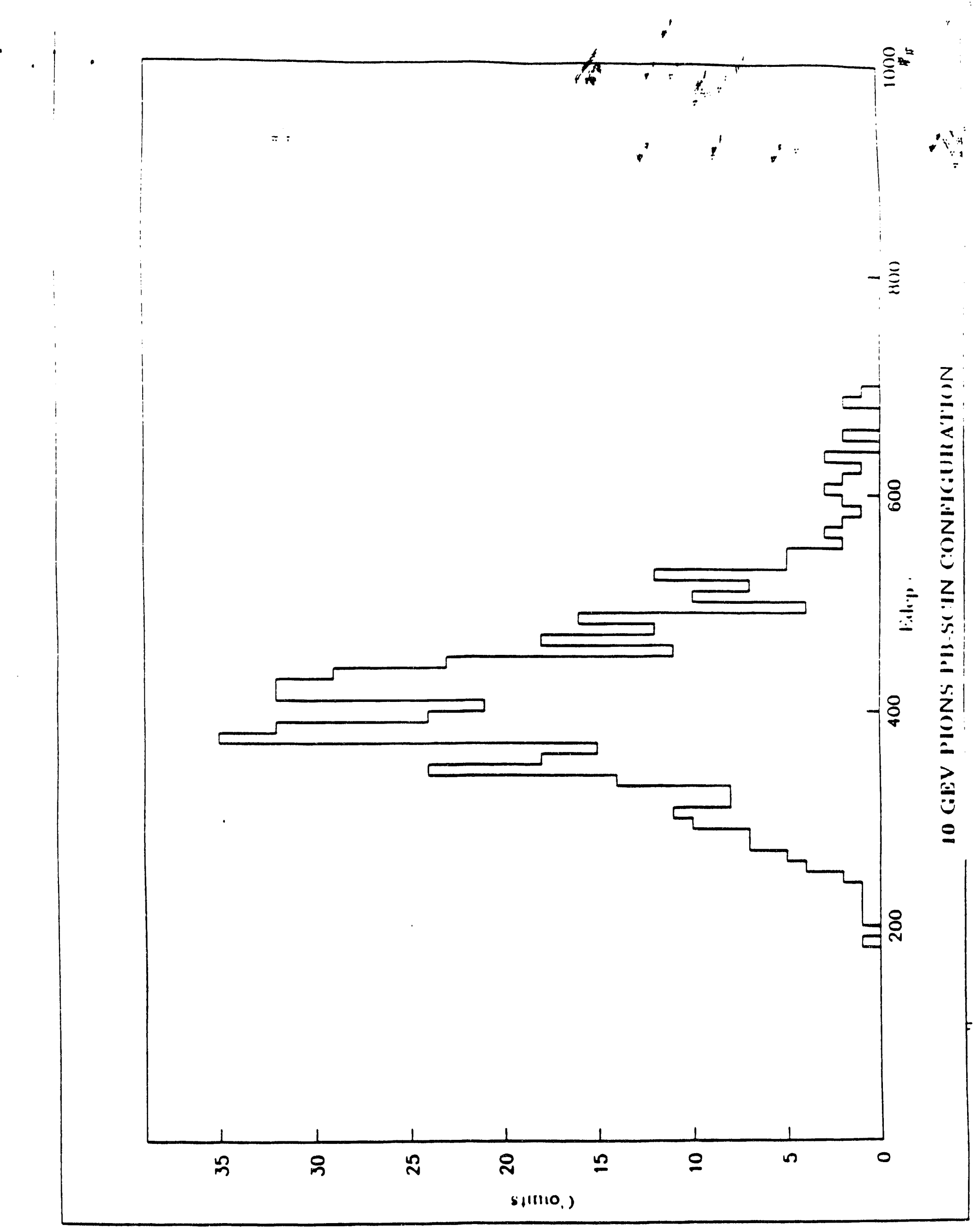




\section{$\stackrel{4}{3} \quad \therefore$}

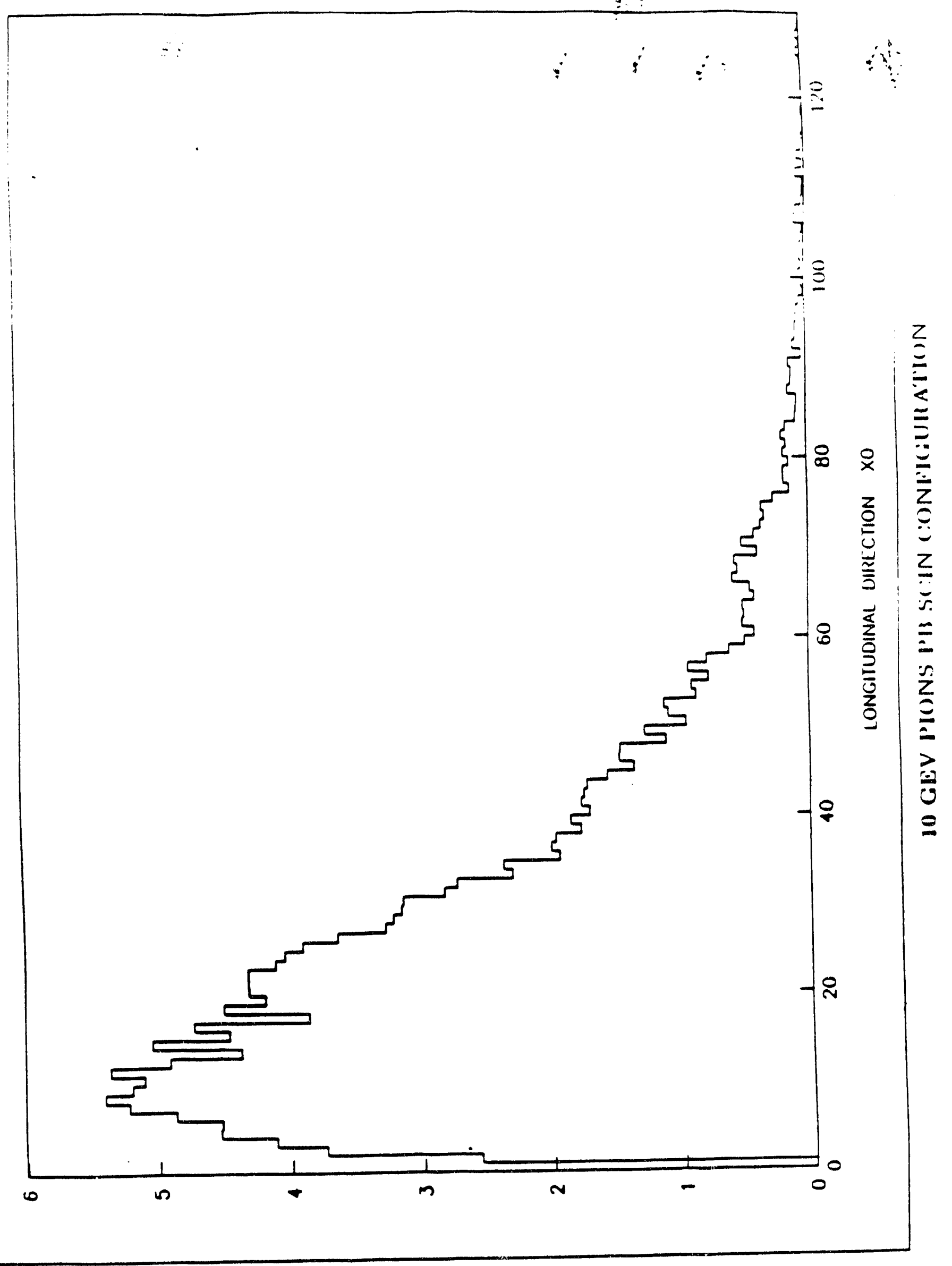




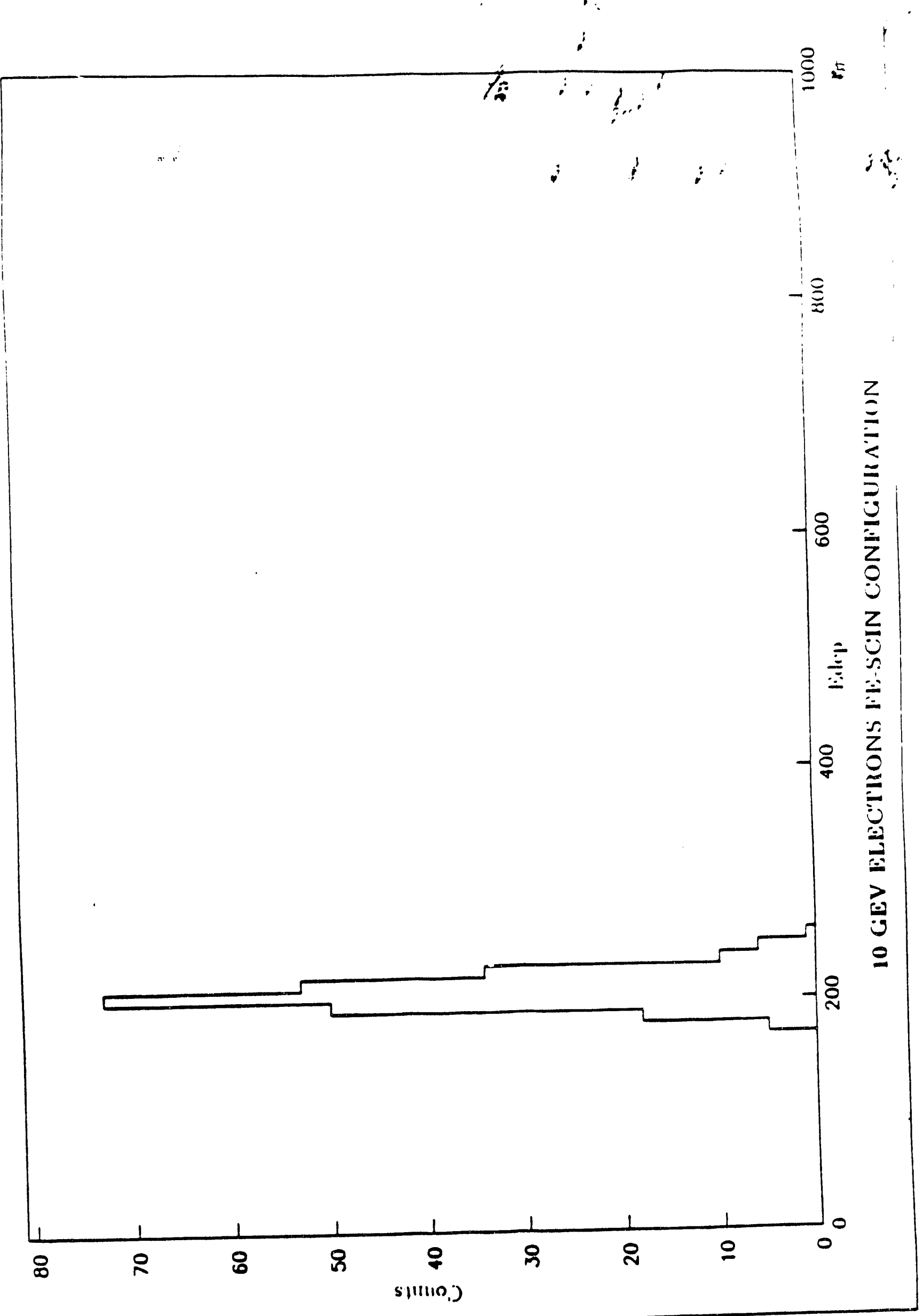




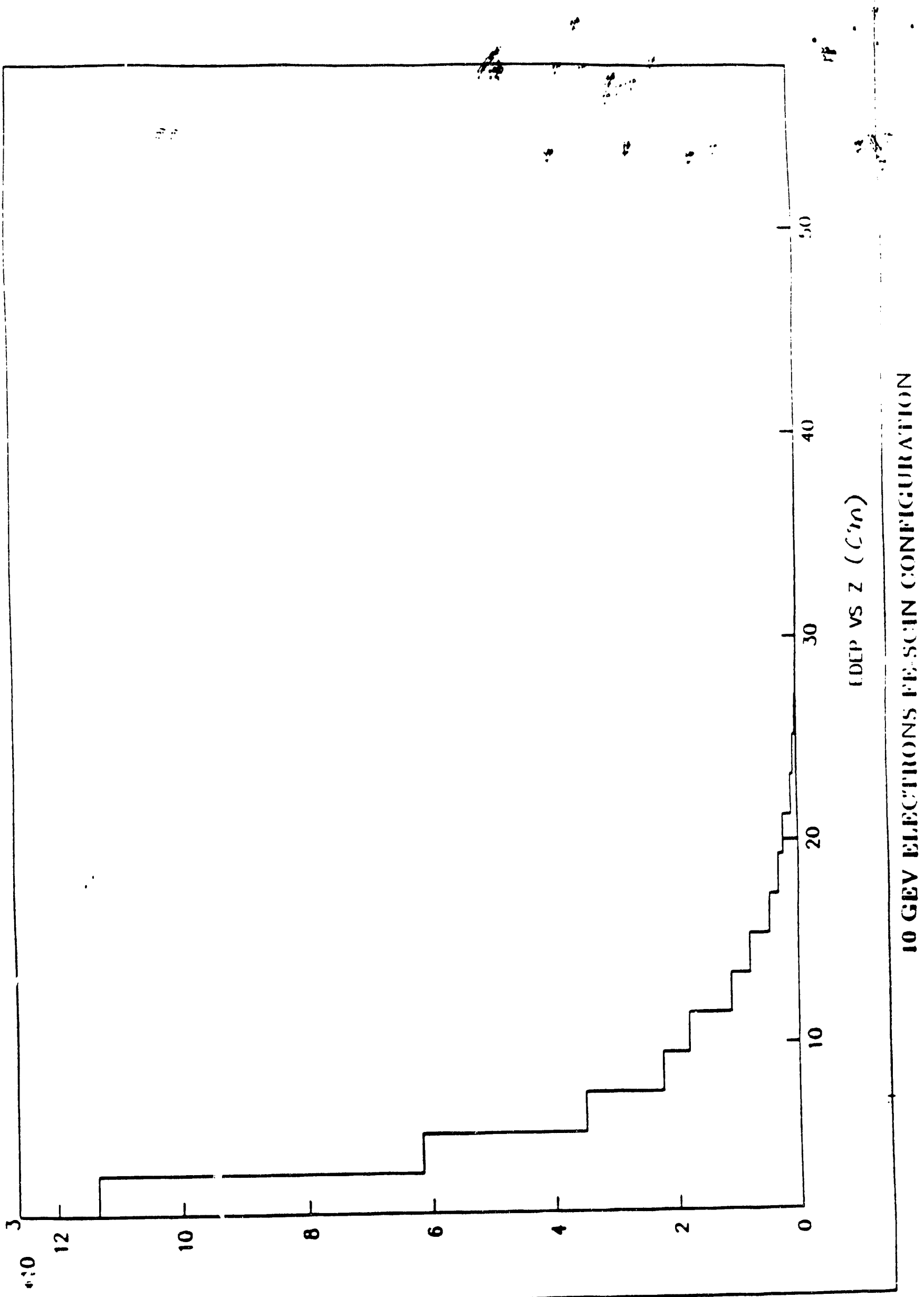




$$
\text { 13 }
$$

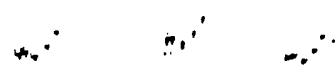

-

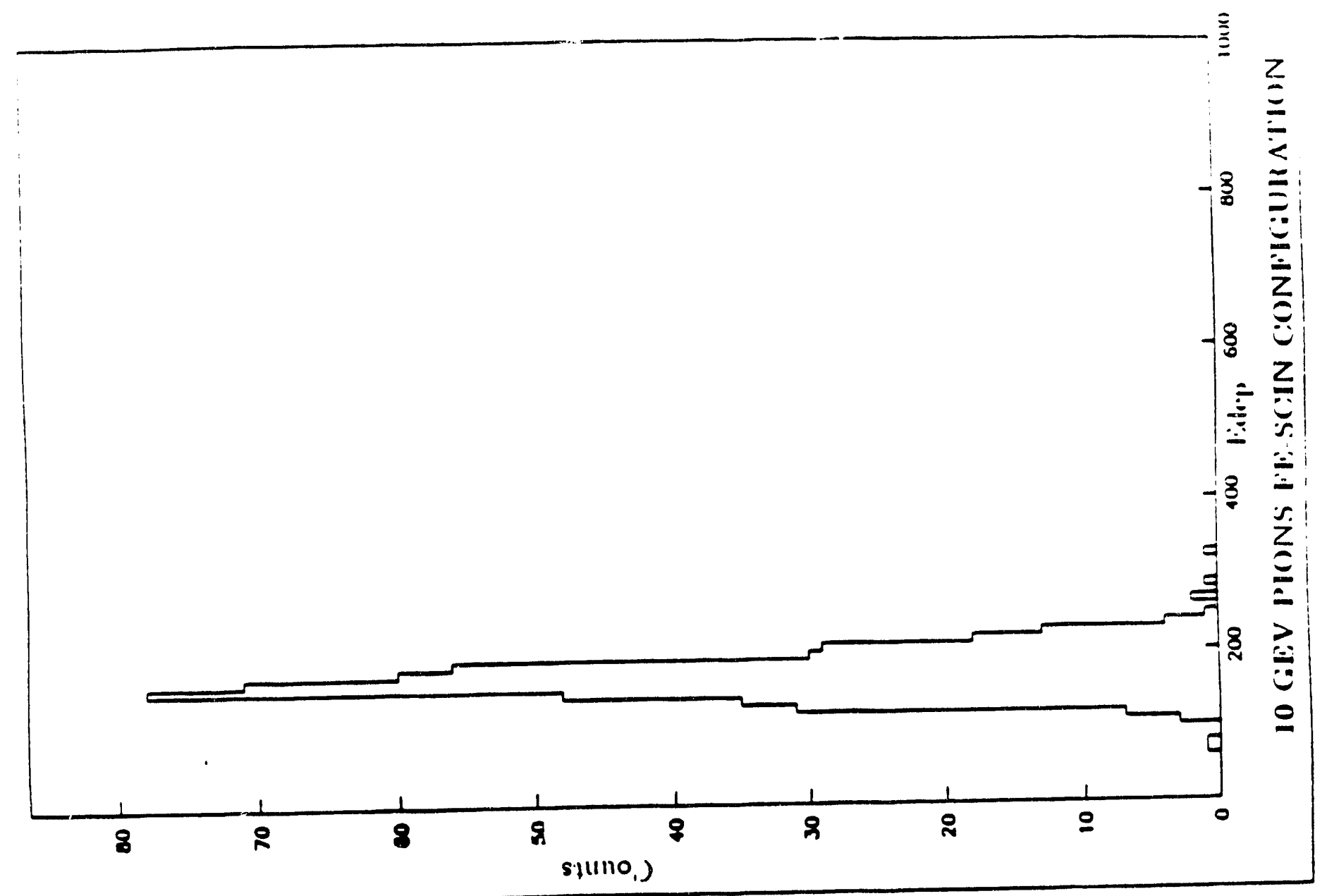




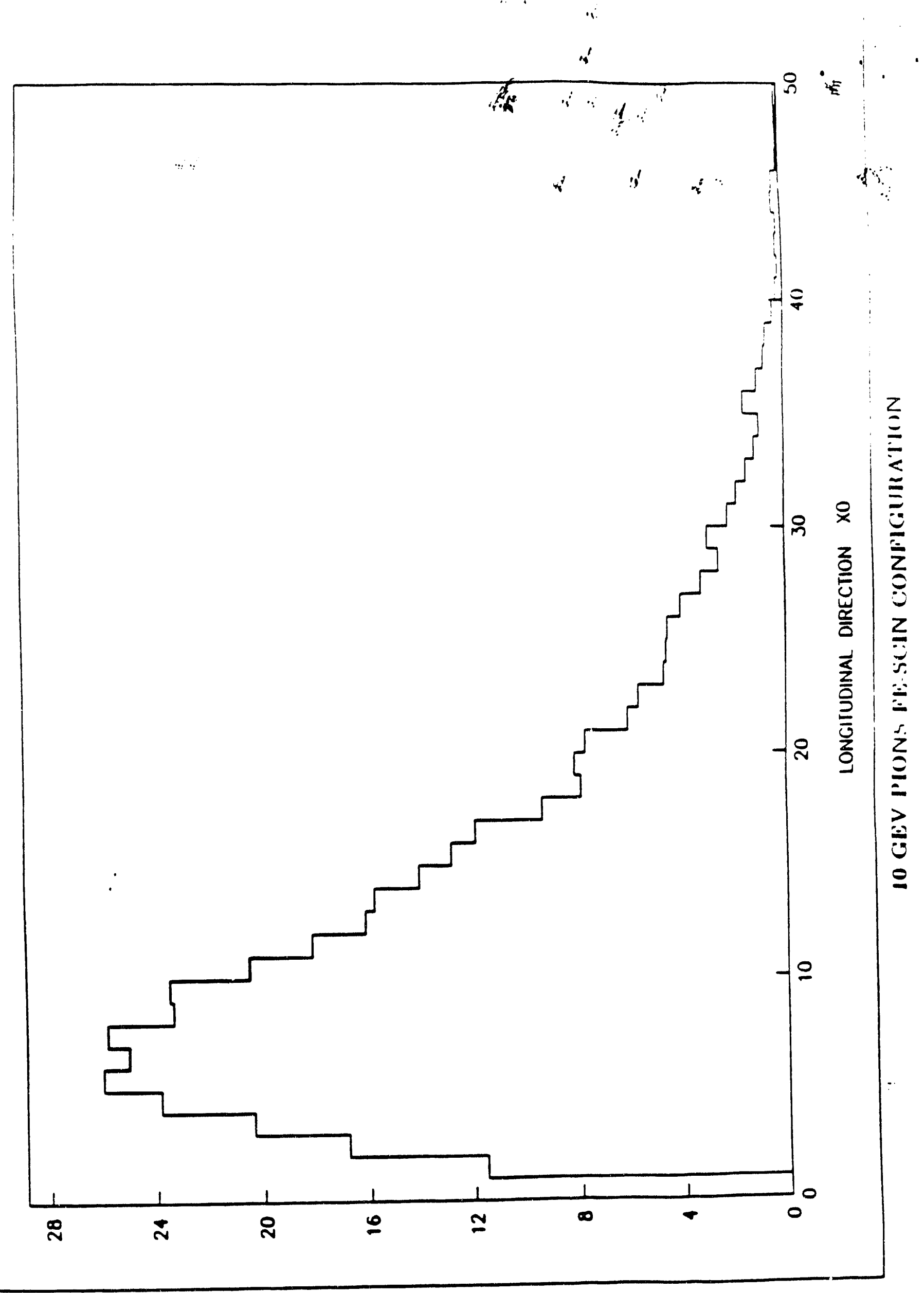




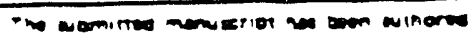
or - coniracior ol on U. 2 Conemmet under Dentract No. W.j1.10e ENC.22

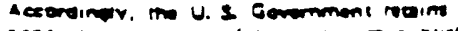

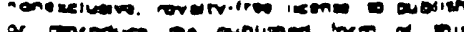
coningution the wollaned orm at nis

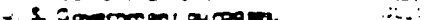

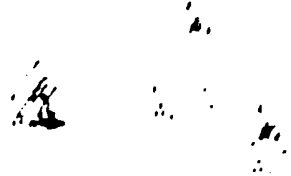

A..II-EER, TR-90-TT

i. SDĆ. $90-000 \div 3$

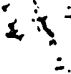

\author{
A First Simulation Study \\ of the Barrel-Endcap Transition Region \\ in a Calorimeter of the Scintillator Tile Design "
}

JAMES PROUdFOOT and HANS-JOCRE. TROST

Eigh Energy Physics Division - 962 .

Argonise National Laboratory

9700 S. Cass Ave., Argonne, IL 60499-1815, OSA

\title{
ABSTRACT
}

We have made a first study of the calorimetric response to $10 \mathrm{GeV} / \mathrm{c}$ charged pions in the transition region between barsel and endeap for the scintillator-tile design pursued at Argonne National Laboratory using the simulation program A.NLSM. For (very nearly) projective tower orientations in the barrel, the crack appears deep within a narrow angular range, causing a loss of the response in that region up to $40 \%$. Pointing the towers onto the beam axis $35 \mathrm{~cm}$ or more away from the nominal-interaction point leads to a shortened deptb of the barrelendeap crack as seen by particles incident from the interaction region, cutting the maximum los down by almont oxe half. The worsening of the resolution follows the same trend. Introduction of a solenoidal coil in front of the calorimeter causes an overall degradation of the response by an amount nearly comparable to the effect of the crack. Dlectrons of the ame incident momentum are more strongly affected by the coil than pions but see only a much narrower region of degradation by the crack.

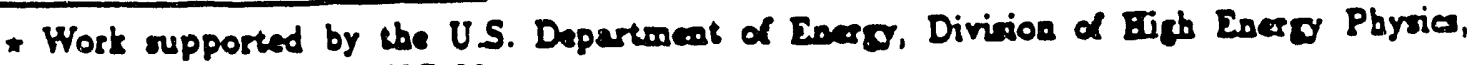
Contract W-31-102ENG-38 


\section{Introduction}

$\therefore \quad \therefore$

Caior:meter designs for SSC detectors are of:en subject to a variety ố muwaly con:aciciory semi-bard requirements and boushary conditions. The undexy:r.g cemards arise fror the physics issues expecied to be acd-essabie by $\subseteq \Xi C$ exper:=en:s. These include bermeticity and bonogeneity of the response over most of the solid angle and requirements on position and energy resolutions for electromagnetic and hadronic showers. In particular at the size scale to be consicered at the SSC, the techaical feasibility tends to create contradictions between those requirements. For instance, good spatial resolution is best achieved with a projective-tower readout geometry. This leads either to projective gaps ruining the bermeticity and local homogeneity of the response or to large cracks between large modules or to large amounts of nonsensitive materials in the region of the shower development.

In this note we present a simulation study of the barrel-endcap transition region for a calorimeter design as pursued at Argonne National Laboratory for a scintillator-tile sampling calorimeter." In the following, we describe the tools and procedures used (section 2) and the results of the simulation runs and conclusions (section 3).

\section{Tools and Procedures}

The starting point for the present study is a design by N.Hill ${ }^{(1)}$ shown in Fig.1. We have coded this geometry as shown in Fig.2 for use in the simulation program ANLSIM, ${ }^{(2)}$ which is based on GEANT? ${ }^{(3)}$ In the aximuthal direction, the setup is simplified for the simulation a being cylindrical everywbere. The tower geometry is coded and handled independently of the GEANT data structures. The simulation program is kept fexible with respect to the deaign by basing it on a number of parameters measuring various dimensions that can be set at runtime. Thus a design change can be accommodated without additional coding 


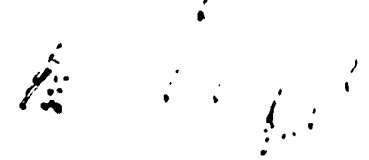

and recompilation, within sore limits. The relevant parameters are listed in Table 1. In this setip, the coil produces a bocogeceousjaxial ragnetic Eeid cई 2 I streagth (dialed by input at run time) is ibe volure it eacioses. There

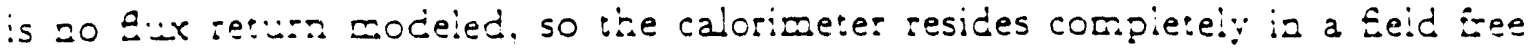
reg:on. The esser:ial ieatures of this design for ite present study are detailed : Appeadix I whici nay serve as a guide to set the input variables for the progra= as riei.

A set of options are investigated below making use of the selections offered in the program setup:

1. the standard design as given by N.Hill, with 7 towers at the center oriented straight at a right angle to the beam axis, i.e. 3.5 on each side of $z=$ $0 \mathrm{~m}$, four more towers maintaining the radial thickness of the barrel and pointing, like aلl further barsel towers, to $z=3.5$ towerwidth, and endcaps "Etting" into the conical end faces of the barsel with axially oriented towers of constant cross section; this is a nearly projective geometry in the barrel, avoiding the gaps between the barrel and the endcaps to point directly to the interaction point;

2. the strictly projective design, different from the standard simply by having no straight central towers;

3. further nonprojective designs like the standard one with the offset of 3.5 tower widths replaced by $1.5,2,2.5,3,5.5,7.5$ and 20 tower widths.

The variation the offet for projecting the towers determines the projectivity of the crack between the barsel and the endeap. In all cases, the endeap is rnoved in to just touch the aluminium support cones ( 1 inch thick) of the barrel. This leads to a minimal air sap. No muterial inside the rolume enclosed by the calorimeter is simulated in the round through varying tower offets; the magnetic feld in that volume is taken present nevertheless. The calorimeter response is calibrated in the simulation for electromagetic and hadronic ener $y$ deponitions separately to recombine to an e/ $\pi$ ratio of close to 1.0 and to reproduce the absolute energy 
scale, both to withig about three percent.

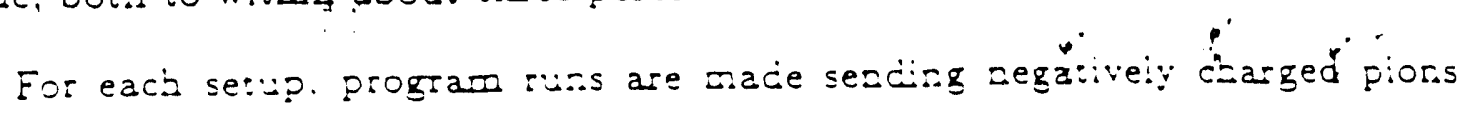
of Foreatum $10 \mathrm{Gel} / \mathrm{c}$ into the caiorimeter at 14 vaives of pseudorapidi: $\eta=1.20$. : $30: 34-1.53$ in steps of 0.04 , at 1.80 and inierzediate pois:s a: 1.40-1.52. and randomiy crosen azimutb 0 . The center of the gap betreen $\therefore$ be bar:e! and the endcap is located around $\eta=1.44$. The pion induced stowe:s are simulated in A.VISIM using full analog simulation with rela:: rely high cuis on the kinetic energies; they are set to $100 \mathrm{MeV}$ to limit the execution times. This will misestimate the absolute resolution somewhat and also slightly reduce the shower size. It will nevertheless give a reasonable relative comparison of the design variants under consideration.

\section{Results on Calorimeter Response}

The total deposited energy before any clustering is performed is taken as the response of the calorimeter for the present purposes. The distributions of this energy normally contain about one thousand entries for each preset value of the pseudorapidity 7 . The results for three of the offsets investigated are collected in Table 2.

The calorimeter shows a lat response (Fig.3) of about $10 \mathrm{GeV}$ with a deep dip of observable width in the gap between the barrel and the endcap developing as the tower orientation ofiaet goes towards zero. (The error bars for the means in this figure show the r.m.s. value of the distribution and not the error of the mean, for the r.m.s. values, the error bars bave no physical meaning at all.) At the "standard" decign offet of $35 \mathrm{~cm}$, the dip is a little more than $20 \%$ for $10 \mathrm{GeV} / \mathrm{c}^{-}$incident, up from $40 \%$ for the projective devign, and has begun to level off in dependence on that offet (Fig.4). Adding more material in the gap will widen and deepen the dip in the response curve particularly for (nearly) projective designs. Choosing a point awa trom the deep-dip region as given by 
the present study will then :roride some safery raigin against getting into lhe

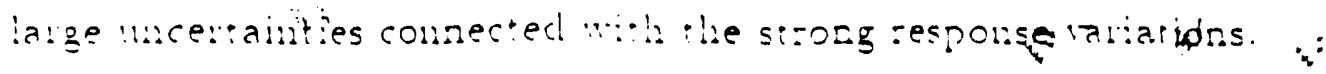

iTe then inclucle a sinple :aociel of the solenouclal coil in sont of the caluri:ue-

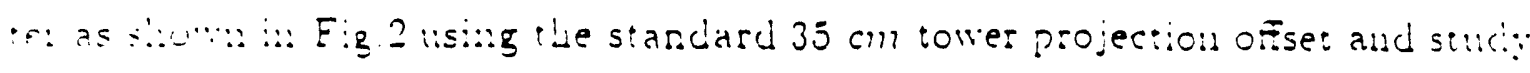

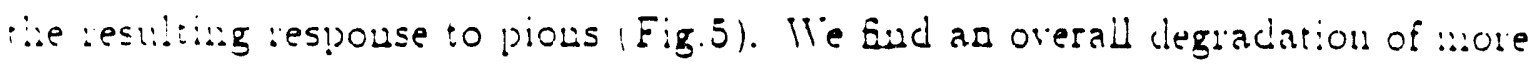
:han $10 \%$ witha the solich ang!e corevage of the coil nith only baif of that effect in : he crach-related dip. Clear!y, the coil as modeled prorides a scatteniug centr in fint of the crack that spieacls the energy deposition beyoud the coil into the censitive tegions of the calorimeter. The range in pseudorapiclity affected by the coil eud is about $\eta=1.42-1.52$ and the resolution is degracled by less than $30 \%$. This is celtainly a somen hat too optimistic picture of the reality since there may" be wore unaterial in the coil end itself ( $\pi$ e use a massive aluanium tiug of thich: neas $15 \mathrm{~cm}$ along the beam a.نis and $30 \mathrm{~cm}$ sacliall! ) and nill be more matesial for support of tracking systens and supplies for evergtbing inside the voluue enched by the calorimeter. For comparison. the simulation efforts for the SDC' Expression of Interest ${ }^{|0|}$ assume the range of degradation to be $\eta=1.25-1.5$ aud the increase of the resolution up to $70 \%$.

An aclclitional chech on the performance for the stauclard geometrical choice is to look at the response and acceptance for electrons. Wie performed ruws fur the stauclard desigu nithout and with coil in place for electrons of $10 \mathrm{Cicl} / \mathrm{c}$ usomentum. For the set and deusity of points in pseudorapidity used. the crack produces a rery deep but also narror $\left(\Delta_{\eta} \approx 0.01\right.$ mide $)$ dip in the response. The cuil takes much more energ ariag foom the electrous than from pions as has to be expected (Fig.6) and also rorseus the respouse in the dip significautly. This means the design under consideration essentially will have a uarrow ring aluost blinel to electrons of moderate energies. This is probably acceptable unless the gap has to be widened for hardware support iustallations a lot.

To couclucle, we hare obtained support from full simulations of pion shoriers for a prefereuce of using a quite moderately nonprojective tower genunetry in thr 


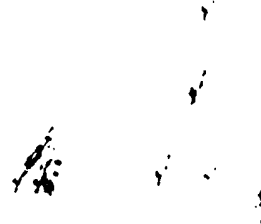

$r:$

absorber-scintillator tile calorimeter as considered for SDC. The tower orientation cetermines the orientation of the gaps in the calorimeter coverage, apd it is these gaps inat bave to be well covered by acive detectors is a view from the interaction. point. The impact of having a solezoidal coil in frox: of the barel calorinete: $\therefore$ : exds of xisich reach into the barel-endcap region is in size comparabie to the effect of the crack itself. In the finai detector, the crack will cortain more materiaj than only the support cones for the barrel calorimeter. For that total arount of passive material, the 1 inch tbickness of the support cones is a very crude and possibly thin approximation. In continuing this study, we will look at the impact on particle and jet reconstruction in some detail, and we will also atternpt to improve the geometry to reflect more realistically the dead material needed by the detectors and the simulation techoique to study bigher particle energies. 


\section{APPENDIX 1}

The following is a detailed description of the structure of the geometry setup

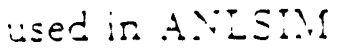

1. The central cowers in the barel are oriented strictly at a rigin angle $x: 1:=$ respec: to the bean axis, thus they are not projective in the ( $r, z)$ piare (where $r$ denotes the radius in the plane transverse to the bean axis). The number of these towers, counted from $z=0$ to one side only, is given in ise variable BARTOF. In the range of these towers the total transverse thickness of the barrel calorimeter is set by the variable BARDRC. The side length of the central towers at their font face is taken to be BARTOH.

2. At the front faces, a conical shell of aluminium of thickness BARCOM is attached to support the barrel.

3. By projecting the outside boundary of the central towers onto the beam axis, a secondary origin is defined. From this origin outward, i.e. away from the main coordinate origin, a pseudorapidity variable is used to define the polar boundaries of the remaining barrel towers. A constant stepping in pseudorapidity produces a constant projected size of the towers at the inner surface of the barrel (see appendix 2). The same side length BARTOH is imposed here for the projected size that is used for the central towers also. Thus, a strictly projective tower geometry in the barrel can be produced by having zero central towers as defined above; this is in fact allowed by the simulation program. In turn, an extremely nonprojective geometry can be defined by making the central towers extend over most of the barrel range. This range or half length at the inside surface is defined as the balf length of the coil COIL28 plus en extension BAREXT over this point, truncated to the largest number of complete towers that cas be contained in this bound.

4. Across the first BLRTOE towers in the - relative to the secondary origin - projective region, the central transverse thickness of the calorimeter is 


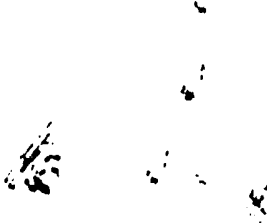

maintained. Beyond those, the outer transverse racius decteases lisearly with the axial "coordinate $z$ to produce an outer sicle leng:L ZARDRE pf the "lasi" ring of barrel towers. (That leag:b is measured on a ray from the secondary origin along the sice iace of the towers.)

5. The ezdcaps are construciec as thick cyinders (leag:b/depti IYcuzC, ra. dial exient EYDORT starting at an inoer radius EXDRIN), with a (ew (EYIDR:) rings within the radial exient reduced in depth on the inside and a large: number (ENDNST) of rings added on the outside to form an approxination to a conical shape. The inside rings follow a cone with its apex at the primary origin. The outer rings are aligned to bave their edges at a constant distance from the outermost ring of barrel towers, i.e. they follow a cone parallel to the cone with an apex slightly further out than the secondary origin and an angle given by the end face of the barrel. The placement of the whole endcaps aling the bearn axis is governed by the half length COIL2E of the coil and the gap width ENDGAP between the coil and the front face of the endcap. The towers are oriented axially, i.e. not projective; this feature is fxed.

6. The outer rings of the endcaps are divided into a short and a long remnant piece each such that the short rings just overlap by a given amount EIDRGO. The idea is to allow to form pseudotowers on the endeap side of the gap between the barrel and the endeap that approximately continue the tower structure of the barrel by one more layer. This could be useful for pattern recognition acroos that gap. In the present study, this feature has not been used and the short pieces are effectively joint with the longer remanants into one piece.

7. The azimuthal subdivision troughout all of the calorimeter parts is chosen to give a width at the inner face of the calorimeter as close to the desired tower side length (BARTOW and ETDTOH reop.) a possible under the restriction of having an integral number of towers around the aximuthal 
circumference. (This wiil gi $:$ e an inaccura:e =odel of the periornarce of

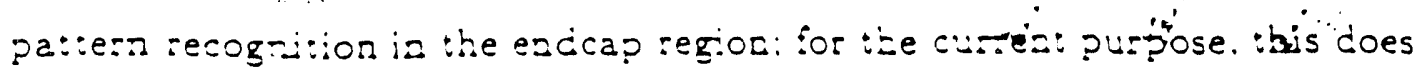
.ot mat:er.)

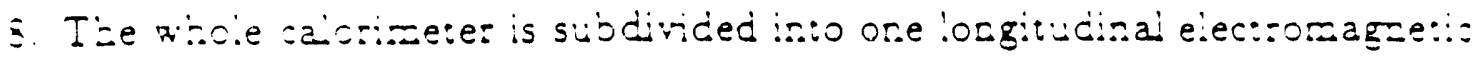
seci:ion of :ransverse/axial thickness BARSEY and EXDDEM res?. arci ose hadronic sec:ion.

9. Each tower (electromagnetic and badronic separately) is assigned artinciaiiy a coordinate in the pseudorapidity-azimuth plane $(\eta, \phi)$ as follows:

(a) The $\Phi$ coordinate is taken at the azimuthal center of the tower in all cases.

(b) The $\eta$ coordinate for the electromagnetic towers is taken at the balfway point into the tower on theis center lines.

(c) The $\eta$ coordinate for the hadronic towers in the barrel and in the main body of the endcap is taken 1.5 times the depth of the electromagnetic section (not the length of the tower in front), i.e. BARDEY and ENDDEM resp., into the badronic section at right angles, not measured along the center lines of the towers.

(d) In the rings of the eadcaps, the assigned eta coordinates are picked on cones connecting to the plane used in the main body, with a cone angle taken wide enough to conserve the spatial radial order of rings of towers in the peeudorapidity order.

10. The detector geometry can be extended (vis input lags at run time) to include a beam pipe, a coil, a model of an all-scintillating-fiber tracker, and a muon system. The coil parameters are used in the setup of the calorimeter as is evideat above; they are available and modifiable even if no actual placement of the coil into the geometry setup to be known by ANLSDM/GEANT is requested. 
The routines needed to define and use the setup are kept in a separate Ele. repiaci=g the default ones of A.VISIM at linking time.

\section{APPENDIK 2}

Ihe equivalence of constan: tower size in the pseudorapidity coordinate $\eta$ and :s projec:ed size (side lengrb $2 \cdot \Delta w$ ) at the inside of a cylinder of inner radius Ro can be seen as follows:

Let the ray from the interaction point into the center of a tower bave the polar angle $\Theta$ with respect to the beam axis. Then the balf width $\Delta w$ of the tower is related to a deviation of the polar angle

$$
\Delta w=\frac{R_{0}}{\sin (\theta)} \tan (\Delta \theta) .
$$

In order to study the angular dependence of the "cumulated half widths" function $w$, let us use in the limit of small angular deviations the approximation of the tangent function by its argument,

$$
\tan (\Delta \theta) \rightarrow \Delta \theta
$$

This substitution leaves the limit $\Delta \theta \rightarrow 0$ of $\Delta w$ invariant, being

$$
\frac{d w}{d \theta}=\frac{R_{0}}{\sin (\theta)},
$$

as the difference $\tan (\Delta \theta)-\Delta \theta$ is continuously differentiable in a whole neighborbood of zero and it and its first derivative vanish at zero (the former quadrat. ically as its Taylor series exists and converges in that region). By integration trom $\theta=\pi / 2$ to any polar ancle we find ${ }^{\text {th }}$ for the function $v$ the representation

$$
w(\theta)=R_{0} \ln \left(\tan \left(\frac{\theta}{2}\right)\right)=-R_{0} \eta \text {. }
$$

Thus, the request arising from the physics of jets to define towers with constant width on the (pseudo) rapidity scale and that one arising from pattern recognition to define towers of constant widtb scroas the lateral extension of showers 


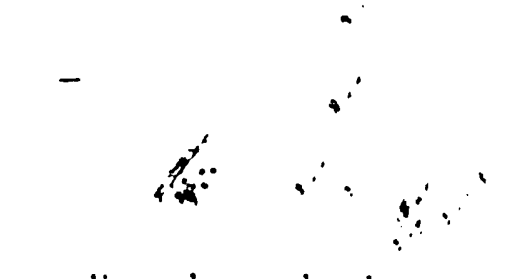

actually coincide in a cylindrical calorimeter aligned on the beam axis. This coincidence is lost in the endcaps where $\Delta w$ bas a different dependence on $\theta$. 


\section{REFERENCES}

2) X.H:11. Observations and cocciusions drawn from oonceptral design exe:cises on barrel calorimete for SDC. internal note, Argonne National Laboratory. . Vay 30. 1990 (uspublished)

2) R.E.Blair, L.E.Price and H.J.Trost, program A.VLSLM, Argonne National Laboratory, Argolne, L1: ais, USA 1989, version 1.04, 1990, ur. publisbed:

F.-J.Trost, talk given at: Worksbop on the Future Development of GEANI, SSC Laboratory, Dallas, Texas, Jas.10-13, 1990, unpublisbed; R.E.Blair, in: Workshop on Pbysics and Detector Simulation ior SSC Experiments, SSC Laboratory, $\Gamma$ llas, Texas, Jan.9-19, 1990, vol.1, p.69; H.-J.Trost, ibid., vol.2, p.255

3) R.Brun et al., GEA.NT 3, CERN DD/EE/841, version 3.13, CERN, Geneva, Switzerland 1990

4) Solenoid Detector Collabolation, Expression of Interest to Construct and Operate a Detector at the SSC, May 24, 1990

5) 5.N.Bronshtein and K.A.Semendyayev, Bandbook of Mathematics, 3rd English edition, Van Nostrand Reinhold Company, New York, New York, USA 1985 , section 1.1 .3 


\section{.}

\section{TABLE CAPTIONS}

1. Design paraneters used in the geometry-setup for A.VISiMl (engths in cm: detallts in parentheses)

2. Pseudorapidiy polar angle, momentum components, mean response and r.m.s. of the response for a) the standard design, b) the projective design. c) the bighly zonprojective design with $2 \mathrm{~m}$ offset

\section{FIGLRE CAPTIONS}

1. Site view of the calorimeter design addressed in this study

2. Side view of the mechanical calorimeter geometry as seen by the A.VI. SM program - standard design with optional coil

3. Mean response and resolution for $10 \mathrm{GeV} / \mathrm{C}^{-}$of the calorimeter of ref. 1 for different offsets of the tower orientation; the errors on the mean shown are the resolutions, and the error bars shown for the resolution have no physical meaning

4. Minimum response to $10 \mathrm{GeV} / \mathrm{C}^{-}$in dependence on the tower projection offset; the additional star for $35 \mathrm{~cm}$ offet shows the effect of the solenoidal coil.

3. Mear response and resolution for $10 \mathrm{GeV} / \mathrm{C}^{-}$of the calorimeter of ref.1 without and with coil in place

6. Mean response and recolution for $10 \mathrm{GeV} / \mathrm{Ce}^{-}$of the calorimeter of ref.1 without and with coil in place 
FEzE- ZN

픈

20:Z:ZY

ca:- 5.5

c=…

ㄷ..

Сニ:-EY

ca-ou:

C::-2 2

ここ:Lこ3

EAREX:

BARGAP

QARERC

BARTOF

BARTOE

BARDRE

BARDEY

BARTOH

BARCOA

EIDGAP

EIDRIX

E.TDDRT

EXDDRI

EMDDZI

E.DDZC

EXDDEx

E.TDRO

EDTOH

EIDIST

Tabie:

number of additional tovers beond the constant vidtb ones for vbich the radial tbickness of the barzel renains constant (4.0)

total doptb of barzel along side to and cap (243.8)

thichess of electromagetic barrel section $(19.3=7.592 \div 2.54)$

projected azimutbal front vidtb of torers (10.0) thictesese of alanigin support cone at end faces (2.5)

space betreen coil and and front saca of and cap (25.0)

ianer radius at froat lace of and eap (50.0)

radial thichess of and cap at froot face (180.0)

radtal nember of laner tovers oborter than full artal thletoses of and eap (3.0)

langth of lnaernost hadronie andeap tovers (12.8) axtal thictoses of and cap (278.2-375*0.292*2.54) thichase of electronametic and cap rection $(19.3=7.592 \div 2.54)$

arial orerlap of rlage $(2.3=3 * 0.292 * 2.54)$

ide langth of and cap tover (10.0)

nuber of etepe for outalde rings of and cape (11) 
Table 2a

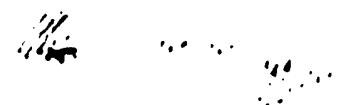

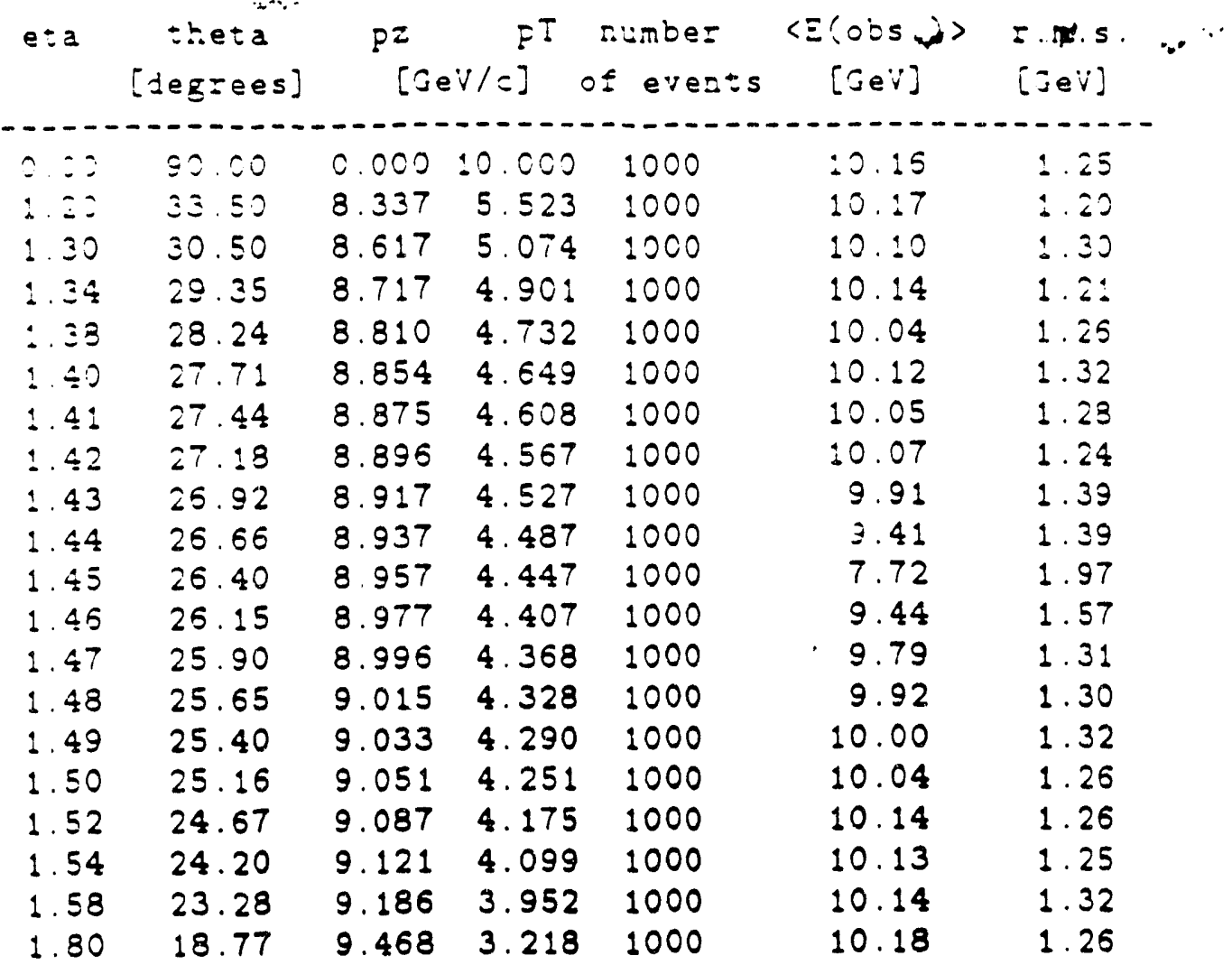




\section{Table 2b}

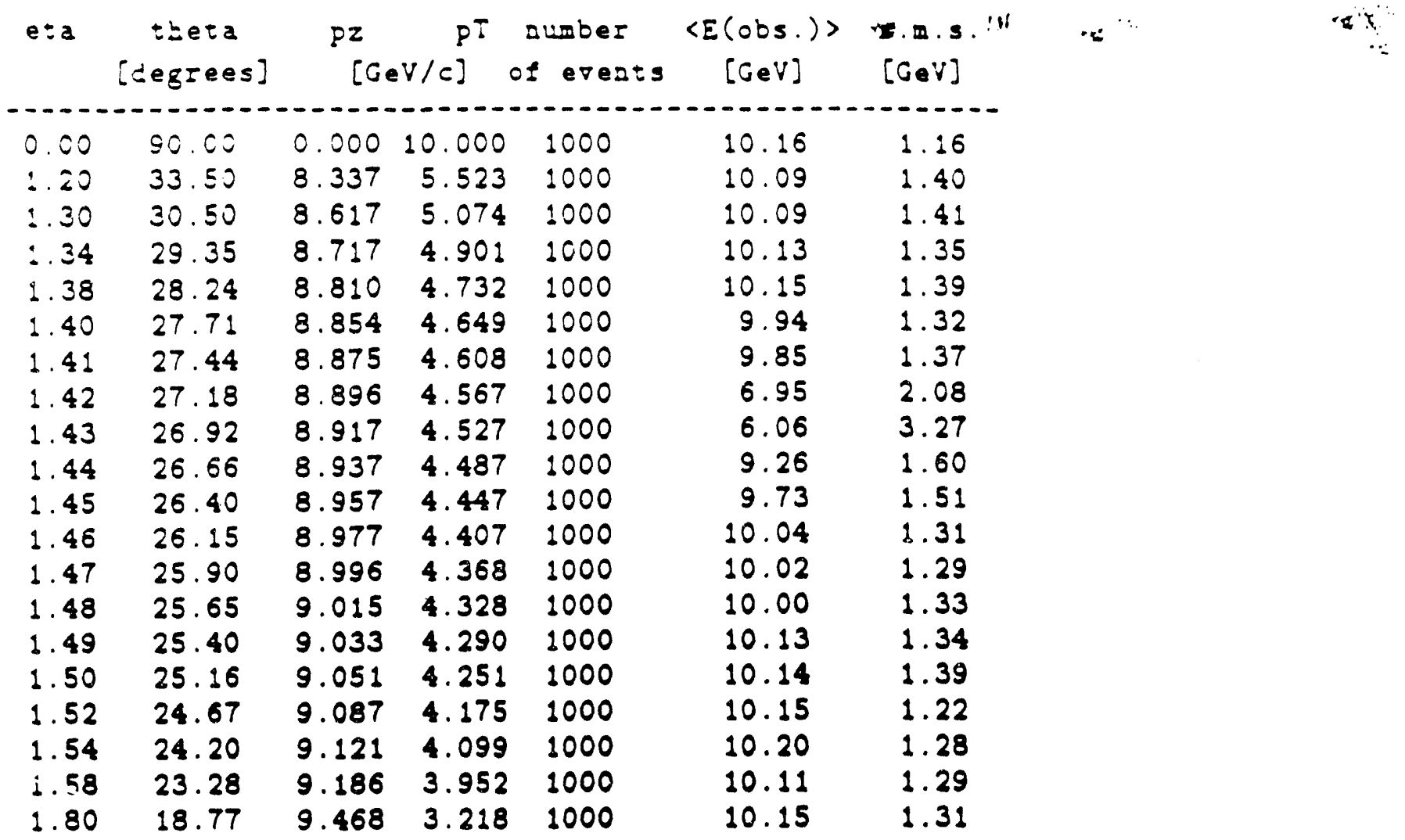




\section{Tabie 2c}

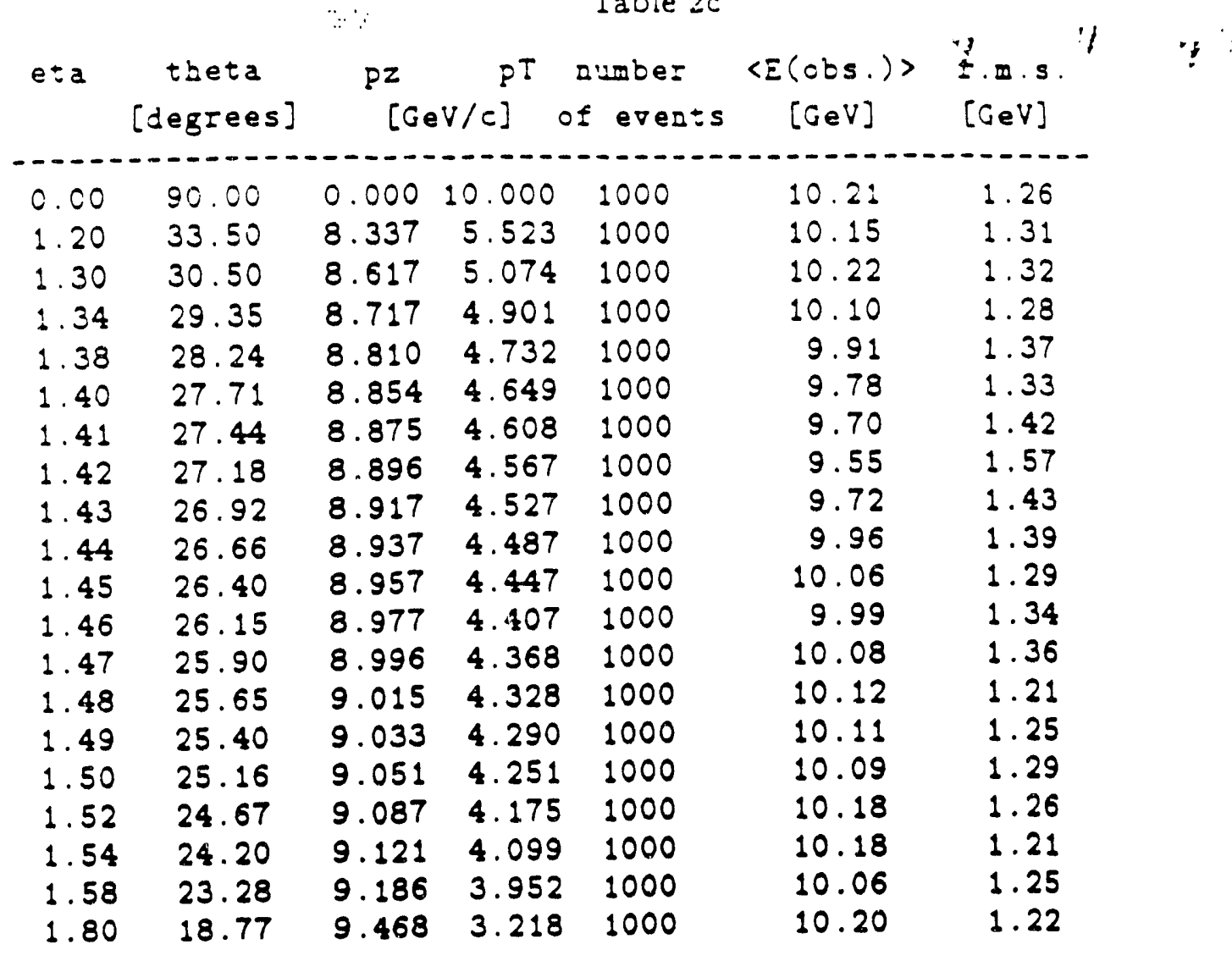




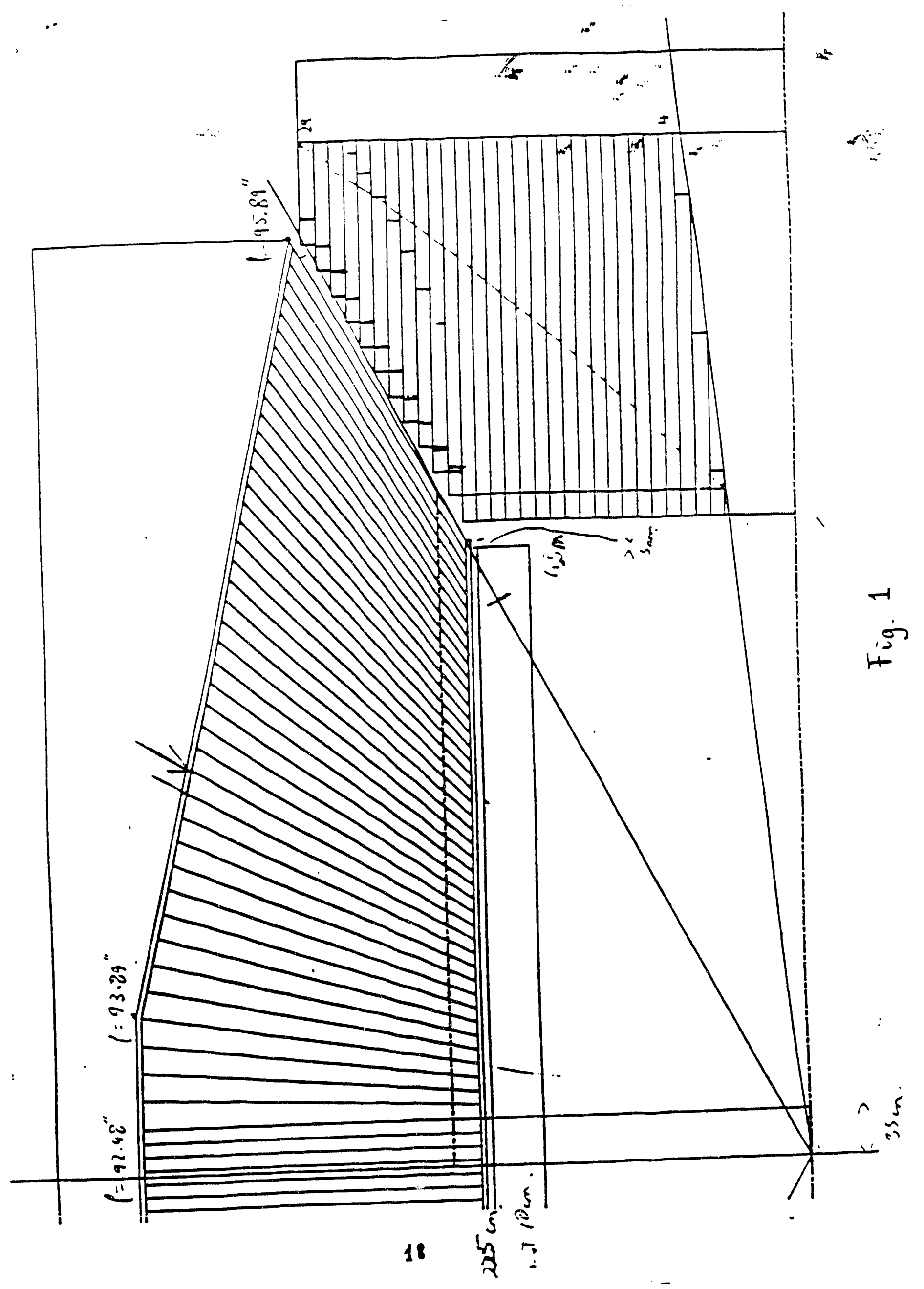




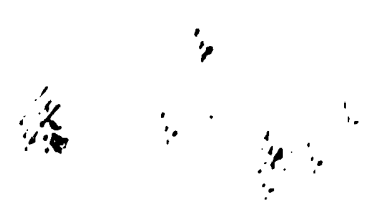

$\because$

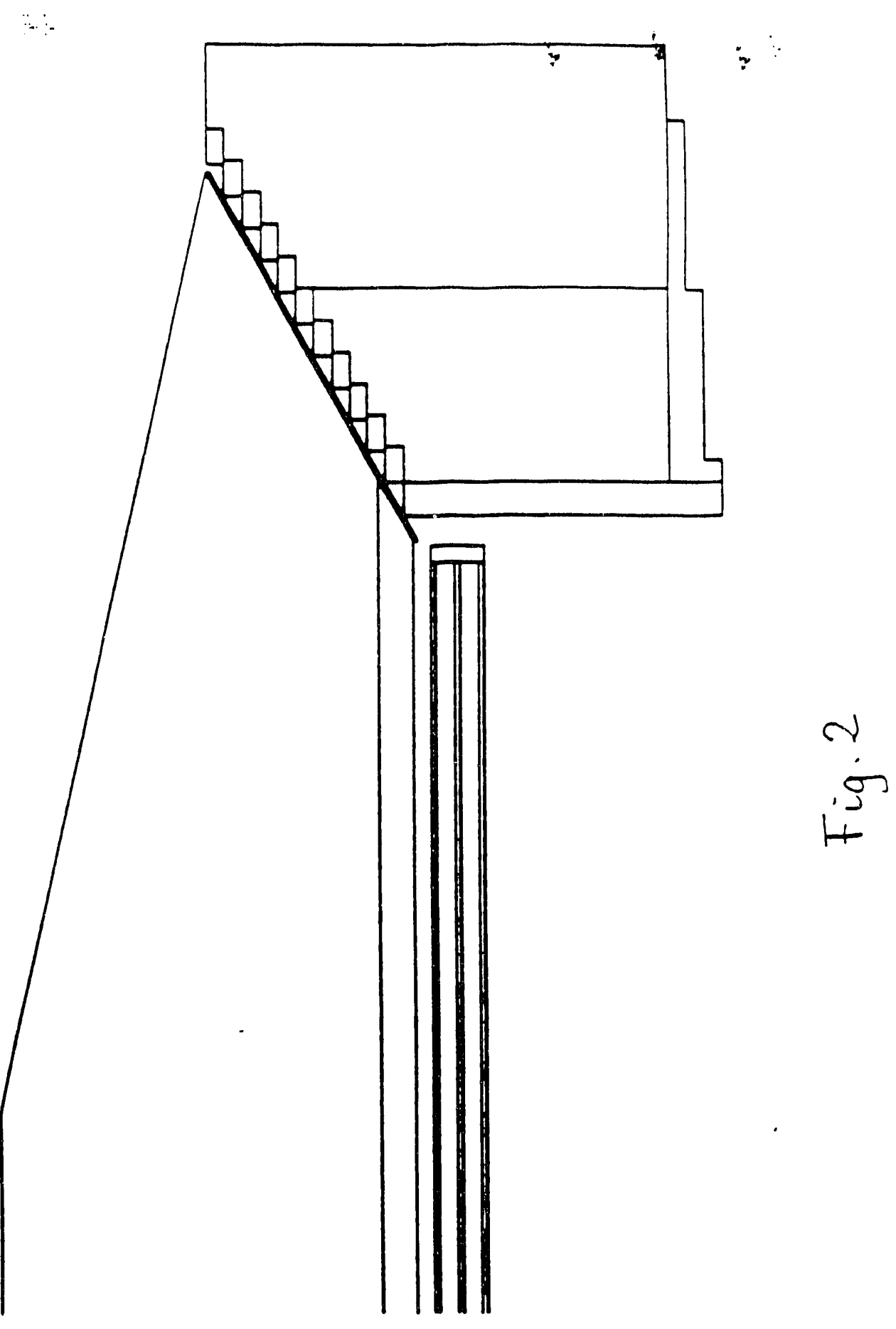



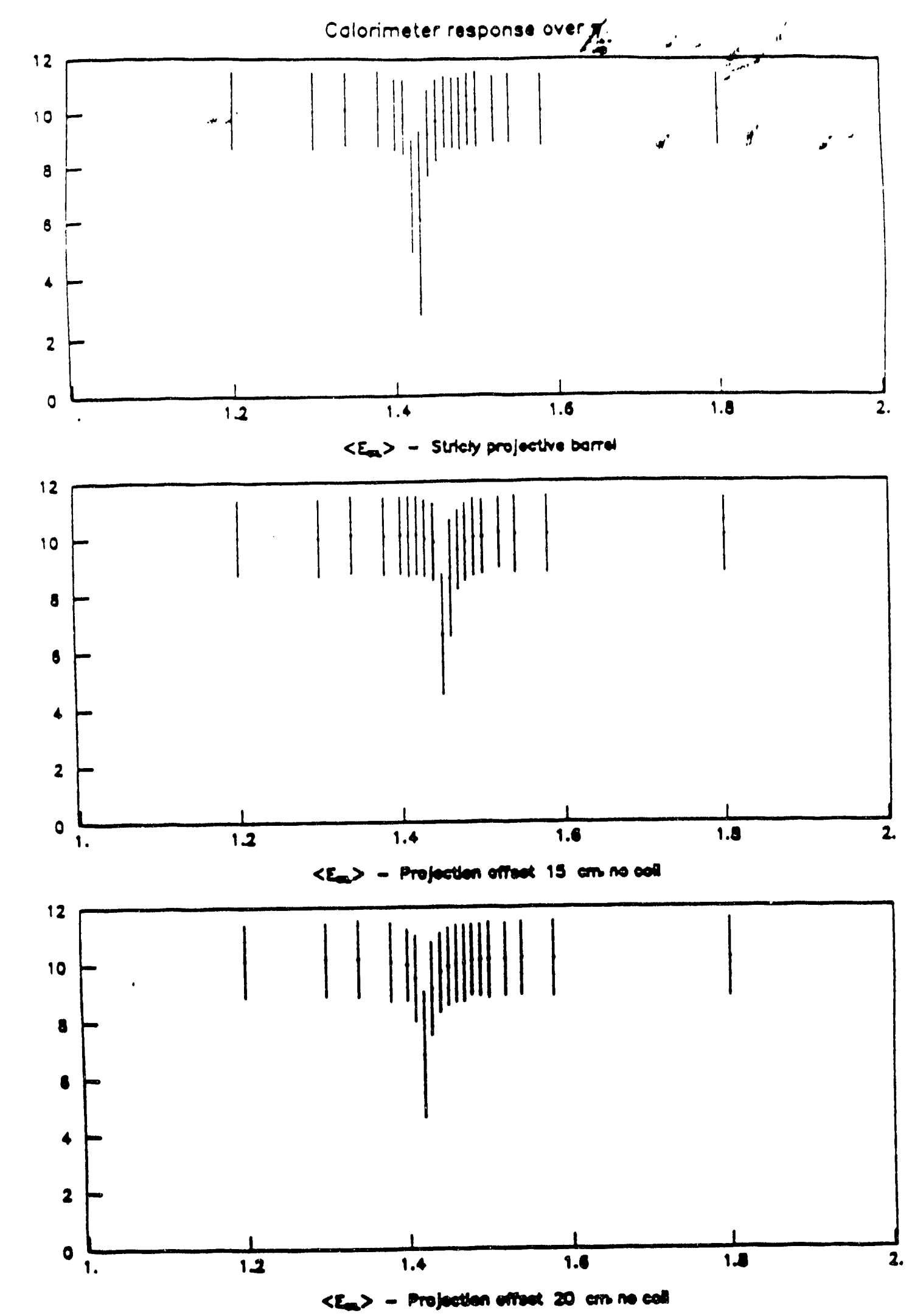

$$
\text { Fig. } 3(1 \text { of } 6)
$$



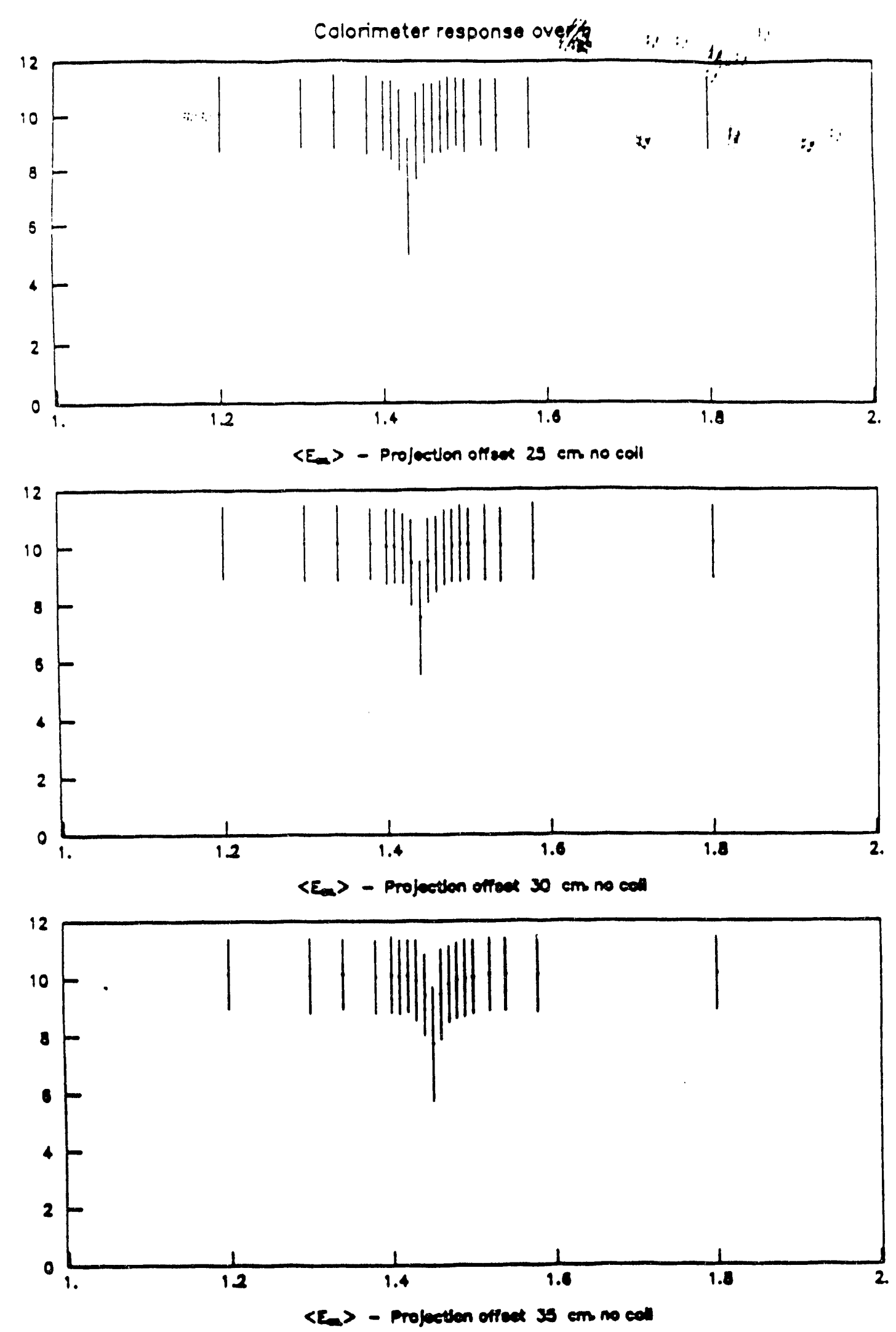

Fig. $3(2$ of 6$)$ 


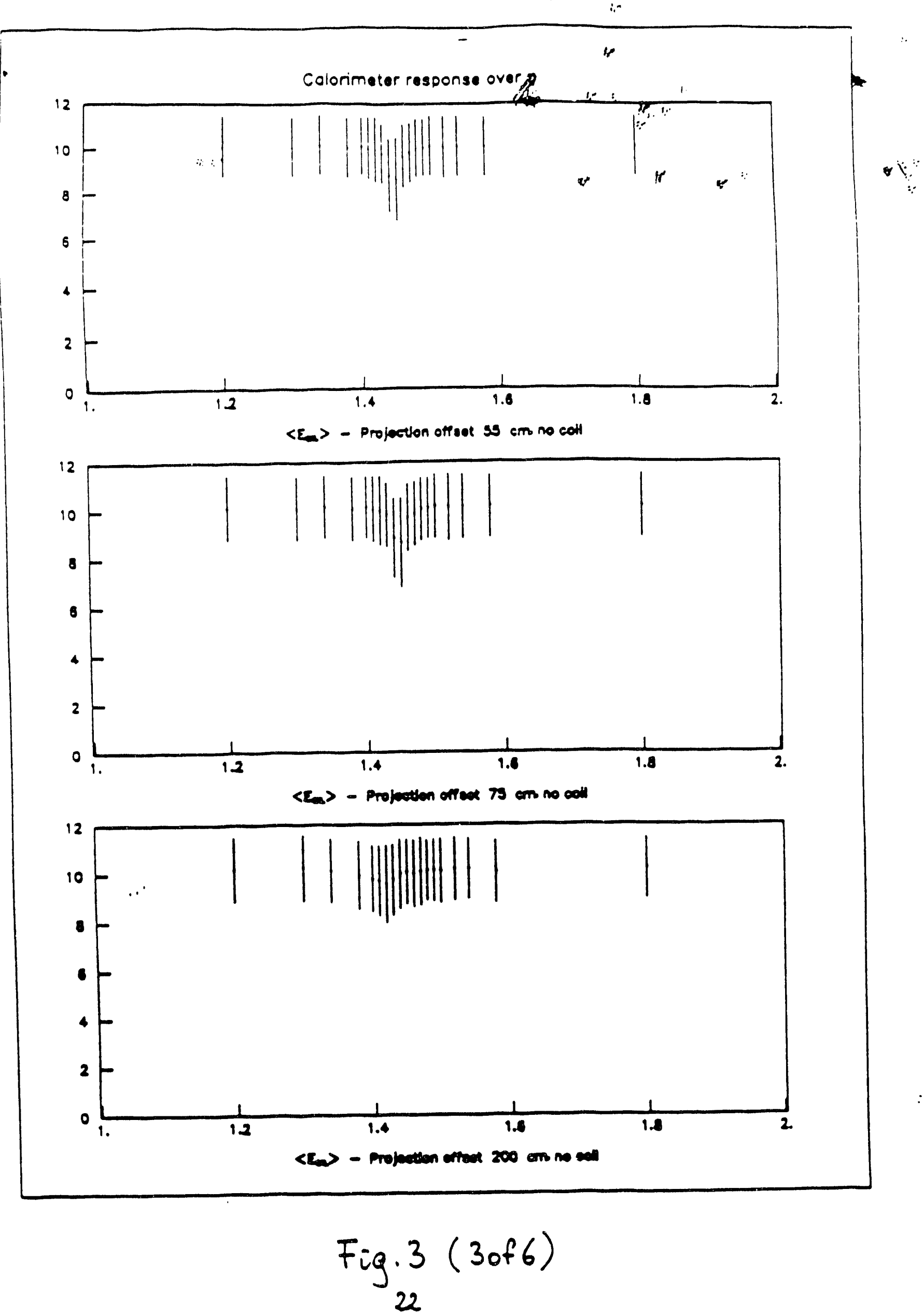



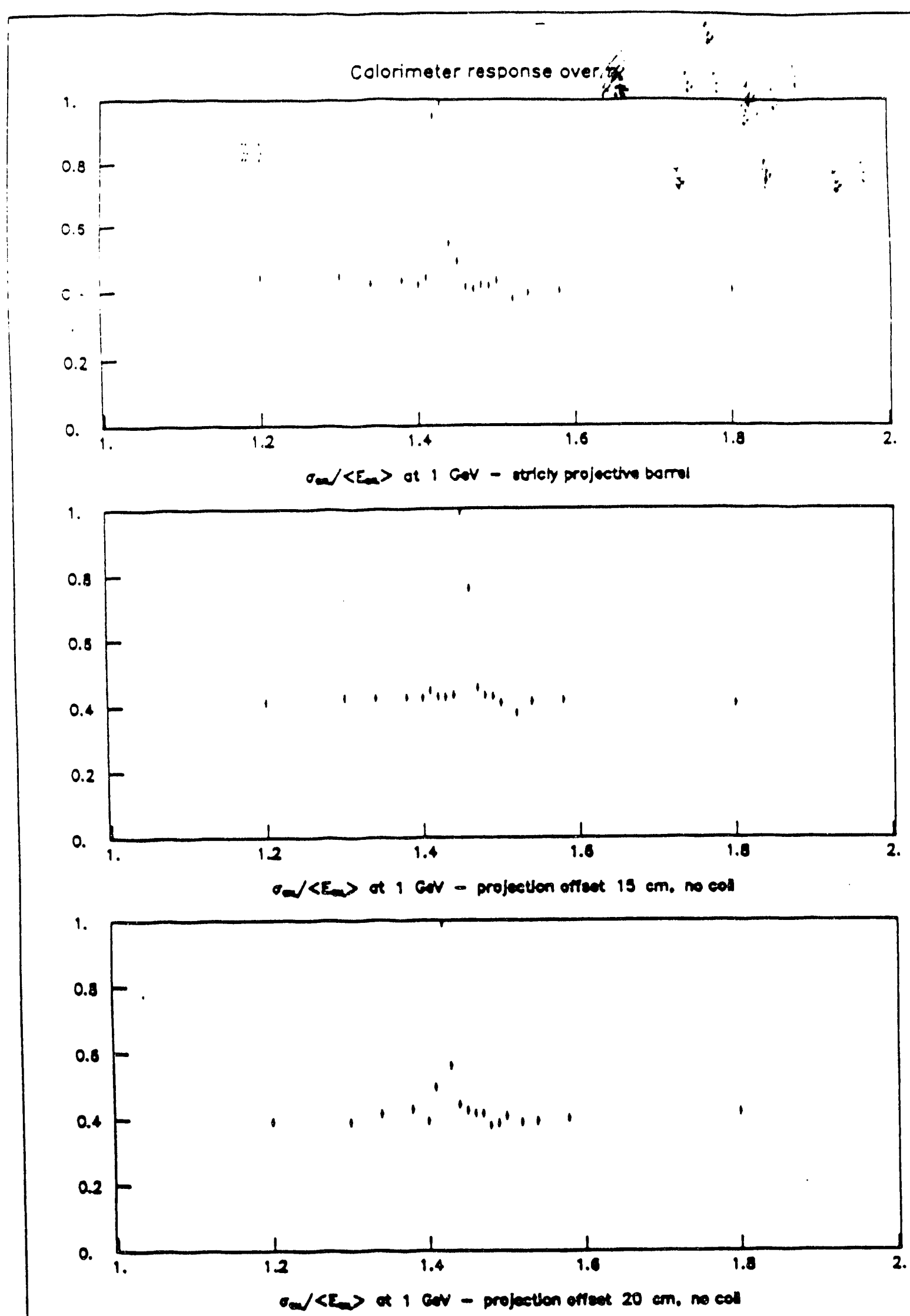

Fig. 3 ( 4 of 6) 
$\therefore$

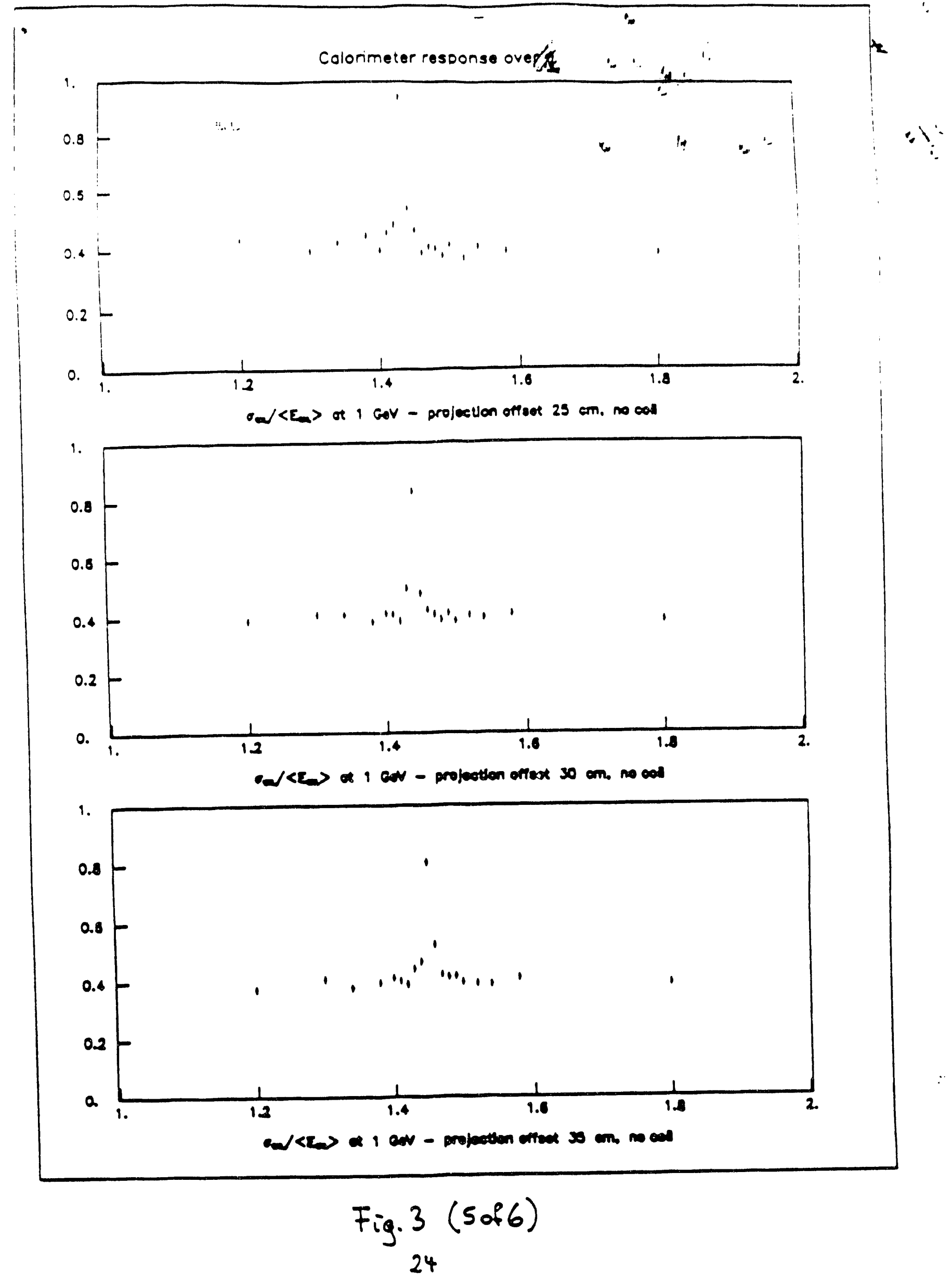




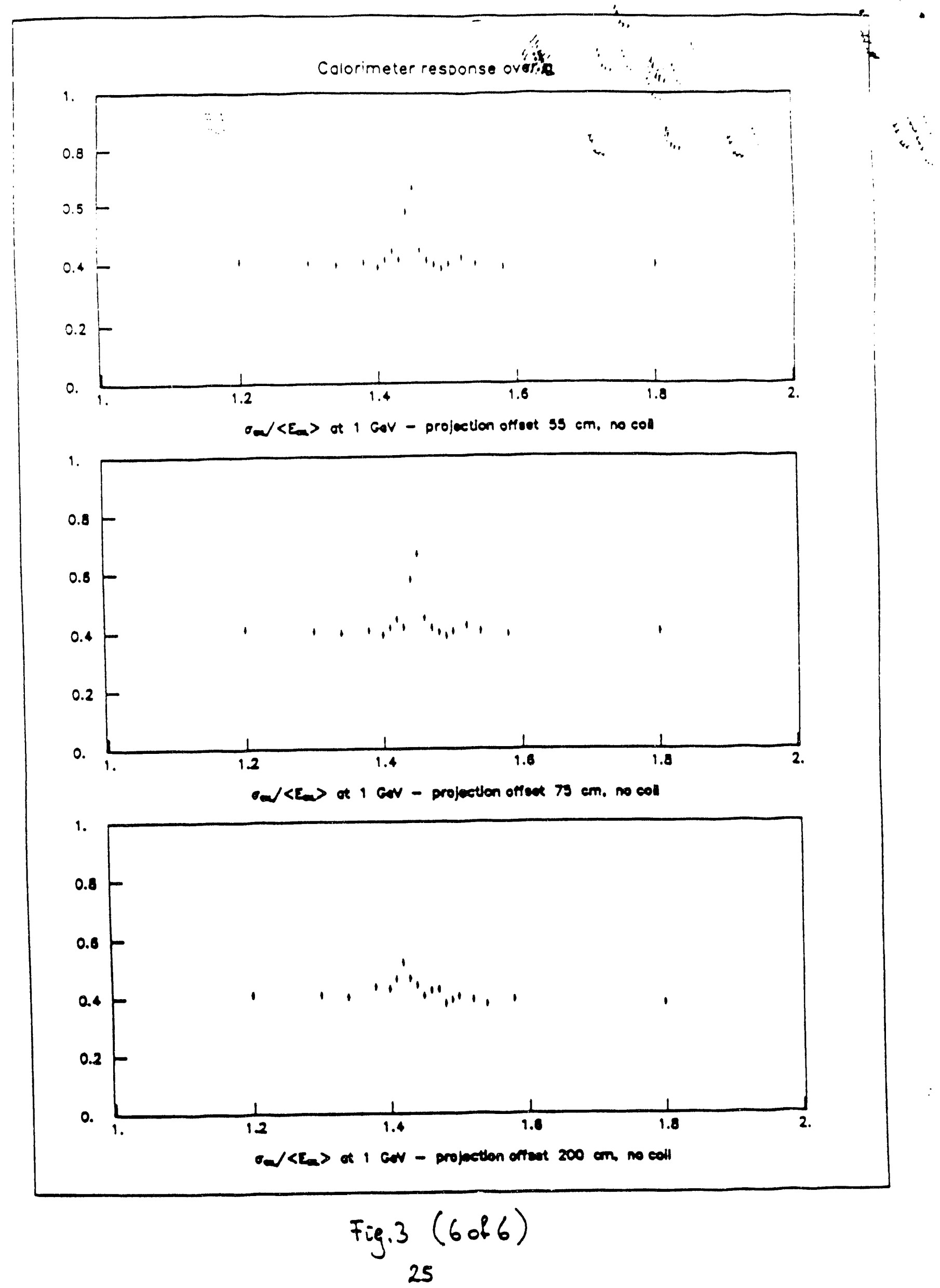




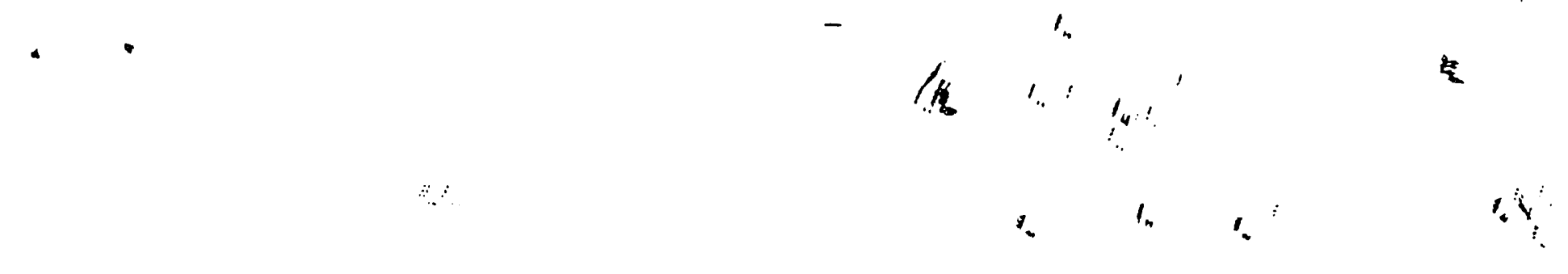

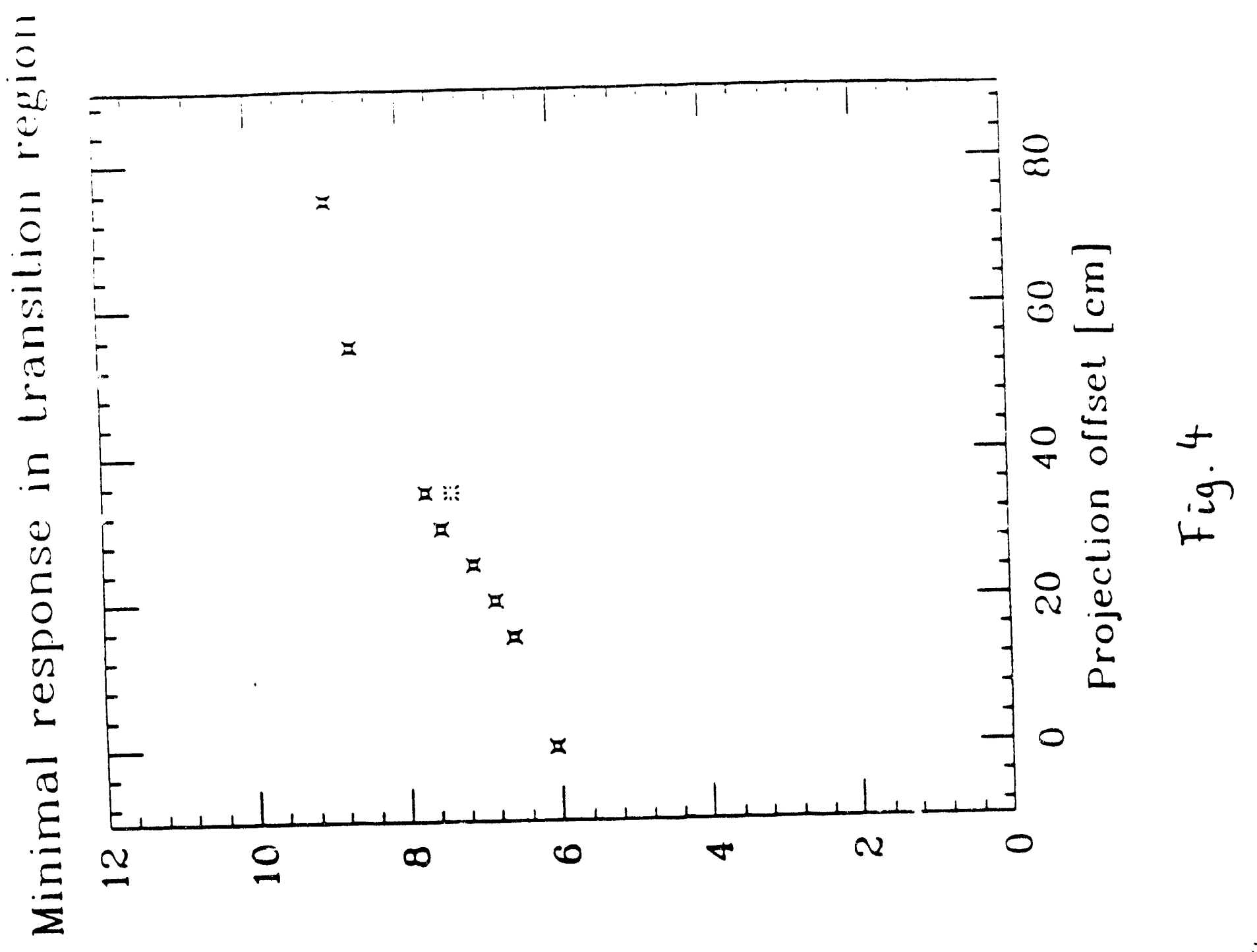

$\left[\Lambda^{\partial \supset}\right]$ asนodsas 7SวMOT 


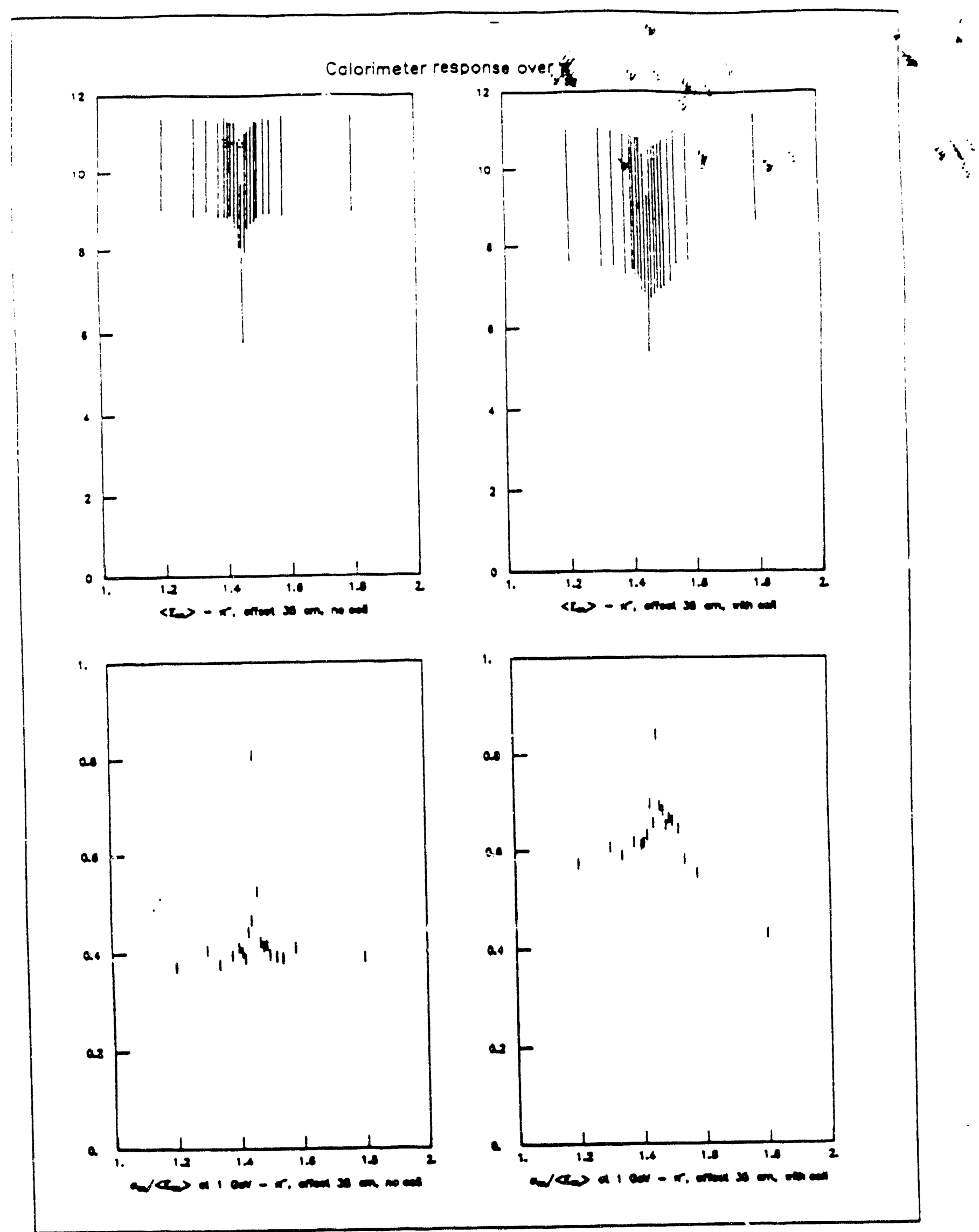

Fiy. 5 


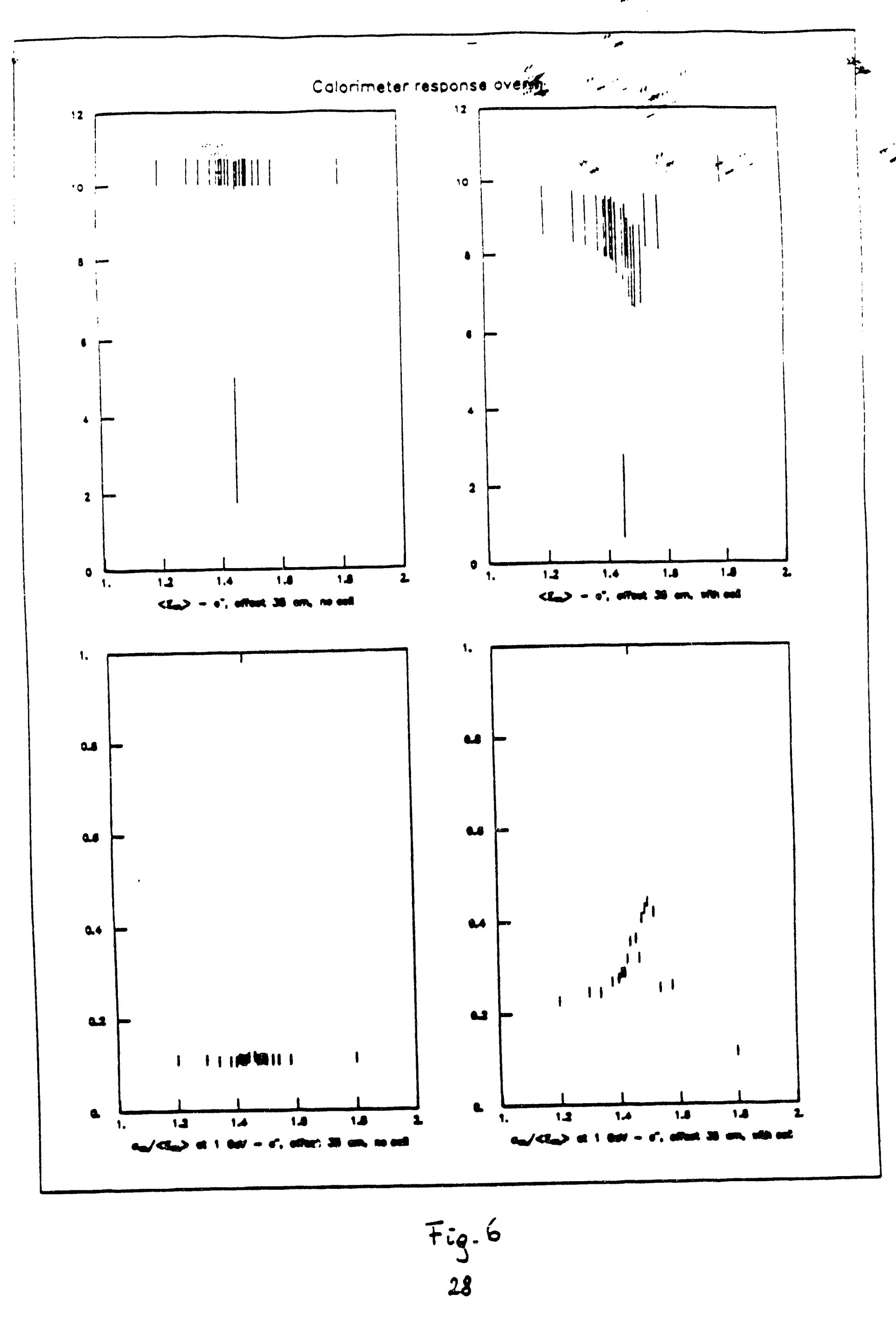




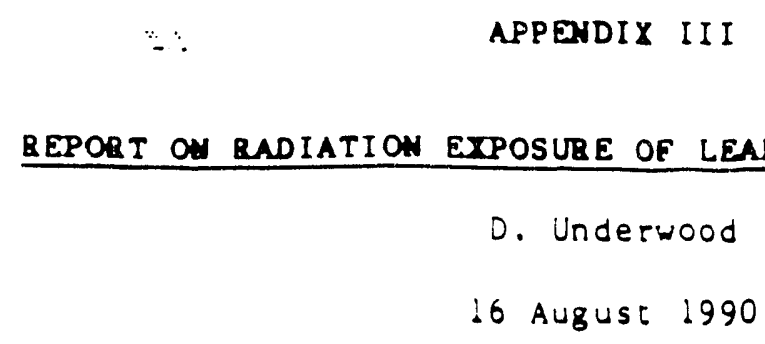

"थ...

\begin{abstract}
A stack of lead and scincillacor was placed in a neutral beam obcained from targeting $800 \mathrm{GeV}$ protons. Small pieces of film containing radiochromic dye were placed adjacent to the layers of scintillator for the purpose of measuring the radiacion dose to the scintillacor. Our motivation was co calibrate the radiation dose obtainable in this maner for fucure tests of scincillacor for SSC experiments. We also observed several ocher radiation effects which should be considered for an SSC calorimeter.

The lead-scincillacor stack was constructed in a simplified manner due to limitations of space and materials. It consisted of CDF scincillator (s mm) at $1 / 2$ and 6 radiation lengths of lead, old scintillator from a protorype (5 mor) at 3 and 4.5 rad. Ien., Y7 waveshifter place $(3 \mathrm{rm})$ at 6 rad. len., and $1 / 2$ rad. Iẹn. of lead behind. Dye filo was placed at .5, 1.5, 3, 4.5, and 6 r.1.
\end{abstract}

The stack was placed at the face of the neutral beam dump of the polarized beas at Fermilab. The neutral bea is frov very forward production and consist asinly of photons and neutrons. The collimator acceptance in the sweeping aegnet is approximety \pm 0.7 ar angle boch horizontally and vertically. Approximataly 1.5 El2 primary beam was cargeted per minute (on a 1... (arget) from July 26 to Auguse 13 ( 18 days with about 302 down time). Approximately 2.5 E 16 protons were targeted. The orter of magnitude neutral 


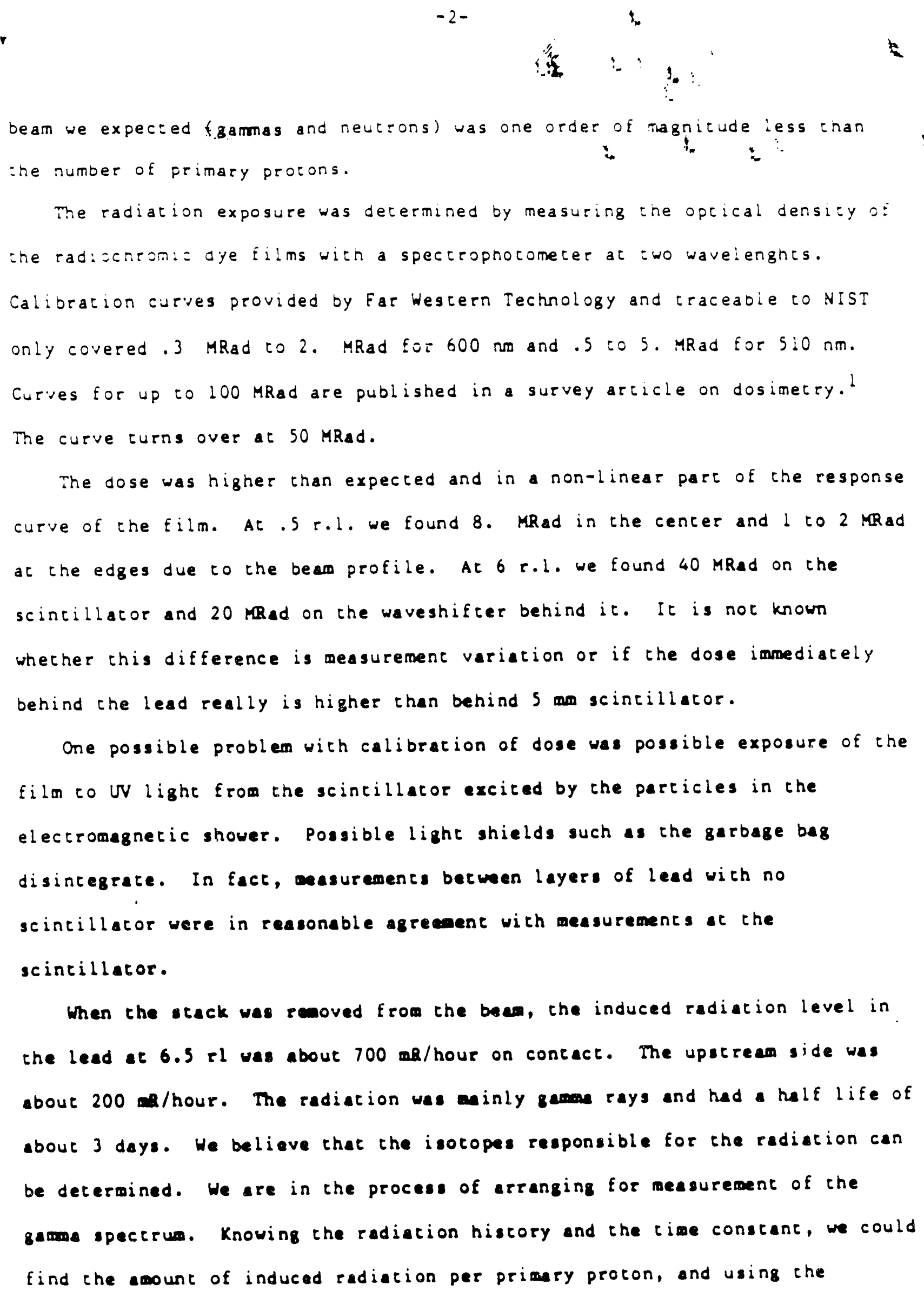

When the sack was removed frow the bean, the induced radiation level in the lead at 6.5 t was about $700 \mathrm{ma} / \mathrm{h}$ our on contact. The upstream gide was about 200 a/hour. The radiation was aninly gama rays and had a half life of about 3 days. He believe that the isotopes responsible for the radiation can be determined. We re in the process of arranging for measurement of the gawo spectrum. Knowing the radiation history and the time constant, we could Find the amount of induced radiation per primery proton, and using the 
radiochromic dye tio measure exposure, we could find induced radiation us.

radiaz:on exposure. The induced activity is probably due to the neucron beam,

but ine hadron to phocon ratio may be cypical for hadronic reacions.

The steady state activicy for constant rate exposure is the dose rate:

rat:o factor " decay time constanc. Crudely, if wat take 40 MR in 28 days and

a time constant of 2.5 days, and .8 Rad activity, the ratio factor is $1.6 \mathrm{E}-7$. When the stack was disassembled after 2.3 days, the scintillator was found to have very lictle induced radiation (less chan. oos of the lead).

When the stack was disassembled, there was radiation induced physical damage to plastic macerials and paper. A thin dark plastic garbage bas disintegrated into small flakes. The paper on the CDF scintillator (the same paper used in the calorimeter) at 6 il which was in contact with the lead turned to powder in the area of the beam, but the paper in contact with the scincillator was not as badly damaged. There was less obvious damage at 0.5 rl. Some white plastic foom used to cushion the older scintillator in compression with the lead disintegraced and stuck to the lead. The glue in stick-on paper labels used ro atcach the dye films became very hard and brictle. The CDF scintillator at 6 el was yellowbrown but the ochers were transparent. The protective paper on the $Y 7$ waveshifter surface had not been removed and becam a white powder which was stuck to the surface.

This experience ay aid in the future to make comparative measurements of the optical properties of damged and undamaged stacks with the configuration of calorimeters. It would also be interesting to relate the dose to the photon and neutron flux if these could be decermined adequately. 
$\sim \cdot r$

$-4-$

4

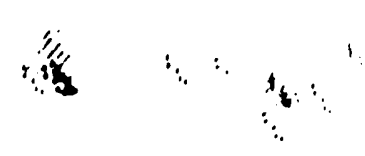

4

A.CKNOWLEDCEMENTS

we thank $j$. Sheppard of ANL HEP for help with the lead, the Fermilab Radiation Safety section for help with activity measurements, M. and J. Peak of ANL BIM for help with the spectrophotometer, and W. McLaughlin of NTIS Eor help in finding calibration informacion.

\section{REFERENCES}

1) MEGAGRAY DOSIMETRY, W.L. Mclaughlin et. al., Radiation Physics and Chemistry, Vol. 22 , p. 33 (1983).

$$
\therefore
$$



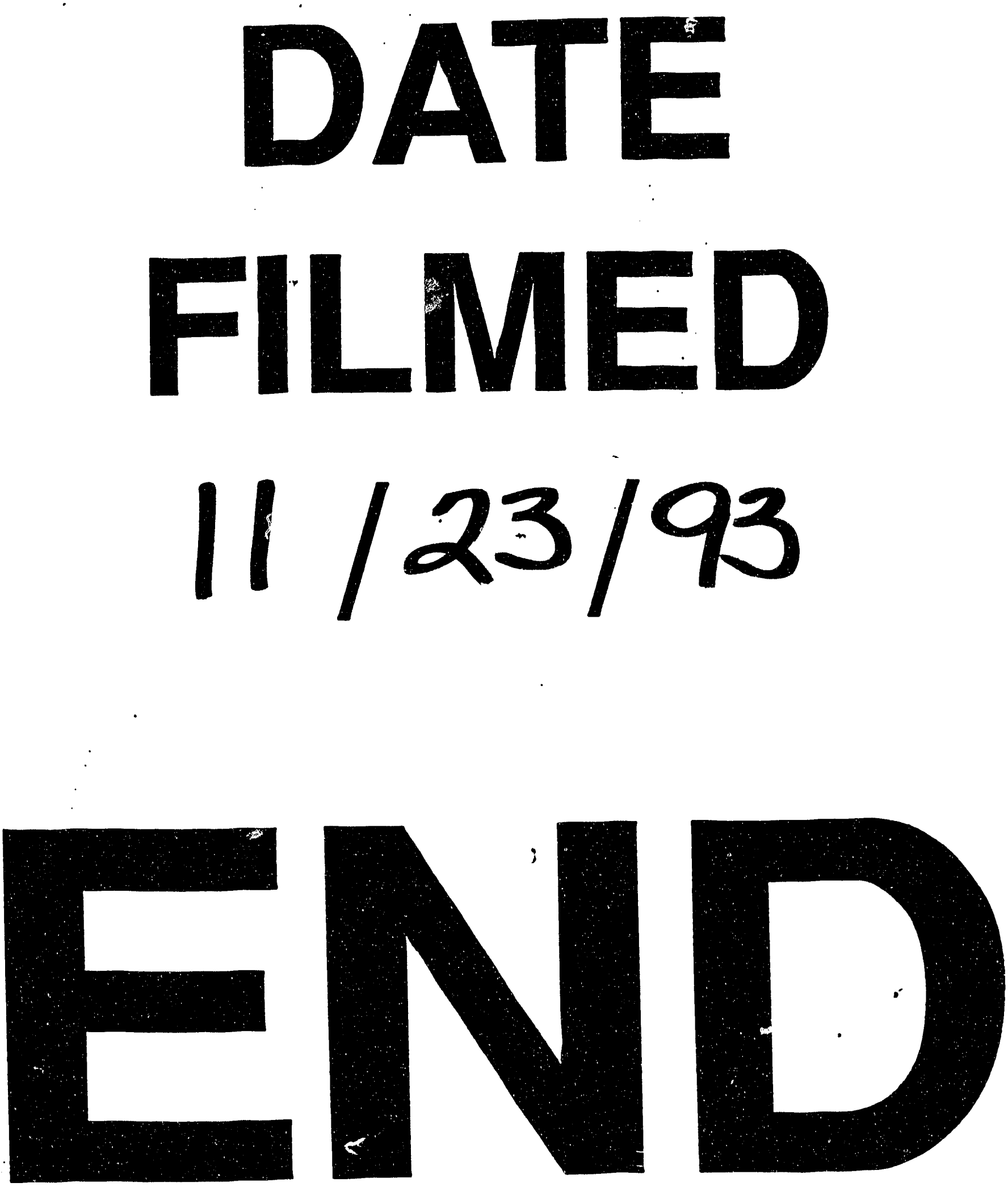
